\title{
MECHANISTIC INVESTIGATION OF 1,5-ANTI STEREOINDUCTION IN ALDOL REACTIONS
}

\author{
by \\ Xuyu Liu

\section{Victoria \\ UNIVERSITY OF WELLINGTON \\ Te Whare Wānanga \\ o te Ūpoko o te Ika a Māui

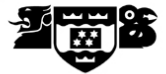

\begin{abstract}
A thesis
submitted to Victoria University of Wellington

in partial fulfilment of the requirements for the degree of

Master of Biomedical Science
\end{abstract}

Victoria University of Wellington

2012 


\section{Abstract}

Peloruside A (+)-1 is a novel secondary metabolite isolated from a New Zealand marine sponge (Mycale hentscheli) by Northcote and West of Victoria University. ${ }^{1}$ Because it has a polyketide backbone, aldol reactions have been widely employed for its total synthesis. Aldol reactions displaying 1,5-anti stereoinduction mediated by the $\mathrm{C}_{15}$ stereocenter (according to peloruside A numbering) have proven useful for the synthesis of the $\mathrm{C}_{11}-\mathrm{C}_{12}$ bond of peloruside $\mathrm{A}$ and analogues. $^{2-5}$

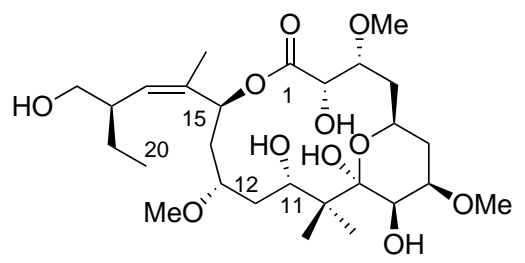

Figure 1: Peloruside A (+)-1

This project is the continuation of Stocker's ${ }^{4,5}$ and Turner's studies ${ }^{2}$ on the excellent stereoinduction of $\mathbf{2}$ in boron-mediated aldol reactions. The relative stereochemistry of the corresponding aldol product is consistence with the expectations of Kishi's ${ }^{13} \mathrm{C}$ database for a 1,5 -anti product. Furthermore, the diphenylsilyl acetal tethered eight-membered ring of $\mathbf{2}$ has proven to be essential for its stereoinduction, while the homoallylic oxygen does not appear to play a significant role.

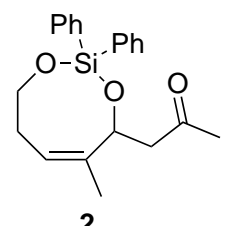

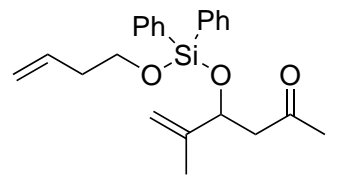

3

Figure 2: $\mathbf{2}$ and $\mathbf{3}$

Although 1,5-anti aldol reactions have been used frequently in the syntheses of polyketidederived natural products, the underlying mechanism for the 1,5-anti-stereoinduction remains 
inconclusive. Three models have been proposed, including Hoberg's $\pi$-stacking model,${ }^{4}$ Goodman's hydrogen-bonding model, ${ }^{6}$ and a modification of Abiko's diborylated model. ${ }^{7}$ The underlying mechanism for the stereoinduction of $\mathbf{2}$ was investigated using variable temperature NMR, 1D NOESY and 1D ROESY experiments. It was found that Hoberg's and Abiko's models are not able to explain the stereoinduction of $\mathbf{2}$ and that Goodman's model used for explaining the transition states of the aldol reaction of $\beta$-trimethylsilyloxy methyl ketones ${ }^{6}$ is also not suitable. A modification of Goodman's model has been proposed to explain the excellent 1,5-anti stereoinduction of $\mathbf{2}$.

While attempts to couple $\mathbf{2}$ and $\mathbf{3}$ to a variety of bulky aldehydes bearing groups with different steric and electronic factors in boron-mediated aldol reactions were unsuccessful, the reaction of 3 with 4-bromobenzaldehyde using $\mathrm{TiCl}_{4}$ and DIPEA afforded an excellent yield (>99\%) of the aldol product. This revealed the six-membered ring in the TS of the boron-mediated aldol reaction is too compact for $\mathbf{2}$ and $\mathbf{3}$. However, it was found that $\mathbf{2}$ is incompatible with $\mathrm{TiCl}_{4}$.

Key questions regarding the 1,5-anti-stereoinduction of $\mathbf{2}$ have been answered and a modified procedure for the NMR investigation of an aldol reaction is described in this thesis. 


\section{Acknowledgements}

Like any other scientific project, I would not have achieved the work in this thesis without my supporting team. First I must thank my supervisors. Thank you for believing in me, and thank you for your patient, plentiful support and knowledge. Paul - your honesty and diverse imagination is incredible. You always have time for me, even if you have to juggle so much at the same time. Thank you for your understanding and diversely extending my ideas and for help with computational modelling. Joanne - there are very few chemistry questions you cannot answer! Your enthusiasm for chemistry encourages me all the time. Thanks for your patience and help in meetings and in the lab.

Thank you to all the lab members in our team, Russell (past lab-babysitter), Mark (strict lab-babysitter now), Hemi (for your help and discussion in chemistry), Febly (for your guidance in experiments), Peter (for your interesting ideas and sharing jokes when we were working late in the lab), Kalpani, Claire, Sam, Claudia, Thomas, Dylan and Scott.

I must also thank all of the technical and admin staff in SCPS, especially Dr. Ryan and Ian for your help of NMR experiments. And Dr. Keyzers, thank you for your guidance in HP20 column use and your useful information about NMR structural determination. And thanks to all the academic staff - especially Prof. Spencer, Prof. Halton and Assoc. Prof. Northcote.

I am not a very lively person but I am lucky enough to have a few friends in China and New Zealand who always support and organize entertainment for me! Thanks for Manyu, Xiaoting, Chen, Jiyun, Jimmy, Jianing and all other lovely friends.

Finally, I have to thank my Mum for cooking delicious meals for me and for always having time to listen to my imagination of science, even if science is not your favorable subject! I also like to thank my Dad for your hard work in China to support our family. And thanks for my lovely cousins in China and New Zealand for your support. 


\section{Table of Contents}

$\begin{array}{ll}\text { Abstract } & \text { ii }\end{array}$

$\begin{array}{ll}\text { Acknowledgements } & \text { iv }\end{array}$

$\begin{array}{ll}\text { List of Figures } & \text { vii }\end{array}$

$\begin{array}{ll}\text { List of Schemes } & \text { ix }\end{array}$

List of Tables $\quad$ xi

$\begin{array}{ll}\text { Glossary } & \text { xii }\end{array}$

1 Background and Introduction $\quad 1$

1.1 Polyketide Biosynthesis .......................... 1

1.1.1 Classification of Polyketide Synthases (PKSs) . . . . . . . . . . . 2

1.1.2 Biosynthesis of DEBS . . . . . . . . . . . . . . . . 2

1.2 History and Biochemistry of Peloruside A . . . . . . . . . . . . . . . . 3

1.3 Asymmetric Aldol Reactions . . . . . . . . . . . . . . . . . . . . 4

1.3.1 1,2-Asymmetric Induction in Chiral Aldehydes . . . . . . . . . . . 6

1.3.2 1,3-Anti Induction in Chiral Aldehydes . . . . . . . . . . . . . . 10

1.3.3 1,2- and 1,3-Merged Asymmetric Induction in Chiral Aldehydes . . . . . . 13

1.3.4 1,4-Syn and Anti Induction in Aldol Coupling of (Z)-Enolates . . . . . . 15

1.4 "Propionate-Type" and "Acetate-Type" Aldol Reactions and 1,5-Anti Stereoin-

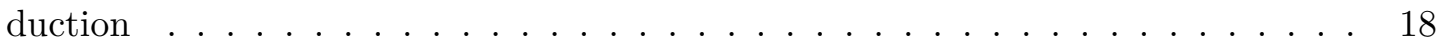

1.4.1 Transition States of Acetate- and Propionate-Type Aldol Reactions . . . . 18

$1.4 .2 \quad 1,5$-Anti Induction in Aldol Reaction . . . . . . . . . . . . . . . 22

2 Objectives of the Research $\quad 34$

3 Results and Discussion $\quad 35$

3.1 Synthesis of Dialkylboron Triflate and Chloride Reagents . . . . . . . . . . . . . 35

3.2 Syntheses of $\beta$-Hydroxy Methyl Ketones . . . . . . . . . . . . . . . . . . . 37

3.2.1 Paterson's Asymmetric Aldol Reaction and Synthesis of $(S)-18$. . . . . 37

3.2.2 List's Asymmetric Aldol Reaction and Synthesis of $(R)-\mathbf{1 8}$. . . . . . . 39 
3.2.3 Synthesis of Racemic $18 \ldots \ldots$. . . . . . . . . . . . . . . . 40

3.3 Syntheses of Protected $\beta$-Oxygenated Methyl Ketones . . . . . . . . . . . . . 41

3.3.1 Preparation of Various Acyclic Protected $\beta$-Oxygenated Methyl Ketones . 41

3.3.2 Preparation of Cyclic $\beta$-Oxygenated Methyl Ketone 2 . . . . . . . . . . . 44

3.4 Aldol Reactions of Various $\beta$-Oxygenated Ketones . . . . . . . . . . . . . . . 48

3.4.1 Determination of the Relative Stereochemistry of 28a . . . . . . . . . 48

3.4.2 Aldol Reactions of Protected $\beta$-Oxygenated Methyl Ketones . . . . . . . . 54

3.4.3 Aldol Reactions of 3 Mediated by Lithium and Titanium . . . . . . . . . 59

3.4.4 Questions About Goodman's model . . . . . . . . . . . . . . . 62

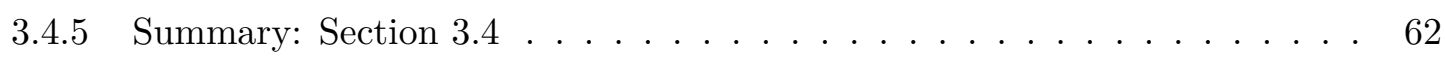

3.5 Conformational Studies Using Various NMR Experiments . . . . . . . . . . . . 64

3.5.1 Conformational Studies of 2 and $3 \ldots \ldots \ldots \ldots$. . . . . . . . 64

3.5.2 NMR Study of the Enolization and the Aldol Reaction of 2-Hexanone . . 68

3.5.3 NMR Studies of Aldol Reactions of 2 and 3 . . . . . . . . . . . 75

3.5.4 Proposed Mechanism for 1,5-anti Stereoinduction of 4a in Aldol Reaction 90

3.5.5 Summary: Section $3.5 \ldots \ldots \ldots \ldots$. . . . . . . . . . . . 93

3.6 Model Studies of $\mathrm{C}_{11}-\mathrm{C}_{12}$ Bond Connection in Syntheses of Peloruside A Analogues 94

4 Future Work $\quad 96$

4.1 Future Work for Synthesis of $2 \ldots \ldots \ldots$. . . . . . . . . . 96

4.2 Future Work for Investigating the Underlying Stereoinducing Mechanism of 2 . 98

5 Experimental Procedures $\quad 100$

5.1 General . . . . . . . . . . . . . . . . . . . . 100

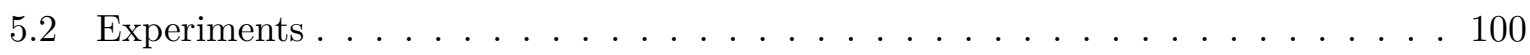

$\begin{array}{ll}\text { References } & 113\end{array}$ 


\section{List of Figures}

1 Peloruside $\mathrm{A}(+)-1 \ldots \ldots \ldots \ldots \ldots \ldots$ ii

$2 \quad 2$ and $3 \ldots \ldots \ldots \ldots \ldots \ldots$ ii

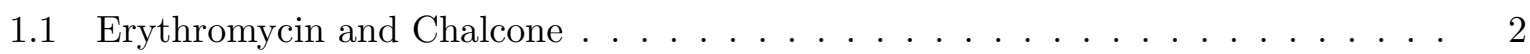

1.2 Biosynthesis of 6-Deoxyerythronolide B (DEB) by Non-Iterative Type I Polyketide Synthase . . . . . . . . . . . . . . . . . . 3

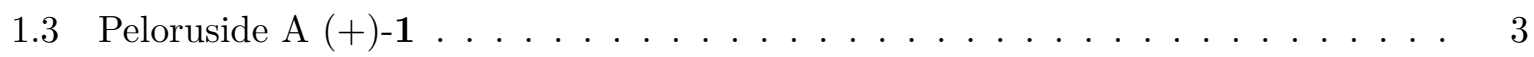

1.4 Possible Stereoinducing Chiral Centers in the Product of a Substrate-Directed Aldol Reaction . . . . . . . . . . . . . . . . . . 6

1.5 Goodman's Calculated Transition States of Butan-2-one Derived Enolates and Acetaldehydes (Relative Energies in kcal mol ${ }^{-1}$, Reproduced from Goodman's Paper $^{17}$ by Permission of The Royal Society of Chemistry) . . . . . . . . . . . 22

1.6 Goodman's Calculated Transition States of Acetone Derived Enolates and Acetaldehydes (Relative Energies in kcal mol ${ }^{-1}$, Reproduced from Goodman's Paper $^{17}$ by Permission of The Royal Society of Chemistry) . . . . . . . . . . . . . 23

1.7 Hoberg's Model for 1,5-Anti-Induction in Aldol Reaction ${ }^{4,6} \ldots \ldots$. . . . . . . 25

1.8 Goodman's Model for 1,5-anti-Induction in Aldol Reaction (Reproduced from Goodman's Paper ${ }^{6}$ by Permission of The American Chemical Society) . . . . . . 27

1.9 Generic Goodman's Transition States . . . . . . . . . . . . . . . . 28

1.10 Goodman's Model for $\beta$-siloxy Directing 1,5-Anti-Induction (Reproduced from Goodman's Paper ${ }^{6}$ by Permission of The American Chemical Society) . . . . . . 29

3.1 Five Methyl Ketones used in the Mechanistic Investigation of 1,5-anti-Aldol Re-

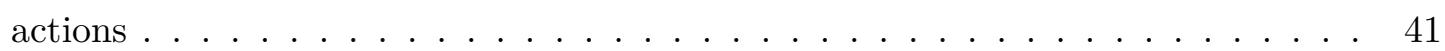

3.2 Grubbs 2nd Generation Catalyst (25) . . . . . . . . . . . . . . . 45

3.3 Kishi's ${ }^{13} \mathrm{C}$ NMR Database of $1,3,5$-Triols ${ }^{53} \ldots \ldots \ldots \ldots$

3.4 Key ${ }^{13} \mathrm{C}$ and ${ }^{1} \mathrm{H}$ NMR Spectral Data and Key HMBC Correlations of $29 \ldots 3$

3.5 Relative Stereochemistry Determination of 33a Using Polarimeter . . . . . . . 57

3.6 Full nOe Correlations of $2 \ldots \ldots \ldots \ldots \ldots$

3.7 Possible Conformers of the Eight-Membered Ring of $2 \ldots \ldots$. . . . . . . 66

3.8 Conformation of $\mathrm{C}_{3}-\mathrm{C}_{4}$ Bond of $2 \ldots \ldots \ldots \ldots \ldots$

3.9 Detailed nOe Correlations of $\mathbf{3} \ldots \ldots \ldots \ldots \ldots$

3.10 Crude ${ }^{1} \mathrm{H}$ NMR Spectrum of 41 (Generated at $-20{ }^{\circ} \mathrm{C}$ ) . . . . . . . . . 72 
3.11 Selective ${ }^{1} \mathrm{H}$ NMR Spectral Data of 2 -Hexanone and $41 \ldots \ldots$. . . . . . . 73

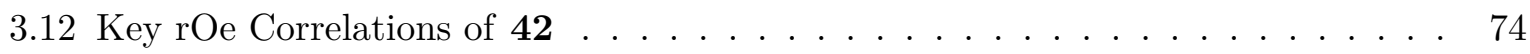

3.13 Possible Conformers for $42 \ldots \ldots \ldots \ldots$. . . . . . . . . . . . 74

3.14 Key ${ }^{1} \mathrm{H}$ Spectral Data of $48 \ldots \ldots \ldots \ldots \ldots \ldots$. . . . . . . . . . . . . . . . . . . . . . 77

3.15 Crude ${ }^{1} \mathrm{H}$ NMR Spectrum of 48 (Generated at $-20{ }^{\circ} \mathrm{C}$ ) . . . . . . . . . . 79

3.16 Downfield-Shifting Effects from the $\beta$-Diphenylsiloxy Groups $\left({ }^{1} \mathrm{H}\right.$ NMR Spectral

Data Obtained in $\left.\mathrm{CDCl}_{3}\right) \ldots \ldots \ldots \ldots$. . . . . . . . . . . . 80

3.17 Stacked ${ }^{1} \mathrm{H}$ NMR Spectra of 41 (Blue) and 48 (Red) . . . . . . . . . . . . 81

3.18 Key rOe Correlation of $\mathbf{4 3 b}$ and Expectated rOe Correlations in Different Con-

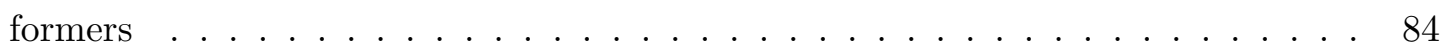

3.19 Key rOe Correlations of $44 \ldots \ldots \ldots \ldots \ldots$

3.20 Expected Stable Conformers of $44 \mathbf{a} \ldots \ldots \ldots \ldots$. . . . . . . . . 88

3.21 Unstable Conformers of $44 \mathrm{a} \ldots \ldots \ldots \ldots$. . . . . . . . . . . . 89

3.22 Modified Goodman's Syn-Boat Conformers for TS . . . . . . . . . . . . . . . . . . 91

3.23 Modified Goodman's Anti-Boat Conformers for TS . . . . . . . . . . . . . . . . . 92

3.24 Aldehydes Described in this Section . . . . . . . . . . . . . . . 94 


\section{List of Schemes}

1.1 Transition States and Corresponding Aldol Products of $(E)$ - and $(Z)$-Enolates ${ }^{17} 5$

1.2 Cram and Felkin Models ${ }^{18,19} \ldots \ldots \ldots \ldots \ldots$

1.3 Improved Felkin Model and Polar Felkin-Anh Model $^{20} \ldots \ldots$. . . . . . . . 7

1.4 Evans-Cornforth Model for Explaining 1,2-anti-Aldol Chemistry ${ }^{22} \ldots \ldots$. . . 8

1.5 Felkin and Cram-chelation Products Generated from Different Reagents ${ }^{23} \ldots$. . 9

$1.61,3$-Anti Stereoinduction of a Protected $\beta$-Hydroxy Aldehyde in Nucleophilic Addition to a Carbonyl . . . . . . . . . . . . . . . . . . . . 11

1.7 Boron Trifloride-Mediated 1,3-Anti Allylation and Aldol Reactions of Aldehydes via Acyclic Transition States ${ }^{29,30} \ldots \ldots$. . . . . . . . . . . . . 12

1.8 Merged 1,2- and 1,3-Asymmetric Induction in Mukaiyama and Lithium-enolate Aldol Reactions According to Evans et al. ${ }^{15} \ldots$. . . . . . . . . . . . . . . 14

1.9 Evans' Models for Explaining 1,2- and 1,3-Asymmetric Induction of "Mismatched" Aldehyde in Mukaiyama Aldol Reaction . . . . . . . . . . . . . . . . . . . . 15

$1.101,4-$ Syn and Anti-Induction ${ }^{31} \ldots \ldots \ldots \ldots \ldots$. . . . . . . . . . . 17

1.11 Asymmetric Enolizations and Aldol Reactions with (-)-(Ipc) ${ }_{2}$ BOTf and (-)-(Ipc) ${ }_{2} \mathrm{BCl}$ According to Paterson ${ }^{32} \ldots \ldots \ldots \ldots$. . . . . . . . . . . . . . 19

1.12 Paterson's Models for Diisopinocampheyl Boron Mediated Asymmetric Aldol Re-

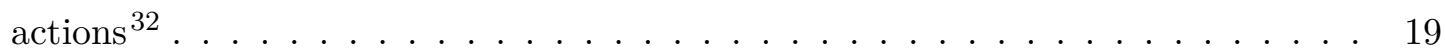

1.13 Menthol-derived Chiral Boron Chloride Directing Asymmetric Aldol Reactions of Methyl Ketone and $\alpha$-Amino Aldehyde ${ }^{14,34} \ldots \ldots \ldots \ldots$

1.14 1,5-Anti Induction in Aldol Reaction with Different Protecting Groups ${ }^{37,38}$. . . 25

1.15 1,5-Anti-Stereoinduction from Different Silyl Protected Methyl Ketones ${ }^{2,4,5}$. . 26

1.16 Bis-Aldol Reactions of $\mathbf{7}$ and Evidence for the Formation of Doubly Borylated Enolate $9 \ldots \ldots \ldots \ldots \ldots \ldots$

1.17 Investigation of $(E)$ - and $(Z)-\mathbf{1 0}$ and the Corresponding Aldol Products with Variable Temperature ${ }^{1} \mathrm{H}$ NMR Experiments ${ }^{7} \ldots \ldots \ldots \ldots$. . . . . . . . . . 32

1.18 Proposed Mechanism for Bis-Aldol Reaction of Acetate Ester Proposed by Abiko et al. ${ }^{7} \ldots \ldots \ldots \ldots \ldots \ldots \ldots \ldots \ldots$

3.1 Syntheses of Boron Triflate and Chloride Reagents using Paterson's, Abiko's and Reider's Methods ${ }^{32} \ldots \ldots \ldots \ldots$. . . . . . . . . . . . . 36

3.2 Acetone Aldol Reactions Employed in the Syntheses of (S)-, (R) and (rac)-18 $\mathbf{1}^{2,32,43} 37$ 
3.3 Possible Decomposition Pathways by E1cB Elimination ${ }^{44} \ldots \ldots \ldots$. . . . . . 38

3.4 List's Enamine Mechanism of $L$-Proline Catalyzed Asymmetric Aldol Reaction ${ }^{43} 39$

3.5 Synthesis of $(r a c)-6 \mathbf{b}$ reported by Turner ${ }^{2} \ldots \ldots \ldots \ldots \ldots$. . . . . . . 42

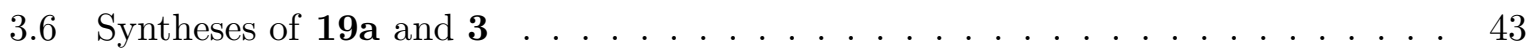

3.7 Syntheses of 5 and $20^{2,44} \ldots \ldots \ldots \ldots \ldots \ldots \ldots \ldots$

3.8 Ring Closing Metathesis Reactions $4,48 \ldots \ldots \ldots \ldots \ldots$. . . . . . . . 46

3.9 Aldol Reaction of $\mathbf{2}$ with $\mathbf{2 7 a} \ldots \ldots \ldots \ldots$. . . . . . . . . . . . 48

3.10 Evans-Chapman-Carreira Reduction of $\mathbf{2 8 a}^{6} \ldots \ldots \ldots \ldots$. . . . . . . 51

3.11 Modified Goodman's Boat TS for Evans-Chapman-Carreira Reduction of $\mathbf{2 8} \mathbf{a}^{6}$. 52

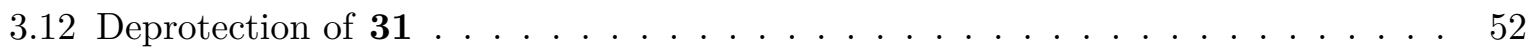

3.13 Tentative Deduction of the Relative Stereochemistry of 28a and $31 \ldots \ldots 4$

3.14 NMR References of Anti-Diols . . . . . . . . . . . . . . . 54

3.15 Aldol Reaction of $\mathbf{2 0}$ with $\mathbf{2 7} \mathbf{b} \ldots \ldots \ldots \ldots \ldots \ldots$

3.16 A Proposed Two-Step Chemical Modification to Determine the Relative Stereo-

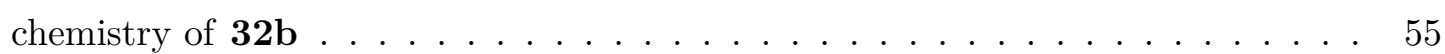

3.17 Relative Stereochemistry Determination of 1,3-Diols Using 4-Methoxybenzylidene

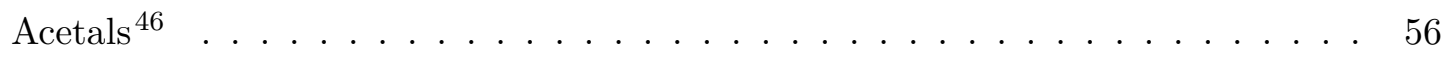

3.18 Aldol Reactions of $\mathbf{2 0}$ with $\mathbf{2 7 a}$ and $\mathbf{2 7 b}$ and their Relative Stereochemistry Determinations . . . . . . . . . . . . . . . 57

3.19 Boron-Mediated Aldol Reactions of 19a, 3 and $\mathbf{5}$ with Isobutyraldehyde and

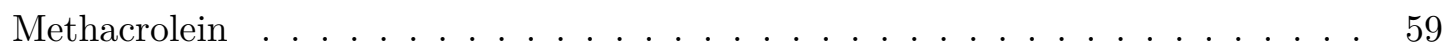

3.20 Possible Decomposition Pathway for 19a in the Work-up Step . . . . . . . . . . 59

3.21 Aldol Reactions of ( $r a c)-3$ with Methacrolein $\mathbf{2 7 a}$ and 4-Bromobenzaldehyde $\mathbf{2 7 d}$ using Different Mediating Agents ${ }^{55,56} \ldots \ldots$. . . . . . . . . . . . . 61

3.22 Titanium-Mediated Aldol Reactions Reported by Ghosh et al. ${ }^{56} \ldots \ldots$. . . . 61

3.23 Aldol Intermediates in the NMR study of the Aldol Reaction of 2-Hexanone . . . 70

3.24 Proposed Mechanism of Aldol Reactions of Diborylated Enolates Derived from $\beta$-Oxygenated Methyl Alkyl Ketones . . . . . . . . . . . . . . . . . . . . 76

3.25 Aldol Reaction of $\mathbf{2}$ with $\mathbf{2 7} \mathbf{a}$ in the NMR Study . . . . . . . . . . . . . 82

3.26 Aldol Reaction of $\mathbf{3}$ with $\mathbf{2 7 a}$ in the NMR study . . . . . . . . . . . . . . 85

3.27 A Goodman's anti-Boat Conformer Responsible for the rOe Correlations of $\mathrm{H}_{7}$ and $\mathrm{H}_{13}$ of Boron-Complexed Aldol Product $44 \ldots \ldots$. . . . . . . . 87

3.28 An Unlikely Hydrogen bond between $\mathrm{O}_{7}$ and $\mathrm{H}_{4}$ in $44 \ldots \ldots \ldots$

4.1 Hoye's RRCM ${ }^{64}$ (Reaction (1)) and Proposed RRCM (Reaction (2) and (3)) . . 97

4.2 Alternative Tethers for Metathesis Reactions . . . . . . . . . . . . . . . 97

4.3 Proposed Rainier and Takeda RCM in the Syntheses of $4 \mathrm{a}$ and $4 \mathrm{~b}^{65,66} \ldots$. . . 98

4.4 Proposed Aldol Reactions for Future Work . . . . . . . . . . . . . . . . . . 99 


\section{List of Tables}

1.1 Aldol Products Generated via Felkin-Anh or Cram-Chelation Control ${ }^{23-28}$. . . . 10

1.2 (-)-Diisopinocampheyl Boron Reagent Mediating Asymmetric Aldol Reactions of Terminal and Internal Enolates with Various Aldehydes ${ }^{32} \ldots \ldots$. . . . . . . . 20

1.3 Masamune's 1,5-Anti-Aldol Reactions for Coupling $\mathrm{C}_{10} \mathrm{C}_{11}$ Bond of Bryostatin ${ }^{35,36} 24$

1.4 Enolizations of Different Acetyl Compounds ${ }^{7} \ldots \ldots \ldots$. . . . . . . . . . 32

3.1 The Use of Commerical and "Homemade" Boron Reagents in the Aldol reactions of Acetone with Methacrolein . . . . . . . . . . . . . . . . . . 38

3.2 Key ${ }^{13} \mathrm{C}$ and ${ }^{1} \mathrm{H}$ Chemical Shifts and nOe Correlations of $2 \ldots \ldots \ldots$

$3.3{ }^{13} \mathrm{C}$ and ${ }^{1} \mathrm{H}$ Chemical Shifts and Key nOe Correlations of $\mathbf{3} \ldots \ldots \ldots$

3.4 Summary of Different Amine Bases Used in NMR Study of Enolization of 2Hexanone . . . . . . . . . . . . . . . . . . . . . 70

3.5 Upfield-Shifting Effect from the Anisotropic Effect of the Phenyl Ring . . . . . . 80

3.6 Different Upfield-Shifting Effects From Flexible to Conformationally Restricted Phenyl Groups for Acryloyl Esters . . . . . . . . . . . . . . . . . 80

3.7 Key ${ }^{1} \mathrm{H}$ Spectral Data and rOe Correlation of $43 \mathrm{~b} \ldots \ldots$. . . . . . . . . . . 84

3.8 Key ${ }^{1} \mathrm{H}$ Spectral Data and rOe Correlations of $44 \ldots \ldots$. . . . . . . . 86

3.9 Aldol Reactions of $\mathbf{2}$ and $\mathbf{3}$ with Various Aldehydes Investigated in the Model Studies . . . . . . . . . . . . . . . . . . . . . 95 


\section{Glossary}

\begin{tabular}{|c|c|}
\hline $\mathrm{ACP}$ & acyl carrier protein \\
\hline app. & apparent \\
\hline aq. & aqueous \\
\hline AT & acyltransferase \\
\hline br & broad \\
\hline $\mathrm{Bu}$ & butyl \\
\hline$t-\mathrm{Bu}$ & tert-butyl \\
\hline $\mathrm{CoA}$ & coenzyme A \\
\hline COSY & ${ }^{1} \mathrm{H}-{ }^{1} \mathrm{H}$ Correlation Spectroscopy \\
\hline CSA & camphorsulfonic acid \\
\hline CSS & chalcone/stilbene synthase \\
\hline $\mathrm{Cy}$ & cyclohexyl \\
\hline $\mathrm{d}$ & doublet \\
\hline DABCO & 1,4-diazabicyclo[2.2.2]octane \\
\hline dd & doublet of doublets \\
\hline DDQ & 2,3-dichloro-5,6-dicyano-1,4-benzoquinone \\
\hline DEB & 6-deoxyerythronolide B \\
\hline DEBS & 6-deoxyerythronolide B synthase \\
\hline$\delta$ & chemical shift in ppm relative to tetramethylsilane $(\delta=0)$ \\
\hline DH & dehydrogenase \\
\hline DIPEA & diisopropylethylamine \\
\hline
\end{tabular}


DIPOTf $B$-trifluoromethanesulfonate diisopinocampheylborane

DMSO dimethyl sulfoxide

dq doublet of quartets

dr diastereomeric ratio

dt doublet of triplets

E1cB unimolecular elimination-conjugate base

ee enantiomeric excess

eq. $\quad$ equivalent(s)

ER enol reductase

ESI electrospray ionisation

Et ethyl

EtOAc ethyl acetate

FAS fatty acid synthase

g $\quad \operatorname{gram}(\mathrm{s})$

h $\quad \operatorname{hour}(\mathrm{s})$

HMBC heteronuclear multiple bond coherence

HMG 3-hydroxy-3-methyl-glutaryl

HP20 styrene-divinyl benzene beads

HR highly reducing

HRMS high-resolution mass spectrometry

HSQC heteronuclear single quantum coherence

$\mathrm{IC}_{50} \quad 50 \%$ inhibitory concentration

Ipc isopinocamphenyl

$(\mathrm{Ipc})_{2} \mathrm{BCl} \quad B$-chlorodiisopinocampheylborane

(Ipc) ${ }_{2}$ BOTf $\quad B$-trifluoromethanesulfonate diisopinocampheylborane

IR $\quad$ infrared

KHMDS potassium bis(trimethylsilyl)amide 


\begin{tabular}{|c|c|}
\hline $\mathrm{KR}$ & ketoreductase \\
\hline $\mathrm{KS}$ & ketosynthase \\
\hline $\mathrm{L}$ & litre(s) \\
\hline LDA & lithium diisopropylamide \\
\hline LiHMDS & lithium bis(trimethylsilyl)amide \\
\hline lit. & literature(s) \\
\hline $\mathrm{Me}$ & methyl \\
\hline $\mathrm{Me}_{4} \mathrm{NBH}(\mathrm{OAc})_{3}$ & tetramethylammonium triacetoxyborohydride \\
\hline $\min$ & minute(s) \\
\hline mol & mole(s) \\
\hline $\mathrm{mMCoA}$ & methylmalonyl-CoA \\
\hline $\mathrm{MT}$ & methyl transferase \\
\hline$\mu \mathrm{g}$ & $\operatorname{microgram}(\mathrm{s})$ \\
\hline $\mathrm{mg}$ & milligram(s) \\
\hline $\mathrm{mL}$ & millilitre(s) \\
\hline NMR & nuclear magnetic resonance \\
\hline nOe & nuclear Overhauser effect \\
\hline NOESY & nuclear Overhauser effect spectroscopy \\
\hline NR & non-reducing \\
\hline $\mathrm{Nu}$ & nucleophile \\
\hline PK & polyketide \\
\hline PKS & polyketide synthase \\
\hline PMB & 4-methoxybenzyl \\
\hline PMP & 4-methoxyphenyl \\
\hline ppm & parts per million \\
\hline $\operatorname{Pr}$ & propyl \\
\hline PR & polyketide reductase \\
\hline
\end{tabular}




\begin{tabular}{|c|c|}
\hline$i-\mathrm{Pr}$ & isopropyl \\
\hline q & quartet \\
\hline $\mathrm{RCM}$ & ring closing metathesis \\
\hline ref. & reference(s) \\
\hline $\mathrm{rOe}$ & rotating frame nuclear Overhauser effect \\
\hline ROESY & rotating frame nuclear Overhauser effect spectroscopy \\
\hline $\mathrm{RRCM}$ & relay ring closing metathesis \\
\hline RT & room temperature \\
\hline s & singlet \\
\hline SAR & structure-activity relationship \\
\hline sat. & saturated \\
\hline $\mathrm{Sn}(\mathrm{OTf})_{2}$ & stannous triflate \\
\hline SN2 & bimolecular nucleophilic substitution \\
\hline $\mathrm{t}$ & triplet \\
\hline TBAF & tetrabutylammonium fluoride \\
\hline TBS & tert-butyldimethylsilyl \\
\hline TBDMS & tert-butyldimethylsilyl \\
\hline TBDPS & tert-butyldiphenylsilyl \\
\hline $\mathrm{td}$ & triplet of doublets \\
\hline TES & triethylsilyl \\
\hline $\mathrm{Tf}$ & trifluoromethanesulfonic acid \\
\hline TLC & thin layer chromatography \\
\hline TMP & 2,2,6,6-tetramethylpiperidine \\
\hline TMS & trimethylsilyl \\
\hline TS & transition state \\
\hline UV & ultraviolet \\
\hline vis & visible \\
\hline
\end{tabular}




\section{Chapter 1}

\section{Background and Introduction}

A brief introduction to polyketide (PK) biosynthesis is outlined in Section 1.1. This gives a preliminary idea about how a PK, such as peloruside A, is synthesized in an organism and the major distinction between biosynthesis and chemical synthesis of PKs. Section 1.2 outlines the discovery and biochemistry of peloruside A whose synthesis can involve a 1,5-anti aldol reaction which forms the basis of this project. Section 1.3 discusses the important discoveries of substratedirected asymmetric aldol reactions and builds towards an understanding of 1,5-anti-induction in aldol reactions. Section 1.4 describes the mechanistic investigations of "propionate-type" and "acetate-type"-aldol reactions and the effort to improve the stereochemcal outcomes of the aldol reactions of $(E)$ - and terminal enolates. An overview of investigations of 1,5-anti-aldol reactions, including two proposed models based on computational modelling and one potential model modified from Abiko's diborylated model, is also outlined.

\subsection{Polyketide Biosynthesis}

The PKs constitute a large family of natural products, biosynthesized by bacteria, fungi, plants and marine animals. Between 5000 and 10000 PKs have been discovered and 1\% of them exhibit significant pharmacological activity that is five times higher than the average activity observed in natural products. ${ }^{8}$ In reality, PKs can be bio-transformed into highly diverse structures, which include fatty acids, aromatic compounds, polypropionates and polyacetates. These highly diverse structures are often complicated and challenging synthetic targets. However, the biosynthetic building blocks are surprisingly simple: propionate and acetate. With the progress of biotechnology, the biosynthetic mechanisms that generate PKs have been gradually revealed and the biosynthetic pathways can sometimes be controlled and re-designed to achieve PK analogues. ${ }^{9}$ Because of the polyol backbone of PKs, the aldol reaction is particularly important for chemical synthesis of PK. In contrast, PK biosynthesis uses Claisen-type condensations followed by asymmetric reduction of the ketones with PK reductases (PR) to achieve the 1,3-polyol structure. 


\subsubsection{Classification of Polyketide Synthases (PKSs)}

Polyketide biosynthesis has many similarities to fatty acid biosynthesis, such as common biological precursors, carrier proteins and enzymes. However, there are key differences between PK and fatty acid biosynthesis. The PKS family can accept a larger range of precursors and generate a variety of chain lengths depending on the organization of the PKS and genetic programming. Furthermore, a fatty acid synthase (FAS) causes a full reduction of each carbonyl group to a methylene after each condensation, whereas a PKS may or may not partially or fully reduce carbonyl groups. ${ }^{9}$ PKSs can be classified into different types based on their architectures and mode of action. The type I PKS class is large, multifunctional and composed of linearly arranged and covalently fused domains, whereas type II PKSs include a series of discrete and usually monofunctional enzymes. The multifunctional enzymes for chalcone synthesis which is an essential biosynthesis for plant are classified as type III PKSs (Figure 1.1. PKSs can be also categorized as iterative and noniterative referring to whether each ketosynthase (KS) domain catalyzes more than one round of elongation. ${ }^{9}$
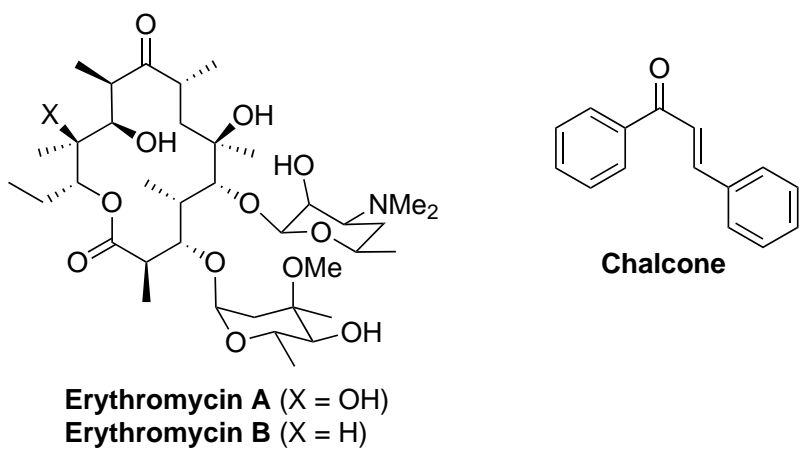

Figure 1.1: Erythromycin and Chalcone

\subsubsection{Biosynthesis of DEBS}

Non-iterative type I PKSs, such as the 6-deoxyerythronolide B synthase (DEBS) and the archetypal erythromycin (1.1) PKS, are large multimodular proteins which are mainly found in prokaryotes and recently have also been found in a protozoan. Twenty years ago, the discovery and sequencing of DEBS opened up the pathway to the understanding of the structure and biochemical function of modular PKSs, and DEBS remains an excellent paradigm to introduce the catalytic mechanism in non-iterative type I PKSs. DEBS is a large (2 MDa) modular multifunctional synthase, capable of catalyzing the multistep assembly of seven polypropionate building blocks (methylmalonyl-CoA (mMCoA)) to form the parent macrocyclic aglycone. Each module catalyzes only one elongation cycle, thus the number of modules corresponds to the number of additional carbonyl units in the PK (for DEBS, six additional and one starting propionate unit are present.) (Fig. 1.2). ${ }^{9}$ 


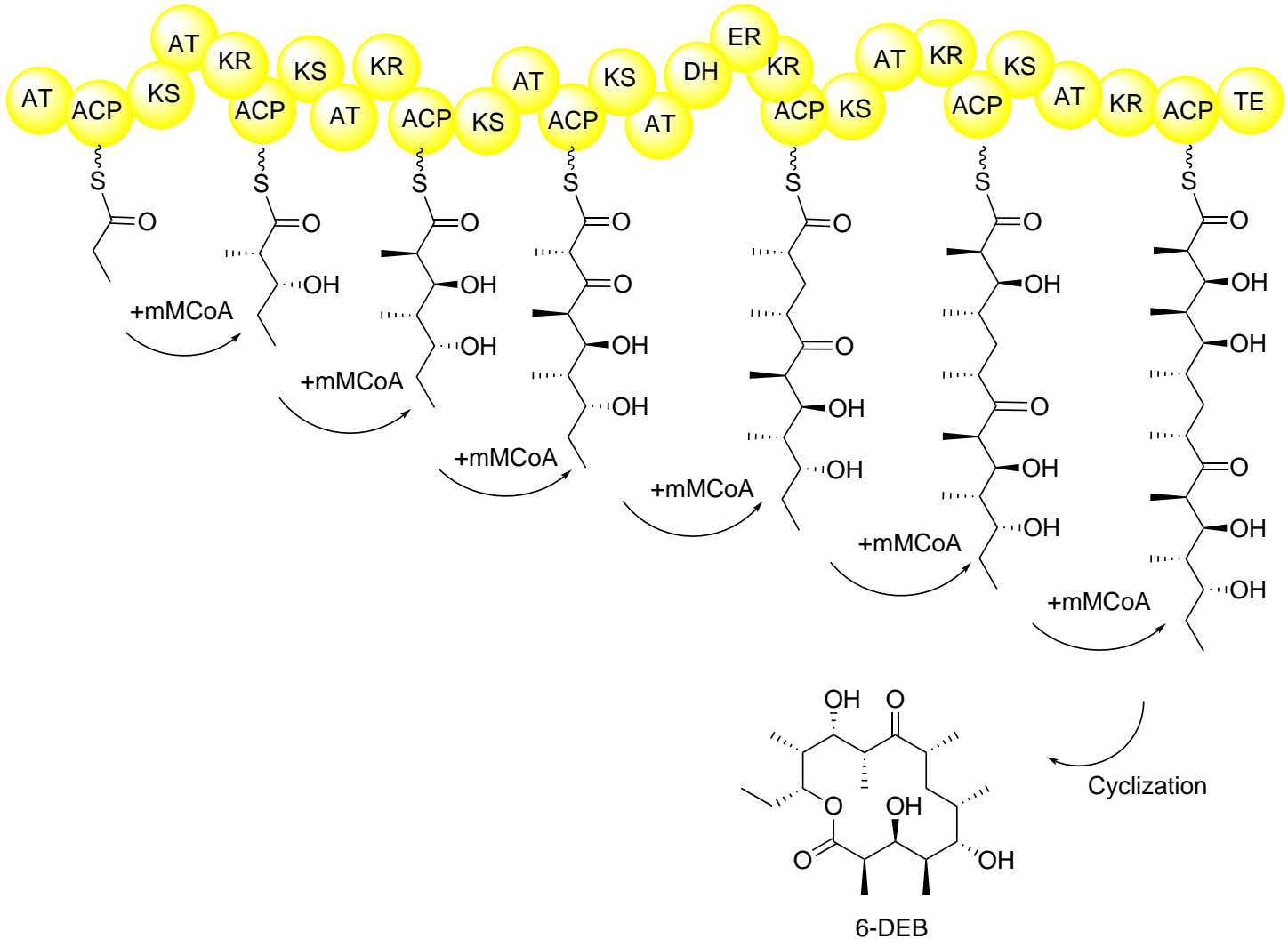

Figure 1.2: Biosynthesis of 6-Deoxyerythronolide B (DEB) by Non-Iterative Type I Polyketide Synthase

\subsection{History and Biochemistry of Peloruside A}

Peloruside A (+)-1 is a secondary metabolite (Fig. 1.3), isolated from a New Zealand marine sponge (Mycale hentscheli) by Northcote and West of Victoria University. ${ }^{1}$ The subsequent investigation by high field NMR studies revealed a polyketide derived structure and its relative stereochemistry. The first total synthesis by De Brabander \& co-workers. was of the unnatural enantiomer of peloruside A, (-)-1, and disclosed the absolute stereochemistry. ${ }^{10}$

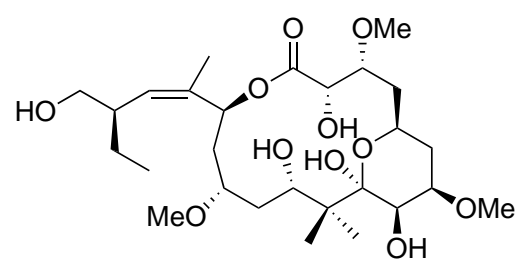

Figure 1.3: Peloruside A (+)-1

Peloruside A shows similar pharmacological effects to the anticancer drug paclitaxel (Taxol) and acts by stabilizing tubulin polymers (microtubules), thus causing apoptosis by arresting the cell cycle at the G2-M checkpoint. The cytotoxicity of peloruside A has been tested in a variety of cell lines, including H441, HL-60, P388 murine leukemic cells, the non-transformed murine 
cell line 32D and ras and bcr/abl oncogene-transformed 32D cells. ${ }^{11}$ The cytotoxicities are similar to other potent microtubule stabilizing agents, such as paclitaxel, with a $\mathrm{IC}_{50}$ value at low nanomolar concentrations. The major restriction in progressing investigations into clinical use of peloruside $\mathrm{A}$ is the limited supply from the sponge source; therefore an efficient synthesis is crucial for its pharmacological and preclinical testing and obtaining structure-activity relationship (SAR) data. ${ }^{12}$ The polyketide-derived structure of peloruside A and the presence of 1,3-dioxygenation indicate that asymmetric aldol reactions would be useful for constructing this 16-membered macrolide.

\subsection{Asymmetric Aldol Reactions}

Aldol chemistry is one of the most powerful and widely used methods for $\mathrm{C}-\mathrm{C}$ bond formation. Tremendous progress has been made during the past few decades in the area of asymmetric aldol chemistry and its application in natural product synthesis. ${ }^{13,14}$ There are many synthetic tools that are used for achieving highly stereoselective aldol reactions. These include chiral auxiliaries, chiral reagents, chiral metal catalysts, chiral organocatalysts, enzyme and antibody catalysts. ${ }^{13,14}$ Substrate directing induction is also an important factor in the asymmetric aldol reaction and allows atom economy, use of inexpensive achiral metal catalysts/reagents and the opportunity to produce a variety of stereoisomers using different substituent(s) at different carbon(s) of the substrate, ${ }^{15,16}$ as described later in this section.

Aldol reactions can be classified according to the structure of the enolate. ${ }^{6}$ Those where the enolate has a carbon chain extension or substituent at the $\alpha$-carbon are called "propionate-type" aldol reactions. Those involving enolates lacking an $\alpha$-substituent are called "acetate-type" aldol reactions. This classification is useful since these two types of enolates exhibit very different reactivities in metal-mediated aldol reactions due to the different conformations of the transition states (TSs) adopted. Both $(E)$ - and $(Z)$-enolates can adopt Zimmerman-Traxler TSs in metal-mediated aldol reactions which lead to anti- and syn-aldol products, respectively. Both the $\alpha$-substituent (R group) of the $(E)$-enolate and the carbon chain $\left(\mathrm{R}_{2}\right.$ group) of the aldehyde are in equatorial positions in chair TS $\mathbf{A}$, which is more stable than chair TS A', thus leading to anti-aldol product (Reaction (1), Scheme 1.1). Analogously, Chair TS B is more stable than chair TS B' since the $\mathrm{R}_{2}$ group of the aldehyde is in an equatorial position in this TS, thus leading to syn-aldol product (Reaction (2), Scheme 1.1). Because of the absence of an $\alpha$-substituent in a methyl ketone-derived enolate, a boat TS is generally favored in the "acetate-type" aldol reaction, but this preference is not pronounced enough to completely dominate, leading to poor stereoselectivity in aldol reactions of methyl ketones. ${ }^{17}$ 
<smiles>[R3]C(=C[B])OC</smiles><smiles>[R2]C=O</smiles>

(E)-enolate

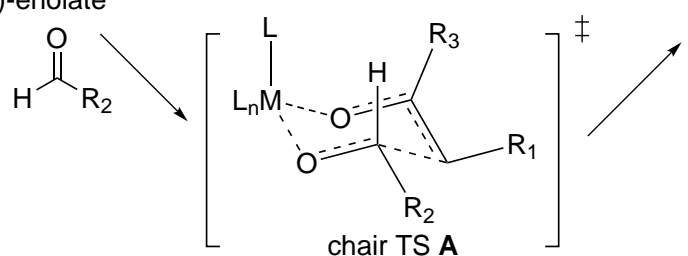

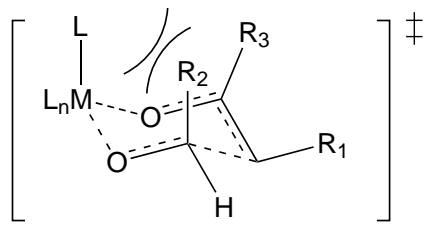

chair TS $A^{\prime}$

(disfavored, leading to a syn product)

$\overline{\mathrm{R}}_{1}$<smiles>[R]C(=O)C([R6])O</smiles>
anti-product

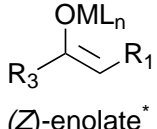<smiles>[R2]C=O</smiles>

(Z)-enolate*<smiles>[R]C(=O)C([R7])C([R6])O</smiles>

syn-product

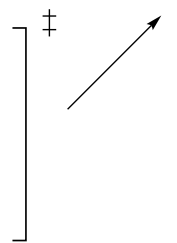

chair TS B

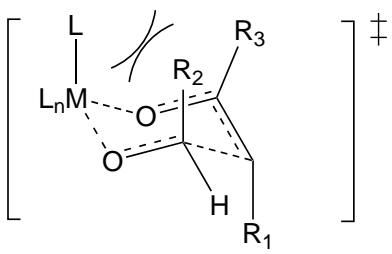

chair TS B'

(disfavored, leading to an anti-product)

Key:

* The designators $\mathrm{E} \& \mathrm{Z}$ would be reversed if the priority of $R_{3}$ became higher than that of OM but the product stereochemistry would remain as shown in this scheme. If $R_{1}=$ hydrogen, it is an "acetate-type" aldol reaction.

If $R_{1}=$ non-hydrogen substituent, it is a "propionate-type" aldol reaction.

$M=$ metal

$L=$ ligands attached to the metal

Scheme 1.1: Transition States and Corresponding Aldol Products of $(E)$ - and $(Z)$-Enolates ${ }^{17}$

The skeleton of the "generic" aldol product (Figure 1.4) has five chiral centers (labelled $\star$ ), including an $\alpha$-substituent, as discussed above, that can potentially affect the stereochemistry of the newly formed alcohol (Red). The following content in this section is an overview of substrate-directed aldol reactions and builds towards an understanding of 1,5-anti-induction via a $\beta$-alkoxy boron methyl enolate. 


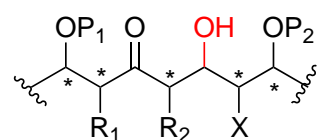

$R_{1}, R_{2} \& R_{3}=$ substituent for the corresponding carbon $P_{1}, P_{2} \& P_{3}=$ protecting group for the alcohol

$\mathrm{X}=\mathrm{OP}_{3}$ or $\mathrm{R}_{3}$

Figure 1.4: Possible Stereoinducing Chiral Centers in the Product of a Substrate-Directed Aldol Reaction

\subsubsection{1,2-Asymmetric Induction in Chiral Aldehydes}

The structure of a chiral aldehyde is an interesting and useful chiral inducing element in asymmetric aldol reactions. ${ }^{18-20}$ The $\alpha$-substituent of an aldehyde is a strong stereocontrolling factor in an aldol reaction due to its close proximity to the carbonyl group. ${ }^{18-20}$ The TS responsible for this stereoinduction is still not fully understood, though a few models can correctly explain most of the experimental results. In 1952, Cram et al. proposed a model of nucleophilic attack on a carbonyl group (Cram model, Scheme 1.2), ${ }^{18}$ in which the carbonyl oxygen orients in-between the two least bulky groups attached to the chiral center. The preferred diastereomeric product will be formed by the approach of the nucleophile from the less hindered face of the carbonyl group. After Cram's initial model, a number of advanced stereochemical models for carbonyl $\pi$-facial selectivity (both ketones and aldehydes) have been proposed. One of these models, the Felkin-Anh model, is widely accepted as explaining the steric, torsional and electronic effects of the $\alpha$-substituent(s) of the carbonyl compound on the stereoselectivity.
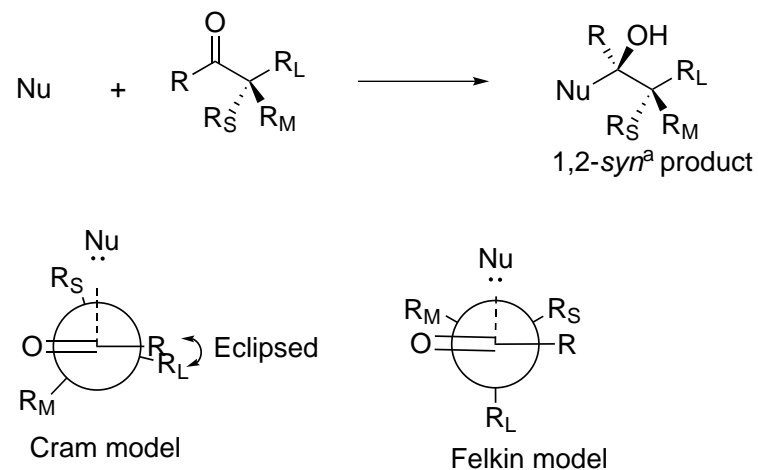

Key:

a. The relative stereochemistry refers to the relative orientation of $\mathrm{OH}$ and $\mathrm{R}_{\mathrm{M}}$. $R_{S}, R_{M}$ and $R_{L}=$ Small, medium and large alkyl groups

Scheme 1.2: Cram and Felkin Models ${ }^{18,19}$

The major drawback of Cram's model is its inability to explain why an increasing stereoselectivity is observed when the bulk of the largest $\alpha$-substituent $\left(\mathrm{R}_{L}\right.$ group $)$ is increased, since 
the $\mathrm{R}$ group is eclipsed with the $\mathrm{R}_{L}$ group. Therefore, Felkin proposed an alternative model in which the $\mathrm{R}_{L}$ group is perpendicular to the plane of the carbonyl group (Felkin model, Scheme 1.2). Furthermore, it was also proposed that the non-hydrogen $R$ group attached to the carbonyl group is larger than the carbonyl oxygen, thus the $\mathrm{R}$ group prefers orienting gauche to the smallest $\alpha$-substituent ( $\mathrm{R}_{S}$ group) so that it is least affected. ${ }^{19}$ The nucleophile can only attack the face of the carbonyl group that is not blocked by the $\mathrm{R}_{L}$ group which leads to the 1,2-syn product. However, this model was not able to explain why the stereoinduction was not reversed when the electrophile was an aldehyde since the $\mathrm{R}$ group (the formyl hydrogen) is not bigger than the carbonyl oxygen. Therefore, the Felkin model was adjusted and extended by Anh and Eisenstein. ${ }^{20}$ They incorporated the Bürgi-Dunitz angle of nucleophile approach to explain why the formyl hydrogen still orients gauche to the smallest $\alpha$-substitutent ( $\mathrm{R}_{S}$ group) during the reaction (Felkin-Anh-Eisenstein model, Scheme 1.3). Since the angle of the nucleophilic attack on the carbonyl $\pi^{*}$ orbital is $107.5^{\circ}$ relative to the plane of the carbonyl group, the proximity of the $\mathrm{R}_{S}$ group can generate less steric interference than $\mathrm{R}_{M}$ or $\mathrm{R}_{L}$ to the incoming nucleophile, thus affording the favorable Felkin-Anh product. Alternatively, if a halogen or other heteroatom ( $\mathrm{X}$ in Scheme 1.3) is present at the $\alpha$-center of the aldehyde, the $\mathrm{C}-\mathrm{X}$ bond is parallel to the formation of $\mathrm{C}-\mathrm{Nu}$ bond because, during the TS of the addition reaction, the LUMO of $\mathrm{C}-\mathrm{X}$ bond can stabilize the development of $\mathrm{C}-\mathrm{Nu}$ bond. This leads to the 1,2-anti product and is termed the Polar Felkin-Anh model (Scheme 1.3).
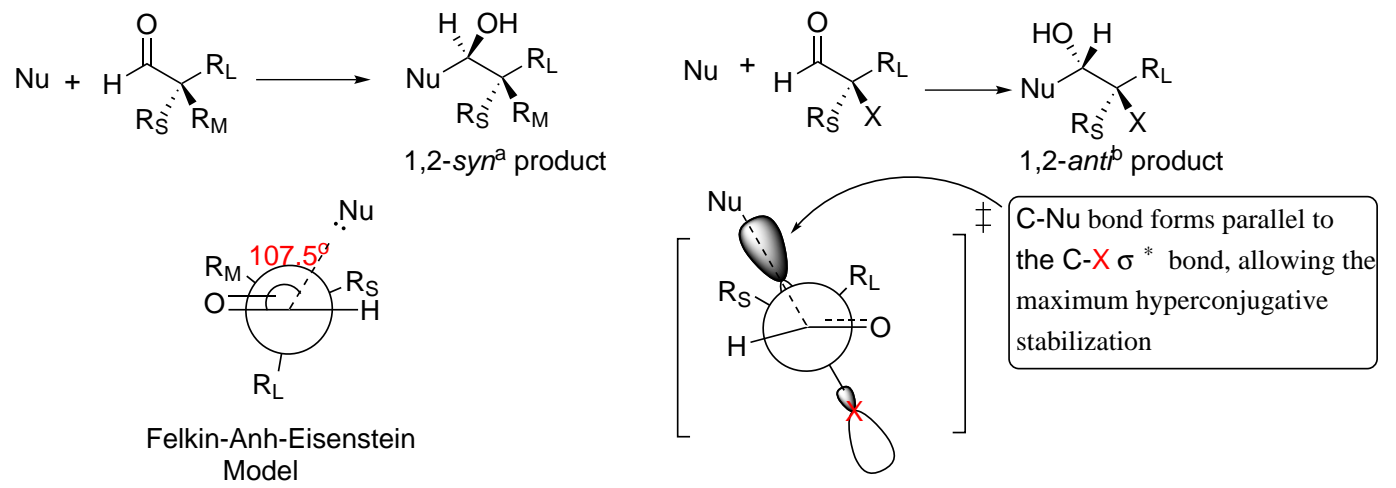

Polar Felkin-Anh Model

Key:

a. The relative stereochemistry refers to the relative orientation of $\mathrm{OH}$ and $\mathrm{R}_{M}$.

b. The relative stereochemistry refers to the relative orientation between $\mathrm{OH}$ and $X$.

$R_{S}, R_{M}$ and $R_{L}=$ Small, medium and large alkyl groups

$X=$ Halogen or other heteroatom

Scheme 1.3: Improved Felkin Model and Polar Felkin-Anh Model ${ }^{20}$

Another model, proposed by Cornforth ${ }^{21}$ and elaborated by Evans ${ }^{22}$ (Scheme 1.4), also explains the formation of the 1,2-anti product. ${ }^{15,16}$ They proposed an antiperiplanar orientation between the halogen and the carbonyl oxygen due to the balancing of the overall dipole moment. The nucleophile will approach the carbonyl from the face that is not blocked by the $\mathrm{R}_{L}$ group 
(Scheme 1.4) and leads to the 1,2-anti product. $^{22}$ The Evans-Cornforth model predicts the same stereochemical outcome as Polar Felkin-Anh model for almost every case. However, recent experimental evidence reported by Evans et al. showed that the Evans-Cornforth model is more able to explain stereoinduction in enolborinate additions to an $\alpha$-alkoxy aldehyde. The scientific deduction in this experiment is beyond the scope of this section. ${ }^{22}$
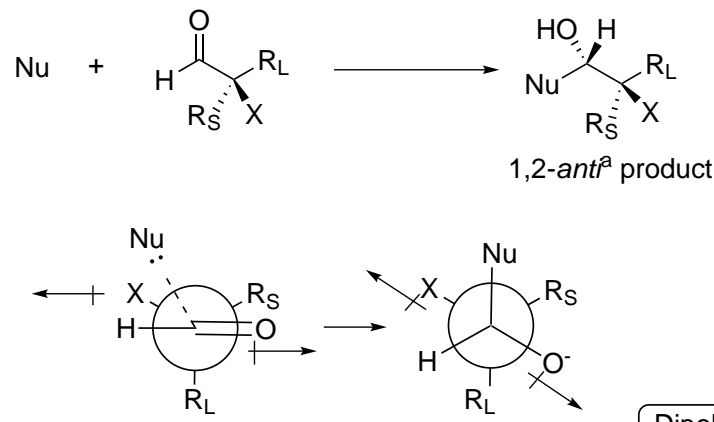

Evans-Cornforth Model

pole moment is balanced before and after nucleophilic addition.

Key:

a. The relative stereochemistry refers to

the relative orientation between $\mathrm{OH}$ and $X$.

$R_{S}$ and $R_{L}=$ Small and large alkyl groups

$X=$ Halogen or other heteroatom

Scheme 1.4: Evans-Cornforth Model for Explaining 1,2-anti-Aldol Chemistry ${ }^{22}$

Both the polar Felkin-Anh model ${ }^{20}$ and the Evans-Cornforth model ${ }^{22}$ describe acyclic TSs of a nucleophilic attack to a carbonyl group. However, if the nucleophilic reactant contains a metal and/or a metal mediating agent is used in this reaction, the anti-Felkin product could be favored instead, generated by a Cram-chelation TS. ${ }^{23}$ As shown in Reaction (1) and (2), Scheme $1.5^{23}$ and Entries 1 and 2, Table 1.1, ${ }^{23-28}$ the nucleophilic addition of the aldehyde with MeMgI favors a Felkin product; in contrast, an anti-Felkin product is generated with $\mathrm{MeTiCl}_{3}$. As shown in Reaction (2), the titanium is chelated by the carbonyl oxygen and the amine of the $\alpha$-substituent of the aldehyde in the reaction and one face of the aldehyde is blocked by the $\alpha$-methyl substituent $\left(\mathrm{R}_{L}\right)$ so that the nucleophile $\left(\mathrm{MeTiCl}_{3}\right)$ can only approach the opposite face, leading to the anti-Felkin product. Therefore, nucleophilic additions to some aldehydes and ketones can favor either Felkin-Anh or Cram chelation models, depending on the choice of the metal reagents (Entries 1 vs. 2, Table 1.1). A similar observation may also happen if the additive reagent(s) is different (Entries 3 vs. 4, Table 1.1). Furthermore, the same metal mediating agent can act by a Felkin-Anh or Cram-chelation TS, depending on the nature of the $\alpha$-substituent of a carbonyl compound. For example, if an $\alpha$-TMSO, TESO or TBSO group is present in the ketone (Entries 5-7), the stereoselectivity of the nucleophilic addition of $\mathrm{Me}_{2} \mathrm{Mg}$ dramatically decreases with an increase of the steric bulk of the silyl protecting group. This large difference was presumably dependent on the ability of the silyloxy group to chelate to the magnesium. For example, $\alpha$-TMSO hardly impedes the chelation but $\alpha$ TIPSO prevents chelation significantly and favours adoption of a Felkin-Anh-type TS (entry 
9). ${ }^{28}$ The electronic factors of the $\alpha$-alkoxy protecting group of the carbonyl compound could also determine the stereochemical outcome. For example, by comparing Entry 10 and 11, an additional 2-methoxy substituent on the $\alpha$-phenyloxy group of the ketone can completely reverse stereochemical outcome (anti-Felkin product). This different stereochemical outcome in Entry 10 is presumably due to a Cram-chelation-like control in which the 2-methoxy substituent of the $\beta$-phenyloxy group participates in the chelation with $\mathrm{Zn}$ instead of the $\beta$-oxygen of the ketone. If a 2 - $t$-butyl group is present on the $\alpha$-phenyloxy group instead, the reaction still favors Felkin-Anh product (Entry 12). Similarly, the other components in the reactions of carbonyl compounds can also reverse the stereochemical outcomes. For example, by comparing Entries 13 and 14, the reductions of the same ketone with the same metal, Sn, but different combinations of reduction reagents can lead to different stereochemical outcomes. If an additional metal mediating agent is present in $\mathrm{NaBH}_{4}$ reduction of a carbonyl compound which favors a Felkin-Anh product, the stereochemical outcome may be reversed by passing through a Cram-chelation TS (Entry 15-19).

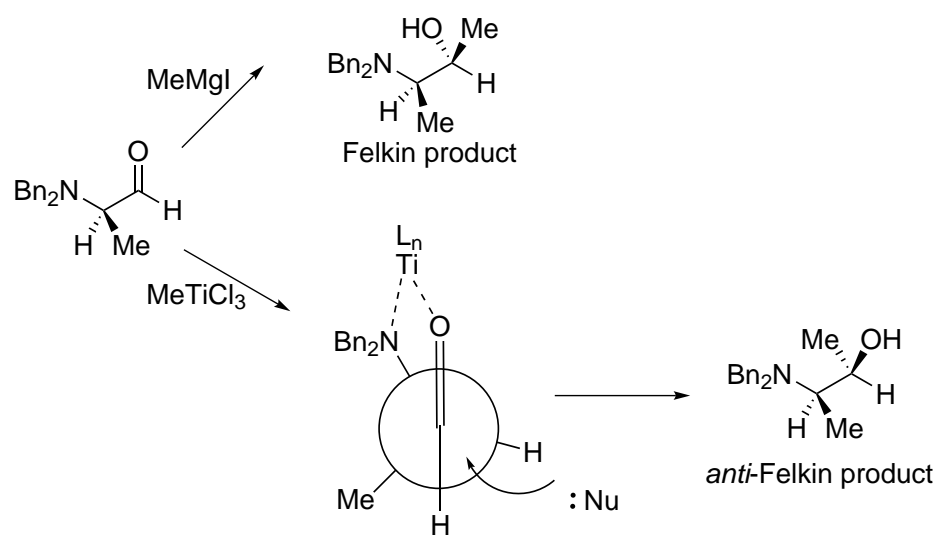

Scheme 1.5: Felkin and Cram-chelation Products Generated from Different Reagents ${ }^{23}$ 


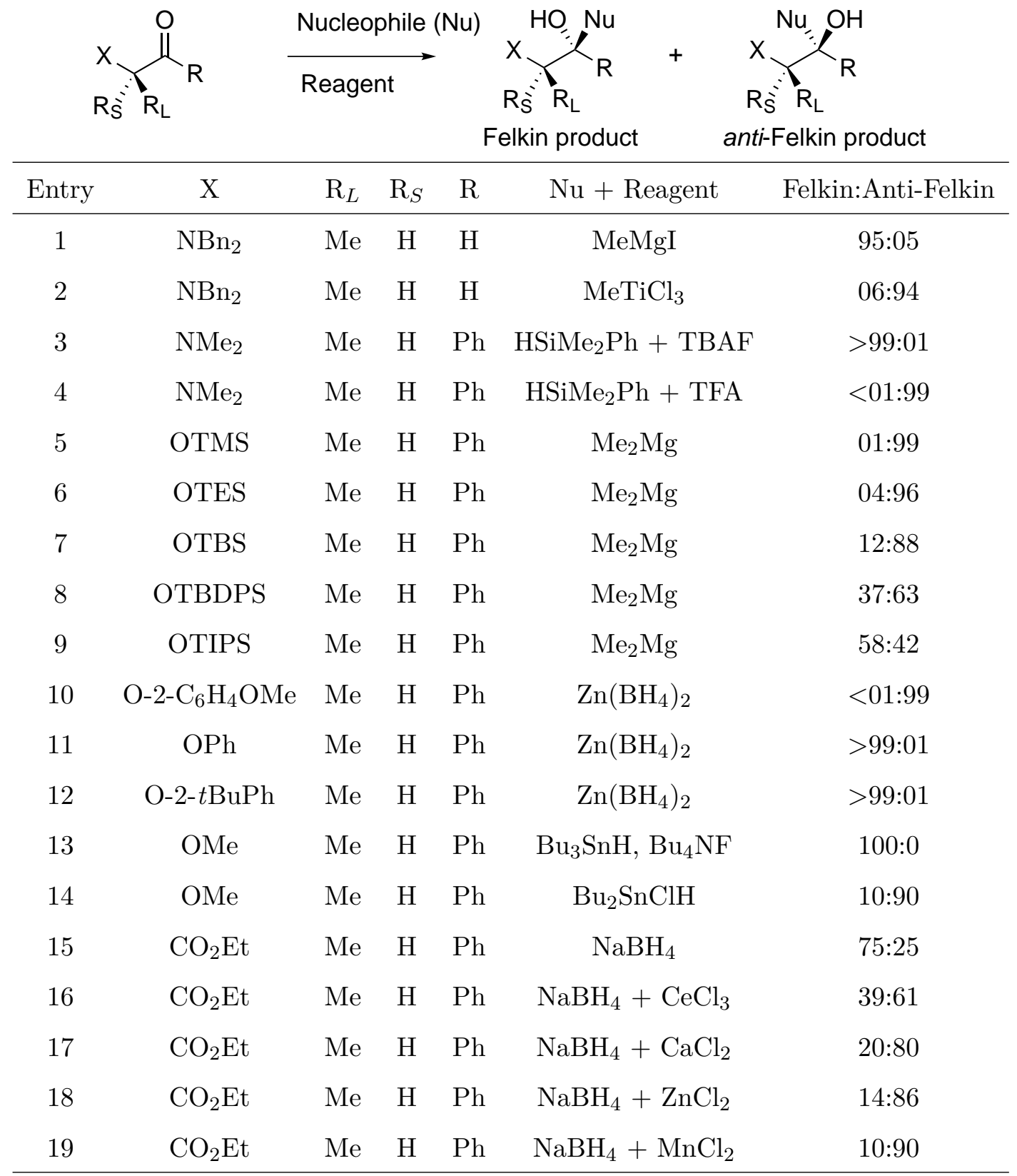

Table 1.1: Aldol Products Generated via Felkin-Anh or Cram-Chelation Control ${ }^{23-28}$

\subsubsection{1,3-Anti Induction in Chiral Aldehydes}

A protected $\beta$-hydroxy group on the aldehyde can also direct the stereochemistry of the newly generated alcohol to produce an anti relationship in the nucleophilic addition (Scheme 1.6). The Cram-chelation model is often used to explain this 1,3-anti induction if a metal mediating reagent is used. As shown in Scheme 1.6, a chair-like conformation is formed after both the $\beta$-oxygen and the carbonyl oxygen chelate to the metal of the mediating agent. The nucleophile prefers to attack the face of the aldehyde that is not occluded by the $\mathrm{R}$ group, which leads to the 1,3-anti product. 


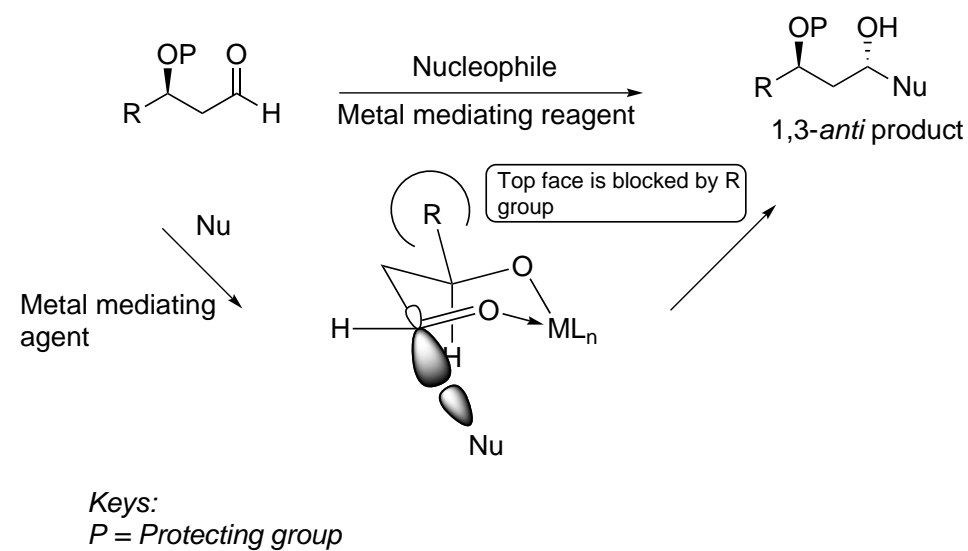

Scheme 1.6: 1,3-Anti Stereoinduction of a Protected $\beta$-Hydroxy Aldehyde in Nucleophilic Addition to a Carbonyl

Surprisingly, Reetz et al. discovered that the use of $\mathrm{BF}_{3} \cdot \mathrm{Et}_{2} \mathrm{O}$ as the Lewis acid in the reaction of $\mathbf{A}$ with $\mathbf{B}$ (Reaction (1), Scheme 1.7) can also lead to a 1,3-anti product with a good stereoselectivity (85:15 anti:syn). ${ }^{29} \mathrm{BF}_{3}$ has only one coordination site, thus Cram-chelation control is not expected for this reaction. An acyclic model, namely the Cram-Reetz model, ${ }^{29}$ is therefore proposed for the reaction, building from Cram's model ${ }^{18}$ for 1,2-asymmetric induction of an aldehyde. Before the nucleophilic attack, the electronegative carbonyl oxygen and $\beta$ oxygen in the aldehyde orient opposite to each other to minimize the overall dipole moment. One face of the aldehyde is blocked by the carbon chain ( $\mathrm{R}$ group) of the aldehyde, thus the nucleophile will prefer attacking the other face which leads to the 1,3-anti product. The major drawback of this model is the presence of an unfavorable eclipsing interaction between the formyl hydrogen and the $\beta$-carbon $\left(\mathrm{C}_{3}\right)$ of the aldehyde. Therefore, an alternative model to explain this 1,3-anti stereoinduction was proposed by Evans et al. ${ }^{30}$ Analogous to Reetz's result, $\mathrm{BF}_{3^{-}}$ mediated Mukaiyama aldol reactions of $\mathbf{C}$ with $\mathbf{D}$ can lead to the 1,3-anti product (Reaction (2), Scheme 1.7) as reported by Evan's group. ${ }^{30}$ Most protecting groups, such as PMB and TBS, for the $\beta$-hydroxy of the aldehydes favor 1,3-anti aldol products but the acetyl protecting group leads to a poor stereoselectivity (43:57 anti:syn). The presence of a $\beta$-chloride in the aldehyde can also direct the formation of 1,3-anti aldol product with a good stereoselectivity (83:17 anti:syn). In the aldol reaction of $\mathbf{E}$ with $\mathbf{C}$ (Reaction (3), Scheme 1.7), the absence of an electronegative group at the $\beta$-carbon of $\mathbf{E}$ leads to poor stereoinduction for the reaction (56:42 anti:syn) which supports the importance of dipole-dipole interaction for the stereoinduction. However, to avoid the unfavorable eclipsing interaction between the formyl hydrogen and $\beta$ carbon of the aldehyde in the Cram-Reetz model, ${ }^{29}$ Evans proposed a revised model in which the $\beta$-carbon is perpendicular to the plane of the carbonyl group. ${ }^{30}$ Among the three models described by Evans (F, $\mathbf{G}$ and $\mathbf{H}$ in Reaction (2), Scheme 1.7), model $\mathbf{F}$ is the most favored one. In model $\mathbf{G}$, the terminal carbon chain of the aldehyde ( $\mathrm{R}$ group) has a gauche interaction with the formyl hydrogen compared to the staggered conformation in model $\mathbf{F}$, thus model $\mathbf{G}$ is less stable. Because of the minimized overall dipole moment in model $\mathbf{F}$, model $\mathbf{H}$ is also less stable than model $\mathbf{F}$. Therefore, model $\mathbf{F}$ has the most favored overall dipole moment and non-bonded 
interactions, leading to a 1,3-anti product. ${ }^{30}$<smiles>C[C@H](CC=O)OCc1ccccc1</smiles>

A

$$
\mathrm{F}_{3} \mathrm{~B}
$$

D

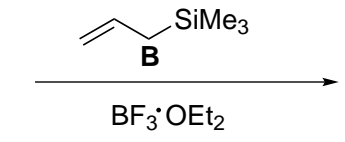

$\mathrm{BF}_{3}^{\mathrm{R}}$<smiles>C=CCC(O)CC(C)COCc1ccccc1</smiles>

1,3-antip product (85:15 anti:syn)<smiles>[X]C(CCc1ccccc1)CC(O)CC(O)C(C)C</smiles>

(1)

(2)

$$
\begin{array}{ll}
\mathrm{X}=\text { OPMB } & 81: 19 \\
\mathrm{X}=\text { OTBS } & 73: 27 \\
\mathrm{X}=\text { OAC } & 43: 57 \\
\mathrm{X}=\mathrm{Cl} & 83: 17
\end{array}
$$

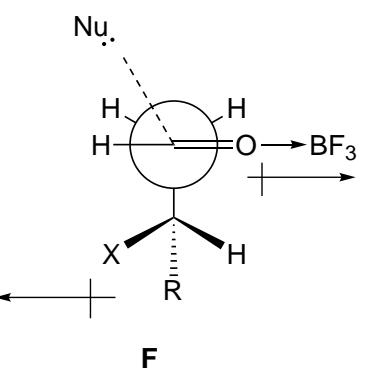

Evans Model

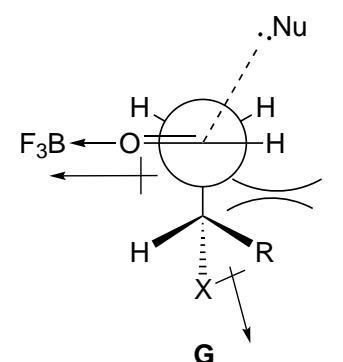

disfavored, leading to $13-s y n$ prc

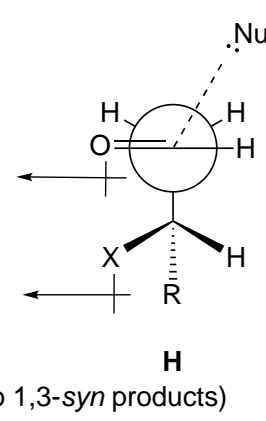

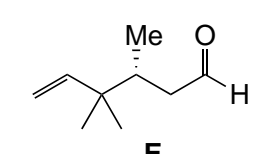

E

$$
\text { Key: }
$$

$P=$ Protecting group

$R=$ Alkyl group
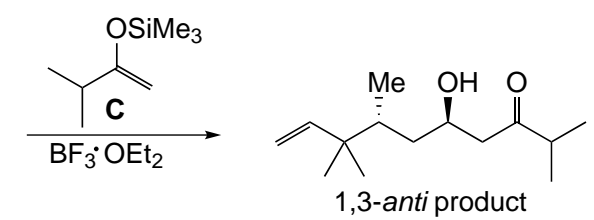

(56:42 anti:syn)

Scheme 1.7: Boron Trifloride-Mediated 1,3-Anti Allylation and Aldol Reactions of Aldehydes via Acyclic Transition States ${ }^{29,30}$ 


\subsubsection{1,2- and 1,3-Merged Asymmetric Induction in Chiral Aldehydes}

According to the Felkin-Anh and Evans 1,3-anti models (Scheme 1.3 and 1.4), when both the $\alpha$ - and $\beta$-substituents are present in the chiral aldehyde, these two stereoinducting factors are expected to either reinforce (matched induction) or counteract (mismatched induction) each other. However, Evans' group showed that these merged asymmetric inductions are more complicated and both "matched" and "mismatched" aldehydes can give good stereoinductions to favor different stereoisomeric forms of the products. ${ }^{15}$ Selected data from Evans' lab is shown in Scheme 1.8. In general, the "matched" aldehyde favors the Felkin product, irrespective of the particular $\beta$-hydroxy protecting group or the steric influences of the silylenol ether (Entries 1-3, Reaction (1), Scheme 1.8). In contrast, the "mismatched" aldehyde favors either Felkin or anti-Felkin products depending on the choice of silyl enol ether (Reaction (2), Scheme 1.8). For example: the syn,syn-Felkin product $\mathbf{C}$ becomes dominant when a large $\mathrm{R}$ group is present in the trimethylsilyl (TMS) enol ether (Entries 1 and 2). With a less sterically bulky enol ether, the 1,3-anti control from the $\beta$-protected hydroxy group of the aldehyde predominates to afford the anti-Felkin (syn,anti-) aldol product $\mathbf{D}$ (Entries 5 and 6 ). The underlying mechanisms for these "mismatched" stereoinductions are not fully understood although Evans' group proposed two stereochemical models (synclinal and antiperiplanar models) for these 1,2- and 1,3-stereoinductions (Scheme 1.9). ${ }^{15}$ If the $\mathrm{R}$ group of the silylenol ether is small, such as a methyl group, the nonbonded interaction between the $\mathrm{R}$ group and the $\mathrm{BF}_{3}$ is attenuated in synclinal models and the carbon backbone of the TS prefers adopting a zig-zag conformation so that the interaction between the nucleophile and the $\alpha$-substituents of the aldehyde is largely reduced and the Felkin-control will be diminished. Furthermore, synclinal model A benefits from the overall dipole minimization, therefore 1,3-anti control is dominant and leads to the anti-Felkin product if the $\mathrm{R}$ group of the silylenol ether has less steric demand. In contrast, if $t$ - $\mathrm{Bu}$ is the $\mathrm{R}$ group of the silylenol ether, the nonbonded interaction between TMS or $t$ - $\mathrm{Bu}$ and $\mathrm{BF}_{3}$ is so large that the nucleophile prefers orientating the bulky groups (TMS and $t$-Bu) away from $\mathrm{BF}_{3}$ and leads to antiperiplanar models $\mathbf{C}$ and $\mathbf{D}$. Because the $\alpha$-methyl group of the aldehyde in model $\mathbf{C}$ has disfavored interaction with the nucleophile and block the Bürgi-Dunitz angle of nucleopile attack, the Felkin product is formed predominately from model $\mathbf{D}$.

By changing to a lithium enolate, anti-Felkin product $\mathbf{F}$ becomes more dominant and interestingly, the larger the $\mathrm{R}$ group in the enolate, the better stereoinduction towards product $\mathbf{F}$ (Entries 1-3, Reaction (3), Scheme 1.8). 


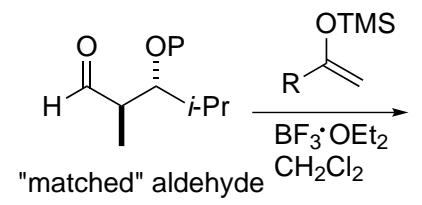

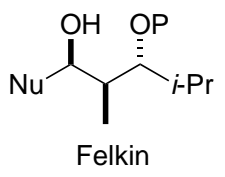<smiles>CCCC(C)C([OH2+])C(C)C(O)C#N</smiles>

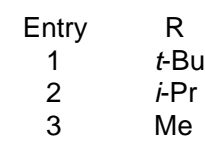

Felkin : Anti-Felkin (yield,\%)

$\begin{array}{cl}\text { P=PMB } & \text { P=TBS } \\ 99: 1(94) & 99: 1 \quad(91) \\ 98: 2(91) & 95: 5(93) \\ 97: 3(86) & 71: 29(85)\end{array}$

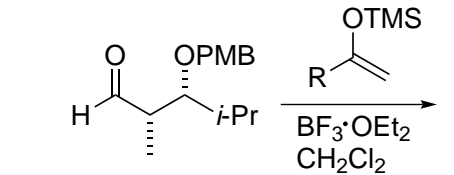

"mismatched" aldehyde

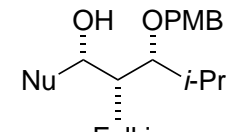

Félkin

C

kin : Anti-Felkin (yield,\%)

$\mathrm{P}=\mathrm{PMB}$

96:4 (89)

$88: 12(75)$

$56: 44$ (98)

$32: 68$ (86)

$17: 83(82)$

$6: 94 \quad(92)$

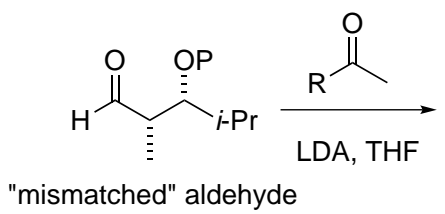

$\overbrace{\equiv}^{\text {Feelkin }} \underset{i-P r}{O H}$

E

Felkin : Anti-Felkin (yield, \%)

$\mathrm{P}=\mathrm{PMB}$

$11: 89(71)$

$14: 86(95)$

22:78 (73)

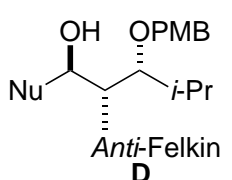

(2)<smiles>CCCC(=O)PC#N</smiles>

Scheme 1.8: Merged 1,2- and 1,3-Asymmetric Induction in Mukaiyama and Lithium-enolate Aldol Reactions According to Evans et al. ${ }^{15}$ 


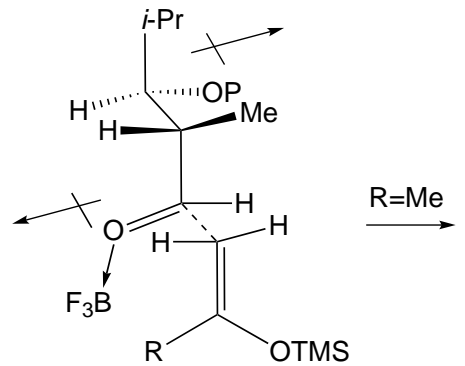

A

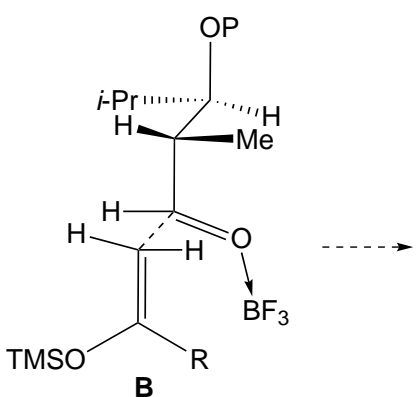

B

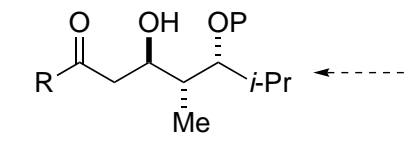

Anti-Felkin Product (1,3-anti induction dominates)

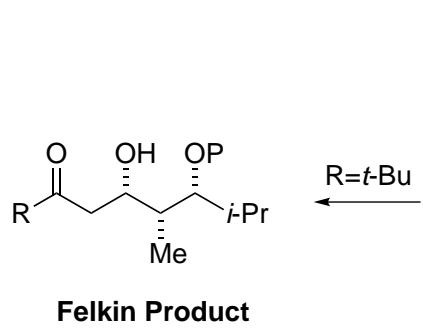

Felkin Product

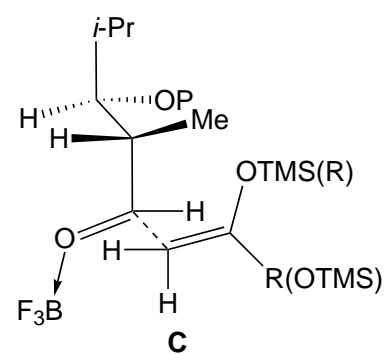

(R)TMSO

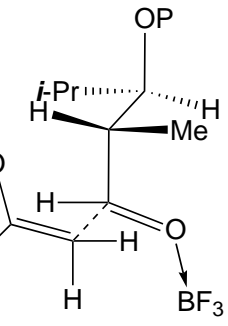

D

Antiperiplanar Models

Synclinal Models

Scheme 1.9: Evans' Models for Explaining 1,2- and 1,3-Asymmetric Induction of "Mismatched" Aldehyde in Mukaiyama Aldol Reaction

\subsubsection{1,4-Syn and Anti Induction in Aldol Coupling of (Z)-Enolates}

Using a chiral internal enolate ( $\alpha$-substituted enolate) or a chiral auxiliary attached internal enolate to induce stereoselective aldol reactions is very popular in natural product synthesis. This chemistry has been studied heavily and there are a variety of choices with various metal mediating agents. For example, recently, Menche's group reported the preparation of 1,4-synor anti-aldol products from $(Z)$-enolates, depending on the choice of metal mediating agents (Scheme 1.10): ${ }^{31}$

- The presence of $\mathrm{TiCl}_{4}$ in TSs $\mathbf{A}$ and $\mathbf{A}^{\prime}$ means the $\beta$-oxygen of the enolate is able to chelate to the titanium so that the $\mathrm{C}_{4}$-substituent of the enolate is closer to the six-membered ring and participates in the stereoinduction (Reaction (1)). Because both the methyl group attached to $\mathrm{C}_{4}$ and the $\beta$-hydrogen point away from the six-membered ring in TS A, thus TS A is lower in energy than TS $\mathbf{A}^{\prime}$ and affords the 1,4-syn product as the major product.

- With boron, because the $\mathrm{B}-\mathrm{O}$ bond is short (1.4 $\AA$ ) and similar in length to a $\mathrm{C}-\mathrm{C}$ bond, the six-membered ring in the TS is more compact, thus the $\beta$-oxygen of the enolate is able to form a hydrogen bond with the formyl hydrogen ${ }^{6}$ (see Goodman's model on page 26) in TSs $\mathbf{B}$ and $\mathbf{B}$ ' to contribute some stabilization to the otherwise disfavored "boat" conformations (Reaction (2)). Analogous to the differences between TS A and A', the methyl substituent at the $\mathrm{C}_{4}$ of the enolate can also point away from the six-membered ring in TS B but not in TS B'. Furthermore, instead of having a disfavored steric interaction between the terminal carbon chain ( $\mathrm{R}_{2}$ group) of the enolate and the substituent (L group) of the boron reagent in TS B', a much smaller interference between the $\beta$-hydrogen and 
the L group exists in TS B. Thus the more stable TS B leads to a 1,4-syn product.

- When LiHMDS is used as the base in the aldol reaction of the same ketone and aldehyde to that described above, a 1,4-anti-aldol product is formed and the TS was proposed to be chair-like, shown as TS $\mathbf{C}$ in Reaction (3). It is assumed that there is no chelation between the $\beta$-oxygen of the enolate and lithium or a formyl hydrogen bond, thus the $\mathrm{C}_{3}-\mathrm{C}_{4}$ bond of the enolate can rotate and the carbon chain ( $\mathrm{R}_{3}$ group) prefers to orientate itself away from the six-membered ring. TS $\mathbf{C}$ is more stable than any others due to its chair conformation and the methyl substituent at $\mathrm{C}_{4}$ of the enolate pointing away from the six-membered ring. 

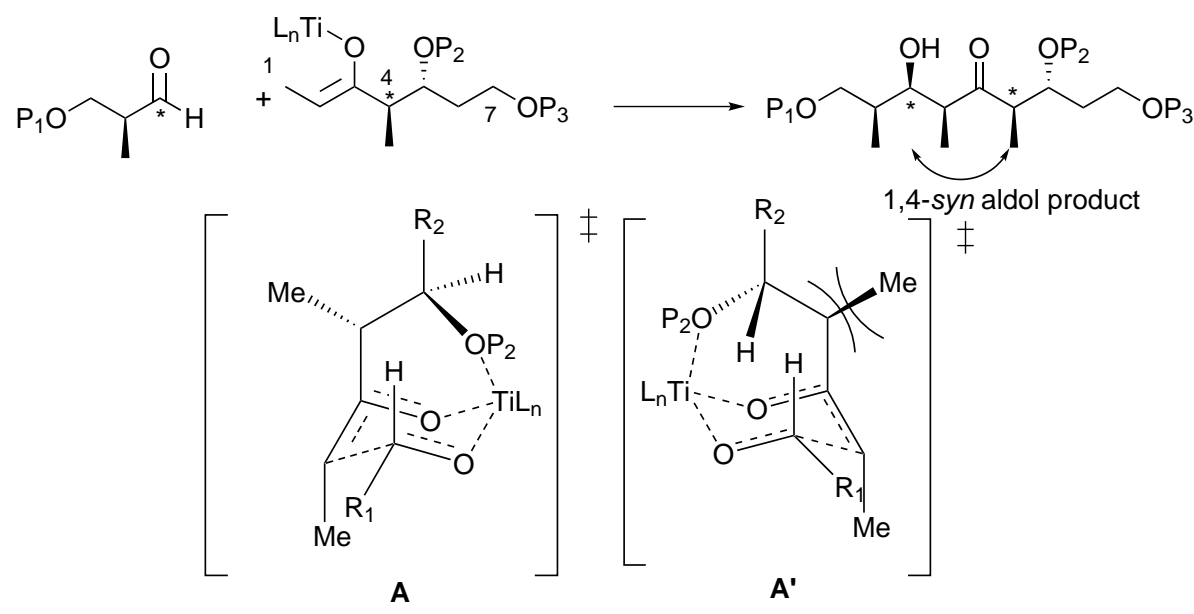

$\overbrace{*}^{H}$
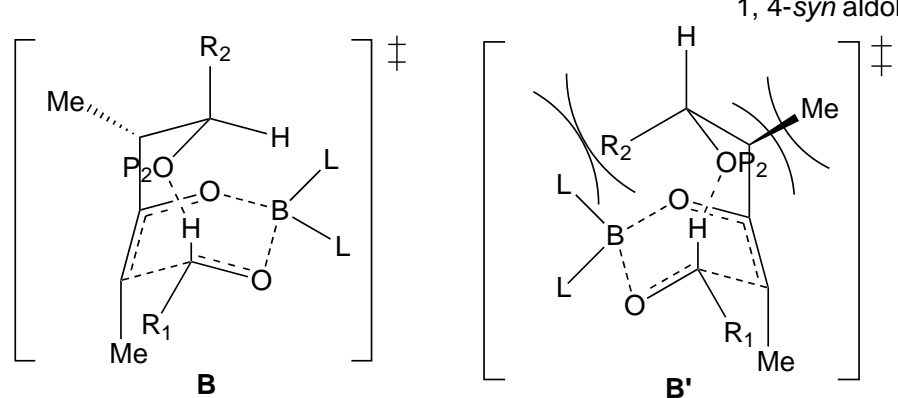

$\overbrace{*}^{\|}$

$$
[
$$

Key:

$P_{1}, P_{2}$ and $P_{3}=$ Protecting groups

$R_{1}=\mathrm{P}_{1} \mathrm{O} \overbrace{}^{\xi_{2}} R_{2}=\mathrm{P}_{3} \mathrm{O} \Upsilon^{z_{2}}$

C

1, 4-anti aldol product

Scheme 1.10: 1,4-Syn and Anti-Induction ${ }^{31}$ 


\section{4 "Propionate-Type" and "Acetate-Type" Aldol Reactions and 1,5-Anti Stereoinduction}

\subsubsection{Transition States of Acetate- and Propionate-Type Aldol Reactions}

Boron-mediated propionate-type aldol reactions combined with the action of a covalent attached chiral auxiliary, allows the regio- and stereoselective formation of aldol products. In contrast to the other metal enolates, the boron-oxygen $(1.4 \AA)$ bond in boron enolates is relatively short and, in reaction with an aldehyde, affords a tight six-membered cyclic TS. This maximizes the 1,3- and 1,4-diaxial interactions in chair and boat TSs, respectively, and initiating and enhancing other structural and electronic factors that influence stereoinduction of the substrates, such as the effect from the chiral auxiliary and the formation of a Goodman hydrogen bond in the boat TS (page 26).

In 1990, Paterson's group reported stereoselective aldol reactions of different enolates can be achieved with (+)- and (-)-(Ipc) $)_{2}$ BOTf/Cl. ${ }^{32}$ Interestingly, in their ${ }^{1} \mathrm{H}$ NMR study of the enolizations of diethylketone, both $(E)$ - and $(Z)$-enolates were detected, depending on the combination of $(+)$ - or $(-)-(\mathrm{Ipc})_{2} \mathrm{BOTf} / \mathrm{Cl}$ and the amine base used in the enolizations. For example, as shown in Reactions (1) and (2), Scheme 1.11, when (-)-(Ipc) ${ }_{2} \mathrm{BOTf} / i$ - $\mathrm{Pr}_{2} \mathrm{NEt}$ was used, $>97 \%$ stereoselectivity in favor of the $(Z)$-enolate was observed whereas the $(E)$-enolate derived from the diethyl ketone was formed in moderate excess $(E: Z=80: 20)$ with (-)-(Ipc) ${ }_{2} \mathrm{BCl} / \mathrm{Et}_{3} \mathrm{~N}$ which explains the moderate diastereoselectivity of the following aldol reaction with methacrolein $(80: 20$ anti:syn). Furthermore, only the $(Z)$-enolate derived from the diethyl ketone can induce a good enantioselectivity ( $80 \%$ ee) whereas $<20 \%$ ee was obtained from the $(E)$-enolate (Reaction $(2)$, Scheme 1.11).

The reagent system (-)-(Ipc) ${ }_{2} \mathrm{BOTf} / i$ - $\mathrm{Pr}_{2} \mathrm{NEt}$ was used in a variety of acetate-type aldol reactions, however only moderate ee (53-73\%) was obtained in most of the reactions (Entry 1-4 and 6, Table 1.2). ${ }^{32}$ When the ketone is able to form either the internal ( $\alpha$-substituted enolate) or terminal enolate ( $\alpha$-unsubstituted enolate), the regioselective enolization is poor, which only moderately favors the terminal enolate (Entry 7) unless there is a large steric difference between the two $\alpha$-carbons (Entry 5). Surprisingly, it was discovered that the opposite enantiotopic face of the aldehyde was attacked by the terminal enolate compared with the internal enolate (Compare Entries 1-7 with 8 and 9). Based on this observation, Paterson et al. proposed that the acetate-type aldol reaction favors a twist-boat TS instead of a Zimmerman-Traxler TS (Scheme 1.12) since the Ipc substituent of the boron reagent can avoid an unfavorable steric interaction with $\mathrm{R}_{1}$ group of the methyl enolate and no strong steric interference was expected between the other Ipc substituent and the $\alpha$-hydrogen $(\mathbf{A})$. In contrast, the aldol reaction of the $(Z)$-enolate favors a Zimmerman-Traxler TS (B) to minimize the more unfavorable interaction between the $\alpha$-methyl group and the Ipc substituent. 

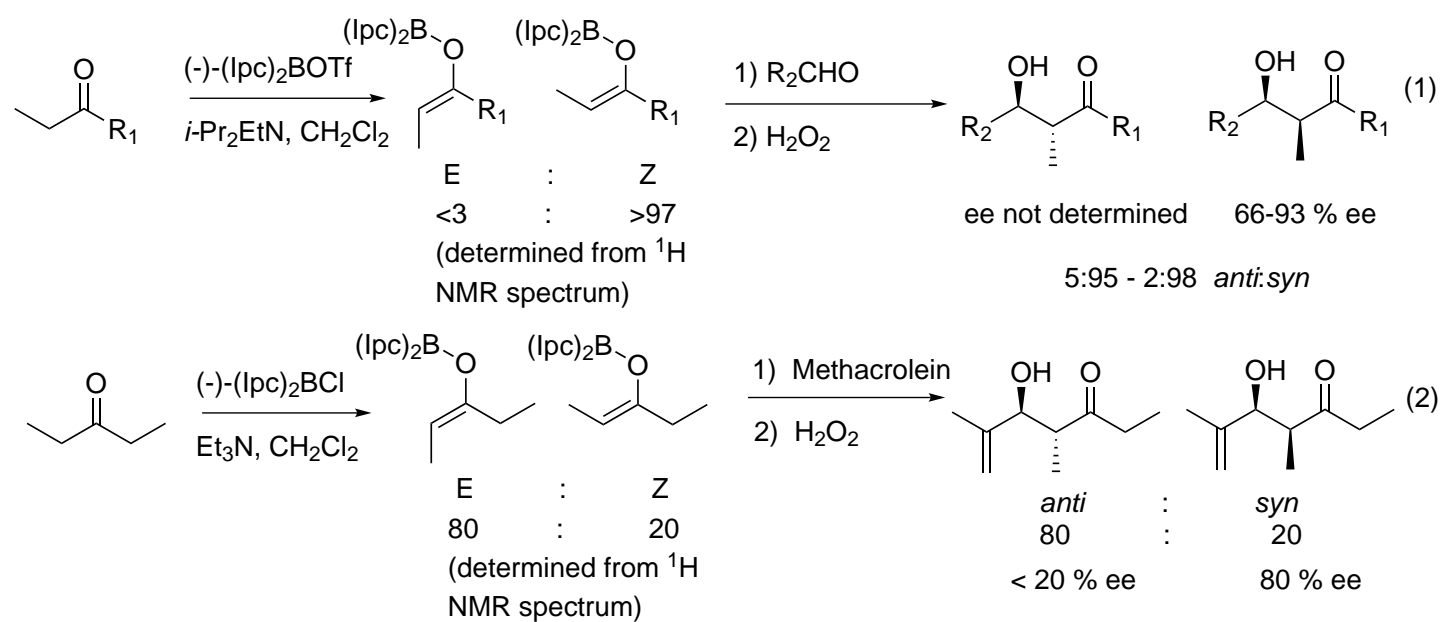

Scheme 1.11: Asymmetric Enolizations and Aldol Reactions with (-)-(Ipc) ${ }_{2}$ BOTf and (-)(Ipc) ${ }_{2} \mathrm{BCl}$ According to Paterson ${ }^{32}$
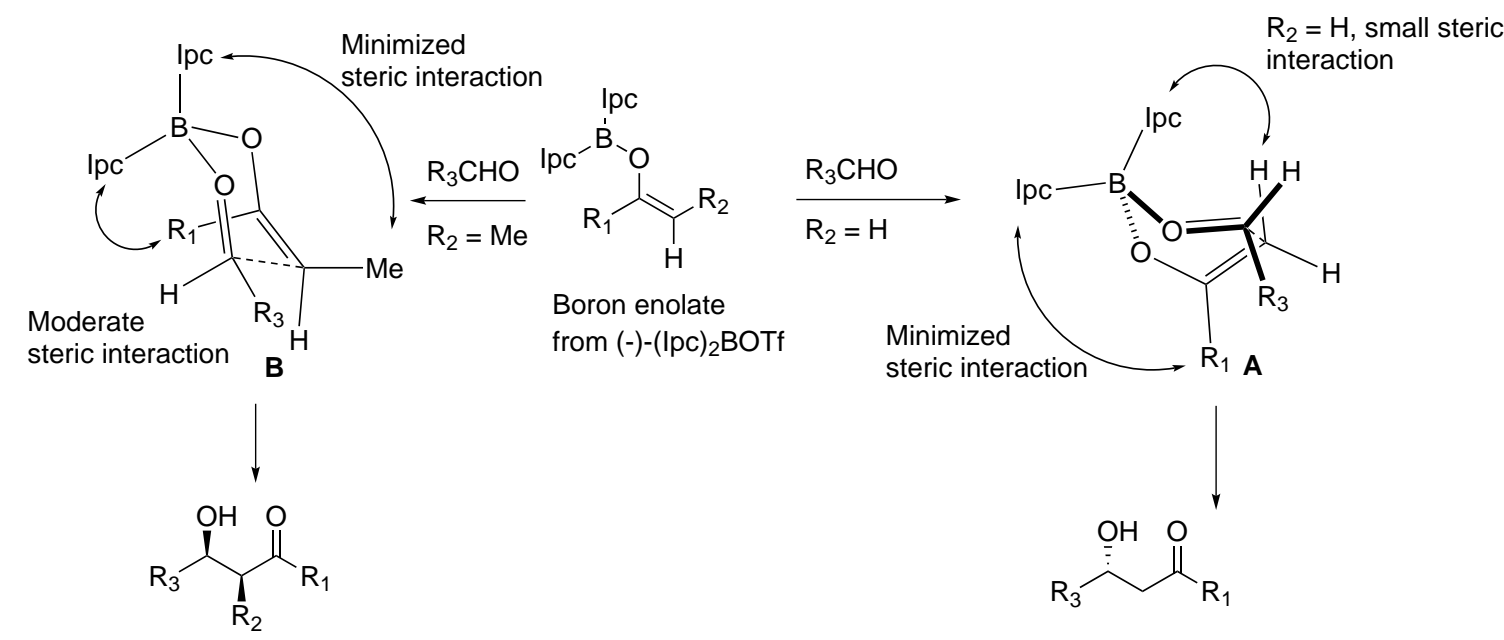

Scheme 1.12: Paterson's Models for Diisopinocampheyl Boron Mediated Asymmetric Aldol Reactions $^{32}$

In summary, this analysis showed that the formation of an $(Z)$-enolate from the ethyl ketone can be achieved in an excellent diastereoselectivity using (-)-(Ipc) $)_{2} \mathrm{BOTf} / i$ - $\mathrm{Pr}_{2}$ EtN, which also leads to the excellent stereochemical outcome in the following aldol reaction. In contrast, the application of $(-)-(\mathrm{Ipc})_{2} \mathrm{BCl} / \mathrm{Et}_{3} \mathrm{~N}$ to the corresponding aldol reaction leads to a moderate diastereoselectivity which can be explained in terms of the poor kinetic resolution in the enolization of the ethyl ketone to form the $(E)$-enolate. However, the low enantio-induction of the (-)-(Ipc) ${ }_{2} \mathrm{BCl} / \mathrm{Et}_{3} \mathrm{~N}$ system in the aldol reaction of the $(E)$-enolate remained unsolved by Paterson et al. ${ }^{32}$ The terminal enolate favors attacking the opposite enantiotopic face of the aldehyde compared to $(E)$ - and $(Z)$-enolates, which is because an unconventional twist-boat TS is adopted in this reaction.

A series of conformational calculations were reported by Goodman and Paton ${ }^{17}$ that attempt to explain these interesting aldol reactions. In the computed TSs (Figured 1.5 and 1.6), the $(E)$ - 


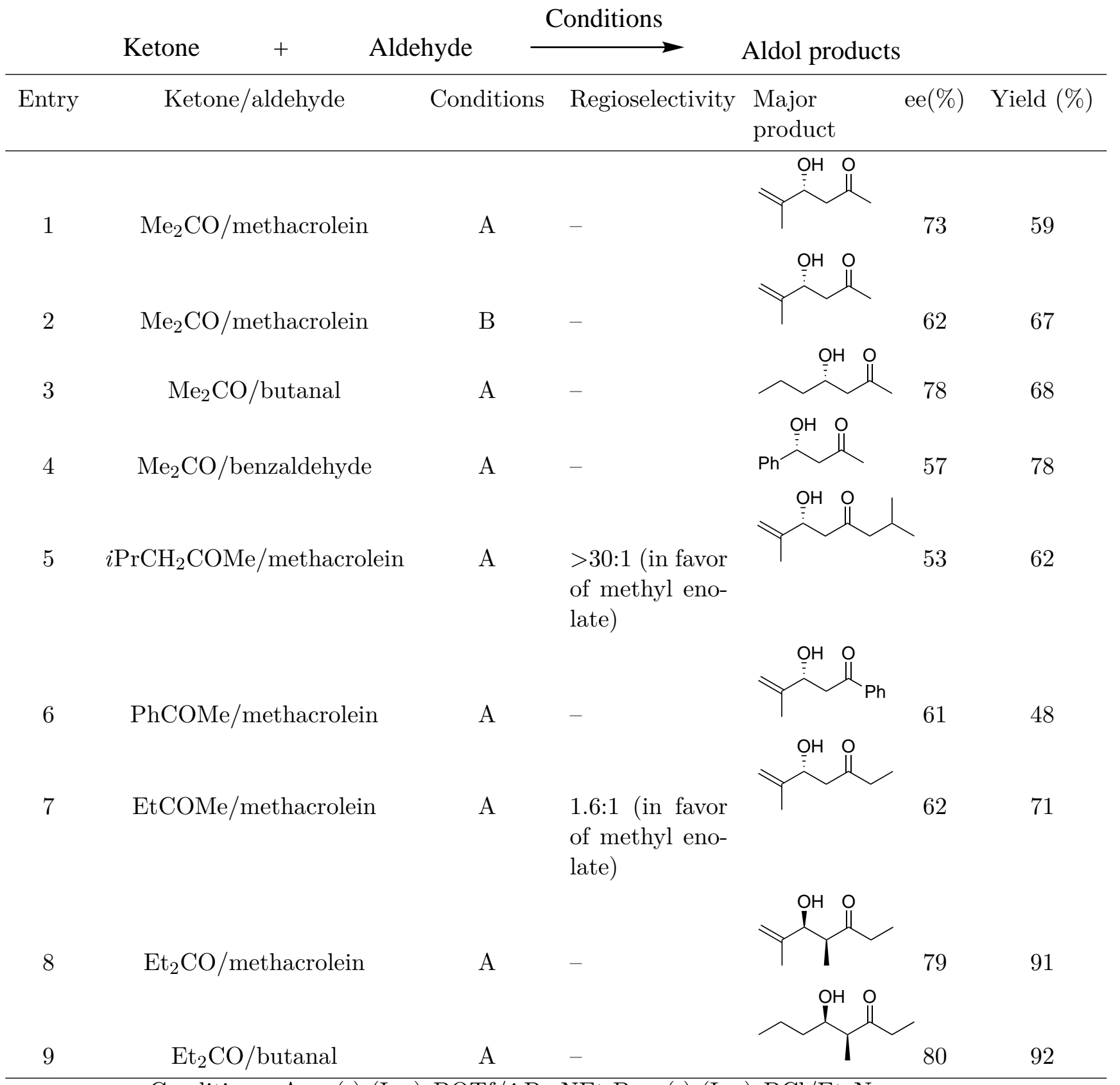

Conditions: $\mathrm{A}=(-)-(\mathrm{Ipc})_{2} \mathrm{BOTf} / i$ - $\mathrm{Pr}_{2} \mathrm{NEt} \mathrm{B}=(-)-(\mathrm{Ipc})_{2} \mathrm{BCl} / \mathrm{Et}_{3} \mathrm{~N}$

Table 1.2: (-)-Diisopinocampheyl Boron Reagent Mediating Asymmetric Aldol Reactions of Terminal and Internal Enolates with Various Aldehydes ${ }^{32}$ 
and $(Z)$-enolates were derived from butan-2-one, the terminal enolate from acetone, and methyl groups were used as boron substituents to simplify the calculation. The chair was found to be the predominant TS for the aldol reaction of the $(Z)$-enolate, as the boat TSs are destabilized by strong 1,4-interactions between the axial ligand of the boron reagent and the $\alpha$-methyl group, leading to the syn-adduct being favored (100:0) in the calculation. In contrast, for the $(E)$-enolate, the chair and boat conformations are energetically similar in the TSs and the boat A conformation is lowest energy (Figure 1.5). Furthermore, calculation of the terminal enolate indicated that the boat $\mathbf{A}$ conformation was again preferred (Figure 1.6). In both latter cases, the boat conformations relieve the 1,3-diaxial interactions and the resulting 1,4-steric interactions are not strong. However, the relative energies of this preferred boat $\mathbf{A}$ conformation and other forms are not pronounced enough to completely dominate, therefore, moderate diastereoand enantioselectivities result from the aldol reactions of an $(E)$-enolate and a terminal enolate, respectively. Thus, in addition to the poor kinetic resolution in the formation of the $(E)$-enolate reported by Paterson et al. ${ }^{32}$ this study proposed that the preferred boat TS of the $(E)$-enolate is not completely dominant, leading to the moderate diastereoselectivity of the aldol reaction. However, in contrast to the twist-boat TS of a terminal enolate proposed by Paterson et al., ${ }^{32}$ a boat TS is favored in the Goodman's calculation.

The poor enantio-induction by $(\mathrm{Ipc})_{2} \mathrm{BCl} / \mathrm{Et}_{3} \mathrm{~N}$ in aldol reactions of the $(E)$-enolate is also explained by Goodman et al. After (-)-Ipc are substituted for the methyl groups of the boron reagent in the calculation, the $(R e)$-facial attack of the aldehyde in the boat A TS of the $(E)$ enolate becomes favored but the chair one favors $(\mathrm{Si}$ )-facial attack, thus a poor enantioselective outcome of the aldol reaction is obtained (Figure 1.5).

Continuing from Paterson's study, ${ }^{32}$ Gennari and Paterson designed a new pair of enantiomeric boron reagents using menthol substituents, based on MM2 calculations of the cyclic TS of the aldol reaction. ${ }^{14,33,34}$ A number of ketones were enolized and interestingly, high preferences for $(E)$-enolates were observed. Furthermore, this chiral boron reagent is able to induce highly diastereo- and enantioinduction in the aldol reactions of $(E)$-enolates to form anti-aldol products. It was also discovered that this boron reagent exhibited a high stereoinduction in the aldol reactions of methyl thioesters with chiral $\alpha$-aminoaldehydes. With the different enantiomeric forms of the boron reagents (A and $\mathbf{B}$, Scheme 1.13) Gennari and Paterson were able to achieve both anti- and syn-aldol products in the aldol reactions of $\mathbf{C}$ and $\mathbf{D}$ (Scheme 1.13). ${ }^{14,33}$ It is worth pointing out that with chiral boron reagent $\mathbf{B}$, the inherent preference for the polar Felkin-Anh product is overcome, to afford the "mismatched" product with high stereoselectivity: the ratio of anti-Felkin:Felkin is 96.8:3.2, which is very close to $98.6: 1.4$ in the "matched" case. This result was an important step in the area of aldol chemistry, since achieving a good stereochemical outcomes from $(E)$-enolates and methyl enolates has proven very challenging in the past. ${ }^{6,14,35}$

Although the success of asymmetric aldol reactions of $(E)$ - and terminal enolates with the 

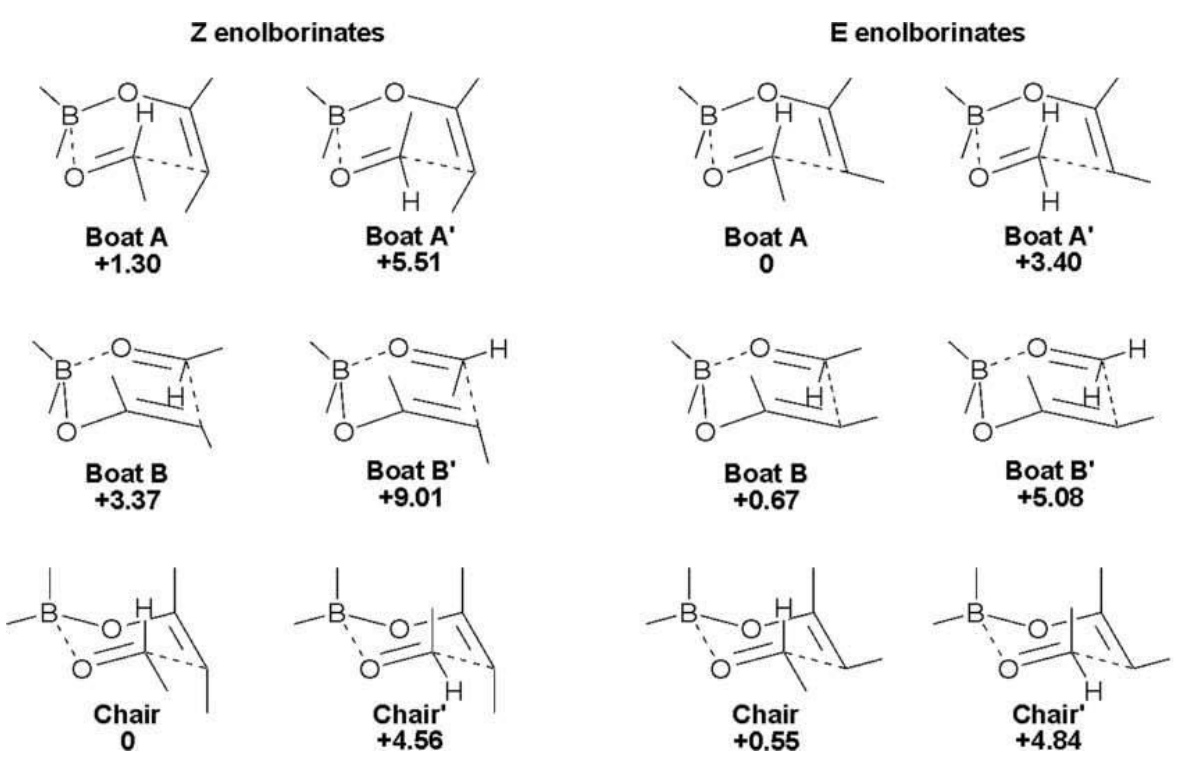

Note: Although Goodman's figure as reproduced shows a mixture of formaldehyde and acetaldehyde, all energies shown were calculated for TSs with acetaldehyde.

Figure 1.5: Goodman's Calculated Transition States of Butan-2-one Derived Enolates and Acetaldehydes (Relative Energies in kcal mol${ }^{-1}$, Reproduced from Goodman's Paper ${ }^{17}$ by Permission of The Royal Society of Chemistry)

menthol-derived chiral boron reagent solved many difficulties in aldol chemistry, the applications for methyl ketone derived enolates are still very limited. Nonetheless, the presence of a protected hydroxy group at the chiral $\beta$-carbon in an alkyl methyl ketone-derived enolate greatly favors the 1,5-anti-aldol product. The first example of a 1,5-anti-induction in an aldol reaction was demonstrated by Masamune's group in the synthesis of the $\mathrm{C}_{1}-\mathrm{C}_{16}$ fragment of bryostatin with methyl ketone $\mathbf{A}$ and aldehyde $\mathbf{B}$ with boron mediation (Table 1.3). ${ }^{35,36}$ With achiral diethylboron triflate, the protected $\beta$-hydroxy group can already give a weak anti-induction in the reaction (2:1 anti:syn) (Entry 1). The "matched" enantiomeric form of the boron reagent can improve the stereochemical outcome to 6:1 anti:syn (Entry 3). Later, Paterson and Evans ${ }^{37-39}$ extended this 1,5-anti selective aldol chemistry to different protected $\beta$-oxygenated alkyl methyl ketones (Scheme 1.14). The discovery of 1,5-anti induction in aldol reactions is very important for polyketide synthesis since the basic backbone of PKs contains 1,3-polyols which are well suited to formation by this chemistry.

\subsubsection{1,5-Anti Induction in Aldol Reaction}

\section{Evans-Paterson Aldol Reaction}

The stereocontrolled outcome of the Evans-Paterson aldol reaction depends heavily on the structure of the $\beta$-hydroxy protecting group. ${ }^{35,37-39}$ Excellent diastereomeric excesses in 1,5-anti aldol products are obtained when $\beta$-benzylic and benzylidene acetal protecting groups are attached to the methyl ketone (Reactions (1)-(3), Scheme 1.14). In contrast, a methyl ketone bearing a $\beta$-silyloxy protecting group is very poor (Reaction (4) and (5), Scheme 1.14) at directing 1,5-anti 


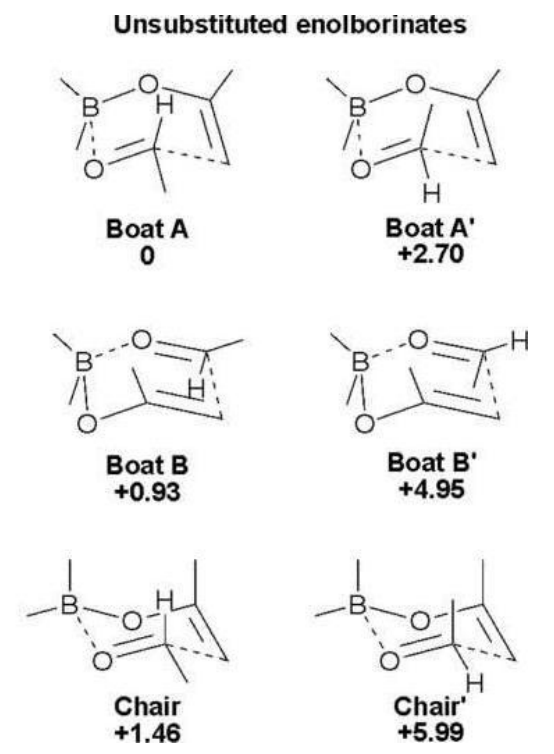

Figure 1.6: Goodman's Calculated Transition States of Acetone Derived Enolates and Acetaldehydes (Relative Energies in kcal mol ${ }^{-1}$, Reproduced from Goodman's Paper ${ }^{17}$ by Permission of The Royal Society of Chemistry)

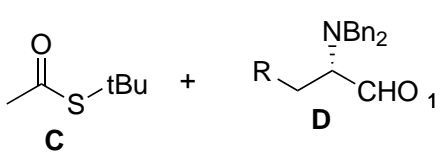

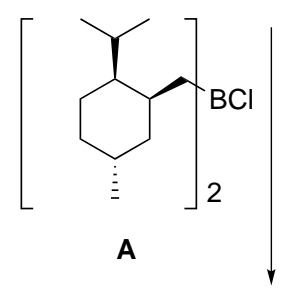<smiles>CC(C)CC(=O)CC(O)C(Br)Br</smiles>

(98.6:1.4 anti:syn)

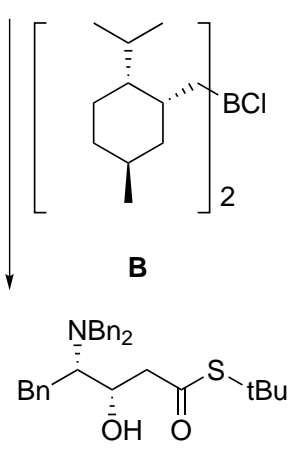

(96.8:3.2 anti:syn (anti-Felkin:Felkin))

Scheme 1.13: Menthol-derived Chiral Boron Chloride Directing Asymmetric Aldol Reactions of Methyl Ketone and $\alpha$-Amino Aldehyde ${ }^{14,34}$ 


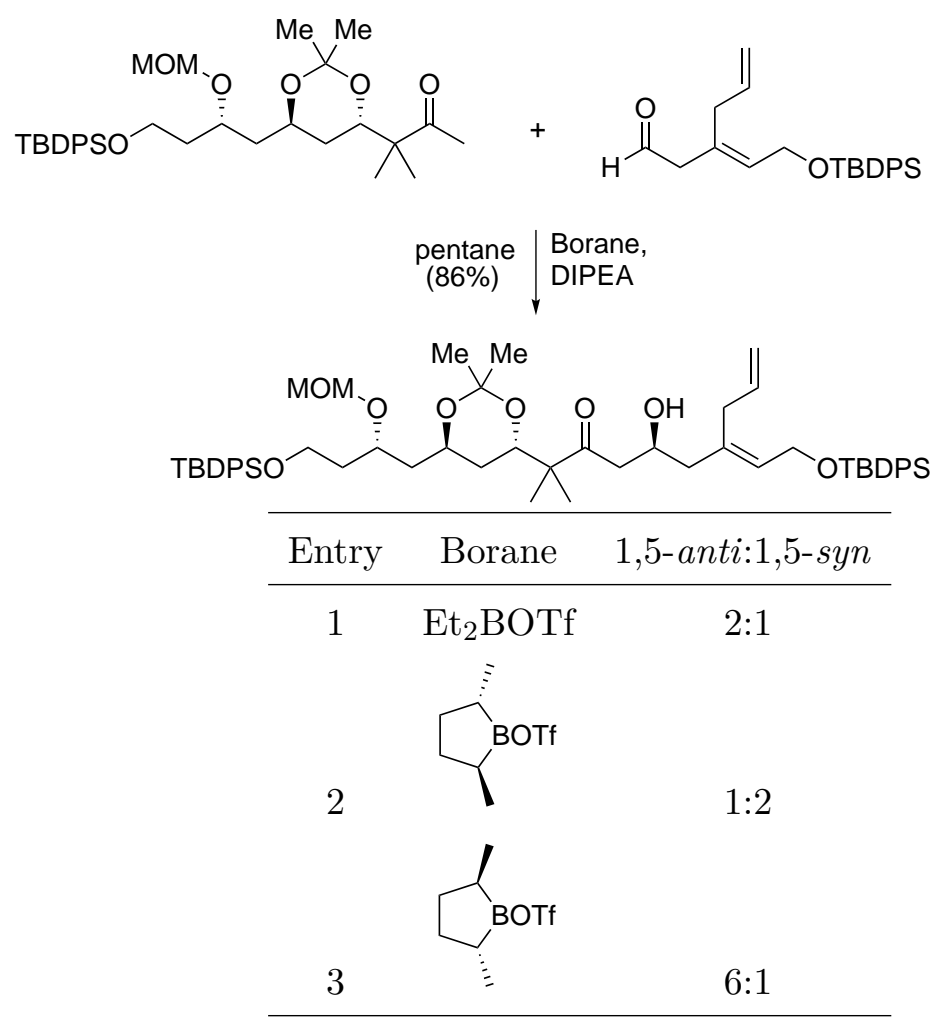

Table 1.3: Masamune's 1,5-Anti-Aldol Reactions for Coupling $\mathrm{C}_{10}-\mathrm{C}_{11}$ Bond of Bryostatin ${ }^{35,36}$

selectivity (Reaction (4) and (5), Scheme 1.14), except under special circumstances. ${ }^{4}$

\section{$\pi$-Stacking Model}

1,5-Anti-induction in an aldol reaction has been commonly used in natural product synthesis, including for peloruside A. ${ }^{3,35}$ The mechanism of Evans-Paterson aldol reactions was explained by Hoberg's group in their synthetic studies of peloruside A-like structures. ${ }^{4}$ They used a cyclic diphenylsilyl acetal protected alkyl methyl ketone $(R)-\mathbf{2}$ to afford moderate to good yields and excellent stereoselectivites in the aldol reaction that models the $\mathrm{C}_{11}-\mathrm{C}_{12}$ bond of peloruside $\mathrm{A}$ (Scheme 1.15).

However, on changing of the diphenyl to diisopropyl substituents on the silicon (from $\mathbf{2}$ to 4), this led to a dramatic decrease in stereoselectivity from $>99: 1$ to 1.2:1 (Reaction (2), Scheme 1.15). Furthermore, acyclic $(R)-\mathbf{5}$ bearing the phenyl substituents around the silicon of cyclic $(R)-2$ also gave poorly stereoselective aldol reactions (Reaction (4), Scheme 1.15). Hoberg et al. proposed a $\pi$-stacking model to explain these interesting observations and Evans' and Paterson's results (Figure 1.7). The model proposed that cyclic $(R)-2$ adopts a chair conformation in the TS, and one of the phenyl groups participates in a $\pi$-stacking interaction with the boron enolate to shield one face such that the aldehyde can only approach from the other one with the t-Bu group pointing away from the bulky aryl groups. When a single aryl group is present at the $\beta$-oxygen of the methyl ketone, molecular mechanics calculations confirm the ability to rotate to a conformation which can also undergo a $\pi$-stacking interaction. This model can explain the 1,5- 


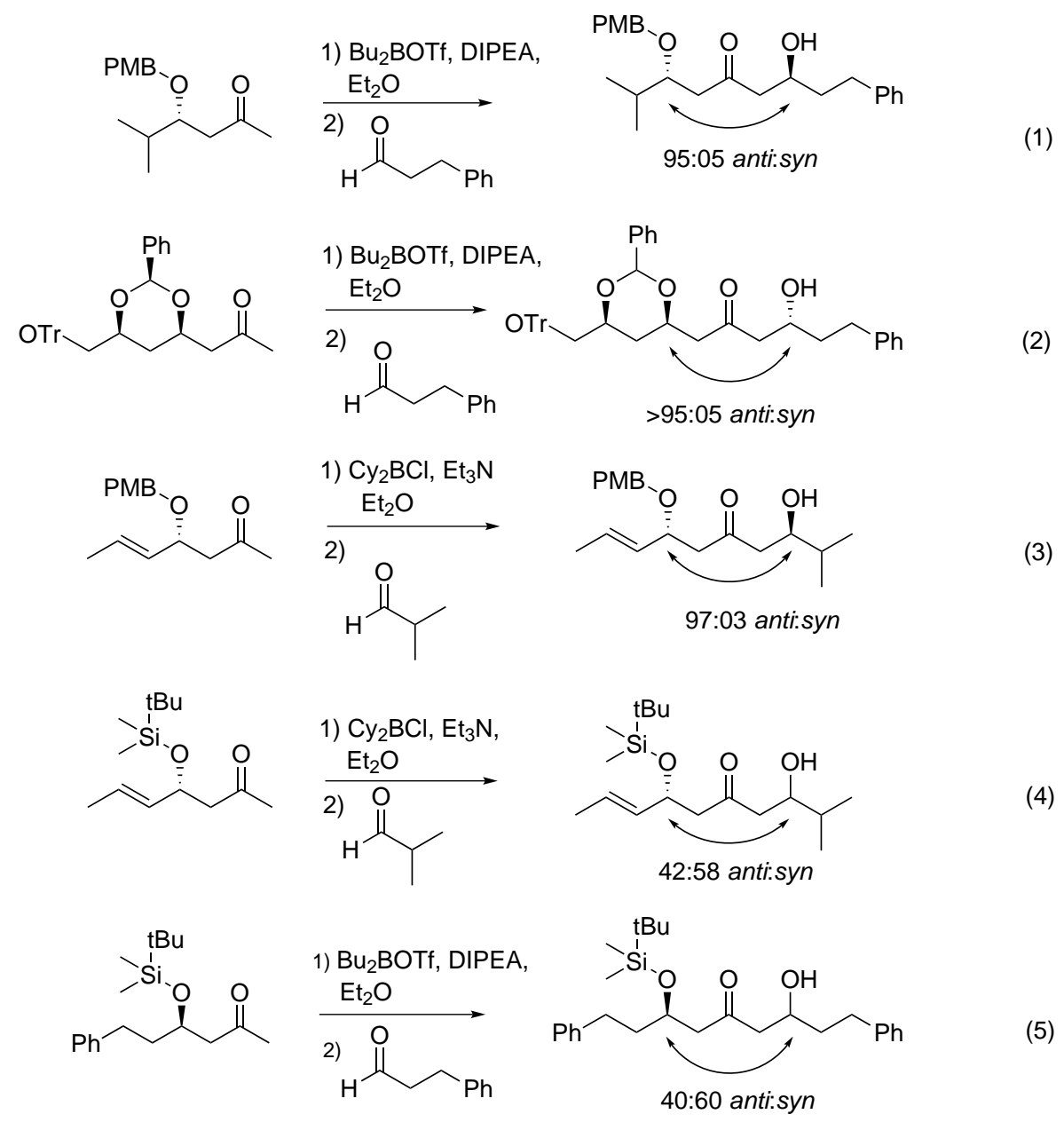

Scheme 1.14: 1,5-Anti Induction in Aldol Reaction with Different Protecting Groups ${ }^{37,38}$

anti directing effect from an aryl protecting group at the $\beta$-oxygen of the methyl ketone in the aldol reaction which accounts for most of Evans' and Paterson's results, however, it ignores the effect of the $\beta$-oxygen which does play a significant role in 1,5-anti induction, as demonstrated by the lack of stereoselectivity observed when $\beta-\mathrm{OCH}_{2} \mathrm{Ar}$ was changed to $-\mathrm{CH}_{2} \mathrm{Ar} .{ }^{6,37}$ Furthermore, a non-aryl group can also have a moderate directing effect for 1,5-anti-induction, as seen with the cyclic dimethyl acetal in Masamune's report (Table 1.3). ${ }^{36}$

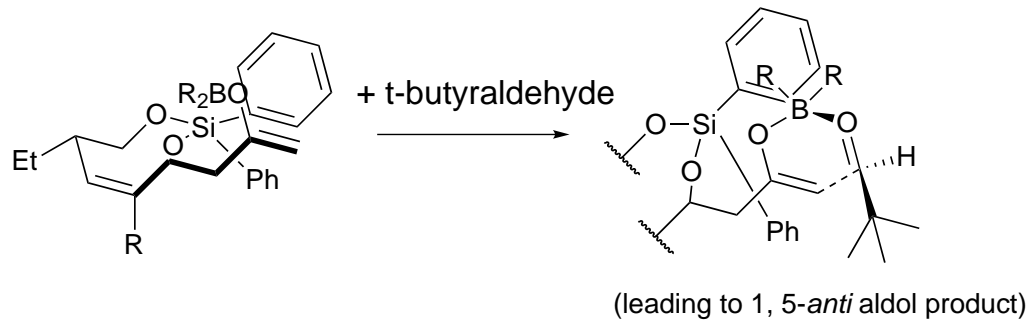

Figure 1.7: Hoberg's Model for 1,5-Anti-Induction in Aldol Reaction ${ }^{4,6}$ 


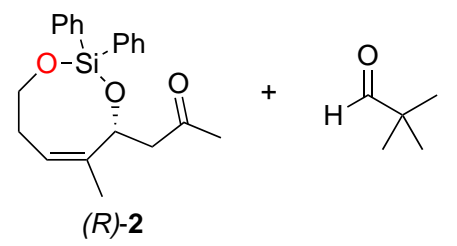<smiles>CC(=O)C[C@H]1O[Si](C(C)C)(C(C)C)OCCC=C1[18OH]</smiles>
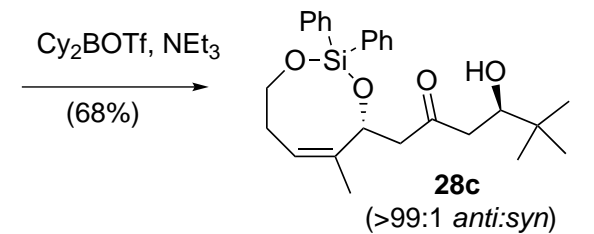

(1) ref. $4 \& 5$ (>99:1 anti:syn)
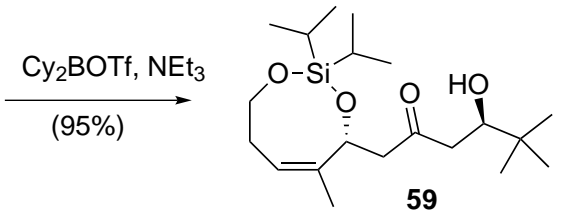

(1.2:1 anti:syn)<smiles>[R]C(CC(C)=O)O[Si](c1ccccc1)(c1ccccc1)c1ccccc1</smiles>

$(R)-5$

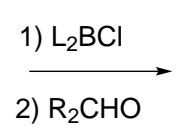

(2) ref. $4 \& 5$<smiles>[R]C(O)CC(=O)CC([R7])O[Si](C[R17]([H])([H])c1ccccc1)(c1ccccc1)c1ccccc1</smiles>

(3) ref. $4 \& 5$<smiles>[CH2+]C(=O)C[C@H]1O[Si](c2ccccc2)(c2ccccc2)OC[C@@H](CC)C=C1C</smiles>

(S)-6a

$$
\begin{array}{rrr} 
& \multicolumn{3}{c}{\text { anti }: \text { syn }} \\
& \mathrm{R}_{1}=\mathrm{iPr}, \mathrm{R}_{2}=\mathrm{iPr} & 2: 1,88 \% \text { yield } \\
& \mathrm{R}_{1}=\mathrm{iPr}, \mathrm{R}_{2}=\mathrm{tBu} & 2.5: 1,86 \% \text { yield } \\
\mathbf{4 0 a} & \mathrm{R}_{1}, \mathrm{R}_{2}= & 1: 1.2,89 \% \text { yield }
\end{array}
$$

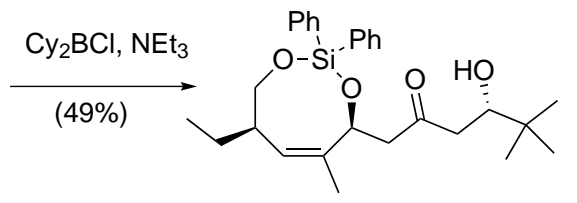

60

(>99:1 anti:syn)

Key:

a. Aldol product in this study is consistent with the expectations of Kishi's ${ }^{13} \mathrm{C}$ NMR database for 1,5-anti product.

Scheme 1.15: 1,5-Anti-Stereoinduction from Different Silyl Protected Methyl Ketones ${ }^{2,4,5}$

\section{Goodman's Model}

More recently, a computational study of the 1,5-anti aldol reaction was reported by Paton and Goodman. ${ }^{6}$ Analogous to calculated TSs for the aldol reaction of acetone with acetaldehyde (Figure 1.6 on page 23), the calculated boat TSs of a $\beta$-methoxy-substituted terminal enolate (Figure 1.8) are more stable than the chair ones (not shown). Surprisingly, the lower energy conformations are the In-Anti and the In-Syn boat conformers where the $\beta$-oxygen orients towards the six-membered ring rather than the ones with the group outwards (Out-Anti and Out-Syn boats). It was also noticed that the distance between the $\beta$-oxygen and the formyl hydrogen is remarkably short (2.322 $\AA$ In-Anti and $2.396 \AA$ In-Syn), thus Paton and Goodman postulated that this represented unusual hydrogen bonds formed in the In-Anti and In-Syn conformers to provide extra stabilization compared to the other two boat conformers. To generate the In-Anti and In-Syn boats in Figure 1.8, the alkyl chains have to fold in, which is sterically unfavorable, 
but the stabilizing $\mathrm{C}-\mathrm{H}--$ - O hydrogen bonds compensate for this and contribute 3.42 and $2.57 \mathrm{kcal} \mathrm{mol}^{-1}$ stabilization energies respectively (Figure 1.8). The terminal carbon chain of the enolate in the In-Syn boat would interact adversely with the substituent of the boron reagent, thus leading to a weaker hydrogen bond $\left(2.57 \mathrm{kcal} \mathrm{mol}^{-1}\right)$ and a less stable TS. In contrast, the $\beta$-hydrogen of the enolate points to the boron ligands in the In-Anti boat, which minimizes the steric interference. Therefore, the 1,5-anti aldol product is generated preferentially from the In-Anti TS. Very similar observations are obtained in the calculated TSs of $\beta$-benzylic ether- and $\beta$-benzylidene acetal-substituted terminal enolates, indicating that Goodman's hydrogen bondstabilized In-Anti TS has general application to the 1,5-anti aldol reaction. Generic Goodman's anti- and syn-TSs are shown in Figure 1.9.

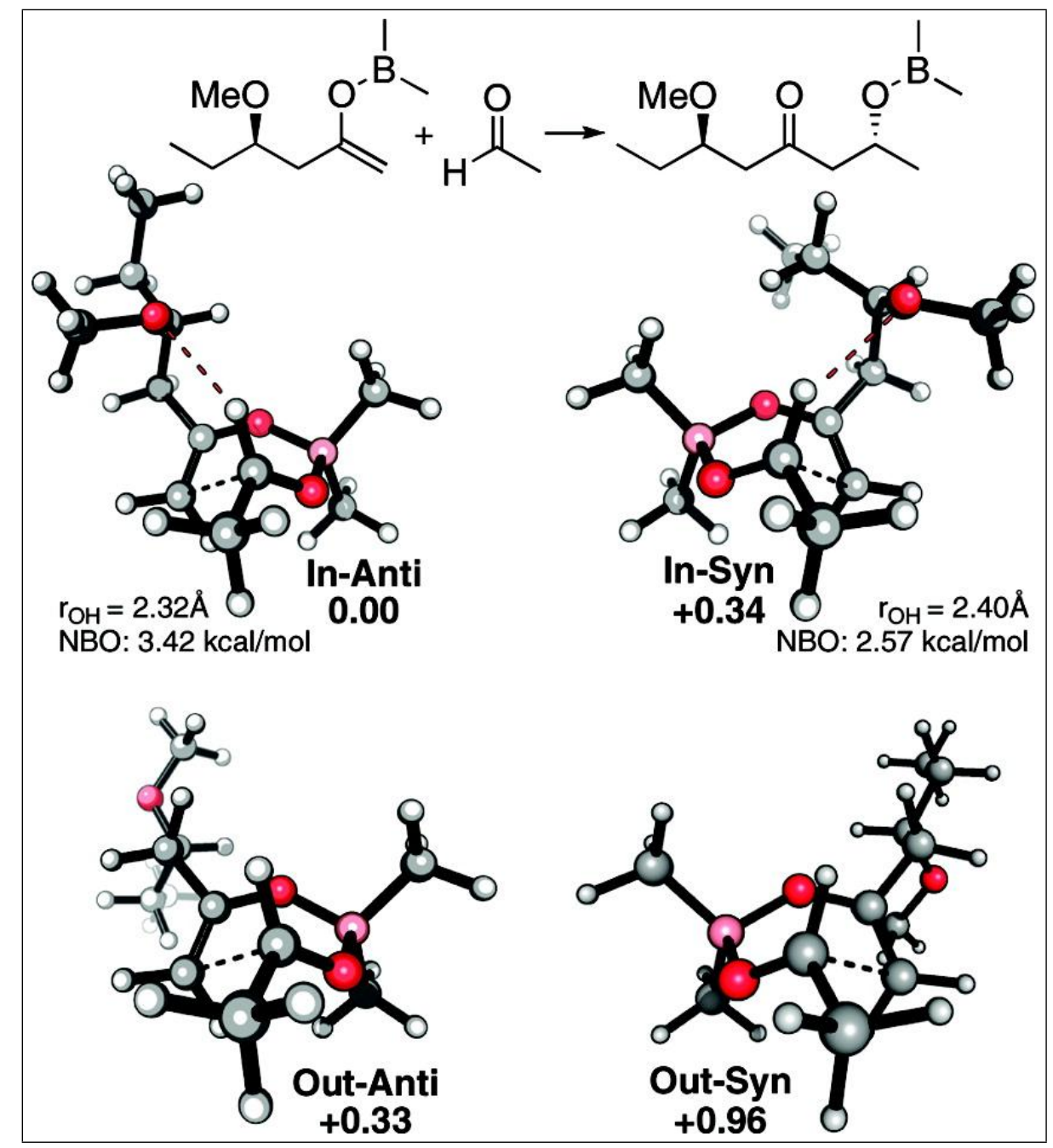

Red sphere $=$ oxygen, pink sphere $=$ boron, grey sphere $=$ carbon, white sphere $=$ hydrogen .

Figure 1.8: Goodman's Model for 1,5-anti-Induction in Aldol Reaction (Reproduced from Goodman's Paper ${ }^{6}$ by Permission of The American Chemical Society)

Goodman et al. also explained why the stereoinduction is low when the $\beta$-oxygen is protected as a silyl ether group. They noticed that when the $\beta$-hydroxy protecting group was 


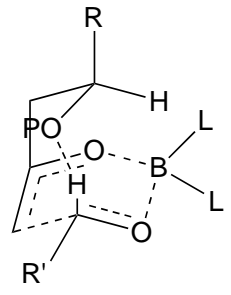

anti

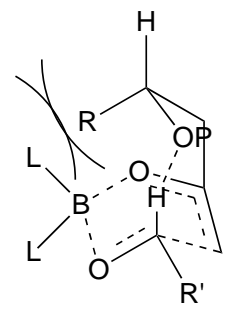

syn

Figure 1.9: Generic Goodman's Transition States

TMS- or TBDMS-, there are two low energy boat TSs: Out-Anti and In-Syn (Figure 1.10). This change is presumably caused by the increased bond length of the $\mathrm{C}-\mathrm{H}$ - - - O hydrogen bond due to the steric influence of the silyl protecting group which was considerably higher than for benzyl or benzylidene acetal groups. According to Goodman's data, the calculated $\mathrm{C}-\mathrm{H}$ - - - O delocalization energy was only $1.46 \mathrm{kcal} \mathrm{mol}^{-1}$ for the In-Anti TS of a TMS-protected $\beta$-oxygenated methyl ketone compared to $3.42,3.29$ and $4.07 \mathrm{kcal} \mathrm{mol}^{-1}$ for those of methoxy, benzyl and benzylidene acetal protecting groups, respectively (Figure 1.10). Furthermore, the empty $d$-orbital of the silicon was observed to significantly interact with the electrons on the oxygen in the TSs, therefore, the oxygen became electron deficient and a weaker "hydrogen bond" was formed in In-Anti and In-Syn TSs. ${ }^{6}$

Goodman's hydrogen bonding model has a broader coverage than Hoberg's $\pi$-stacking model and also explains why low stereoselectivities were observed with $\beta$-alkyl and silyloxy directing groups. However, it does not explain why there is a striking difference in 1,5-anti directing effect between the diisopropylsilyl acetals, diphenylsilyl acetal, and $t$-butyldiphenylsilylether group (Scheme 1.15) in Hoberg's work ${ }^{4}$ and how the diphenylsilyl acetal directs the excellent stereoselective aldol reaction. In Turner's $\mathrm{PhD}$ thesis, ${ }^{2}$ an attempt to use Goodman's model to explain Hoberg's observations was outlined. It was discovered that the presence of another chiral center in the eight-membered silyl acetal of $\mathbf{6 a}$ did not affect its excellent stereoinduction (Reaction (4), Scheme 1.15). Combining this with Hoberg's results (Reactions (1), (2), (3) and (4), Scheme 1.15), led to the postulate that the $\pi$-electrons from the phenyl rings of $\mathbf{2}$ and $\mathbf{6 a}$ interacted with the empty $d$-orbital of the silicon to diminish the electron withdrawing effect such that the $\beta$-oxygen was able to form a formyl hydrogen bond, leading to the stable 1,5-anti Goodman TS (Figure 1.7). In comparison, the alkyl groups of the diisopropylsilyl acetal did not stabilize the silicon so that no strong Goodman hydrogen bond was formed in the TS. However, Turner did not explain how the corresponding In-Anti boat TS of $\mathbf{2}$ compensates for the steric bulk of the diphenylsilyl acetal group which is much bulkier than TMSO group included in Goodman's calculation. 


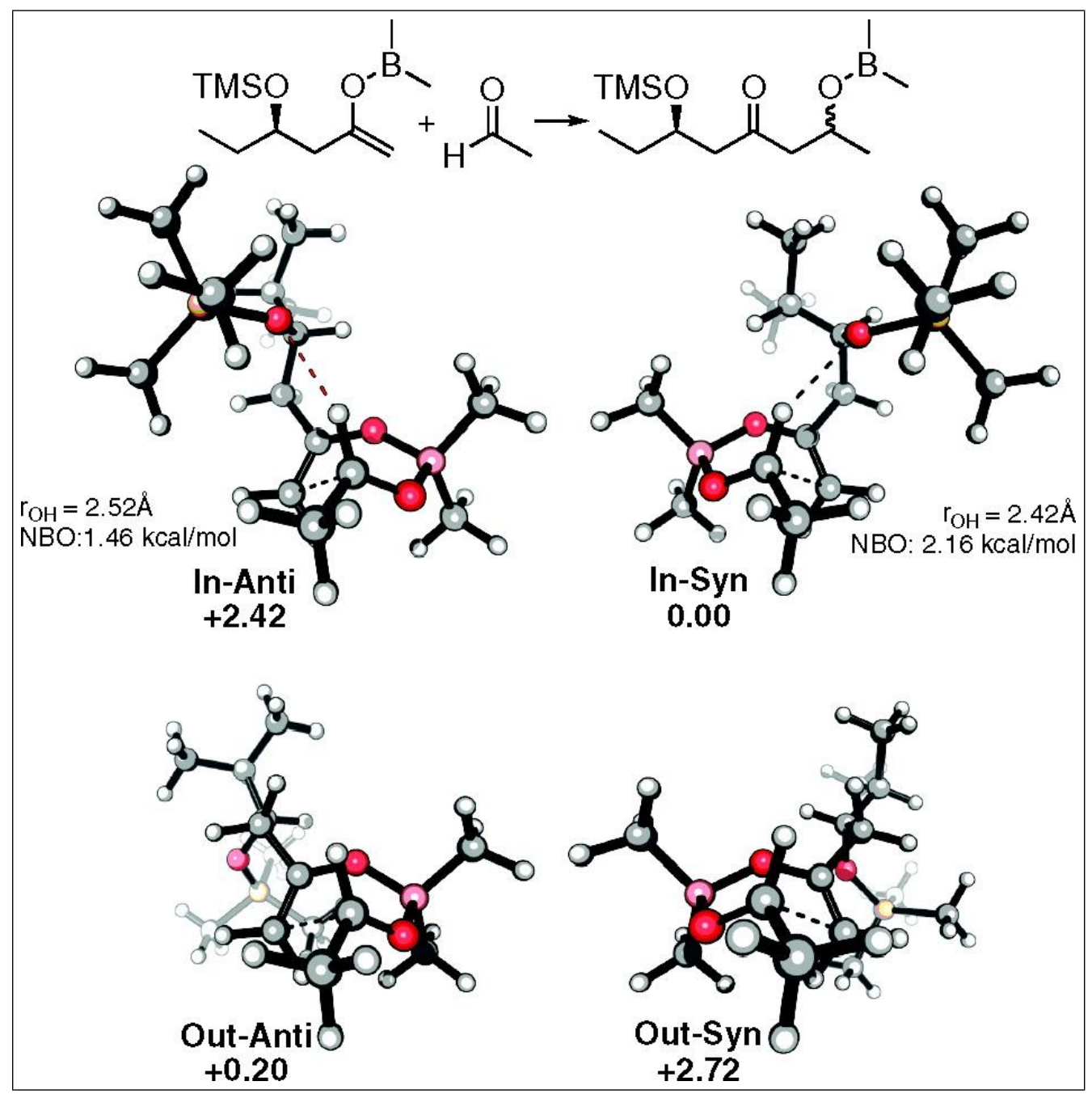

Red sphere $=$ oxygen, pink sphere $=$ boron, grey sphere $=$ carbon, white sphere $=$ hydrogen, yellow sphere $=$ silicon.

Figure 1.10: Goodman's Model for $\beta$-siloxy Directing 1,5-Anti-Induction (Reproduced from Goodman's Paper ${ }^{6}$ by Permission of The American Chemical Society)

\section{Abiko's Diborylated Enolates}

Carboxylic esters are typically inactive under most enolization conditions and it was reported that the enolization of methyl propionate was unsuccessful with a boron triflate and an amine. ${ }^{40}$ Thus, the aldol reaction of esters using boron reagents remained unexplored until 2002, when Abiko's group reported a new procedure for preparation of a dialkylboron triflate reagent and the use of this boron reagent to successfully mediate aldol reactions of esters that contain a sulfonamide-type chiral auxiliaries. ${ }^{7,40,41}$ Surprisingly, bis-aldols are produced in the boronmediated aldol reactions of acetate esters. ${ }^{7,41}$ Furthermore, the chiral auxiliary-bound acetate $\mathbf{7}$ designed by Abiko's group produced the bis-aldol product (8a) with high stereoselectivity (Reaction (1) in Scheme 1.16). The underlying mechanism of the double aldol reaction was also investigated using variable temperature NMR experiments on a simple system involving methyl 
acetate with $\mathrm{Cy}_{2} \mathrm{BCl}$ and $\mathrm{Et}_{3} \mathrm{~N}$ by Abiko's group. ${ }^{7,41}$ When 1.0 eq. methyl acetate was mixed with 2.5 eq. $\mathrm{Cy}_{2} \mathrm{BOTf}$ in $\mathrm{CDCl}_{3}$ (concentration $=0.27 \mathrm{M}$ for methyl acetate), a downfield shift of the protons of methyl acetate was observed in the ${ }^{1} \mathrm{H}$ NMR spectrum, indicating a chelation of methyl acetate to the boron reagent. Surprisingly, the addition of $\mathrm{Et}_{3} \mathrm{~N}$ (3.0 eq.) provided a (Z)-diborylated enolate 9 exclusively (Reaction (2), Scheme 1.16). The structure of this doubly borylated enolate was deduced using a variety of NMR experiments as explained in the following content. ${ }^{41}$ The ${ }^{1} \mathrm{H}$ NMR showed the following changes, indicating the transformation to a keteneacetal: 1) an -0.10 ppm upfield shift of the methoxy group, 2) the appearance of a new signal at $4.35 \mathrm{ppm}$ (intergrated as $1 \mathrm{H}$ ) accompanied to the vanishing acetyl methyl signal at $2.15 \mathrm{ppm}$. The ${ }^{13} \mathrm{C}$ NMR also showed two new peaks at 169.9 and $79.5 \mathrm{ppm}$ corresponding the two carbons of the keteneacetal. The COSY and the DEPT spectra confirmed the assignments of the teriminal olefinic carbon $(79.5 \mathrm{ppm})$ and proton $(4.35 \mathrm{ppm})$, the methoxy group $\left({ }^{1} \mathrm{H}, 3.65\right.$ ppm; $\left.{ }^{13} \mathrm{C}, 55.3 \mathrm{ppm}\right)$, and the methine groups of the cyclohexyl substituents $\left({ }^{1} \mathrm{H}, 1.1\right.$ and 1.6 ppm; ${ }^{13} \mathrm{C}, 29.8$ and $33.3 \mathrm{ppm}$ ). The HMBC (delay time $65 \mathrm{~ms}$ ) revealed the correlation between the methine proton of a cyclohexyl group $\left({ }^{1} \mathrm{H}, 1.6 \mathrm{ppm}\right)$ and the terminal olefinic carbon $(79.5$ ppm), and as expected, the correlation between the olefinic proton (4.35 ppm) and the methine carbon $(33.3 \mathrm{ppm})$. In addition, upon decoupling ${ }^{11} \mathrm{~B}$, the intensity of the cross-peak of the olefinic carbon $(79.5 \mathrm{ppm})$ and proton $(4.35 \mathrm{ppm})$ increased 3.7 times due to the decrease of the quadrupolar effect of ${ }^{11} \mathrm{~B}$ nuclei on the olefinic carbon. These observations indicated the formation of a carbon and oxygen-bound diborylated enolate in the enolization. ${ }^{41}$ The immediate work-up step with $\mathrm{D}_{2} \mathrm{O}$ and $\mathrm{CD}_{3} \mathrm{OD}$, from which the dideuterated material was isolated (Reaction (2), Scheme 1.16), also confirmed this structure. ${ }^{7}$ The stereochemistry of this $(Z)$ diborylated enolate was determined with a NOESY experiment in which a correlation between the methoxy group and the olefinic proton was observed. ${ }^{41}$

Similar NMR experiments were also conducted with the chiral auxiliary-bound 7 (Scheme $1.17)$ and the corresponding diborylated enolates $\mathbf{1 0}$ were also detected but both $(E)$ - and $(Z)$ 10 were present. The order of adding $\mathrm{Cy}_{2} \mathrm{BCl}, \mathrm{Et}_{3} \mathrm{~N}$ and ketones (methyl acetate and 7) in these reactions did not affect the stereochemical outcome of the enolization. However, it was found that the temperature for the aldol reaction of $\mathbf{7}$ altered the stereoselectivity as shown in Scheme 1.17. When the enolization was performed at $-73{ }^{\circ} \mathrm{C},(E)-\mathbf{1 0}$ was favored with $>95: 5$ $(E):(Z)$ preference but the ratio decreased as the temperature was raised and $(Z)$-10 became dominant $(1: 3(E):(Z))$ at $-3{ }^{\circ} \mathrm{C}$. The ratio between these two enolates directly correlated with the diastereomeric ratio $(\mathrm{dr})$ of the subsequent bis-aldol reaction with isobutyraldehyde. For example, the ratio of $(E)-\mathbf{1 0}:(Z)-\mathbf{1 0}$ was $1: 3$ at $-3{ }^{\circ} \mathrm{C}$ similar to the ratio of $\mathbf{8 a}: \mathbf{8 b}(23: 67)$ obtained after the addition of isobutyraldehyde at $0{ }^{\circ} \mathrm{C}$. This result indicates that the bis-aldols generated in the boron-mediated aldol reaction of alkyl acetates are due to the formation of the diborylated enolates when excess amounts of a boron reagent (2.5 eq.) and an amine (3.0 eq.) are used. 

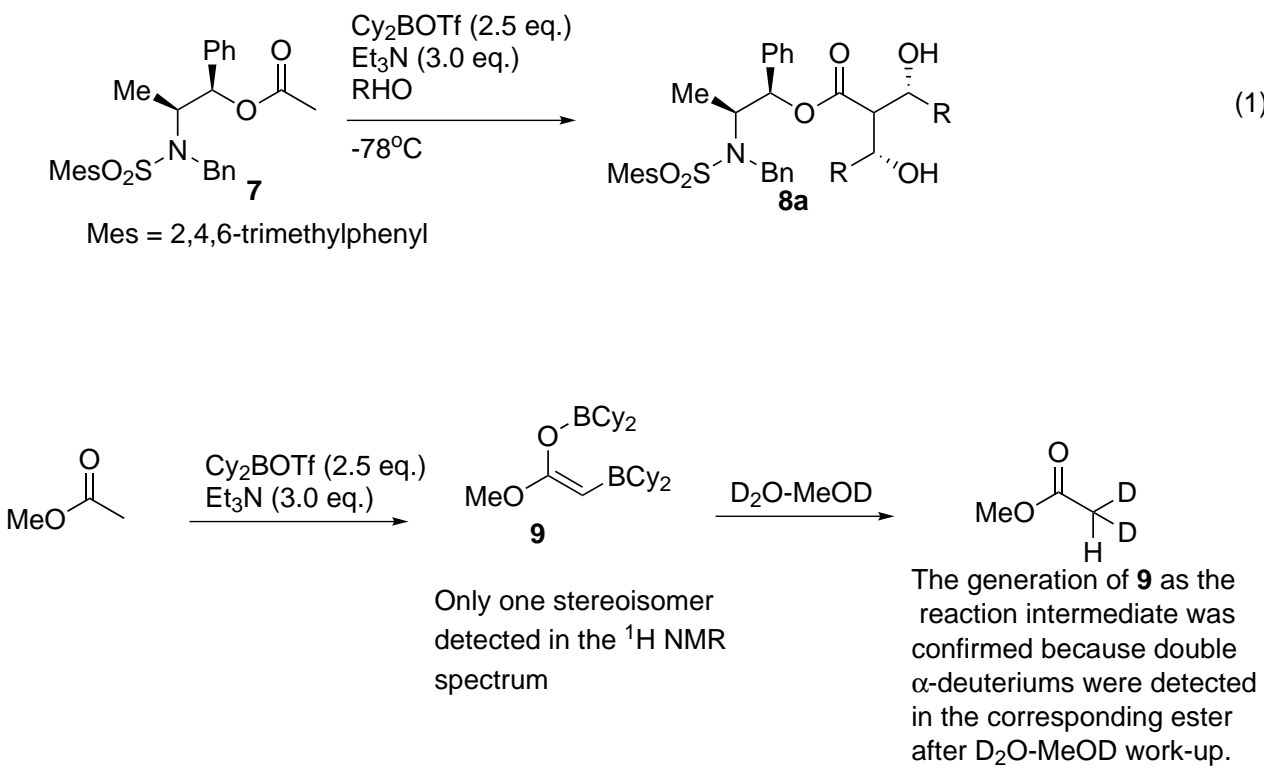

Scheme 1.16: Bis-Aldol Reactions of $\mathbf{7}$ and Evidence for the Formation of Doubly Borylated Enolate 9

Doubly borylated enolates were formed with all of the alkyl acetates used in Abiko's study ${ }^{7}$ and certain types of thioesters and ketones under certain conditions provided doubly borylated enolates as shown in Table 1.4. For example, only oxygen bound mono-boron enolates were detected from aliphatic ketones, such as 2-butanone and acetophenone (Entry 6 and 7). In contrast, methoxyacetone favored the formation of a mono-O-boron enolate (>98\%) using 1.0 eq. $\mathrm{Cy}_{2} \mathrm{BCl}$ and 1.3 eq. $\mathrm{Et}_{3} \mathrm{~N}$ (Entry 8 ) but a diborylated enolate became dominant (>98\%) when excess amount of $\mathrm{Cy}_{2} \mathrm{BCl}$ (2.5 eq.) and $\mathrm{Et}_{3} \mathrm{~N}$ (3.0 eq.) were used (Entry 9). These results indicate that an oxygen-bound mono-enolate is formed first in the enolization of a ketone but the presence of a nearby electron donating functional group in the ketone, such as an $\alpha$-methoxy group, may allow the formation of diborylated enolate, as proposed by Abiko et al. ${ }^{7}$ 


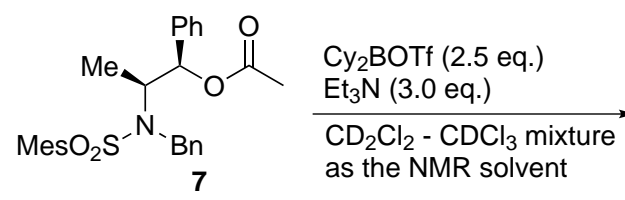

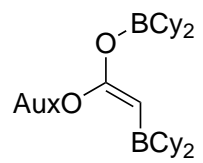

(E)-10

at $-23^{\circ} \mathrm{C} \quad 1$

at $-3^{\circ} \mathrm{C} \quad 1$

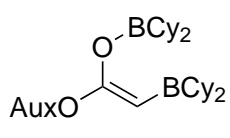

(Z)-10
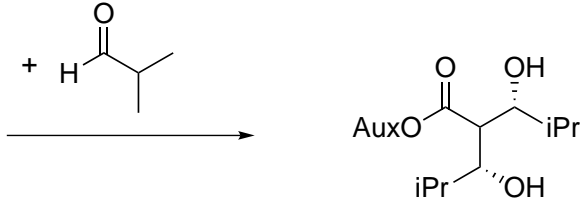

8 a at $-78^{\circ} \mathrm{C} \quad 88$<smiles>[TeH4]=[W]</smiles>
at $0^{\circ} \mathrm{C} \quad 23$ $\mathrm{MesO}_{2} \mathrm{~S}^{-\mathrm{N}_{-}} \mathrm{Bn}$

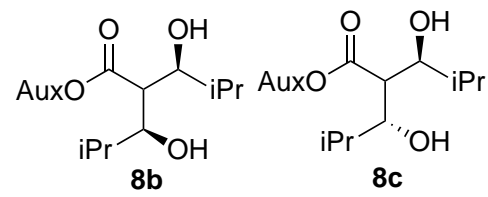



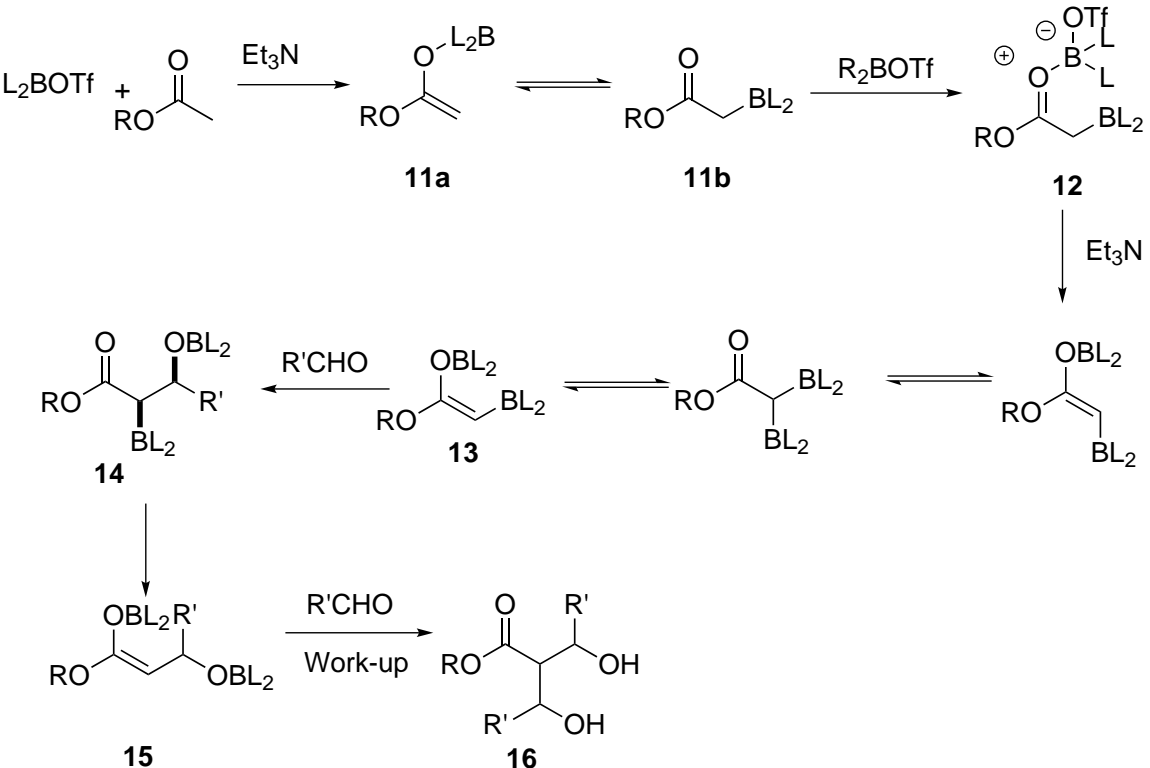

Key:

$L=$ alkyl substituent

Scheme 1.18: Proposed Mechanism for Bis-Aldol Reaction of Acetate Ester Proposed by Abiko et al. ${ }^{7}$

A mechanism for the bis-aldol reaction was also proposed by Abiko's group (Scheme 1.18). ${ }^{7}$ An oxygen bound mono-boron enolate 11a is thought to form first and interconverts with an $\alpha$-boron substituted ester $\mathbf{1 1} \mathbf{b}$ by rearrangement. The carbonyl oxygen of $\mathbf{1 1} \mathbf{b}$ can attack a second boron triflate to form a diborylated ester 12, which is enolized again, followed by a rearrangement to form the $(\mathrm{Z})$-diborylated enolate 13. The aldol reaction of the $(Z)$-diborylated enolate with an aldehyde presumably goes through a Zimmerman-Traxler TS to form 14. Another rearrangement occurs to convert 14 to the corresponding diborylated enolate $\mathbf{1 5}$ which undergoes a second aldol reaction to form bis-aldol product $\mathbf{1 6}$.

The results reported by Abiko's group contribute greatly to the understanding and application of boron-mediated aldol reactions since they comprise 1) a new method for the synthesis of boron triflates, 2) a method for enolizing poorly active esters, 3) detection of aldol reaction intermediates with ${ }^{1} \mathrm{H}$ and ${ }^{13} \mathrm{C}$ NMR and 4) the discovery of doubly borylated enolates which are responsible for the formation of bis-aldol products. The doubly borylated enolates possibly represent important reaction intermediates in boron-mediated aldol reactions. Therefore, $\beta$-oxygenated alkyl methyl ketones could generate diborylated enolates which could participate in Zimmerman-Traxler TSs with aldehydes and leading to 1,5-anti aldol products. Abiko's method of preparing $\mathrm{Cy}_{2}$ BOTf was attempted in our study and a modification of Abiko's NMR experiment was also used in the investigations of the stereoinducing mechanisms of $\mathbf{2}$ and $\mathbf{3}$. 


\section{Chapter 2}

\section{Objectives of the Research}

By using a structural motif relevant to the fragments used in syntheses of peloruside $\mathrm{A}$ and its analogues, the project aim was to further improve the understanding of the 1,5-anti aldol reaction.

Specific objectives are:

1. To reveal the relative stereochemistry of the product from the aldol reaction of $\mathbf{2}$ by preparing a C-2 symmetric compound as the NMR reference and using Kishi's ${ }^{13} \mathrm{C}$ NMR database (page 48).

2. To investigate the effect of different silyl protecting groups on steric, energetic and entropic factors in aldol reactions of protected $\beta$-oxygenated alkyl methyl ketones.

3. To reveal the preferred conformation of the transition state of the aldol reaction of $\mathbf{2}$ with the help of computational modelling and high field NMR experiments.

4. To use methyl ketones $\mathbf{2}$ and $\mathbf{3}$ to investigate the impact of different $\alpha$ - and $\beta$-substituent(s) and electronic factors on the aldehydes. 


\section{Chapter 3}

\section{Results and Discussion}

To achieve Objectives 1 and 2 described in Chapter 2, it was necessary to synthesize a series of protected $\beta$-oxygenated methyl ketones in both enantiomeric forms and as racemates. To do so, syntheses of various chiral and achiral boron reagents were required. These are discussed in Section 3.1. The syntheses of unprotected $\beta$-hydroxy alkyl methyl ketones are discussed in Section 3.2. The syntheses of various protected $\beta$-oxygenated ketones and their aldol reactions were discussed in Sections 3.3 and 3.4. A number of methods used in determining the relative stereochemistry of the aldol products are also discussed.

Because the results described in Section 3.4 conflict with Goodman's model of 1,5-antistereoinduction (page 26), Objective 3 was pursued and a NMR study of various aldol reactions was carried out. Preferred conformations of the eight-membered ring of $\mathbf{2}$ was also investigated in this study with the help of computational modelling, as discussed in Section 3.5.

To attain Objective 4, a variety of aldol reactions of $\mathbf{2}$ and $\mathbf{3}$ with various aldehydes bearing groups having different steric and electronic properties were investigated, as summarized in Section 3.6.

\subsection{Synthesis of Dialkylboron Triflate and Chloride Reagents}

Boron mediation has been found to effectively produce 1,5-anti induction in certain aldol reactions of $\beta$-oxygenated methyl ketones. ${ }^{6,35}$ However, most boron mediating agents, such as dialkylboron triflates and monoalkylboron ditriflates, are heat and moisture sensitive. ${ }^{32}$ After a few failed trials of aldol reactions of acetone with methacrolein using commercial bottles of dibutylboron triflate (see Entry 1, Table 3.1 on page 38), we decided to synthesize different boron triflate and chloride reagents, and used the aldol reaction of acetone with methacrolein to evaluate their quality. (+)-(Ipc) ${ }_{2}$ BOTf was synthesized first according to Paterson's procedure (Reaction (1), Scheme 3.1). ${ }^{32}$ The boron triflate reagent (+)-17a was prepared in hexane by adding triflic acid to a suspension of $(+)-(\mathrm{Ipc})_{2} \mathrm{BH}$ in dry hexane at $0{ }^{\circ} \mathrm{C}$ and used directly in 
the following aldol reaction. However, the reaction afforded the aldol product $(S)-\mathbf{1 8}$ in only $44.2 \%$ yield (Entry 2, Table 3.1 ) which is significantly less than $59 \%$ reported in the literature. ${ }^{32}$ Although the concentration of the boron triflate reagent is worked out (approx. 1.9M) and reported in Paterson's procedure, the quality of the reagent can only be maintained in hexane for 24 h. ${ }^{32}$ Therefore, we decided to purify this triflate reagent so that the quality can be maintained and the quantity of $(+)-(\mathrm{Ipc})_{2}$ BOTf can be added accurately. However, several attempts at isolating this reagent out of the hexane solution were not successful, including rotatory evaporation of hexane under inert atmosphere, removing hexane under high vacuum (0-1 mbar) and recrystallization, and large amounts of an unreactive white solid were isolated instead, presumably due to the reaction of $(+)-(\mathrm{Ipc})_{2} \mathrm{BOTf}$ with moisture to form $(+)-(\mathrm{Ipc})_{2} \mathrm{BOH}$. Alternatively, the synthesis of $\mathrm{Cy}_{2} \mathrm{BOTf} \mathbf{1 7} \mathbf{b}$ was tried according to Abiko's procedure ${ }^{40}$ and recrystallization was used in the purification step (Reaction (2), Scheme 3.1). However recrystallizing this triflate reagent in hexane at $-20{ }^{\circ} \mathrm{C}$ was not successful, further lowering the mixture to $-78{ }^{\circ} \mathrm{C}$ afforded an unknown semi-solid that was not active in the aldol reaction (Entry 3, Table 3.1).<smiles>[X]C(C)[C@H]1CC[C@H](C)[C@H]2CC[C@H]1C2</smiles>

1) $\mathrm{BH}_{3} \cdot \mathrm{SMe}_{2}$

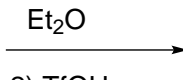

2) $\mathrm{TfOH}$

( $60 \%$ conversion assumed)

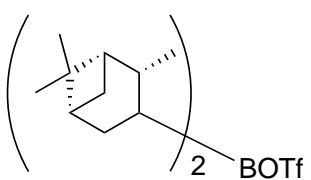

$(+)-(\mathrm{Ipc})_{2} \mathrm{BOTf}$

$(+)-17 a$ in a solution of hexane

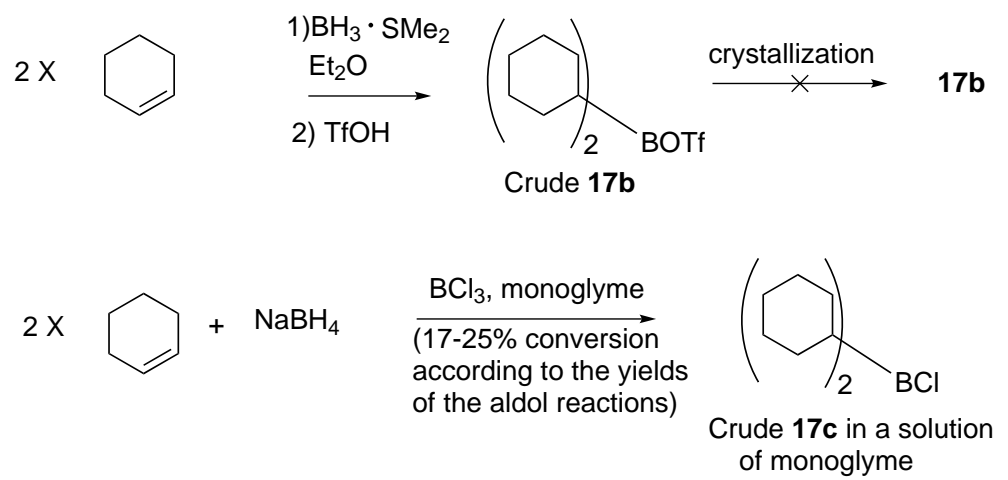

Scheme 3.1: Syntheses of Boron Triflate and Chloride Reagents using Paterson's, Abiko's and Reider's Methods ${ }^{32}$

In general, the boron triflate reagents were sensitive to oxidation and moisture. This may explain the poor reactivity in the use of commercial dibutylboron triflate (Entry 1, Table 3.1). We also attempted to synthesize more stable dialkylboron chlorides. Most attempts to prepare neat or a solution of $\mathrm{Cy}_{2} \mathrm{BCl} \mathbf{1 7} \mathbf{c}$ were unsuccessful. The best yield was afforded with the reaction of cyclohexene with sodium boron hydride and boron trichloride, as reported by Reider's group (Reaction (3), Scheme 3.1). ${ }^{42}$ As shown in Entry 4, Table 3.1, the yield of the aldol reaction of acetone and methacrolein using this boron reagent was only $17-25 \%$, which is significantly lower than that with commercial $\mathrm{Cy}_{2} \mathrm{BCl}\left(\sim 40 \%\right.$, Entry 5, Table 3.1). Since commercial $\mathrm{Cy}_{2} \mathrm{BCl}$ had 
a better reactivity than the prepared $\mathrm{Cy}_{2} \mathrm{BCl}$, it was used in the following boron-mediated aldol reactions, except in the synthesis of $(S)-\mathbf{1 8}$.

\subsection{Syntheses of $\beta$-Hydroxy Methyl Ketones}

To achieve the four objectives described in Chapter 2 on page 34, it is necessary to prepare significant quantities of $(S)-,(R)$ - and (rac)-18 methyl ketones.

\subsubsection{Paterson's Asymmetric Aldol Reaction and Synthesis of $(S)-18$}

An "acetate-type" aldol reaction was used in the asymmetric synthesis of $\mathbf{1 8}$, for which the attainment of high stereoselectivity is typically challenging. As described in Section 1.4.1 (page 18), moderate to low stereoinductions with (+)- and (-)-(Ipc) $)_{2}$ BOTf happen in most acetatetype aldol reactions (Table 1.2 on page 20) but a good enantioselectivity was achieved in the synthesis of $(S)$-18 with $(+)-(\operatorname{Ipc})_{2}$ BOTf (lit. $73 \%$ ee), as reported by Paterson et al. ${ }^{32}$

As described in Section 3.1, the synthesis of $(S)$-18 following Paterson's procedure using in situ prepared (+)-(Ipc) ${ }_{2}$ BOTf solution $((+)-\mathbf{1 7 a})$ afforded a lower yield $(44 \%)$ compared to $59 \%$ reported in the literature (Reaction (1), Scheme 3.2 and Entry 2, Table 3.1). Performing flash chromatography twice was required to purify the $(S) \mathbf{- 1 8}$ since the by-product, $(+)-\mathrm{IpcOH}$, had a similar mobility to the product in the chromatography. Determination of the ee of $(S) \mathbf{- 1 8}$ was attempted with Mosher ester by Tho previously, ${ }^{44}$ however the resulting product decomposed rapidly, possibly because the $\beta$-Mosher ester was a good leaving group and an E1cB elimination could happen (Reaction (1), Scheme 3.3). Thus the ee of $(S) \mathbf{- 1 8}$ was not determined but $73 \%$ ee was reported in the literature. ${ }^{32}$ Because of the tedious steps for preparing (-)-(Ipc) ${ }_{2}$ BOTf, $(R)$-18 was synthesized using a different method.

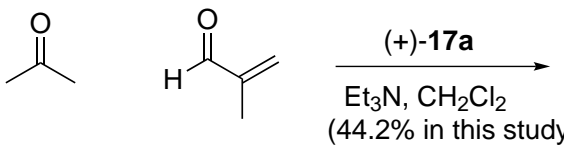

$(44.2 \%$ in this study)<smiles>CC(C)=O</smiles><smiles>C=C(C)C=O</smiles>
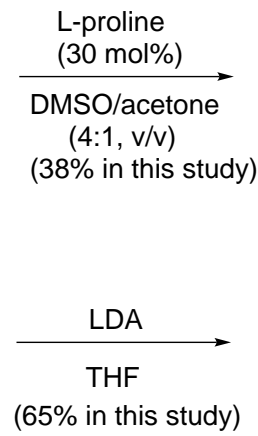<smiles>C=C(C)C(O)CC(C)=O</smiles>

(S)-18 (lit. 73\% ee, ref. 19)<smiles>C=C(C)[C@H](O)CC(C)=O</smiles>

(R)-18 (lit. 74\% ee, ref. 24)<smiles>C=C(C)[C@@H](O)CC(C)=O</smiles>

(rac)-18 (ref. 2)

Scheme 3.2: Acetone Aldol Reactions Employed in the Syntheses of (S)-, (R) and (rac)-18 $\mathbf{1 8}^{2,32,43}$ 


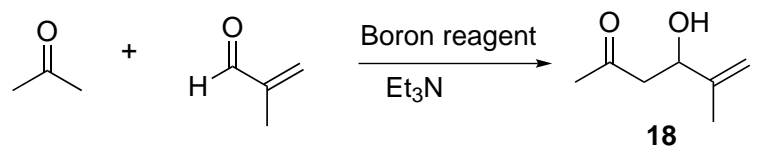

\begin{tabular}{|c|c|l|l|l|}
\hline Entry & Boron reagent & $\begin{array}{l}\text { Preparation } \\
\text { procedure of } \\
\text { boron reagent }\end{array}$ & Aldol product & Yield (\%) \\
\hline 1 & $\mathrm{Bu}_{2}$ BOTf & $\begin{array}{l}\text { Commercial } \\
\text { product }\end{array}$ & $($ rac $)$-18 & No conversion \\
\hline 2 & $(+)-(\mathrm{Ipc})_{2}$ BOTf & $\begin{array}{l}\text { Paterson's } \\
\text { procedure }\end{array}$ & $(S)-\mathbf{1 8}$ & 44.2 \\
\hline 3 & $\mathrm{Cy}_{2} \mathrm{BOTf}$ & $\begin{array}{l}\text { Abiko's proce- } \\
\text { dure }\end{array}$ & $($ rac $)$-18 & No conversion \\
\hline 4 & $\mathrm{Cy}_{2} \mathrm{BCl}$ & $\begin{array}{l}\text { Reider's proce- } \\
\text { dure }\end{array}$ & $($ rac $)$-18 & $\begin{array}{l}17-25 \text { (conversion } \\
\text { determined by } \\
{ }^{1} \mathrm{H} \\
\text { NMR spetra) }\end{array}$ \\
\hline 5 & $\mathrm{Cy}_{2} \mathrm{BCl}$ & $\begin{array}{l}\text { Commercial } \\
\text { product }\end{array}$ & $($ rac $)$-18 & $\sim 40$ \\
\hline
\end{tabular}

Table 3.1: The Use of Commerical and "Homemade" Boron Reagents in the Aldol reactions of Acetone with Methacrolein
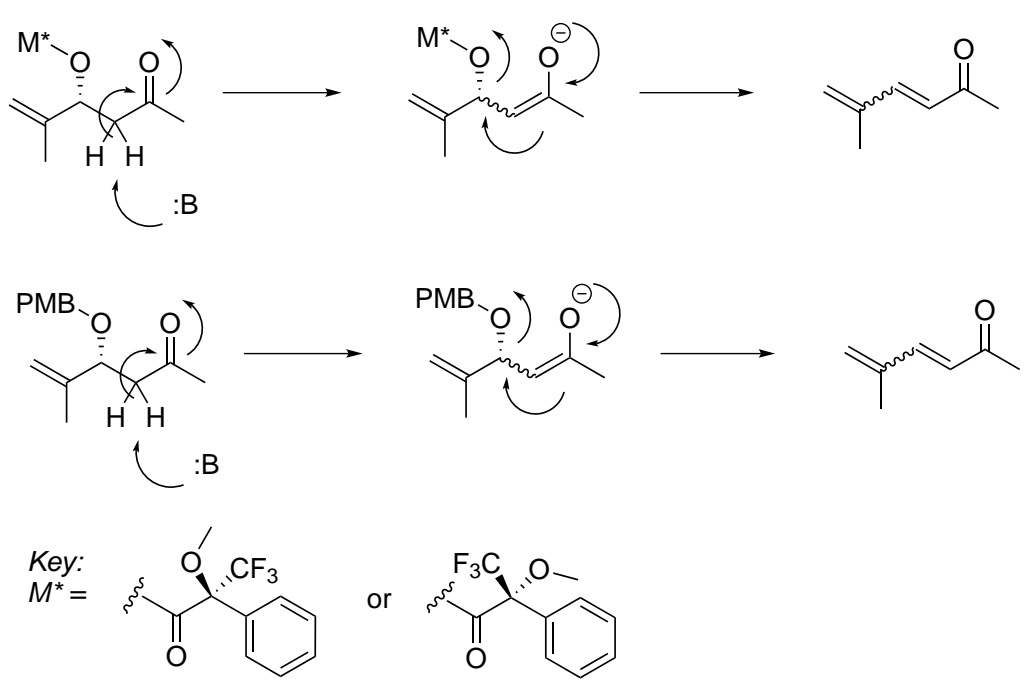

Scheme 3.3: Possible Decomposition Pathways by E1cB Elimination ${ }^{44}$ 


\subsubsection{List's Asymmetric Aldol Reaction and Synthesis of $(R)-18$}

$(R)$-18 has been readily prepared in $38 \%$ yield with a good ee (74\%) (Reaction (2) in Scheme 3.2 ) using $L$-proline as an organocatalyst following the protocol of List's procedure and later used by Hoberg's \& co-workers. ${ }^{4,43}$ The proposed mechanism involves organocatalysis as established by List et al. in Scheme 3.4. ${ }^{43,45}$ The ketone is first transformed to an enamine with $L$-proline, which then reacts with the aldehyde via a Zimmermann-Traxler chair TS. The carbonyl oxygen of the aldehyde is thought to hydrogen-bond to the carboxylic acid and the Re-enatiotopic face of the aldehyde is attacked by the enolate since the $\mathrm{R}$ group can sit at an equatorial position in the resulting TS. This method involves a comparatively simple procedure, can be performed at room temperature (RT), although the expense of the other enantiomer, $D$-proline, potentially limits the utility of this aldol reaction for generating the natural enantiomer $(S)-\mathbf{1 8}$ for peloruside A $[(+)-\mathbf{1}] .{ }^{45}$

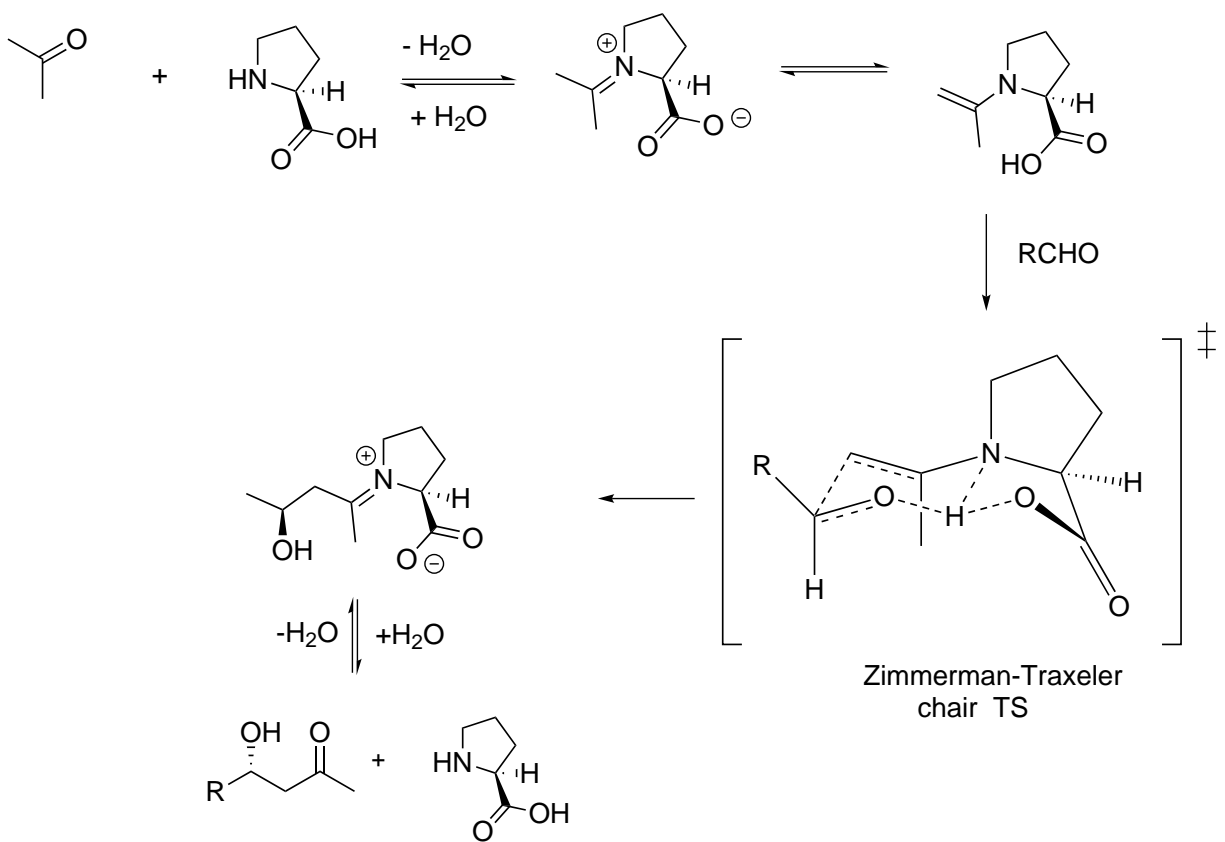

Scheme 3.4: List's Enamine Mechanism of $L$-Proline Catalyzed Asymmetric Aldol Reaction ${ }^{43}$

When this aldol reaction was used in the synthesis of $(R)-\mathbf{1 8}$ for our study, a large amount of DMSO remained in the crude product, complicating the purification and unknown by-products eluted together with $(R)$-18. Fortunately, most of the DMSO was removed by passing the crude product through a pad of silica gel and then the desired $(R)-\mathbf{1 8}$ was purified using fractional distillation $\left(58-60{ }^{\circ} \mathrm{C}, 0-1 \mathrm{mbar}\right)$. The distilled aldol product can be stored under inert gas in a freezer for several months without decomposition. Because of the elimination when esterifying with Mosher ester $(S) \mathbf{- 1 8}$, the ee of $(R)-\mathbf{1 8}$ was not determined. ${ }^{44}$ The $74 \%$ ee determined by Hoberg et al. ${ }^{4}$ (using a $60 \mathrm{~m} \times 0.25 \mathrm{~mm}$ Cyclodex-B column), however, was assumed. 


\subsubsection{Synthesis of Racemic 18}

(rac)-18 was synthesized in a reasonable yield of $65 \%$ after distillation, using LDA as a base (Reaction (3) in Scheme 3.2) according to Stocker's procedure. ${ }^{5}$ The formation of LDA in situ was conducted at $-78{ }^{\circ} \mathrm{C}$ after a significant decomposition happened at $0{ }^{\circ} \mathrm{C}$. The enolization of acetone and the following aldol reaction were also run at $-78^{\circ} \mathrm{C}$. After work-up, the resulting product was distilled with a kugelrohr apparatus under high vacuum (0-1 mbar). When the oven temperature was set at $10{ }^{\circ} \mathrm{C}$ higher than the expected boiling point ${ }^{2}\left(58-60{ }^{\circ} \mathrm{C}, 0-1 \mathrm{mbar}\right)$, by-product(s) were observed in the ${ }^{1} \mathrm{H}$ NMR spectrum, therefore, the temperature of the oven in the kugelrohr distillation needed to be set as close to the boiling point as possible and a second distillation was often required if a large amount was synthesized. Interestingly, although the ${ }^{1} \mathrm{H}$ NMR spectrum of distilled ( $\mathrm{rac}$ )-18 did not show any by-product signal, the stability of distilled (rac)-18 formed by this method was much lower than distilled $(R)-\mathbf{1 8}$ and it was best to use it within two weeks, otherwise a new distillation was required. This suggests that the by-product from this reaction can cause decomposition of ( $r a c)-\mathbf{1 8}$. 


\subsection{Syntheses of Protected $\beta$-Oxygenated Methyl Ketones}

Comparison of Reactions (1) and (2) in Scheme 1.15 (page 26) shows the importance of the two phenyl substituents attached to the silicon in attaining high stereoselectivity. However, it is unclear whether the presence of the eight-membered ring, the second oxygen attached to the silicon (the oxygens, highlighted in red, of $\mathbf{2}$ and $\mathbf{6 a}$ in Reactions (1) and (4) compared to the $t$-butyl group of $\mathbf{5}$ in Reaction (3), Scheme 1.15), or both are essential factors for the high stereoselectivity. ${ }^{2,4}$ In order to solve this puzzle, five methyl ketones with different protecting groups on the $\beta$-alcohol were synthesized (Figure 3.1). Acyclic methyl ketones 19a and $\mathbf{3}$ have the same electronic factors at silicon as $\mathbf{2}$, thus their aldol reactions may reveal the roles of the eight-membered ring and the second oxygen in the asymmetric induction.

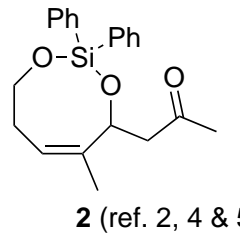

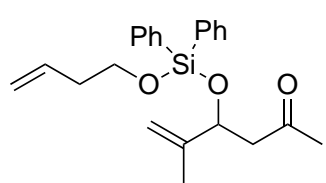

3 (ref. 2, 4 \& 5)

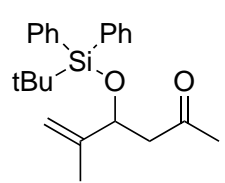

5 (ref. $4 \& 5$ )

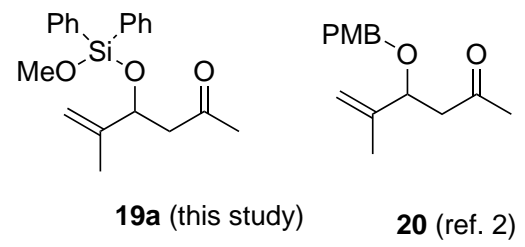

Figure 3.1: Five Methyl Ketones used in the Mechanistic Investigation of 1,5-anti-Aldol Reactions

\subsubsection{Preparation of Various Acyclic Protected $\beta$-Oxygenated Methyl Ke- tones}

Compound $\mathbf{6 b}$, the pre-metathesis substrate for $\mathbf{6 a}$, was synthesized from alcohol $\mathbf{2 1 a}$, dichlorodiphenylsilane $\mathbf{2 2}$ and $(S)-\mathbf{1 8}$ by Turner (Scheme 3.5). ${ }^{2}$ The bulkier alcohol 21a was added first in the reaction in order to minimize the homo-disubstitution of $\mathbf{2 2}$. Therefore, our synthesis of $(R)$ or ( rac)-19a followed this procedure, ${ }^{2,4}$ whereby $(\mathrm{rac}) \mathbf{- 1 8}$ or $(R)-\mathbf{1 8}$ was added to $\mathbf{2 2}$ followed by the methanol. This afforded a moderate yield (30\%) over two steps with many by-products complicating the purification (Reaction (1), Scheme 3.6). As the substitution of the first chloride from dichlorodiphenylsilane $\mathbf{2 2}$ requires refluxing in $\mathrm{CH}_{2} \mathrm{Cl}_{2},{ }^{2,4}$ it is possible that most of the mono-substituted diphenylsilane 23a could be decomposed before the addition of methanol. Therefore, we decided to reverse the addition order by adding the methanol first and the reaction was run overnight at $40{ }^{\circ} \mathrm{C}$ followed by reaction with ( rac)-18 at RT. However, the reaction provided recovered ( $r a c)-\mathbf{1 8}$ and dimethoxydiphenylsilane without any desired product. This indicates that methanol addition to $\mathbf{2 2}$ occurs unselectively and replaces both chlorides prior to addition of $(\mathrm{rac})$-18. $\mathrm{AgNO}_{3}$ has been commonly used to activate silyl halides in protection 
reactions of alcohols, ${ }^{46}$ and so a room temperature reaction was attempted using 4.0 eq $\mathrm{AgNO}_{3}$, 1.1 eq. $\mathbf{2 2}$ and 1.0 eq. ( $r a c)-\mathbf{1 8}$ followed by the addition of 2.0 eq. methanol (Reaction (2), Scheme 3.6). Surprisingly, 19b was obtained without any of the desired product, indicating $\mathrm{AgNO}_{3}$ has a strong activating effect in this substitution. Decreasing the amount of $\mathrm{AgNO}_{3}$ to 2.0 eq. did not provide any improvement and $19 \mathrm{~b}$ was still the major compound isolated, based on the TLC. These results showed that the activating effect from $\mathrm{AgNO}_{3}$ was too strong to allow for mono-substitution but the conditions were mild enough for $(\mathrm{rac}) \mathbf{- 1 8}$ to survive throughout the whole reaction. Nevertheless, (rac)-19a, prepared by Turner's procedure, was used in the following boron-mediated aldol reaction. Compound 19a was not stable in $\mathrm{CDCl}_{3}$ presumably due to the acidic evironment.

In contrast to 19a, homoallylic alcohol-substituted methyl ketone $\mathbf{3}$ was synthesized in a good yield of $65 \%$ over two steps with a modification of Turner's procedure ${ }^{2}$ by adding homoallylic alcohol first followed by $(R)-\mathbf{1 8}$ or (rac)-18 (Reaction (3) in Scheme 3.6). ${ }^{4}$ The steric bulk of homoallylic alcohol is sufficient to enable selective mono-substitution and the resulting mono-substituted ether was stable in refluxing $\mathrm{CH}_{2} \mathrm{Cl}_{2}$.

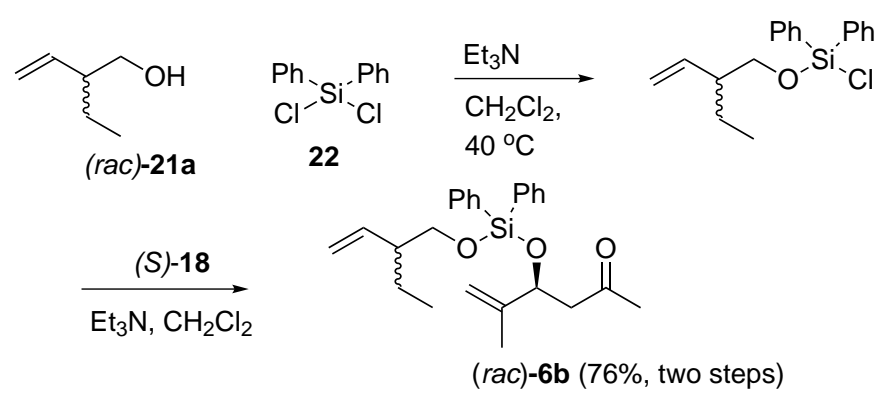

Scheme 3.5: Synthesis of $(r a c)-\mathbf{6 b}$ reported by Turner ${ }^{2}$

In preparation of $(R)$ - and (rac)-5, the introduction of tert-butyldiphenylsilyl group to $(R)$ and (rac)-18 using a standard procedure ${ }^{4}$ was achieved in a moderate yield (54\%) and 2 eq. $t$ - $\mathrm{BuPh}_{2} \mathrm{SiCl}$ was used to since the large steric bulk of this tert-butyldiphenylsilyl chloride caused a difficulty for (rac)-18 to approach (Reaction (1) in Scheme 3.5). The use of 4 eq. $t-\mathrm{BuPh}_{2} \mathrm{SiCl}$ was also tried, however $\mathbf{5}$ and a resulting by-product, presumably the $t-\mathrm{BuPh}_{2} \mathrm{SiOH}$ or the disilyl ether, had the same mobility on silica chromatography. Thus multiple chromatography columns were required to purify the product and the overall yield was lower than that with 2 eq. $t$ - $\mathrm{BuPh}_{2} \mathrm{SiCl}$.

The synthesis of (rac)- and ( $R$ )-20 could not be achieved with 4-methoxybenzyl chloride and (rac)- and $(R)-\mathbf{1 8}$ according to Turner ${ }^{2}$ and Tho, ${ }^{44}$ possibly due to an E1cB elimination caused by the $\mathrm{NaH}$ base that required for this alkylation (Reaction (2), Scheme 3.3 on page 38). However, a good yield (60\%) could be achieved under an acidic condition using 4-methoxybenzyl trichloroacetimidate $\mathbf{2 4}$ and a catalytic amount of TfOH or (rac)-CSA (Reaction (2) in Scheme 


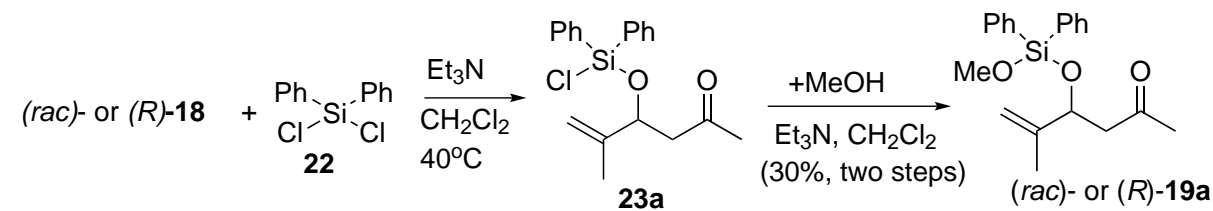<smiles>C=C(C)[C@H](O)CC(=O)CC</smiles>

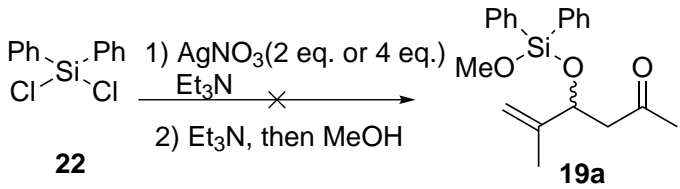

No detection of $19 a$
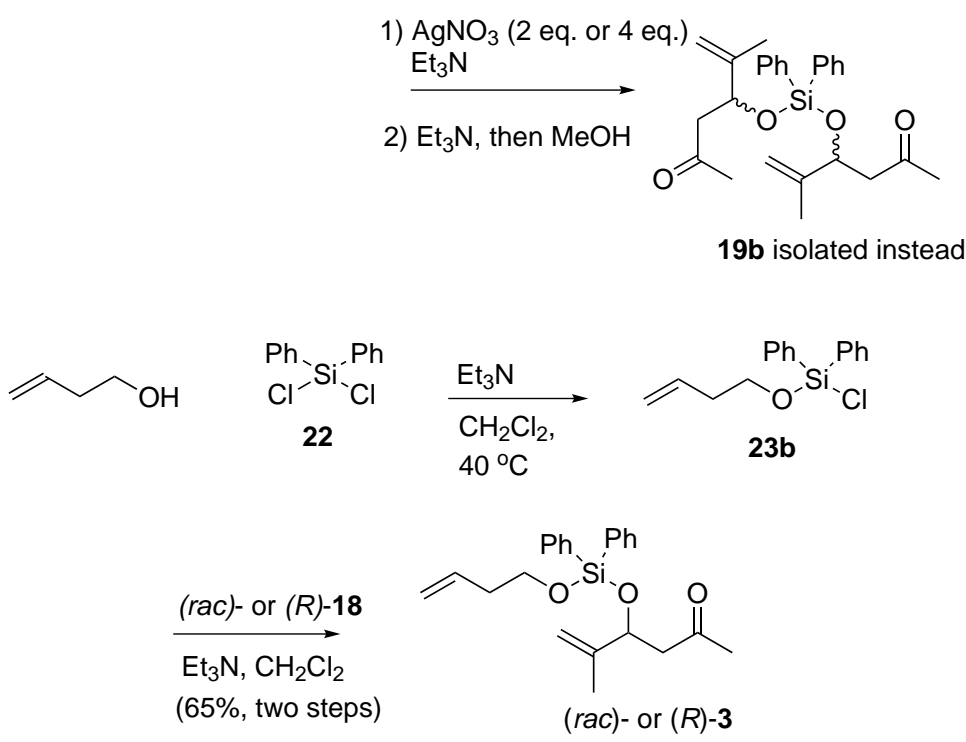

Scheme 3.6: Syntheses of 19a and $\mathbf{3}$

3.7). ${ }^{2}$ The use of ( rac)-CSA ${ }^{46}$ was preferred, because the freshly distilled TfOH was highly hygroscopic and hard to maintain at high purity on storage. The major by-products of this reaction were trichloroacetamide and 4-methoxybenzyl alcohol. Trichloroacetamide was easily removed by dissolving the mixture in hexane and filtering. However, 4-methoxybenzyl alcohol behaved similarly to $\mathbf{2 0}$ in flash chromatography even with different solvent systems. In an attempt to minimize the generation of trichloroacetamide and 4-methoxybenzyl alcohol, the reaction was repeated using 1.0 eq. ( $\mathrm{rac}$ )-18 and 1.0 eq. $\mathbf{2 4}$ but a lower yield (30\%, not pure) was obtained. Therefore, the use of 2.0 eq. $\mathbf{2 4}$ was deemed necessary to afford a good yield. To purify a small quantity of $\mathbf{2 0}(<50 \mathrm{mg})$, preparative TLC was sufficient, otherwise a long flash chromatography column was employed for the purification of more quantity of crude product (>100 mg). Subliming the 4-methoxybenzyl alcohol from the product may be an alternative purification in the future. 

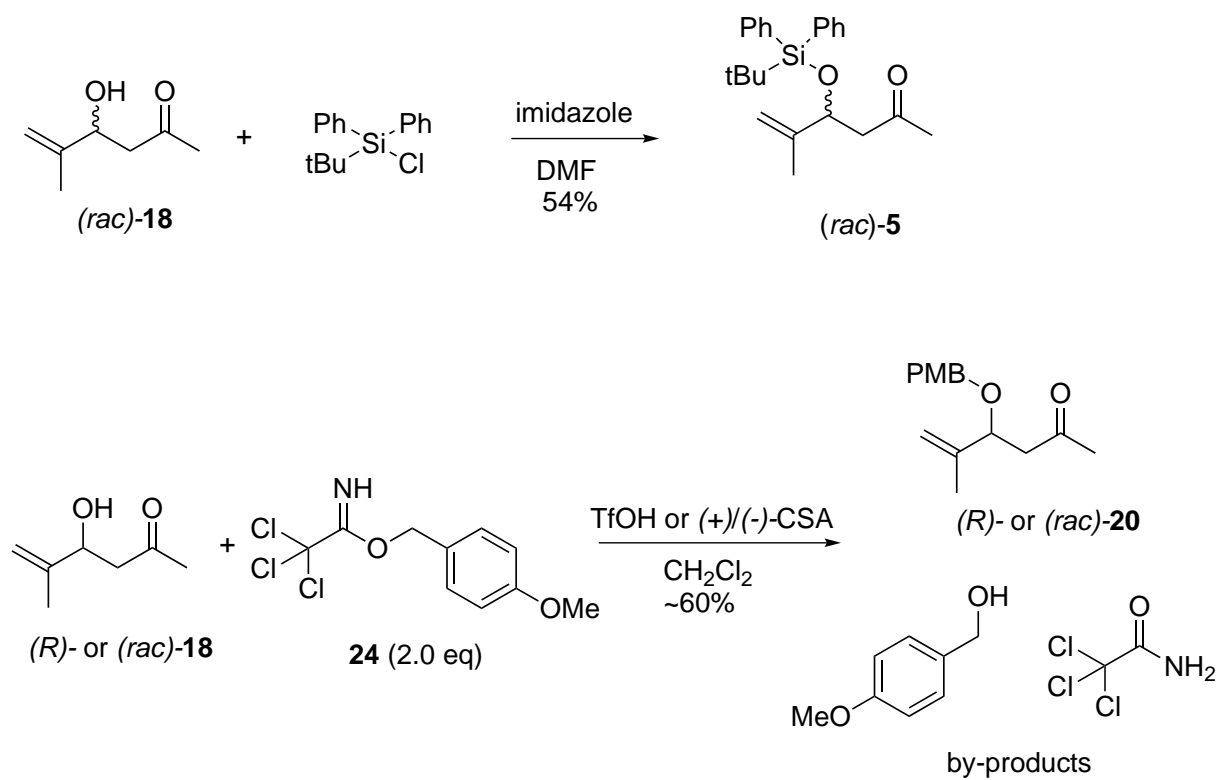

Scheme 3.7: Syntheses of $\mathbf{5}$ and $\mathbf{2 0}^{2,44}$

\subsubsection{Preparation of Cyclic $\beta$-Oxygenated Methyl Ketone 2}

Diphenylsilyl acetal tethered cyclic $\mathbf{2}$ is a simplified variant of ketone $\mathbf{6 a}$ which represents the $\mathrm{C}_{12}$ to $\mathrm{C}_{24}$ fragment of peloruside $\mathrm{A}$ in our synthetic strategy (Scheme 1.15 on page 26). ${ }^{4}$ Among many examples of cyclization reactions in literature, RCM has become increasingly popular in natural product synthesis. However, similar to the other ring closing reactions, enthalpic (ring strain) and entropic (the probability of two alkenes meeting) effects typically lead to a low yield in the formation of a medium-sized (seven- to nine-membered) ring. ${ }^{2}$ In order to assist this type of RCM, different component(s) can be introduced to the system to cause conformational constraint(s) to minimize the unfavorable entropic effect. For example, the presence of a preexisting ring, such as 1,2-disubsituted benzene or Delgado's pyran, ${ }^{47}$ has been used to restrict the torsional angle in order to facilitate the RCM reaction. Alternatively, the dilution factor is also important since the major by-products in medium-sized RCM are cross-metathesis products which sometimes can be prohibited in a highly dilute reaction solution. ${ }^{2,44}$ Silicon-tethered RCM can be a useful method for synthesizing a medium-sized ring. Because the silicon has a bigger size and softer $d$-orbital than oxygen and carbon, the $\mathrm{C}-\mathrm{Si}$ and $\mathrm{O}-\mathrm{Si}$ bonds are easier to distort and fewer steric constraints are encountered during the cyclization. ${ }^{2}$

In Hoberg's retrosynthetic scheme, ${ }^{4}$ acyclic $\mathbf{3}$ was conducted through a ring closing metathesis (RCM) reaction to afford cyclic $\mathbf{2}$. Similar to Hoberg's result, ${ }^{4}$ we only detected the formation of $(Z)$-2 after the metathesis of (rac)-3. Turner proposed a chair TS for the RCM leading to $(S)-\mathbf{6 a}$ based on the nOe correlation of $(S)-\mathbf{6} \mathbf{a} .{ }^{48}$ Presumably, the RCM of $\mathbf{3}$ also adopts a chair conformation during the TS (Reaction (1), Scheme 3.8). ${ }^{2}$ Only a moderate yield (40\%) of (rac)$\mathbf{2}$ was afforded in our study, despite the use of $12 \mathrm{~mol} \%$ catalyst $\mathbf{2 5}$ (Grubbs 2nd generation, Figure 3.2) and a significant amount of $(\mathrm{rac}) \mathbf{- 3}(>40 \%)$ and $(\mathrm{rac}) \mathbf{- 1 8}$ was recovered. The source 
of $(\mathrm{rac}) \mathbf{- 1 8}$ could be due to the deprotection of $(\mathrm{rac}) \mathbf{- 3}$ by activated charcoal used in the work-up step $^{49}$ (discussed later in this section). The yield of this metathesis was lower than what was reported by Hoberg et al. (54\%, $12 \mathrm{~mol} \% \mathbf{2 5}) .{ }^{4}$ This difference could be due to the quality of the catalyst since Turner reported a similar yield $(45 \%, 12 \mathrm{~mol} \% \mathbf{2 5})$ when the same series of bottles of catalysts was used. ${ }^{2}$ However, the major factor that prevented the RCM could be the 1,1-disubstituted alkene since the RCM of $(R)$-26b which has no allylic methyl group gave an excellent yield (81\%) in RCM when only 6 mol\% catalyst was used (Reaction (2), Scheme 3.8). ${ }^{4}$ Turner and Tho likewise discovered that $\mathbf{6 a}$ exhibited a very low activity in RCM. ${ }^{2,44}$ In most of the conditions they tried, only starting materials and cross-metathesis product were obtained. However, by adding the diene $\mathbf{6} \mathbf{b}$ as a $0.01 \mathrm{M}$ solution in $\mathrm{CH}_{2} \mathrm{Cl}_{2}$ to a refluxing solution of $\mathbf{2 5}$ in $\mathrm{CH}_{2} \mathrm{Cl}_{2}$ over $24 \mathrm{~h}$ followed by reflux for a further $24 \mathrm{~h}$, a $53 \%$ yield of $\mathbf{6 a}$ was obtained together with $25 \%$ starting diene and $15 \%$ cross-metathesis product (Reaction (3), Scheme 3.8). ${ }^{2,44}$ The probability of intermolecular metathesis was dramatically decreased by adding a dilute solution of diene slowly to a concentrated catalyst solution and the interaction between diene and catalyst was largely increased so that RCM could afford a better yield. However, repetition of this method in the present work led to only a trace amount of $(\mathrm{rac})-\mathbf{2}$, a poor recovery of the starting diene along with other unknown compounds as evidenced by TLC and ${ }^{1} \mathrm{H}$ NMR spectrum of the crude reaction mixture. This could be due to poor stability of $\mathbf{2}$ and/or $\mathbf{3}$ with heat including possible ring opening metathesis of ( $\mathrm{rac}$ )-2 followed by a cross-metathesis since $\mathbf{2}$ is more sterically accessible for $\mathbf{2 5}$ than $\mathbf{6 a}$ since Tho reported that the cross-metathesis reaction of $\mathbf{6 b}$ was the major side reaction when preparing ketone $\mathbf{6 a}$ using Turner's condition. ${ }^{44}$

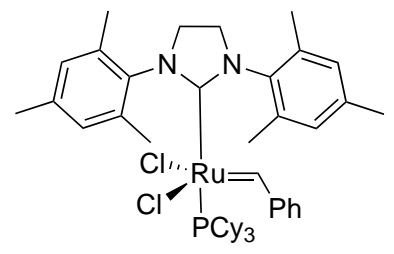

Figure 3.2: Grubbs 2nd Generation Catalyst (25) 

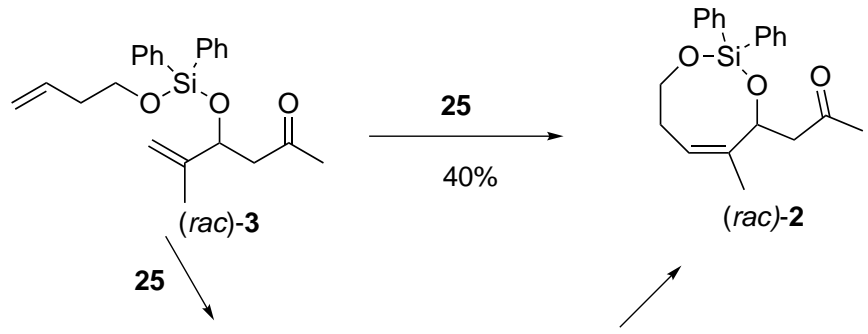

(1) ref. $2,4 \& 5$

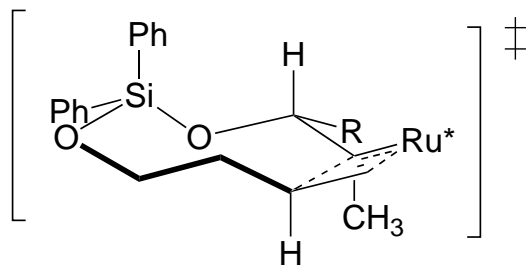

Proposed Chair TS for RCM of 2

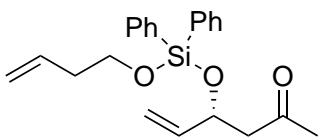

$(R)-26 \mathrm{~b}$<smiles>C=C[C@H](CC)CO[Si](OC)(OC(CC(C)=O)c1ccccc1)C(=C)C</smiles>

$6 b$

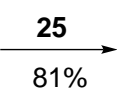

$1 \%$

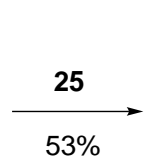

$3 \%$

Key:

$R=\mathrm{CH}_{2} \mathrm{COCH}_{3}$

$R u^{*}=$ Ruthenium complex

Scheme 3.8: Ring Closing Metathesis Reactions ${ }^{4,48}$

It was essential to separate the ruthenium by-product from cyclic 2, otherwise the subsequent aldol reaction would not proceed as reported by Stocker. ${ }^{5}$ Although several methods are reported for this, ${ }^{50-52}$ work here initially followed the precedence of Hoberg ${ }^{4}$ and Turner $^{2}$ in using method of Cho and $\mathrm{Kim}^{49}$ who reported that ruthenium can be removed by passing the reaction mixture through a pad of silica gel followed by treating the eluent with activated charcoal (50 eq. by weight of the reaction mixture) for 24 h. ${ }^{49}$ After filtration, the chromatography is reported to give the desired product with a ruthenium concentration as low as $0.4 \mu \mathrm{g} / 5 \mathrm{mg}$ product. Interestingly, we obtained very different results between the use of granulated and powdered activated charcoal. The use of powdered activated charcoal completely desilylated cyclic ( $r a c)$-2 and the starting diene ( $r a c)$-3 affording the resulting diol and (rac)-18. In contrast, treatment with granulated activated charcoal worked fine for the reaction mixture. The different procedures employed in preparation for commercial activated charcoal could explain these results. For example: acidic conditions are used in the preparation of powdered activated charcoal according to the preparation procedure described on the product information from Sigma-Aldrich. A putative acidic environment on the surface of the charcoal might lead to a 
silyl deprotection. We also discovered that the granulated activated charcoal adsorbed a significant amount of cyclic $\mathbf{2}$ and required a further wash with hot EtOAc, but if too much EtOAc was used, significant ruthenium residue could also be washed out. Because of these difficulties, by testing a number of solvent systems for chromatography, it was discovered that the use of two sequential flash chromatography columns with 12:1 hexane:EtOAc solvent system alone was able to purify $(r a c)-\mathbf{2}$. 


\subsection{Aldol Reactions of Various $\beta$-Oxygenated Ketones}

\subsubsection{Determination of the Relative Stereochemistry of 28a}

Before the mechanism of the stereoselective aldol reaction of $\mathbf{2}$ with aldehydes was investigated, the relative stereochemistry of the aldol product need to be determined which is the primary objective of our study (Chapter 2 on page 34). Stocker has reported a NOESY study of the aldol product when pivalaldehyde was used in this aldol reaction and a 1,5-anti relationship was revealed. ${ }^{5}$ However, this method was not able to determined the stereochemistry of the aldol product in the model study of Peloruside A analogues' $\mathrm{C}_{11}-\mathrm{C}_{12}$ bond formation reported by Turner. ${ }^{2}$ Therefore, we decided to use Kishi's ${ }^{13} \mathrm{C}$ NMR database to determine the stereochemical outcome of the aldol reaction of $\mathbf{2}$.

Cyclic methyl ketone (rac)-2 was enolized with $\mathrm{Cy}_{2} \mathrm{BCl}$ and $\mathrm{Et}_{3} \mathrm{~N}$ followed by the addition of $\mathbf{2 7}$ a to afford aldol product 28a in a moderate yield (49\%, dr $=>99: 1$, Scheme 3.9). Interestingly, the first attempted aldol reaction gave a large amount of by-products and no starting material was recovered; however, in the ${ }^{1} \mathrm{H}$ NMR spectrum of the crude reaction mixture, a number of alkene peaks were detected. Decomposition caused by the deprotection and other complications with the methacrolein were suspected. In the work-up step, hydrogen peroxide solution was used, as is common, to break down the boron complex with the product to form boronic acid. Therefore, free radicals might be generated in this step, reacting with alkene and electron-rich silicon to decompose the compounds. In contrast, alternative work-up conditions with methanol and $\mathrm{pH} 7$ sodium phosphate buffer solution led to no decomposition. In agreement with Hoberg's result, ${ }^{4}$ only one stereoisomer was detected in the ${ }^{1} \mathrm{H}$ NMR spectrum of the crude reaction mixture. In order to determine the stereochemical outcome, it was decided to transform 28a into tetraol 29 (Figure 3.3) so that Kishi's NMR database ${ }^{53}$ could be used to reveal the relative stereochemistry.

In 2000, Kishi's group reported a very interesting database of ${ }^{13} \mathrm{C}$ NMR shifts for 1,3,5-triols systems through their work on palytoxin. ${ }^{53}$ They had experimentally demonstrated that the relative stereochemistry of oxymethine centers had steric and stereoelectronic effects on each other when directly connected or in a 1,3-relationship, whereas the effects were not significant if the connections were two or more $\mathrm{CH}_{2}$ units away. For example, as shown in (1), Figure 3.3 (page 50), the ${ }^{13} \mathrm{C}$ chemical shifts of $\mathrm{C}_{3}$ in syn-diol $\mathbf{3 0 a}$ and syn,syn-triol $\mathbf{3 0 e}$ are very similar in

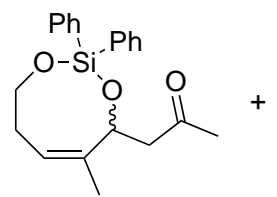

$($ rac)-2

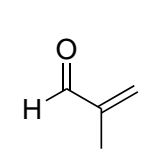

27a

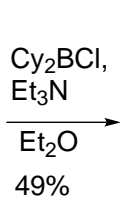

$49 \%$

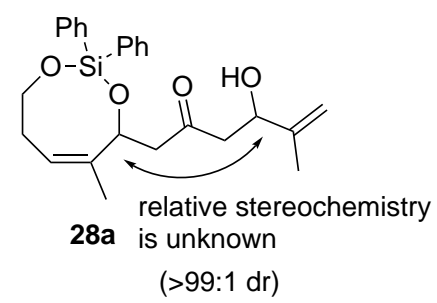

Scheme 3.9: Aldol Reaction of $\mathbf{2}$ with $\mathbf{2 7 a}$ 
both $\mathrm{CD}_{3} \mathrm{OD}$ and $\left(\mathrm{D}_{6}\right)$ DMSO whereas the chemical shift of $\mathrm{C}_{3}$ in $\mathbf{3 0 b}$ which bears an anti-diol was at ca. -2 ppm upfield in both $\mathrm{CD}_{3} \mathrm{OD}$ and $\left(\mathrm{D}_{6}\right) \mathrm{DMSO}$ compared to that of 30a. They also stated that the NMR spectral data for a complex molecule follows the rules obtained from a set of more simpler molecules. For example, as shown in (2), Figure 3.3, both alcohols attached to $\mathrm{C}_{25}$ and $\mathrm{C}_{35}$ in desertomycin $\mathrm{A}$ have the similar relative configuration to that in anti,anti-triol 30h. The corrsponding ${ }^{13} \mathrm{C}$ chemical shifts of $\mathrm{C}_{25}\left(63.7 \mathrm{ppm}\right.$ in $\left.\left(\mathrm{D}_{6}\right) \mathrm{DMSO}\right)$ and $\mathrm{C}_{35}(63.8 \mathrm{ppm}$ $\left(\mathrm{D}_{6}\right) \mathrm{DMSO}$ ) in desertomycin A match the ${ }^{13} \mathrm{C}$ chemical shift of $\mathrm{C}_{5}\left(63.9 \mathrm{ppm}\right.$ in $\left.\left(\mathrm{D}_{6}\right) \mathrm{DMSO}\right)$ of anti, anti-triol $\mathbf{3 0 h}$ nicely. The anti,syn oxymethine center at $\mathrm{C}_{27}\left(66.7 \mathrm{ppm}\right.$ in $\left.\left(\mathrm{D}_{6}\right) \mathrm{DMSO}\right)$ also has a close ${ }^{13} \mathrm{C}$ chemical shift to that of $\mathrm{C}_{5}$ of $\mathbf{3 0 g}\left(66.0 \mathrm{ppm}\right.$ in $\left.\left(\mathrm{D}_{6}\right) \mathrm{DMSO}\right)$. The use of these two empirical rules in predicting the relative stereochemistry of 1,3,5-triols showed promise in a general sense, although it did not work well for certain cases, such as the transannular interactions in small and medium rings interfering the prediction using Kishi ${ }^{13} \mathrm{C}$ NMR database which is based on acyclic 1,3,5-triols and macrocycles. ${ }^{53}$

As Figure 3.3 displays, the chemical shifts of the central oxymethine carbons (indicated by arrows) varied according to the relative stereochemistry of the surrounding stereo-centers. All of the data described in Figure 3.3 were obtained with either $(\mathrm{D})_{6} \mathrm{DMSO}$ or $\mathrm{CD}_{3} \mathrm{OD}$. Some interesting trends can be immediately picked out:

- By comparing 30a and $\mathbf{3 0 b}$, it can be seen that the ${ }^{13} \mathrm{C}$ chemical shifts of the central oxymethines ((1), Figure 3.3) in syn-diols are ca. +2 ppm downfield relative to the corresponding anti-diols in both $\left(\mathrm{D}_{6}\right) \mathrm{DMSO}$ and $\mathrm{CD}_{3} \mathrm{OD}$. Furthermore, the relative difference in shifts remains constant within the acyclic system although the absolute chemical shifts are different (30c and 30d).

- The chemical shift difference between syn- and anti-diols is cumulative. By comparing the central carbons of $\mathbf{3 0 e}, \mathbf{3 0 f}, \mathbf{3 0 g}$ and $\mathbf{3 0 h}((2)$, Figure 3.3), one can pick out an array of increments in chemical shift of the center carbon. Relative to the chemical shift of anti, anti-triol 30h, syn,syn-triol 30e was ca. +4 ppm downfield ( $\operatorname{3yn} /$ syn, $2+2=4 \mathrm{ppm})$ and syn,anti-triol $\mathbf{3 0 f}$ and anti,syn-triol $\mathbf{3 0 g}$ were discovered to be ca. $+2 \mathrm{ppm}$ downfield (syn/anti and anti/syn, $2+0$ and $0+2=2 \mathrm{ppm})$.

- According to Kishi's empirical rules, the chemical shifts of the central carbons of the 1,3,5triols are not affected by the functional groups that are connected by two $\mathrm{CH}_{2}$ units away, in other words, the chemical shift of the central oxymethine carbon in the 1,3,5-triols is relatively constant and predictable.

The structure of tetraol $\mathbf{2 9}$ (Figure 3.3) has similarities with compounds in Kishi's method. Thus we expected that tetraol $\mathbf{2 9}$ which could be generated via a two-step modification of aldol product 28a would match the pattern of Kishi's database and allow determination of the relative stereochemistry of $\mathbf{2 8 a}$. 


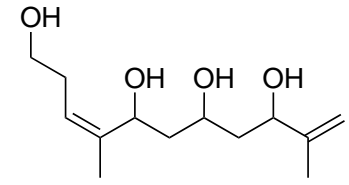

29 (this study)<smiles>CCCC(O)CC(O)/C=C/CO</smiles>

$71.7\left(\mathrm{CD}_{3} \mathrm{OD}\right)$

$69.0\left(\left(D_{6}\right)\right.$ DMSO

$30 \mathrm{c}$<smiles>C=CCC(O)CC(O)CCO</smiles>

$69.1\left(\mathrm{CD}_{3} \mathrm{OD}\right)$ $66.5\left(\left(\mathrm{D}_{6}\right) \mathrm{DMSO}\right)$ $30 a$<smiles>CCCC(O)CC(C)(C)CCO</smiles>

$66.9\left(\mathrm{CD}_{3} \mathrm{OD}\right)$

$64.4\left(\left(\mathrm{D}_{6}\right) \mathrm{DMSO}\right)$

30b<smiles>CCCC(O)C[C@@H](O)/C=C/CO</smiles><smiles>CCCC(O)CC(O)(CC(O)CCO)CC(O)(C(=O)O)C(C)(C)C</smiles>

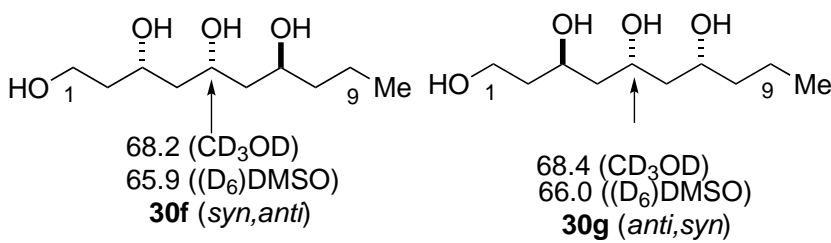<smiles>CCCC(O)C[C@H](O)CC(O)CCO</smiles>

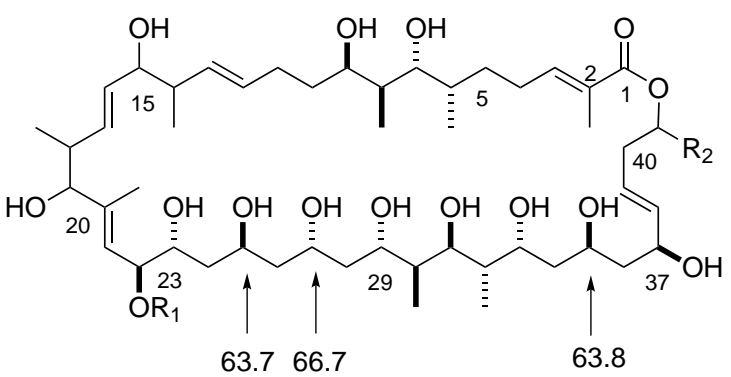

Desertomycin A ((D6) DMSO)

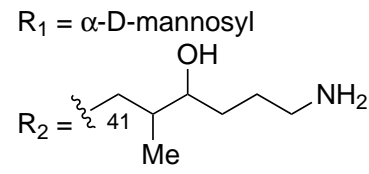

Key:

The ${ }^{13} \mathrm{C}$ chemical shifts are in ppm.

The relative stereochemistry of 29 is not shown but will be deduced in the following discussion.

Figure 3.3: Kishi's ${ }^{13} \mathrm{C}$ NMR Database of $1,3,5$-Triols ${ }^{53}$

Preparation of 29 was achieved by transformation of 28 a to diol 31 by a stereoselective reduction followed by a desilylation. Evans-Chapman-Carreira reduction ${ }^{54}$ of a ketone with a $\beta$-alcohol using tetramethylammonium triacetoxyborohydride in a solution of acetonitrile and acetic acid has often been used in natural product synthesis due to its convenient reaction procedure and high level of 1,3-anti stereoselectivity. The alcohol of 28a was expected to direct the reduction of the ketone to form the 1,3-anti-diol in Evans-Chapman-Carreira reduction. 
According to the TSs proposed by Evans et al. (Scheme 3.10), if the reduction is going through a 1,3-syn TS, the R group on the six-membered chair TS sits in an axial position, interacting unfavorably with acetoxy substituent of the boron reagent. In contrast, the 1,3-anti-TS has the $\mathrm{R}$ group in an equatorial position to prevent the unfavorable 1,3-diaxial interaction although the axial electronegative carbonyl oxygen may have moderate repulsion to the acetoxy substituent. Because the $\mathrm{R}$ group of $\mathbf{2 8} \mathbf{a}$ would be the silyl acetal tethered eight-membered ring which is much bulkier than the carbonyl oxygen, the major product of this reduction is expected to be 1,3-anti-31. Evans-Chapman-Carreira reduction of 28a with 8 eq. $\mathrm{Me}_{4} \mathrm{NHB}(\mathrm{OAc})_{3}$ in a solution of acetonitrile:acetic acid 4:1 was attempted first. The resulting ${ }^{1} \mathrm{H}$ NMR spectrum of the crude reaction product showed a low stereoselectivity $(\mathrm{dr}=3: 1)$ which is unusual since the typical diastereoselectivity for this reduction is $>9: 1$ anti:syn as reported by Evans et al. ${ }^{54}$ It was also reported that an increase in the quantity of acetic acid can enhance the anti-selectivity moderately and the effect appears to level off at $50 \%$ acetic acid in the reaction solvent. ${ }^{54}$ Therefore, the reaction was repeated with more acetic acid (acetonitrile:acetic acid 1:1) but a similar stereochemical outcome $(\mathrm{dr}=3: 1)$ was obtained. These results imply an alternative TS was
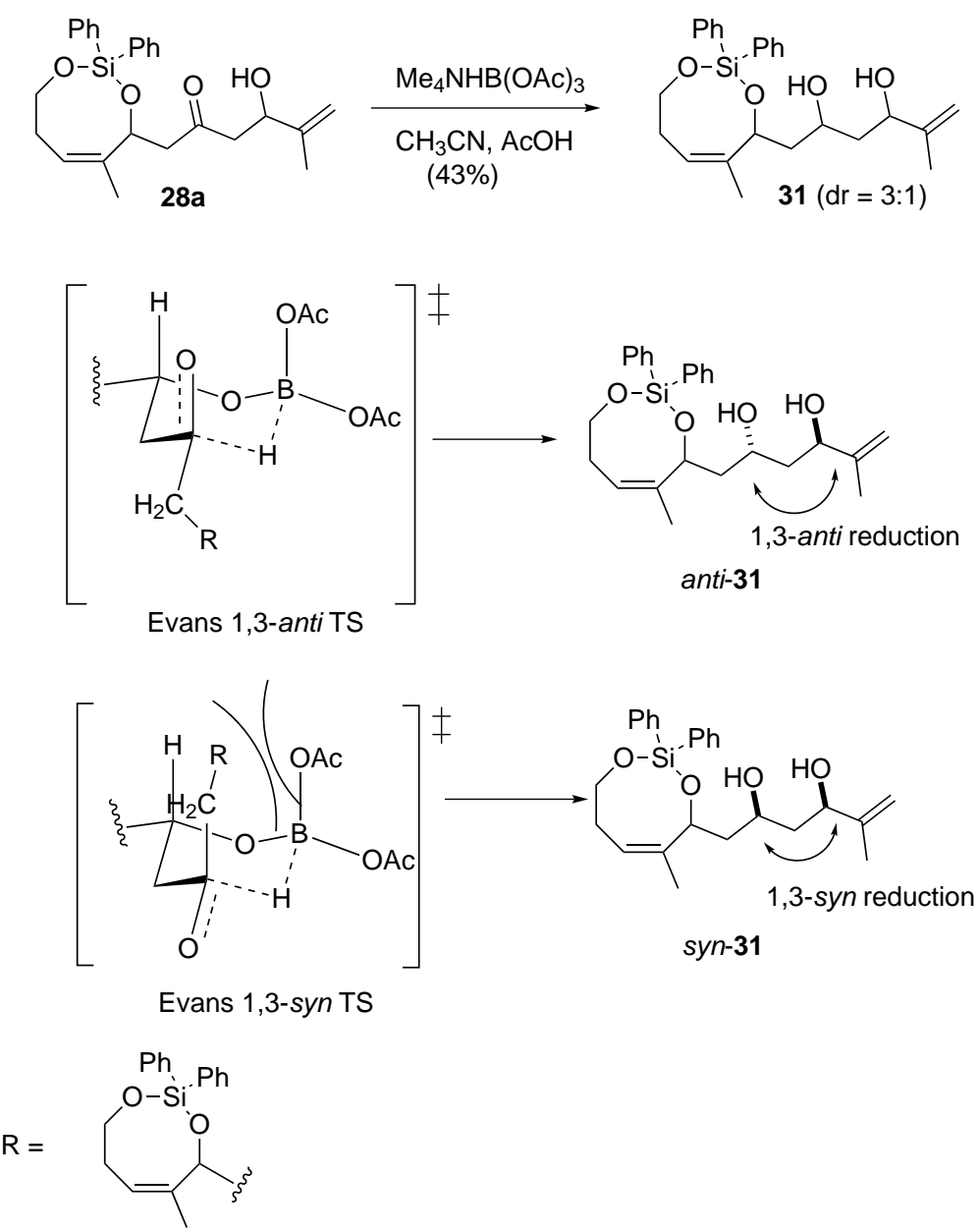

Scheme 3.10: Evans-Chapman-Carreira Reduction of $\mathbf{2 8 \mathbf { a } ^ { 6 }}$

competing with Evans 1,3-anti TS in this reaction. For example, a modified Goodman-type 


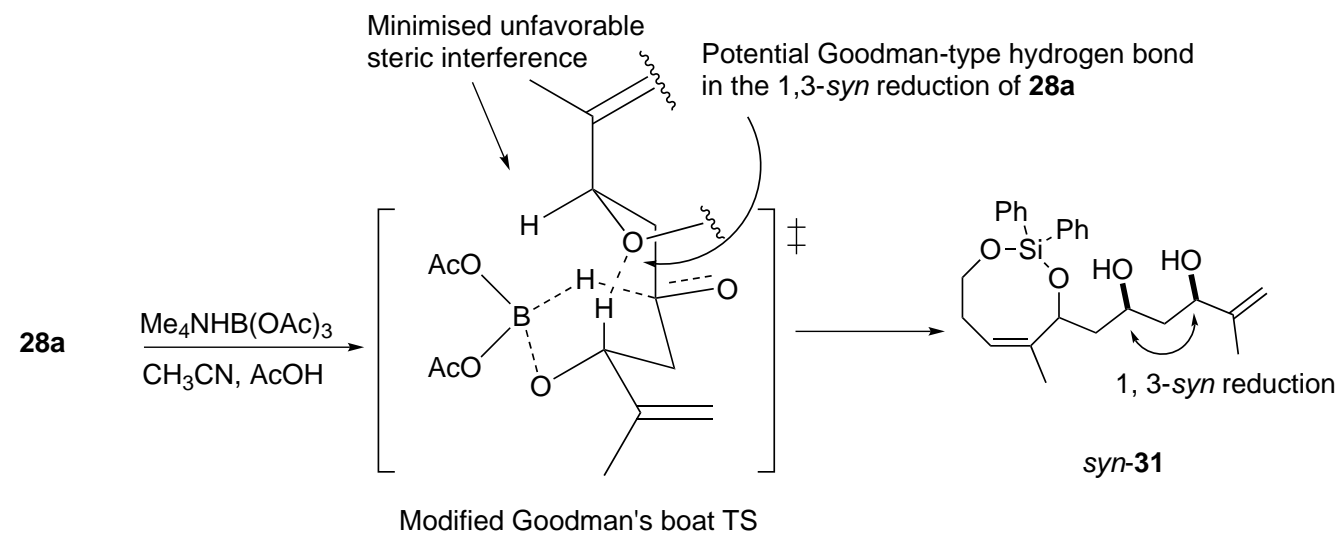

Scheme 3.11: Modified Goodman's Boat TS for Evans-Chapman-Carreira Reduction of 28a ${ }^{6}$

boat could be favored in the TS, producing 1,3-syn 31 (Scheme 3.11). This contains a nonclassical hydrogen bond stabilizing the boat conformer, and the 1,5-anti relationship of $\mathbf{2 8} \mathbf{a}$ ensures the small $\beta$-hydrogen points towards the acetoxy group of the boron reagent. Therefore, this modified Goodman's boat TS is both sterically and electronically favorable and plausibly competes with the Evans 1,3-anti TS. Thus, the relative stereochemistry of the major reduction product was unknown at this stage but it was tentatively revealed after the desilylation of $\mathbf{3 1}$ as described below.

Diol 31 was treated with TBAF to remove the diphenylsilyl protecting group (Scheme 3.12). The reaction mixture was purified with a HP20 column and then silica chromatography, but the purification was not complete since the resulting ${ }^{1} \mathrm{H}$ NMR spectrum showed the signals relating to tetrabutylammonium residues and significant by-product signals were observed at 66-72 ppm in the ${ }^{13} \mathrm{C}$ NMR spectrum. Therefore, the product was further purified with preparative TLC (5:1 $\left.\mathrm{CH}_{2} \mathrm{Cl}_{2}: \mathrm{MeOH}\right)$ to afford the major stereoisomer $(\mathbf{2 9})$ in a pure form and a $34 \%$ yield (the minor isomer was not isolated after the purification).

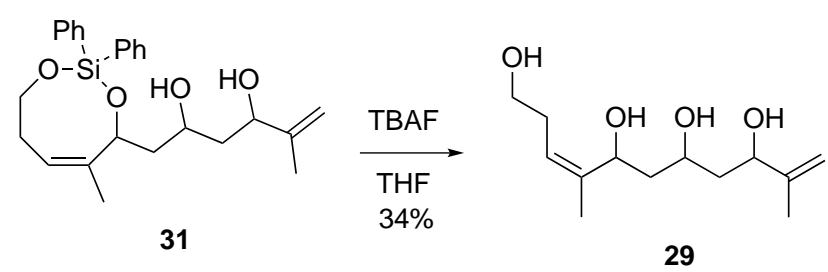

Scheme 3.12: Deprotection of $\mathbf{3 1}$

The resulting ${ }^{13} \mathrm{C}$ NMR spectral data of 29 was obtained in $\mathrm{CD}_{3} \mathrm{OD}$ and three peaks at $73.0,68.4,67.5 \mathrm{ppm}$ corresponding to the three oxymethine carbons were observed (Figure 3.4). According to the HSQC spectrum of $\mathbf{2 9}$, the oxymethine carbons at 73.0 and $68.4 \mathrm{ppm}$ show correlations with the allylic oxymethine protons at 4.23 and $4.80 \mathrm{ppm}$, respectively, and in the HMBC spectrum, they also show correlations with the allylic methyl protons at 1.70 and 1.69 ppm $\left(\mathrm{H}_{12}\right.$ and $\mathrm{H}_{13}$ in Figure 3.4), indicating that these two carbons are the allylic oxymethine 
carbons and the carbon signal at $67.5 \mathrm{ppm}$ corresponds to $\mathrm{C}_{5}$ in 29. Furthermore, an HMBC correlation between $\mathrm{H}_{9}(5.30 \mathrm{ppm})$ and the carbon at $68.4 \mathrm{ppm}$ was found, indicating this carbon is $\mathrm{C}_{7}$ and the other allylic oxymethine carbon (73.0 ppm) is $\mathrm{C}_{3}$ in $\mathbf{2 9}$ (Figure 3.4). The chemical environment of $\mathrm{C}_{3}$ and $\mathrm{C}_{7}$ are similar to each other but the difference in their chemical shifts is ca. $4.7 \mathrm{ppm}$, thus implying the hydroxyls have different stereochemical relationships relative to the central hydroxyl at $\mathrm{C}_{5}$. Furthermore, the chemical shift of $\mathrm{C}_{5}(67.5 \mathrm{ppm})$ in $\mathbf{2 9}$ is closer to the chemical shifts of $\mathrm{C}_{5}$ in syn,anti- $(68.2 \mathrm{ppm})$ and anti,syn- $(68.4 \mathrm{ppm})$ triols $\mathbf{3 0 f}$ and $\mathbf{3 0 g}$ compared to those in $\mathbf{3 0 e}$ (syn,syn, $70.4 \mathrm{ppm}$ ) and $\mathbf{3 0 h}$ (anti,anti, $66.3 \mathrm{ppm}$ )(Figure 3.3). Therefore, the syn,syn- and anti,anti-relative configurations are not likely for 29. Finally, compared to the chemical shifts of allylic positions $\mathrm{C}_{4}$ in $\mathbf{3 0 c}\left(71.7 \mathrm{ppm}\right.$ in $\left.\mathrm{CD}_{3} \mathrm{OD}\right)$ and $\mathbf{3 0 d}$ (69.6 ppm in $\mathrm{CD}_{3} \mathrm{OD}$ ) (Figure 3.3), $\mathrm{C}_{3}-\mathrm{OH}\left(\mathrm{C}_{3}: 73.0 \mathrm{ppm}\right.$ ) of $\mathbf{2 9}$ may be in a syn relationship to $\mathrm{C}_{5}-\mathrm{OH}$, while $\mathrm{C}_{7}-\mathrm{OH}\left(\mathrm{C}_{7}: 68.4 \mathrm{ppm}\right)$ is in an anti relationship to $\mathrm{C}_{5}-\mathrm{OH}$. However, the fact that the Kishi database reference compounds, 30c and 30d, have an $(E)$-alkene, whereas, the alkenes in $\mathbf{2 9}$ are (Z)- and terminal, means the assignment of 3,5-syn,5,7-anti-29 is tentative. It is better to prepare the other diastereomers of $\mathbf{2 9}$ to construct the ${ }^{13} \mathrm{C}$ NMR database so that the ${ }^{13} \mathrm{C}$ chemical shifts of $\mathbf{2 9}$ can be more accurately predicted. However, there was insufficient time to obtain the 1,5-syn aldol product during the time available for this study.

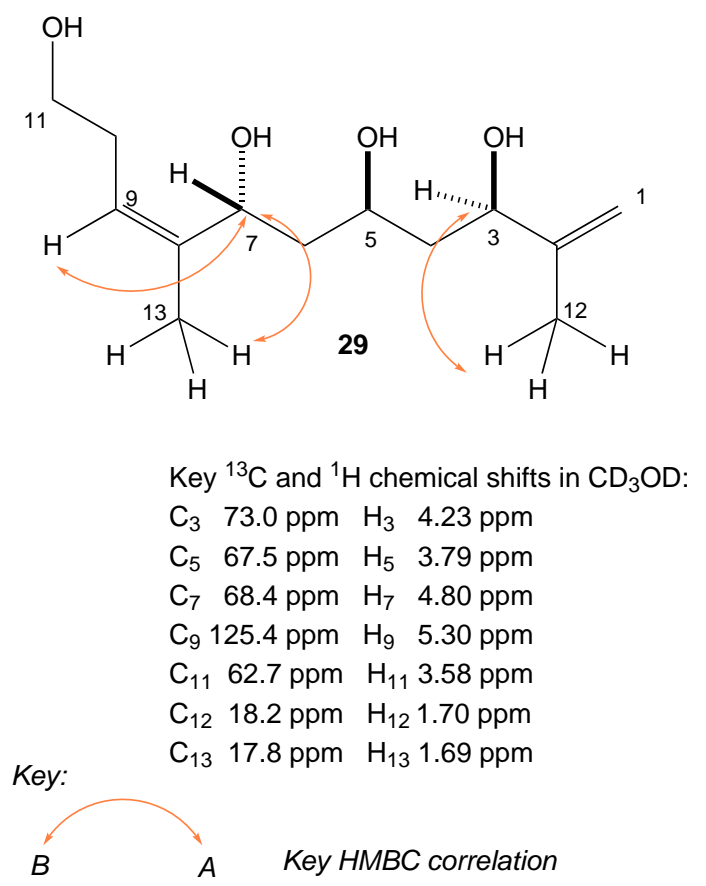

Figure 3.4: Key ${ }^{13} \mathrm{C}$ and ${ }^{1} \mathrm{H}$ NMR Spectral Data and Key HMBC Correlations of 29

The implied anti-relationship between $\mathrm{C}_{3}-\mathrm{OH}$ and $\mathrm{C}_{7}-\mathrm{OH}$ in $\mathbf{2 9}$ suggested that anti-28a has been obtained in the earlier aldol reaction (Scheme 3.13). Interestingly, based on the postulated syn relationship between $\mathrm{C}_{3}-\mathrm{OH}$ and $\mathrm{C}_{5}-\mathrm{OH}$ in 29, 1,3-syn reduction might happen in the reduction of $\mathbf{2 8} \mathbf{a}$, implying the proposed modified Goodman's boat TS might be dominant over the Evans 1,3-anti TS (Schemes 3.10 and 3.11), leading to 1,3-syn 31 as the major product. 


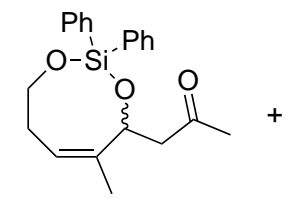

$($ rac) $-\mathbf{2}$

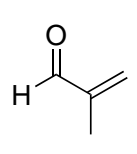

27a

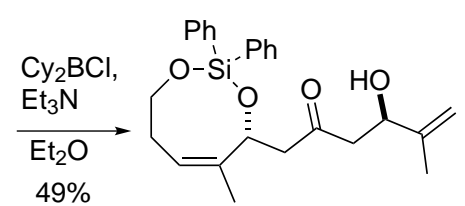

28a (>99:1 anti:syn)

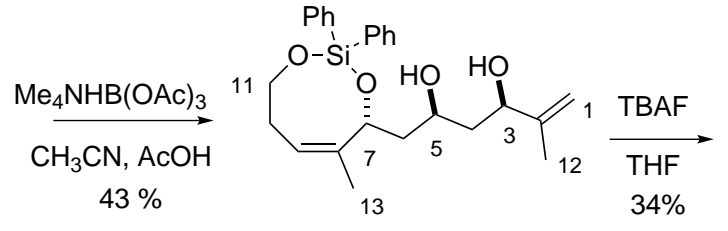

31 (3:1 3,5-syn:3,5-anti)

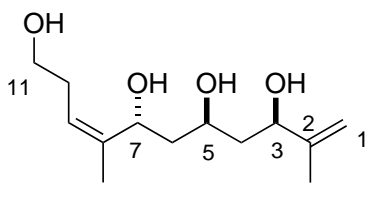

$29($ syn, anti)

Scheme 3.13: Tentative Deduction of the Relative Stereochemistry of 28a and $\mathbf{3 1}$

\subsubsection{Aldol Reactions of Protected $\beta$-Oxygenated Methyl Ketones}

With $\mathbf{3}, \mathbf{5}, \mathbf{1 9} \mathbf{a}$ and $\mathbf{2 0}$ in hand (Figure 3.1 on page 41 ), the next goal was to investigate whether a high 1,5-anti-induction by a non-tethered $\beta$-silyloxy alkyl methyl ketone could be achieved in the aldol reaction. The relative stereochemistry of the aldol products of $\mathbf{3}, \mathbf{5}$ and $\mathbf{1 9 a}$ can be determined as before, by comparison with Kishi's database. These again require a two-step modification of the aldol products which includes an Evans-Chapman-Carreira reduction. ${ }^{54}$ However, the stereochemical outcome of this reduction is not easy to predict as the Goodman's boat-like TS could form with these $\beta$-diphenylsilyloxy alkyl methyl ketones. Therefore, an alternative method was used in the determination of the relative stereochemistry. $\beta$-4-Methoxybenzyloxy alkyl methyl ketones were found to promote a strong 1,5-anti-induction in the aldol reaction as reported by Paterson and Evans, ${ }^{37-39}$ thus their deprotected aldol products, such as from 32a and $\mathbf{3 2} \mathbf{b}$ to $\mathbf{3 3 a}$ and $\mathbf{3 3} \mathbf{b}$, respectively, in Scheme 3.14, could be used as NMR references of anti-diols for determining the relative stereochemistry of the desilylated aldol products of $\mathbf{3}, \mathbf{5}$ and 19a.

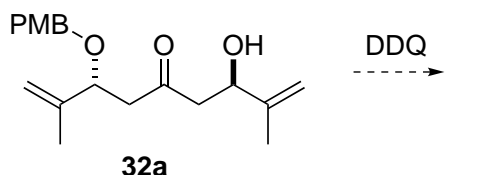

$32 a$

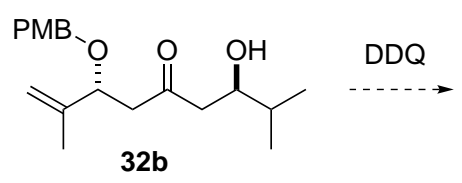<smiles>C=C(C)C(O)CC(=O)CC(O)C(=C)C</smiles>

33a<smiles>C=C(C)C(O)CC(=O)CC(O)C(C)C</smiles>

Scheme 3.14: NMR References of Anti-Diols

Methyl ketone ( $r a c)-\mathbf{2 0}$ was coupled to isobutyraldehyde $\mathbf{2 7 b}$ by a boron-mediated aldol reaction (Scheme 3.15). The aldol product $\mathbf{3 2 b}$ required three flash chromatographies to be 
separated from the boron residues, thus an overall yield of only $24 \%$ was obtained. Crude 32b was stable in $\mathrm{CDCl}_{3}$ and the corresponding crude ${ }^{1} \mathrm{H}$ NMR spectrum showed only one diastereomer, which matched with the literature. ${ }^{2,35,37,38}$

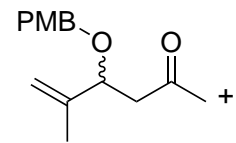

(rac)-20

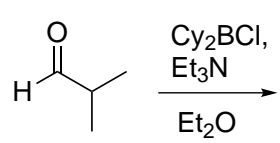

27b

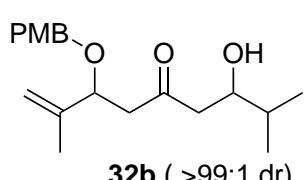

32b ( >99:1 dr)

Scheme 3.15: Aldol Reaction of $\mathbf{2 0}$ with $\mathbf{2 7 b}$

Since we attempted to develop a 1,3-anti diol reference for subsequent NMR comparisons, the relative stereochemistry of $\mathbf{3 2} \mathbf{a}$ and $\mathbf{3 2} \mathbf{b}$ need to be determined first. A two-step chemical modification of $\mathbf{3 2} \mathbf{b}$ to form $\mathbf{3 4}$ was attempted which included 1,3-anti reduction and formation of benzylidene acetal (Scheme 3.16). The formation of a six-membered benzyldiene acetal ring has been commonly used in the determination of the relative stereochemistry of 1,3-diol substrates. ${ }^{46}$ Thus, the relative stereochemistry between $\mathrm{C}_{3}$ and $\mathrm{C}_{5}$ chiral centers of $\mathbf{3 4}$ would be revealed. Furthermore, the asymmetric reduction would ensure an anti-relationship between $\mathrm{C}_{5}$ and $\mathrm{C}_{7}$ chiral centers, thus the overall relative stereochemistry of $\mathbf{3 4}$ and $\mathbf{3 2} \mathbf{b}$ could be revealed.

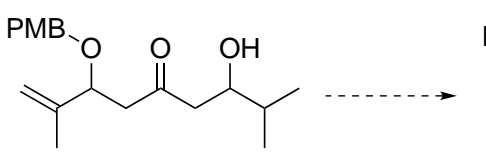

$32 b$<smiles>C=C(C)C(CC(O)CC(O)C(C)C)O[Mg]</smiles>

36

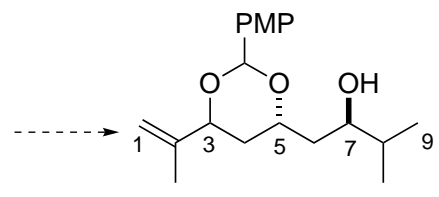

34

Scheme 3.16: A Proposed Two-Step Chemical Modification to Determine the Relative Stereochemistry of $\mathbf{3 2 b}$

4-Methoxybenzylidene acetal-protected 1,3-diols syn- and anti-35 are expected to adopt chair conformers $\mathbf{A}$ and $\mathbf{B}$ or $\mathbf{C}$, respectively (Scheme 3.17). A is more stable than the other possible conformers of syn-35 due to all of the non-hydrogen substituents being in equatorial positions. The nOe correlation between $\mathrm{H}_{1}$ and $\mathrm{H}_{3}$ in this conformer indicates a syn-relationship of the diol. Anti-35 can adopt either chair $\mathbf{B}$ or $\mathbf{C}$, depending on the relative size of $\mathrm{R}_{1}$ and $\mathrm{R}_{2}$. No matter which one is adopted, no nOe correlation between $\mathrm{H}_{1}$ and $\mathrm{H}_{3}$ is expected and a nOe correlation between $\mathrm{H}_{1}$ and $\mathrm{R}_{2}$ or $\mathrm{H}_{3}$ and $\mathrm{R}_{1}$ reveals an anti relationship of the diol. 


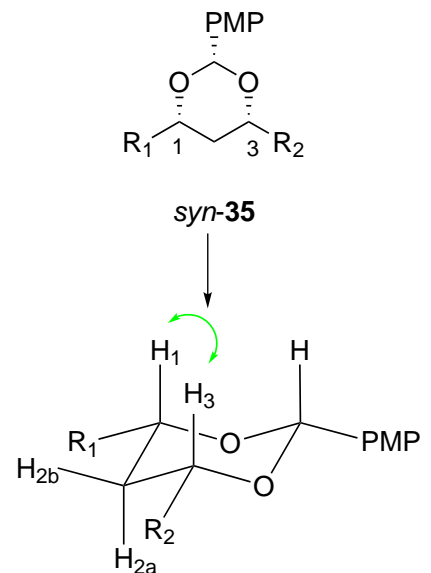

Chair conformer $\mathbf{A}$<smiles>[R16]PC1OC([R6])CC([R8])O1</smiles>

anti-35

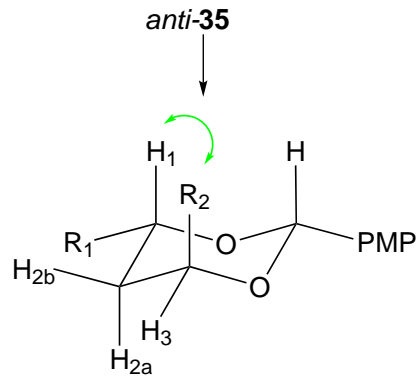

Chair conformer B<smiles>[R]C1CC([R7])OC([R16])O1</smiles>

anti-35

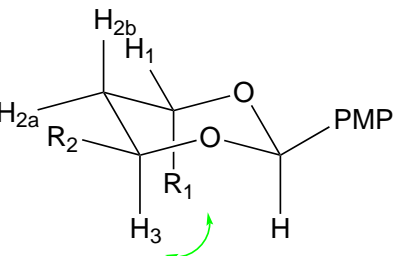

Chair conformer $\mathbf{C}$

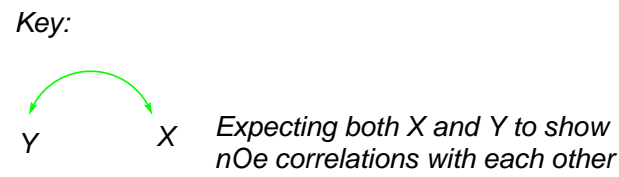

Scheme 3.17: Relative Stereochemistry Determination of 1,3-Diols Using 4-Methoxybenzylidene Acetals $^{46}$

Evans-Chapman-Carreira reduction was used to reduce $\mathbf{3 2 b}$ with the anti-direction from the newly formed alcohol and the resulting product was prepared in a quantitative yield (Reaction (1), Scheme 3.18). Unlike the reduction of 28a, the crude ${ }^{1} \mathrm{H}$ NMR spectrum of the reaction mixture of $\mathbf{3 2} \mathbf{b}$ showed only one diastereomer. According to the literature, ${ }^{54}$ the $\beta$ - 4 methoxybenzyloxy group is unlikely to participate in the stereoinduction in this reduction, so it was assumed that the relative stereochemistry of the diol $\mathbf{3 6}$ was anti. However, the following oxidative cyclization of 4-methoxybenzyl protected $\mathbf{3 6}$ to afford 4-methoxybenzylidine $\mathbf{3 4}$ using DDQ was not successful, generating complicated by-products. 4-Anisaldehyde was detected in the ${ }^{1} \mathrm{H}$ NMR of the crude reaction mixture, which may arise by the over-reduction of PMP group with DDQ, deprotection of $\mathbf{3 4}$ in the work-up step (passage through a pad of silica gel), or in the preparation of the NMR sample with $\mathrm{CDCl}_{3}$.

Methacrolein (27a) was then picked as the alternative standard aldehyde. The corresponding deprotected 1,5-anti-aldol product (anti-33a) was expected to have a $\mathrm{C}_{2}$ symmetric axis but no plane of symmetry (Figure 3.5), which would be active in a polarimeter, in stark contrast, meso syn-33a would be plane-symmetrical (Figure 3.5). Methyl ketone $(R)-\mathbf{2 0}$ was coupled with $\mathbf{2 7 a}$ to afford 32a as a single diastereomer, followed by a PMB-deprotection with DDQ to obtain diol 33a. As expected, 33a showed a significant $[\alpha]_{D}$ activity $\left([\alpha]_{D}^{23}+15.4\left(\mathrm{c} 0.54, \mathrm{CHCl}_{3}\right)\right)$ in the polarimeter, indicating $\mathbf{3 3 a}$ is an anti-diol and the aldol product 32a has the anti configuration (Reaction (2) in Scheme 3.18). 


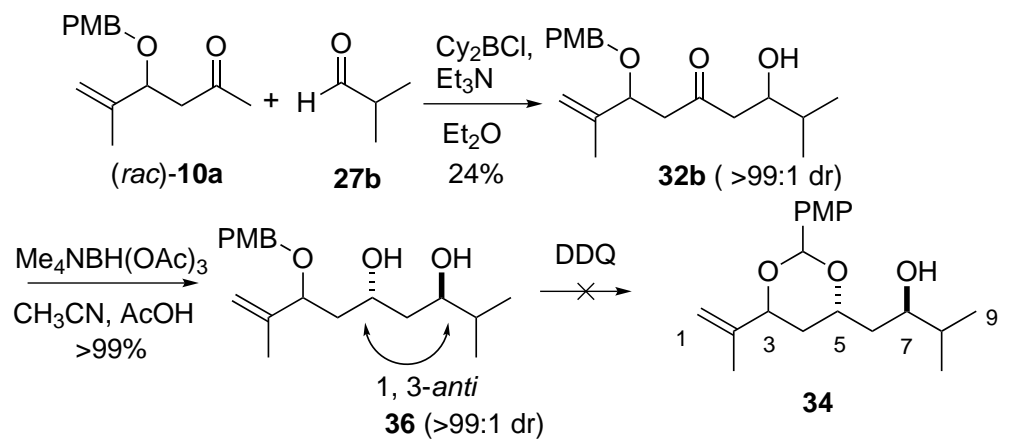<smiles>C=C(C)C(CC(C)=O)O[R16]#N</smiles>

(R)-20<smiles>C=C(C)C=O</smiles>

27a

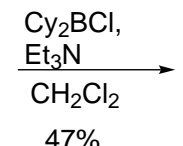

$47 \%$

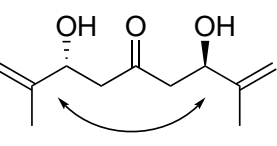

1,5-anti

Polarimeter Active

33a $(>99: 1 \mathrm{dr})$

Scheme 3.18: Aldol Reactions of $\mathbf{2 0}$ with $\mathbf{2 7 a}$ and $\mathbf{2 7} \mathbf{b}$ and their Relative Stereochemistry Determinations

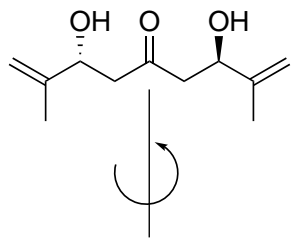

$\mathrm{C}_{2}$ symmetry but no plane of symmetry

anti-33a is chiral

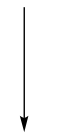

Polarimeter active

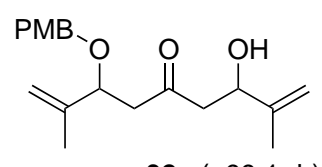

32a $(>99: 1 \mathrm{dr})$
(2)

$$
\begin{gathered}
\underset{\mathrm{DDQ},}{\mathrm{pH} 7 \text { buffer }} \\
\underset{69 \%}{\mathrm{CH}_{2} \mathrm{Cl}_{2}}
\end{gathered}
$$


With the NMR reference of anti-33a in hand, the stereoinduction of methyl ketones $\mathbf{3}, \mathbf{5}$ and 19a were investigated. A boron-mediated aldol reaction of methylsilyl acetal ( $r a c)-19 a$ with methacrolein 27a was carried out using the same conditions as $\mathbf{2}$ (Reaction (1) in Scheme 3.19). Compound 19a has the same electronic properties, having groups with comparable electron demand around silicon, as cyclic $\mathbf{2}$. Thus, the stereochemical outcome of this aldol reaction can reveal the role of the eight-membered ring. Although ( $r a c)-\mathbf{1 9 a}$ was able to couple with $\mathbf{2 7 a}$, the resulting product contained a large amount of silanol 37, which may come from $\mathrm{S}_{N} 2$ attack of hydrogen peroxide, water or methanol on the primary carbon of the methoxy substituent in the work-up step $\left(30 \% \mathrm{H}_{2} \mathrm{O}_{2}\right.$ in water, $\mathrm{pH} 7$ sodium phosphate buffer solution and methanol) . In a second attempt, only the buffer and methanol were added but $\mathbf{3 7}$ was still obtained. Since $\mathrm{Cy}_{2} \mathrm{BCl}$ is a strong Lewis acid and an excess amount was used in this reaction (2.2 eq.), it is possible that the methoxy substituent of the silicon could chelate to the boron so that the primary carbon became more electrophilic, allowing weak nucleophiles, such as water and methanol, to attack this primary carbon (Red arrows in Scheme 3.20). Or alternatively, after the chelation of 19a to boron reagent, the silicon became more electrophilic in which an SN2 substitution could happen in the presence of water in work-up step, leading to by-product $\mathbf{3 7}$ (blue arrows). Similar to the starting ketone ( $\mathrm{rac}$-19a, the aldol product 38a was also unstable in neutralized $\left(\mathrm{NaCO}_{3}\right.$ treated) $\mathrm{CDCl}_{3}$ and an unknown by-product was gradually generated in successive ${ }^{1} \mathrm{H}$ NMR scans. It was also found that the boron residues have similar mobility to $\mathbf{3 8 a}$, thus the purification was not successful with flash chromatography and the yield of this aldol reaction was not determined. Because of the poor quality and stability of the aldol product 38a, homoallyloxy-substituted $\mathbf{3}$ was used instead. This ketone is the substrate for the RCM leading to eight-membered ring $\mathbf{2}$, thus it also contains the same electronic factors at the silicon as $\mathbf{2}$. Compound $\mathbf{3}$ was enolized under the conditions used with $\mathbf{2}$, followed by the addition of $\mathbf{2 7} \mathbf{a}$ to provide the aldol product $\mathbf{3 9} \mathbf{a}$ in a low yield (26\%) and a large amount of recovered starting material (ca. 50\% of 3) (Reaction (2), Scheme 3.19). Interestingly, after a work-up step with $\mathrm{pH} 7$ sodium phosphate buffer solution and methanol, the crude ${ }^{1} \mathrm{H}$ NMR spectrum was relatively clean and no $\mathbf{3 7}$ was found. $\mathbf{3}$ gave a very low stereoinduction in the aldol reaction, namely a 1:1 mixture of anti:syn diastereomers, based on the ${ }^{1} \mathrm{H}$ NMR spectrum of the crude reaction mixture. Both syn- and anti-39a had very similar mobility by the TLC with a variety of solvent systems, thus flash chromatography separated the two diastereomers from the boron residues but not from each other. tert-Butyldiphenylsilyl ether $\mathbf{5}$ was also tried in an aldol reaction with $\mathbf{2 7 a}$ under the same conditions as $\mathbf{3}$ (Reaction (3), Scheme 3.19). Similar to Hoberg et al.'s result, ${ }^{4}$ this aldol reaction provided a very poor stereoselectivity in a ratio of 1.5:1 anti:syn. The stereochemical identity of the major aldol product 40a was determined by comparing the ${ }^{1} \mathrm{H}$ NMR spectral data with anti-33a after a deprotection with TBAF. The major deprotected product has the same ${ }^{1} \mathrm{H}$ NMR spectrum as anti-33a, therefore indicating the 1,5-anti aldol product is the major diastereomer in the aldol reaction. These results indicated that the eight-membered ring of $\mathbf{2}$ is essential for high 1,5-anti stereoinduction and the second oxygen (non- $\beta$-oxygen) of $\mathbf{2}$ is not sufficient in its own right to give a significant stereoinduction. 


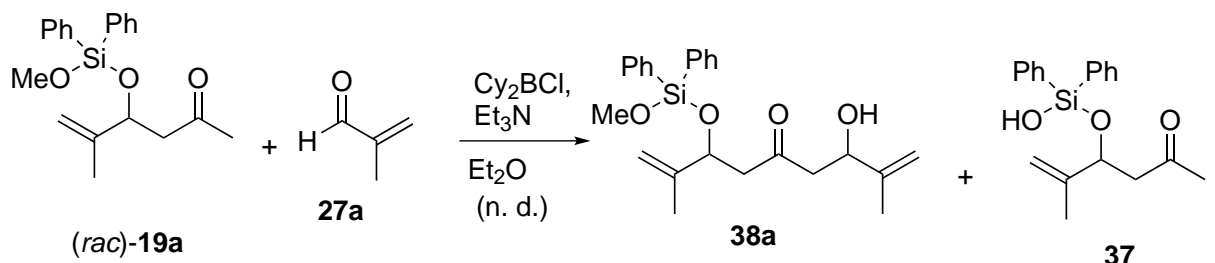<smiles>C=CCCO[Si](OCCC=C)(OC(CC(C)=O)c1ccccc1)C(=C)C</smiles>

(rac)-3<smiles>C=C(C)C=O</smiles>

$27 a$<smiles>C=CCCO[Si](OC(CC(=O)CC(O)C(=C)C)c1ccccc1)(C(=C)C)c1ccccc1</smiles>

39a (1:1 anti:syn)

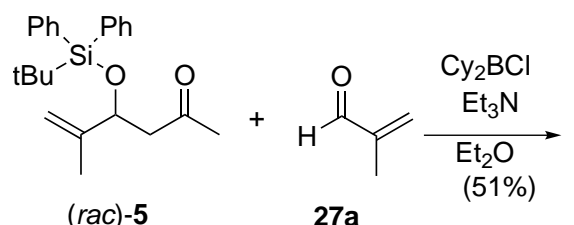

(rac) -5

27a

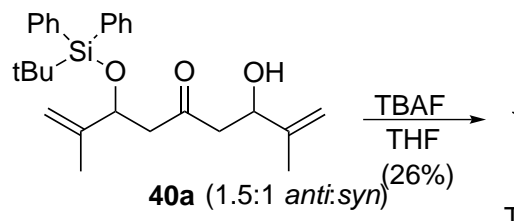<smiles>C=C(C)C(O)CC(=O)CC(O)C(=C)Cl</smiles>

The ${ }^{1} \mathrm{H}$ NMR spectrum of the major product matches that of anti-33a, indicating the major aldol product $40 \mathrm{a}$ has an anti relative stereochemistry.

Key:

n. $d .=$ Yield was not determined

Scheme 3.19: Boron-Mediated Aldol Reactions of 19a, $\mathbf{3}$ and $\mathbf{5}$ with Isobutyraldehyde and Methacrolein

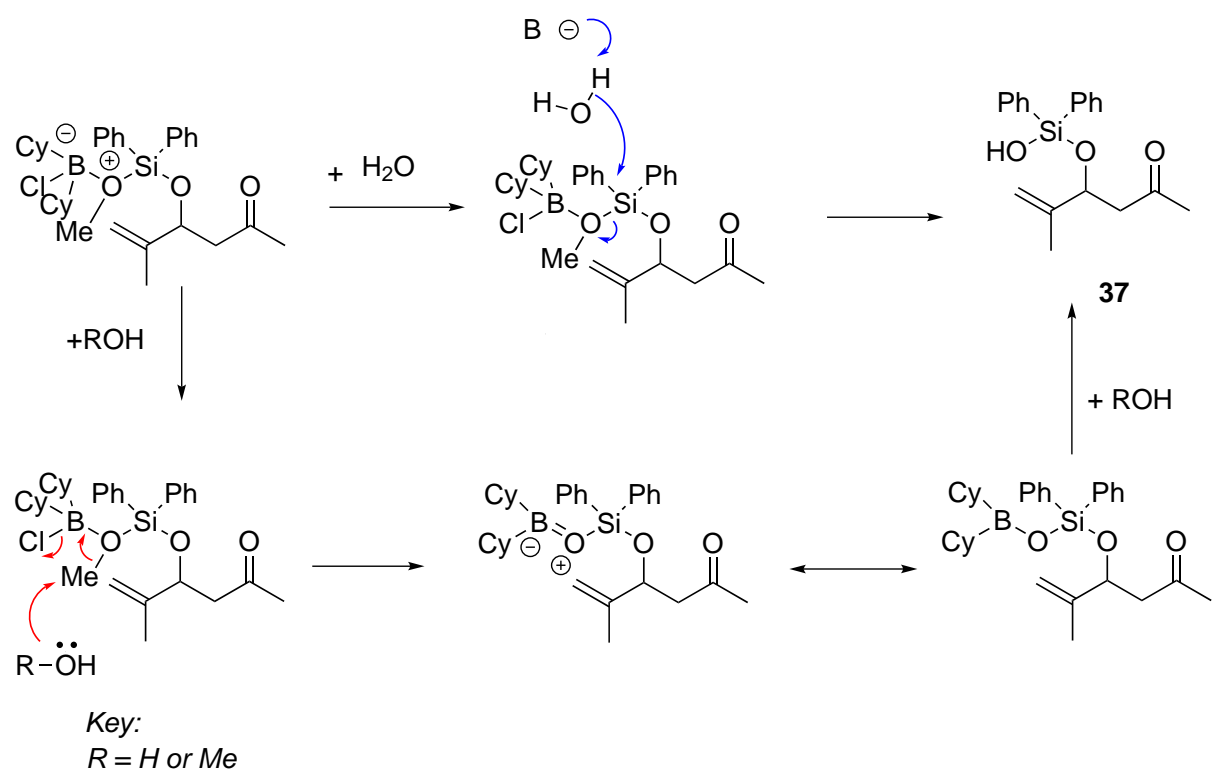

Scheme 3.20: Possible Decomposition Pathway for 19a in the Work-up Step

\subsubsection{Aldol Reactions of 3 Mediated by Lithium and Titanium}

In addition to the structure of the methyl ketone and the type of enolates generated, the correct metal-mediating agent is also essential for the stereoselecivity of the aldol reaction since 
different sized metals may afford different conformations in the TS and the number of chelating sites on the metals can also be different. If the low stereoselectivity of $\mathbf{3}$ was due to a mismatching between the boron reagent and ketone 3, changing to another metal might help to improve the stereoselectivity. Therefore, we decided to explore the use of $\mathrm{LDA}$ and $\mathrm{TiCl}_{4}$ in this aldol reaction. A typical Li-O bond $(1.9 \AA-2.2 \AA)$ is longer than a $\mathrm{B}-\mathrm{O}$ bond $(1.4 \AA)$ in the chelated six-membered ring, therefore, the lithium mediated aldol TS should be less compact. This may make it easier for the methyl ketone $\mathbf{3}$ to adopt a conformation that is beneficial for the stereoinduction, if there is any. However, a similar yield (26\%) was obtained in the aldol reaction and no improvement of the stereoselectivity (1:1 anti:syn) was detected in the crude ${ }^{1} \mathrm{H}$ NMR (Reaction (1), Scheme 3.21).

In contrast to boron reagents, the titanium ion can bond to multiple oxygens in the TS and an octahedral titanium complex is often formed as opposed to the tetrahedral structure of the boron complex. Sometimes multiple titanium atoms can participate in a single TS. ${ }^{55,56}$ These differences can have important consequences for the stereoinduction. For example, according to Ghosh et al. ${ }^{56}$ model $\mathbf{A}$ in Scheme 3.22 leads to a different stereochemical outcome compared to model $\mathbf{B}$ because the $\alpha$-benzyloxy group of the aldehyde also chelates to the titanium in the TS such that a different enantiotopic face of the aldehyde is attacked by the titanium enolate. Therefore, when titanium is mediating the aldol reaction of $\mathbf{3}$, the oxygen of the homoallylic substituent of the silicon of $\mathbf{3}$ may also chelate to the titanium such that the movement of this substituent is greatly restricted and does not interfere with the stereoinduction of $\mathbf{3}$ in the TS (Reaction (2), Scheme 3.21). The aldol reaction of $\mathbf{3}$ with 4 -bromobenzaldehyde $\mathbf{2 7} \mathbf{d}$ provided an excellent yield $(>99 \%)$ but the stereochemical outcome (1.3:1 anti:syn) was still similar to the previous cases in which methacrolein were used as the aldehyde. Two conclusions can be drawn based on these results:

- The poor stereoselectivity outcomes of the boron-mediated aldol reactions of $\mathbf{3}$ should not be attributed to a mismatch between the methyl ketone and the size of the TS caused by the boron reagent $\left(\mathrm{Cy}_{2} \mathrm{BCl}\right)$ in the reaction (Reaction (2), Scheme 3.19) and the eightmembered ring of cyclic $\mathbf{2}$ was again proven to be essential for the excellent stereoinduction.

- It is possible that the bulk of methyl ketone $\mathbf{3}$ may not be accommodated well in the compact lithium-and boron-mediated TS and the reaction did not proceed with a good yield. It is not clear whether the excellent yield of the titanium mediated aldol reaction of $\mathbf{3}$ with 27d (Reaction (2), Scheme 3.21) was due to the large size of titanium minimizing steric interference within the six-membered ring of the TS, and/or the multidentate chelation of 3 to titanium ${ }^{56}$ preventing the flexible homoallylic substituent from interfering the TS or simply the presence of the electrophilic aryl aldehyde. 


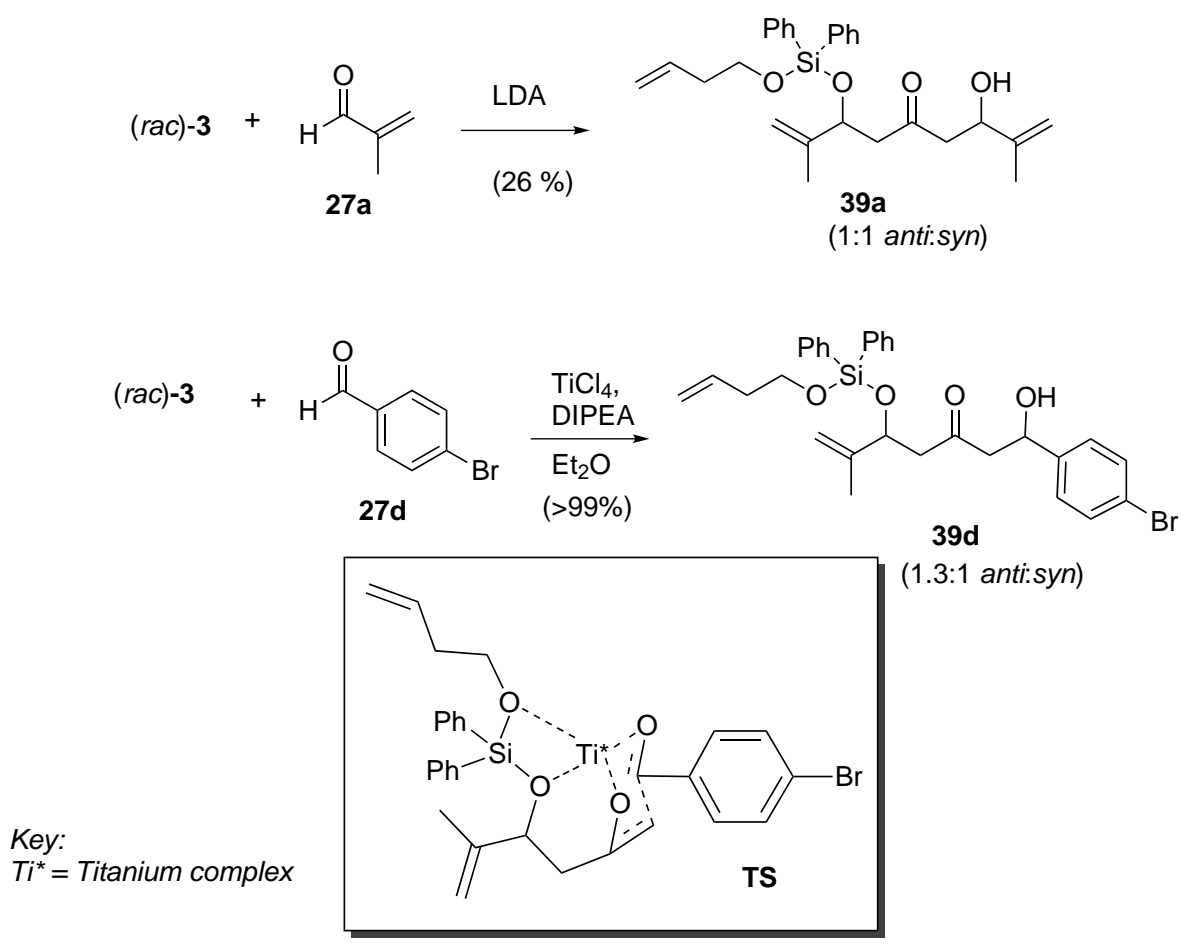

(1)

Scheme 3.21: Aldol Reactions of ( $r a c$ )-3 with Methacrolein 27a and 4-Bromobenzaldehyde 27d using Different Mediating Agents ${ }^{55,56}$

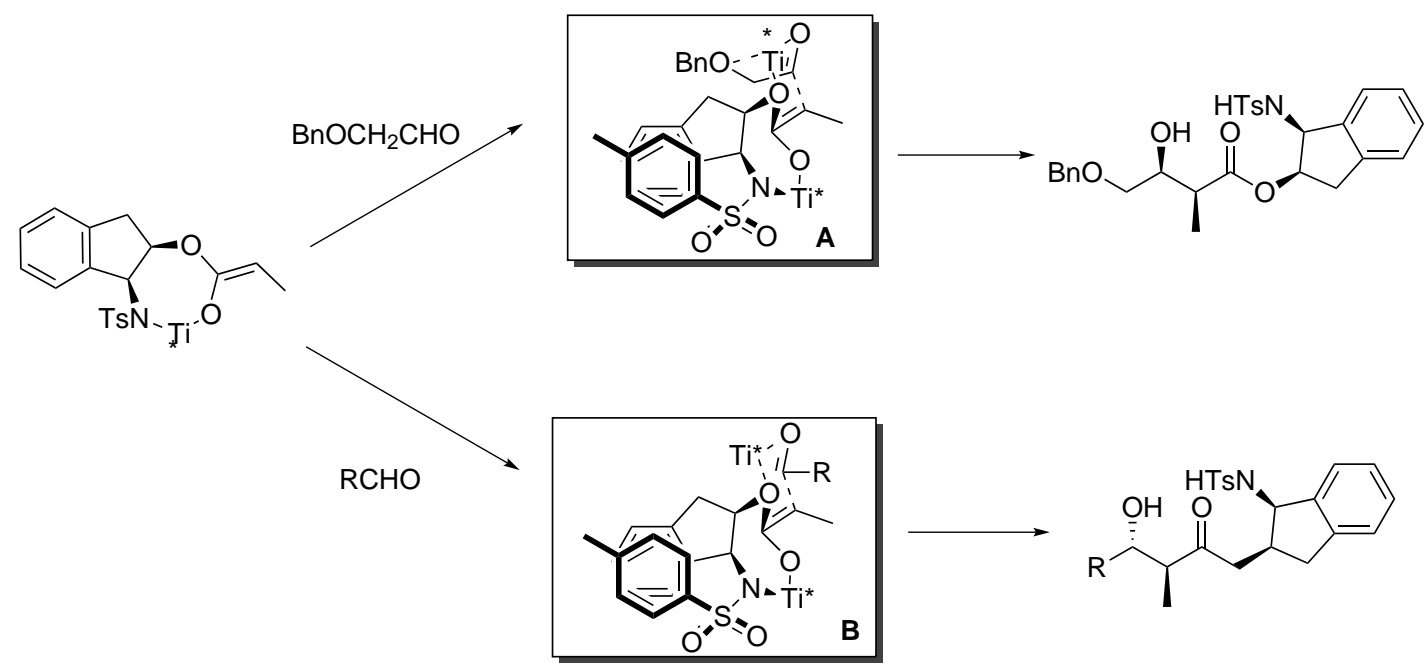

Key:

$T i^{\star}=$ Titanium complex

Scheme 3.22: Titanium-Mediated Aldol Reactions Reported by Ghosh et al. ${ }^{56}$ 


\subsubsection{Questions About Goodman's model}

As described in Section 1.4.2 on page 26, Goodman et al. argue that the unfavorable steric interference between the six-membered ring in the TS and the bulky $\beta$-silyloxy group (TMSOused in their calculation) together with the electronic withdrawing effect on the $\beta$-oxygen from the empty $d$-orbital of the silicon, weaken the Goodman's hydrogen bond and lead to unstable boat TSs. Therefore the chair TS dominates over the boat TS and the similar stabilities of synand anti-chair TSs lead to a low stereoselective aldol reaction. ${ }^{6}$ However, this argument does not match our observation since the diphenylsilyl acetal tethered eight-membered ring of $\mathbf{2}$ is much bulkier than the TMSO group in their calculation, but an excellent 1,5-anti stereoinduction still occurs.

As argued by Turner, ${ }^{2}$ a strengthened Goodman-type hydrogen bond could be formed in the TS derived from 2 because of a reduction in the electron-withdrawing nature of the silicon due to stabilization by the $\pi$-electrons of the phenyl rings. However, if this strengthened hydrogen bond compensated for the steric bulk of the diphenylsilyl acetal group of $\mathbf{2}$, a similar compensation should also happen to 3. However, dramatically different stereochemical outcomes were obtained. Thus, Goodman's model alone is not able to explain the stereoinduction of $\mathbf{2}$, and the conformation of the silyl-acetal tethered eight-membered ring of $\mathbf{2}$ needs to be taken into account in the TS. Therefore confomational studies of $\mathbf{2}$ and $\mathbf{3}$, their boron enolates and aldol products with a variety of NMR experiments were carried out, as described in Section 3.5.

\subsubsection{Summary: Section 3.4}

In summary, methyl ketones $\mathbf{2}, \mathbf{3}, \mathbf{5}, \mathbf{1 9} \mathrm{a}$ and 20 were prepared. Methoxy-substituted 19a and its aldol product are fragile in silica flash chromatography and $\mathrm{CDCl}_{3}$. Although aldol reactions were not always successful, two clear findings were obtained:

1. The highly diastereoselective aldol reaction ( $\mathrm{dr}>99: 1)$ of $\mathbf{2}$ with methacrolein $(\mathbf{2 7 a})$ using $\mathrm{Cy}_{2} \mathrm{BCl}$ and $\mathrm{Et}_{3} \mathrm{~N}$ giving aldol product 28a has been shown to occur, favoring the 1,5-anti stereoselectivity, as previously proposed and studied. ${ }^{2,4,5}$

2. Under the same reaction conditions as $\mathbf{2}$, homoallyloxy-substituted $\mathbf{3}$ gave a very low stereoinduction in the aldol reaction (1:1 anti:syn), indicating the silyl acetal tethered eight-membered ring of $\mathbf{2}$ is essential for the 1,5-anti stereoinduction.

In the process of determining the relative stereochemistry of $\mathbf{2 8 a}$, the ${ }^{13} \mathrm{C}$ NMR spectroscopic data sugested that the reduction of the aldol product 28a with $\mathrm{Me}_{4} \mathrm{NHB}(\mathrm{OAc})_{3}$ gave the unexpected 1,3-syn reduction product. This observation is tentatively explained by a modified Goodman's boat TS.

Although key questions regarding stereoinduction in these aldol reactions have been investigated and in line with Stocker's and Turner's studies, ${ }^{2,4,5}$ but it is unfortunate that the aldol 
reaction of ( $\mathrm{rac}$-19a was not performed successively to add further insight into the influences on these systems. There were two more factors required further investigation. These are the confomations of cyclic $\mathbf{2}$ and acyclic $\mathbf{3}$ in the reaction solution, which will be addressed by NMR studies of $\mathbf{2}$ and $\mathbf{3}$ and their corresponding aldol reaction intermediates and products, as described in Section 3.5. 


\subsection{Conformational Studies Using Various NMR Experiments}

\subsubsection{Conformational Studies of 2 and 3}

To reveal the mechanism of the stereoinduction by $\mathbf{2}$, it would be instructive to determine the conformations of the cyclic and acyclic ketones, $\mathbf{2}$ and $\mathbf{3}$. Although Turner postulated that a chair conformation is adopted in formation of the eight-membered silyl acetal of $\mathbf{6 a}$ with an ethyl substituent, ${ }^{2,48}$ the conformation of $\mathbf{2}$ is still unclear so analysis of $\mathbf{2}$ by nOe was carried out. For the ease of discussion, Table 3.2 shows only the key nOe correlations and ${ }^{13} \mathrm{C}$ and ${ }^{1} \mathrm{H}$ NMR spectral assignments for $\mathbf{2}$. All of the nOe correlations are illustrated in Figure 3.6.

Only the four most common conformers of a silyl acetal-containing eight-membered ring ${ }^{57}$ were considered in this conformational study and consist of two crown-like conformers, the boat conformer and the chair conformer (Figure 3.7). Since $\mathrm{C}_{4}$ of $\mathbf{2}$ is the only chiral center, it was expected that the attached carbon chain $\left(\mathrm{CH}_{2} \mathrm{COCH}_{3}\right)$ would be in the equatorial position. The axial $\mathrm{H}_{4}$ shows a number of nOe correlations and is essential for the conformational determination of the silyl-acetal tethered eight-membered ring of $\mathbf{2}$. The nOe correlations of $\mathrm{H}_{4}$ with the phenyl protons and the allylic methyl protons $\left(\mathrm{H}_{9}\right)$ are important because in Boat conformer of $\mathbf{2}$, such correlations are not expected. Furthermore, the correlation between one or both of $\mathrm{H}_{8}$ and the phenyl protons is also not expected in Boat conformer but detected in our study. This evidence indicates $\mathbf{2}$ does not adopt a boat conformation in solution. In Crown $\mathbf{A}$ conformer, $\mathrm{H}_{4}$ is far away from $\mathrm{H}_{9}$ and phenyl protons, therefore the nOe correlations of $\mathrm{H}_{4}$ indicate Crown $\mathbf{A}$ conformer is also not a major contribution.

Chair conformer (Figure 3.7) can explain all of the correlations, however, the presence of an axial phenyl group, the unfavorable transannular and eclipsing strains (conformation $\mathbf{C}$, Figure 3.7) mean a moderate distortion of the chair would be expected. If $\mathrm{C}_{7}-\mathrm{C}_{8}$ was rotated by changing from conformation $\mathbf{C}$ to $\mathbf{D}$ (Figure 3.7), the "twist-chair" would experience a ring strain and Crown $\mathbf{B}$ conformer could be formed to release this ring strain (Figure 3.7), which also satisfies all of the nOe data. Although minimizing the unfavorable eclipsing strain, Crown B conformer has a large transannular strain since one of the phenyl groups points inside the ring. The coupling pattern of $\mathrm{H}_{8 b}$ is dt in which the doublet with a coupling constant of 11 $\mathrm{Hz}$ corresponds to the geminal coupling between $\mathrm{H}_{8 a}$ and $\mathrm{H}_{8 b}$, and the vicinal couplings of $\mathrm{H}_{8 b}$ with $\mathrm{H}_{7 a}$ and $\mathrm{H}_{7 b}$ appear as a triplet. This indicates the dihedral angles of $\mathrm{H}_{8 b}-\mathrm{C}_{8}-\mathrm{C}_{7}-\mathrm{H}_{7 a}$ and $\mathrm{H}_{8 b}-\mathrm{C}_{8}-\mathrm{C}_{7}-\mathrm{H}_{7 b}$ are similar. Confirmation of this was obtained through homo-decoupling NMR experiments: by irradiating $\mathrm{H}_{7 a}$ and $\mathrm{H}_{7 b}$ one at a time, the resulting peaks of $\mathrm{H}_{8 b}$ gave very similar decoupled patterns. Both the conformations of $\mathrm{C}_{7}-\mathrm{C}_{8}$ bonds in Chair and Crown B conformers fit these observations (Conformations $\mathbf{C}$ and $\mathbf{D}$ ). However, we are still unable to resolve which conformer is adopted by the eight-membered ring based on these data. A molecular mechanics conformational search was therefore undertaken. The nOe correlations were used to generate flat-bottomed constraints based on an expected less than $4 \AA$ between separation of the nOe correlating protons. A Monte Carlo Multiple Minimium-based search provided a Crown 


\begin{tabular}{|c|c|c|c|}
\hline Position & $\delta^{13} \mathrm{C}(\mathrm{ppm})$ & $\delta^{1} \mathrm{H}(\mathrm{ppm})$ & $\begin{array}{l}\text { Key nOe Correla- } \\
\text { tions }\end{array}$ \\
\hline 1 & 30.9 & $2.24(\mathrm{~s}, 3 \mathrm{H})$ & $\mathrm{H}_{4}$ \\
\hline 2 & 207.6 & - & \\
\hline $3 a$ & 49.6 & $2.61(\mathrm{dd}, \mathrm{J}=15.1,3.7 \mathrm{~Hz}, 1 \mathrm{H})$ & \\
\hline $3 b$ & 49.6 & $2.93(\mathrm{dd}, \mathrm{J}=14.9,9.5 \mathrm{~Hz}, 1 \mathrm{H})$ & \\
\hline 4 & 71.7 & $4.89(\mathrm{dd}, \mathrm{J}=9.5,3.4 \mathrm{~Hz}, 1 \mathrm{H})$ & $\begin{array}{l}\mathrm{H}_{1}, \mathrm{H}_{3 a}, \mathrm{H}_{3 b}, \mathrm{H}_{7 b} \text { and } \\
\text { phenyl } \mathrm{H}\end{array}$ \\
\hline 5 & 140.8 & - & \\
\hline 6 & 125.1 & $5.43(\mathrm{t}, \mathrm{J}=8.8 \mathrm{~Hz}, 1 \mathrm{H})$ & \\
\hline $7 \mathrm{a}$ & 31.2 & $2.93(\mathrm{~m}, 1 \mathrm{H})$ & $\mathrm{H}_{6}$ \\
\hline $7 \mathrm{~b}$ & 31.2 & $2.26(\mathrm{~m}, 1 \mathrm{H})$ & $\mathrm{H}_{4}$ and $\mathrm{H}_{6}$ \\
\hline $8 \mathrm{a}$ & 64.4 & $4.02($ app. td, $\mathrm{J}=10.1,3.1 \mathrm{~Hz}, 1 \mathrm{H})$ & $\begin{array}{l}\mathrm{H}_{8 a} \text { and/or } \mathrm{H}_{8 b} \text { corre- } \\
\text { lates with phenyl } \mathrm{H}\end{array}$ \\
\hline $8 \mathrm{~b}$ & 64.4 & $4.07($ app. $\mathrm{dt}, \mathrm{J}=11.0,4.6 \mathrm{~Hz}, 1 \mathrm{H})$ & \\
\hline 9 & 21.5 & $1.60(\mathrm{~s}, 3 \mathrm{H})$ & \\
\hline
\end{tabular}

Table 3.2: Key ${ }^{13} \mathrm{C}$ and ${ }^{1} \mathrm{H}$ Chemical Shifts and nOe Correlations of $\mathbf{2}$
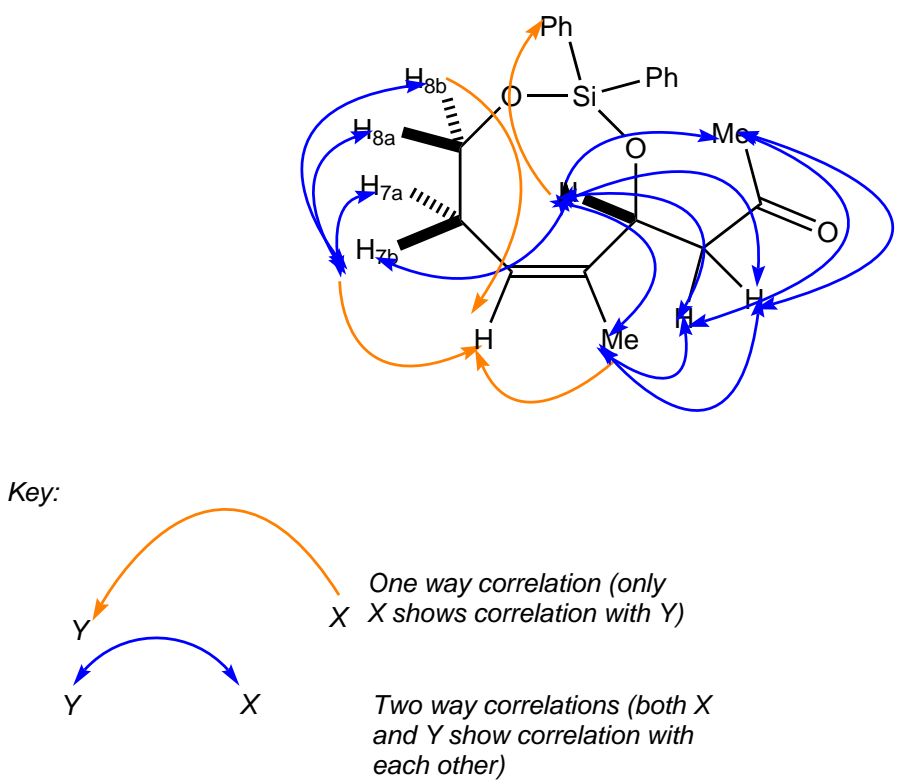

Figure 3.6: Full nOe Correlations of $\mathbf{2}$ 


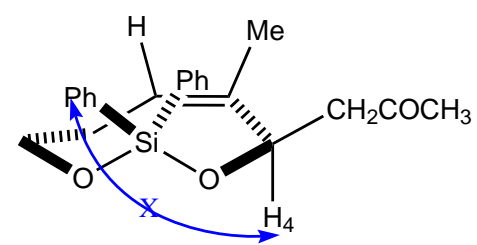

Crown A conformer
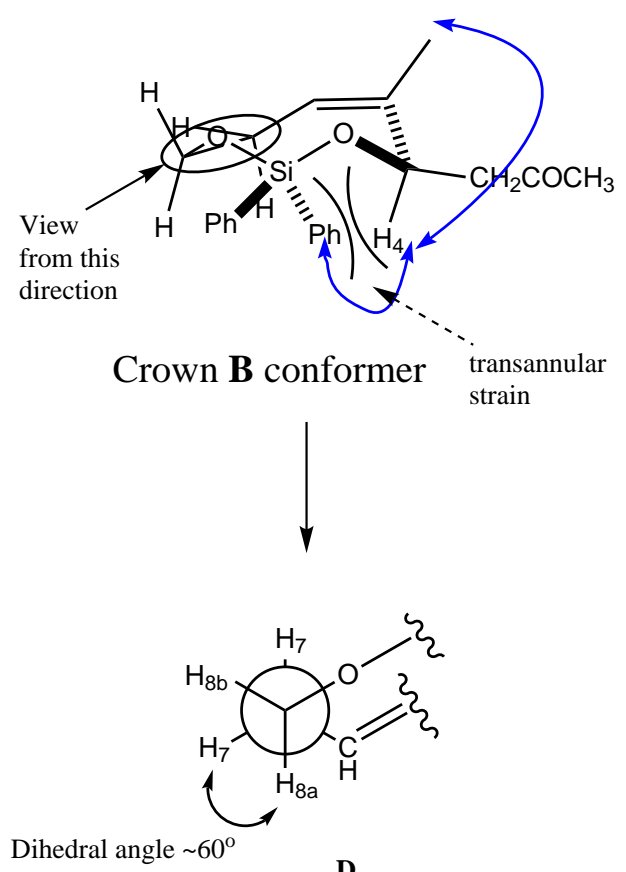

D

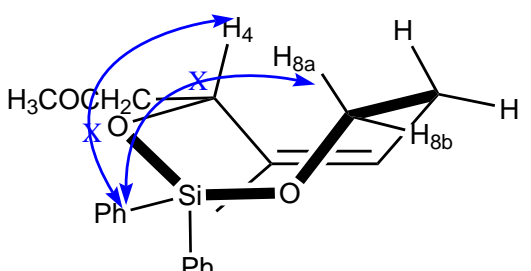

Boat conformer
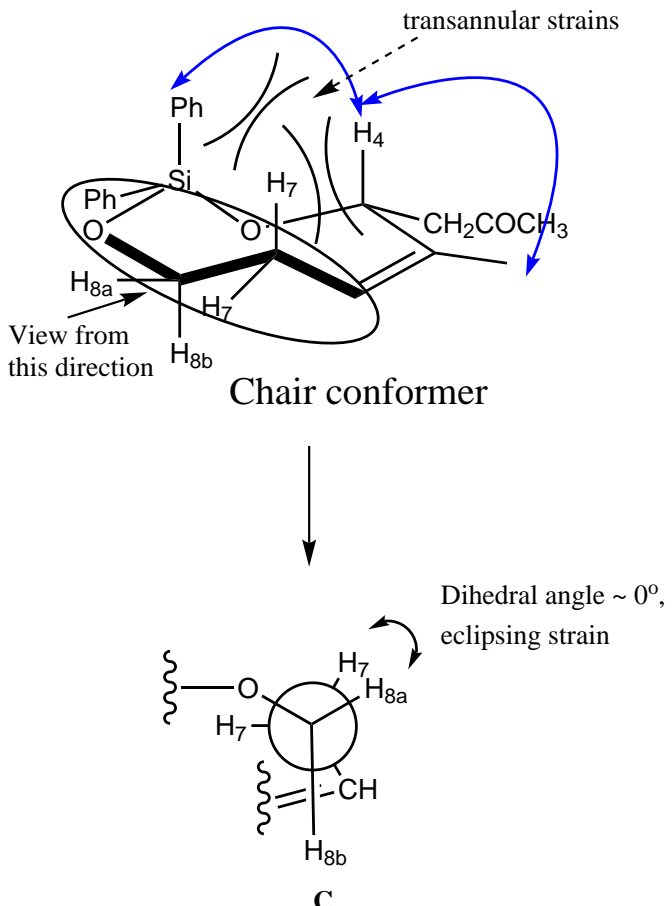

Key:

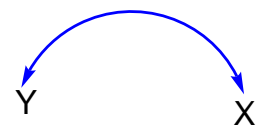

Both $X$ and $Y$ are expected to

show nOe correlations with each other

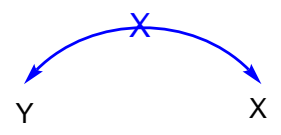

Neither $X$ nor $Y$ are expected to

show nOe correlation with the other

Figure 3.7: Possible Conformers of the Eight-Membered Ring of 2 
B-like structure as the preferred conformer. Therefore, Crown-B conformer is assumed to be the stable confomation of the eight-membered silyl acetal of $\mathbf{2}$ in solution.

When considering the side chain (from $\mathrm{C}_{1}$ to $\mathrm{C}_{4}$ ) of $\mathbf{2}$ (square box in Figure 3.8), it is interesting to note that $\mathrm{H}_{1}$ shows a correlation with both $\mathrm{H}_{3}$ and $\mathrm{H}_{4}$, suggesting that the carbon chain of $\mathrm{C}_{1}-\mathrm{C}_{4}$ does not have a staggered conformation and the electronegative oxygen prefers to be pointing away from the eight-membered ring, which presumably minimizes the overall molecular dipole. $\mathrm{H}_{3 a}$ and $\mathrm{H}_{3 b}$ have different vicinal coupling constants, $J=3.7 \mathrm{~Hz}$ and $J=$ $9.5 \mathrm{~Hz}$, respectively, which imply different dihedral angles, plausibly $60^{\circ}$ and $180^{\circ}$ to $\mathrm{H}_{4}$, respectively, thus the conformation of $\mathrm{C}_{2}-\mathrm{C}_{3}$ in Figure 3.8 was deduced. Since the combination of ${ }^{1} \mathrm{H}-{ }^{1} \mathrm{H}$ coupling constants and the nOe study had revealed the plausible stable conformers of eight-membered cyclic $\mathbf{2}$, further investigations on acyclic $\mathbf{3}$ were conducted.
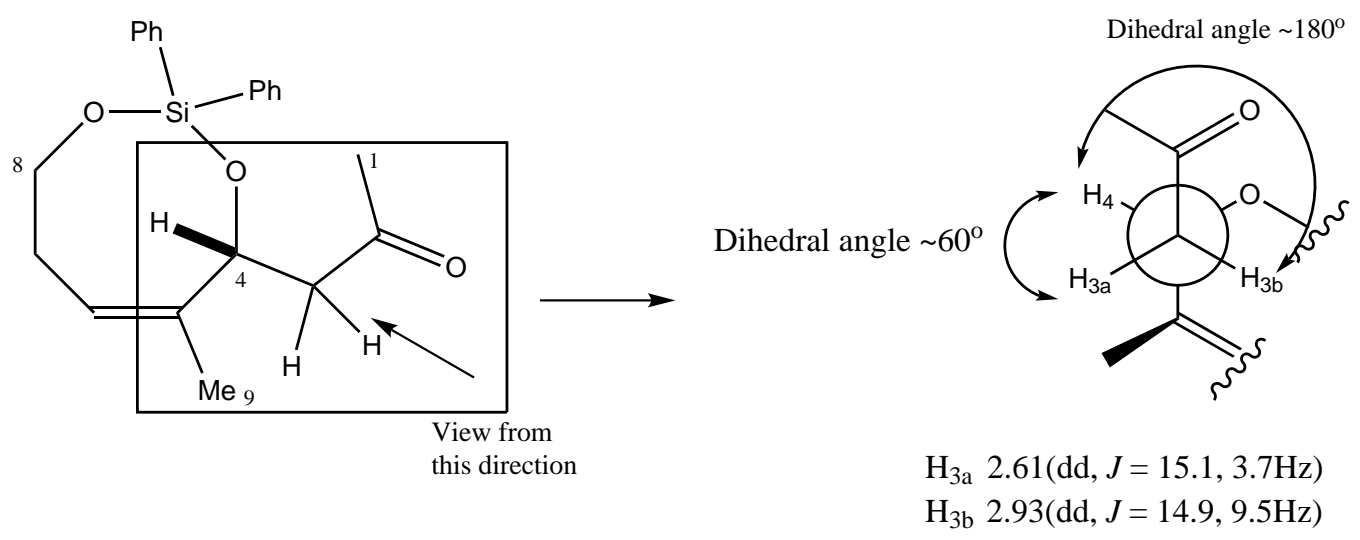

Figure 3.8: Conformation of $\mathrm{C}_{3}-\mathrm{C}_{4}$ Bond of $\mathbf{2}$

In contrast to cyclic $\mathbf{2}$, acyclic $\mathbf{3}$ shows a very different nOe correlation pattern. The key nOe correlations and the ${ }^{13} \mathrm{C}$ NMR and ${ }^{1} \mathrm{H}$ NMR spectral data are presented in Table 3.3 and more detailed nOe correlations in Figure 3.9. The phenyl protons show correlations with a variety of protons, including $\mathrm{H}_{1}, \mathrm{H}_{6}$ and $\mathrm{H}_{10}$, suggesting that both $\mathrm{Si}-\mathrm{O}$ bonds can freely rotate and the two phenyl groups are not strongly restricted into a particular conformation. The vicinal coupling constants of $\mathrm{H}_{3 a}$ and $\mathrm{H}_{3 b}(5.1$ and $7.7 \mathrm{~Hz}$, respectively) are close to the average vicinal coupling constant when the $\mathrm{C}-\mathrm{C}$ bond is freely rotating, which is typically $7 \mathrm{~Hz},{ }^{58}$ in contrast to those of $\mathrm{H}_{3 a}$ and $\mathrm{H}_{3 b}$ in 2 ( 3.7 and $9.5 \mathrm{~Hz}$, respectively). Furthermore, $\mathrm{H}_{3 b}$ correlates with the phenyl protons, therefore these results indicate that the $\mathrm{C}_{3}-\mathrm{C}_{4}$ bond can also freely rotate in the solution. Because $\mathrm{H}_{1}$ showed nOe correlations with $\mathrm{H}_{3 a}, \mathrm{H}_{3 b}, \mathrm{H}_{4}$ and phenyl protons, it is proposed that the $\mathrm{C}_{2}-\mathrm{C}_{3}$ bond can also undergo free rotation in solution. In conclusion, this demonstrated that, as expected, the acyclic $\mathbf{3}$ is much more flexible than $\mathbf{2}$. As the flexible structure of $\mathbf{3}$ might also cause its low stereoinduction, studies of the nOe correlations within its boron-complexed aldol product were of interest. Thus, TS models could be proposed to explain why such different stereoselectivities were obtained in the aldol reactions of $\mathbf{2}$ and $\mathbf{3}$. 


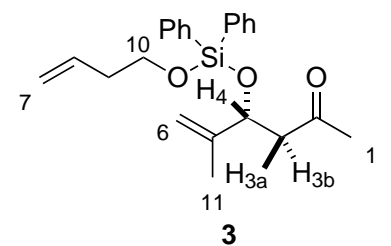

\begin{tabular}{ccll}
\hline Position & $\delta^{13} \mathrm{C}(\mathrm{ppm})$ & \multicolumn{1}{c}{$\delta^{1} \mathrm{H}(\mathrm{ppm})$} & \multicolumn{1}{c}{ Key nOe Correlations } \\
\hline 1 & 31.0 & $2.08(\mathrm{~s}, 3 \mathrm{H})$ & $\mathrm{H}_{3 a}, \mathrm{H}_{3 b}, \mathrm{H}_{4}$ and phenyl protons \\
2 & 206.7 & \multicolumn{1}{c}{-} & \\
$3 \mathrm{a}$ & 50.4 & $2.56(\mathrm{dd}, \mathrm{J}=14.9,5.1 \mathrm{~Hz}, 1 \mathrm{H})$ & $\mathrm{H}_{1}, \mathrm{H}_{4}$ and $\mathrm{H}_{11}$ \\
$3 \mathrm{~b}$ & 50.4 & $2.80(\mathrm{dd}, \mathrm{J}=14.8,7.7 \mathrm{~Hz}, 1 \mathrm{H})$ & $\mathrm{H}_{1}, \mathrm{H}_{11}$ and phenyl protons \\
4 & 73.4 & $4.78(\mathrm{~m}, 1 \mathrm{H})$ & $\mathrm{H}_{1}, \mathrm{H}_{3 a}, \mathrm{H}_{6 a}$ and phenyl protons \\
5 & 145.6 & & \\
$6 \mathrm{a}$ & 112.4 & $4.78(\mathrm{~m}, 1 \mathrm{H})$ & $\mathrm{H}_{4}$ and phenyl protons \\
$6 \mathrm{~b}$ & 112.4 & $4.90(\mathrm{~m}, 1 \mathrm{H})$ & \\
$7 \mathrm{a}$ & 116.7 & $5.07(\mathrm{app} . \mathrm{dq}, \mathrm{J}=17.1,1.6 \mathrm{~Hz}, 1 \mathrm{H})$ & \\
$7 \mathrm{~b}$ & 116.7 & $5.03(\mathrm{ddt}, \mathrm{J}=10.2,2.1,1 \mathrm{~Hz}, 1 \mathrm{H})$ & \\
8 & 135.3 & $5.82(\mathrm{ddt}, \mathrm{J}=17.1,10.3,6.8 \mathrm{~Hz}, 1 \mathrm{H})$ & \\
9 & 37.0 & $2.33(\mathrm{app} . \mathrm{qt}, \mathrm{J}=6.8,1.3 \mathrm{~Hz}, 2 \mathrm{H})$ & \\
10 & 62.8 & $3.78(\mathrm{t}, \mathrm{J}=6.8,2 \mathrm{H})$ & phenyl protons \\
11 & 17.6 & $1.71(\mathrm{~s}, 3 \mathrm{H})$ & $\mathrm{H}_{3 a}, \mathrm{H}_{3 b}, \mathrm{H}_{4}$ and phenyl protons \\
\hline
\end{tabular}

Table 3.3: ${ }^{13} \mathrm{C}$ and ${ }^{1} \mathrm{H}$ Chemical Shifts and Key nOe Correlations of $\mathbf{3}$

\subsubsection{NMR Study of the Enolization and the Aldol Reaction of 2-Hexanone}

A ${ }^{1} \mathrm{H}$ NMR study of a simple boron-mediated aldol reaction between 2-hexanone and methacrolein was undertaken as a model prior to investigation of the aldol reactions of $\mathbf{2}$ and $\mathbf{3}$. 2-Hexanone was chosen for its commercial availability and aliphatic chain, which may mimic the carbon chains of $\mathbf{2}$ and $\mathbf{3}$ without the complicating features of the protected $\beta$-hydroxy group. 2 Hexanone and its boron-containing aldol intermediates 41 and 42 (Scheme 3.23) provide suitable references to understand the NMR appearance of the conformations of the aldol intermediates and to resolve any difficulty in the NMR study of those aldol reactions. As described in Section 1.4.2 on page 29, Abiko's group published NMR studies of the bis-aldol reactions of varous acetyl compounds with a series of aldehydes and enolizations of various ketones using $\mathrm{Cy}_{2} \mathrm{BOTf}$ and $\mathrm{Et}_{3} \mathrm{~N}$. This study provides precedence for NMR analysis of boron-mediated aldol reactions at variable temperatures in $\mathrm{CDCl}_{3}$.

Modifications to Abiko's methods were investigated to accommodate the specific reagents and conditions required in the following study. For example, because an excess amount of $\mathrm{Et}_{3} \mathrm{~N}$ 

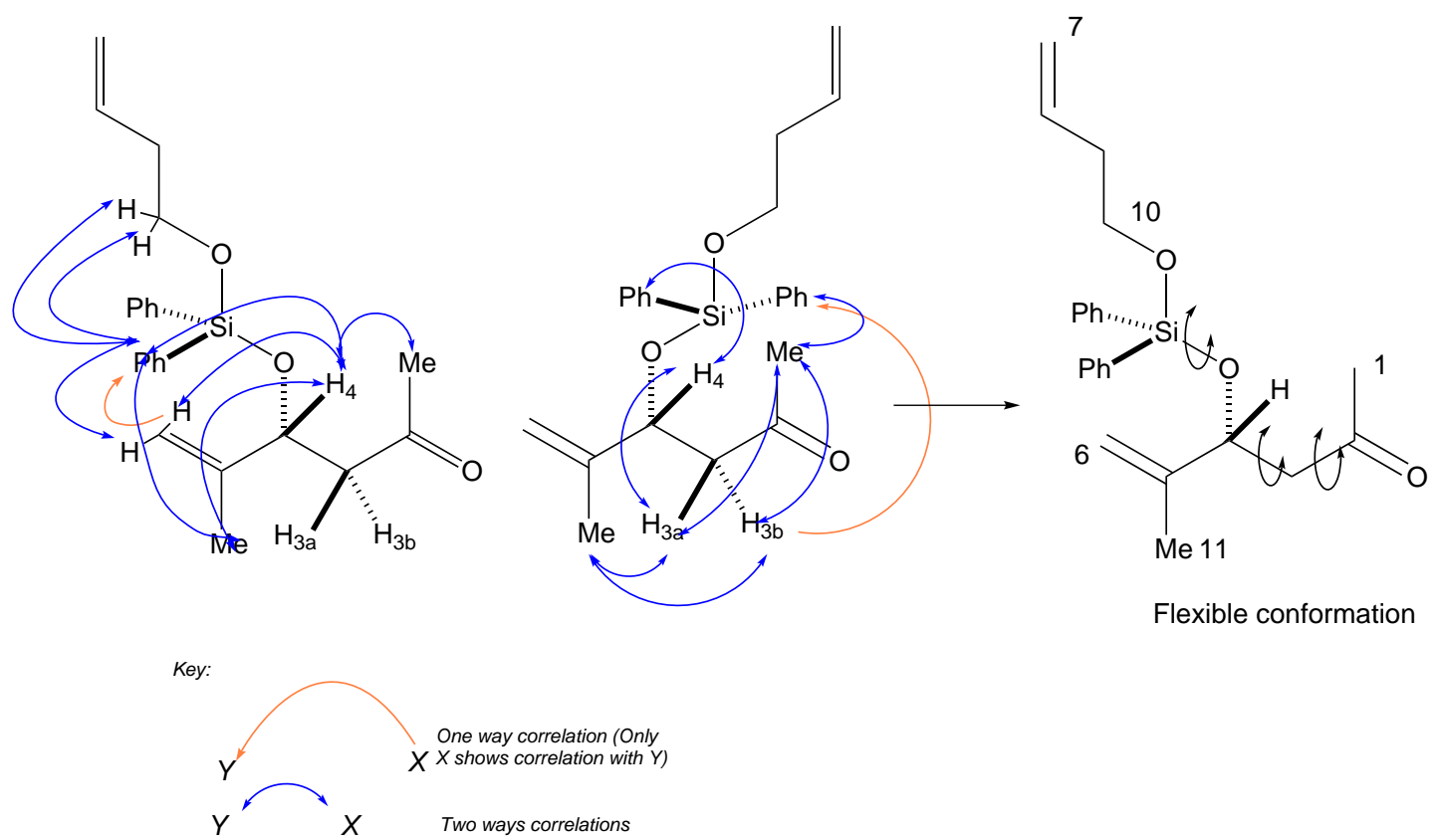

Figure 3.9: Detailed nOe Correlations of $\mathbf{3}$

is usually used in the boron-mediated aldol reaction, as was the case in this NMR study (6.0 eq.), it was discovered that the proton signals of its methylene protons swamped the region from 2.3-2.8 ppm and partially overlap with the $\mathrm{H}_{4}$ and $\mathrm{H}_{6}$ signals of $\mathbf{4 2}, \mathbf{4 3 \mathbf { b }}$ (see Scheme 3.25 on page 82 ) and 44 (see Scheme 3.26 on page 85) (discussed in the following sections). A decrease in equivalents of $\mathrm{Et}_{3} \mathrm{~N}$ (3.0 eq.) slightly improved the ability to resolve the $\mathrm{H}_{4}$ signals of 42 but it was still difficult to recognize the pattern of the coupling. Therefore, using the enolization of 2-hexanone as the model reaction, the use of different amine bases and different equivalents was investigated, thus the optimized condition could be found and applied to the substrates we were interested in. To this end, three other common amine bases, imidazole, DABCO and 2,2,6,6-tetramethylpiperidine (TMP) were used in the following studies and the results are summarized in Table 3.4. Imidazole was selected as all its protons lie in the region of aromatic protons. However, no boron enolate was detected in the ${ }^{1} \mathrm{H}$ NMR spectrum of the enolization of 2-hexanone using 1.0 eq. imidazole and 3.0 eq. $\mathrm{Cy}_{2} \mathrm{BCl}$ in $\mathrm{CDCl}_{3}$. Two more equivalents of imidazole dissolved in a minimium amount of $\mathrm{CDCl}_{3}$ were added, but still no conversion was observed. This is not unexpected since the pKa of imidazole- $\mathrm{H}^{+}$is only 6.95 which is many orders of magnitude below that of the $\alpha$-proton. 1,4-Diazobicyclo[2.2.2] octane $(\mathrm{DABCO})$ has a closer basicity to $\mathrm{Et}_{3} \mathrm{~N}$ ( $\mathrm{pKa}=8.82$ for monoprotonated DABCO, $\mathrm{pKa}=10.75$ for $\mathrm{Et}_{3} \mathrm{NH}^{+}$), thus the $\alpha$-deprotonation was successful. Because of the symmetry of DABCO, all methylene groups are homotopic to each other and the proton signals appear as a singlet at 2.77 ppm, thus the resulting ${ }^{1} \mathrm{H}$ NMR spectrum of $\mathbf{4 1}$ was less complicated. Furthermore, if only 1.0 eq. DABCO was added to this reaction, the methylene signals of the resulting monoprotonated DABCO were displayed as a multiplet from 2.96-3.09 ppm, which did not overlap with the proton signals of $\mathbf{4 1}$ at all and should not overlap with $\mathrm{H}_{4}$ and $\mathrm{H}_{6}$ of intermediates $\mathbf{4 2}, \mathbf{4 3} \mathbf{b}$ and 


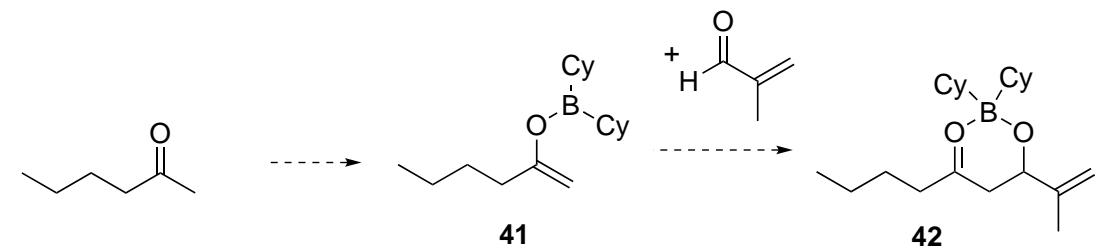

Scheme 3.23: Aldol Intermediates in the NMR study of the Aldol Reaction of 2-Hexanone<smiles>CCCCC(=O)I</smiles>

41

\begin{tabular}{|c|c|c|l|}
\hline Amine & Equivalent(s) & Success of enolization & $\begin{array}{l}\text { Critical region (2.0- } \\
\text { 3.0ppm) for structural } \\
\text { determination in }{ }^{1} \mathbf{H} \\
\text { NMR spectral data }\end{array}$ \\
\hline $\mathrm{Et}_{3} \mathrm{~N}$ & 6.0 & Yes, completion. & $\begin{array}{l}\text { Multiplet at 2.3-2.8ppm } \\
\text { blocking desired proton } \\
\text { signals }\end{array}$ \\
\hline $\mathrm{Et}_{3} \mathrm{~N}$ & 3.0 & Yes, completion. & $\begin{array}{l}\text { Multiplet at 2.3-2.8ppm } \\
\text { blocking desired proton } \\
\text { signals }\end{array}$ \\
\hline Imidazole & 1.0 & No & No blocking \\
\hline Imidazole & 3.0 & No & No blocking \\
\hline DABCO & 1.0 & Yes, completion. & No blocking \\
\hline TMP & 3.0 & Yes, completion. & No blocking \\
\hline
\end{tabular}

Table 3.4: Summary of Different Amine Bases Used in NMR Study of Enolization of 2-Hexanone

44. The enolization of 2-hexanone was also successful with TMP. Because of the absence of an $\alpha$-methylene group next to the nitrogen and the rest of its proton signals being below $2.0 \mathrm{ppm}$, the resulting ${ }^{1} \mathrm{H}$ NMR spectrum of the reaction mixture also shows a clear 2.0-3.0 ppm region which would also not overlap with the key proton signals of $42,43 \mathrm{~b}$ and 44 .

According to Abiko's study ${ }^{7}$ only an oxygen-bound mono-boron enolate was detected in the enolization of 2-butanone; thus it was expected that the enolization of 2-hexanone would behave similarly. A ${ }^{1} \mathrm{H}$ NMR spectrum of crude 41 at $-20{ }^{\circ} \mathrm{C}$ is shown in Figure 3.10. It was generated by an enolization of 2-hexanone with 3.0 eq. $\mathrm{Cy}_{2} \mathrm{BCl}$ and 1.0 eq. $\mathrm{DABCO}$ at $0{ }^{\circ} \mathrm{C}$ for $10 \mathrm{~min}$ followed by a $-20{ }^{\circ} \mathrm{C}{ }^{1} \mathrm{H}$ NMR experiment. The ${ }^{1} \mathrm{H}$ NMR experiments were also run at RT, andnearly identical spectral data was observed. The ${ }^{1} \mathrm{H}$ NMR spectrum (Figure 3.10) shows multiplets at 3.00-3.30 ppm, corresponding to the amine protons and the methylene protons of protonated DABCO. The proton signals of the cyclohexyl groups of the boron reagent were 
present as a complex multiplet below $1.9 \mathrm{ppm}$. Two sharp singlets with a 1:1 ratio of integration appeared at 4.10 and $4.21 \mathrm{ppm}$ and the ratio of the integration (1:1) remained constant in the RT ${ }^{1} \mathrm{H}$ NMR spectrum. According to Abiko's result, the formation of diborylated $(E)$ - and $(Z)$-enolates are favored by different temperatures, thus the two singlets here are not expected to correspond to the diborylated enolates. The two singlets at 4.10 and $4.21 \mathrm{ppm}$ are presumed to be the alkene protons $\mathrm{H}_{1 b}$ and $\mathrm{H}_{1 a}$ of the oxygen-bound mono-boron enolate 41, respectively, (Figure 3.11) based on the calculation method for ${ }^{1} \mathrm{H}$ chemical shifts of alkene protons described by Silverstein et al. ${ }^{58}$ The ${ }^{1} \mathrm{H}$ NMR chemical shift of $\mathrm{H}_{3}$ (broad singlet at $2.0 \mathrm{ppm}$ ) moved -0.42 ppm upfield compared to $\mathrm{H}_{3}$ of 2-hexanone (Figure 3.11), presumably due to the disappearance of the electron-withdrawing ketone in $\mathbf{4 1}$. The other proton signals of 41 were expected between 1.0 to $2.0 \mathrm{ppm}$, which overlapped with the proton signals of the boron reagents. 


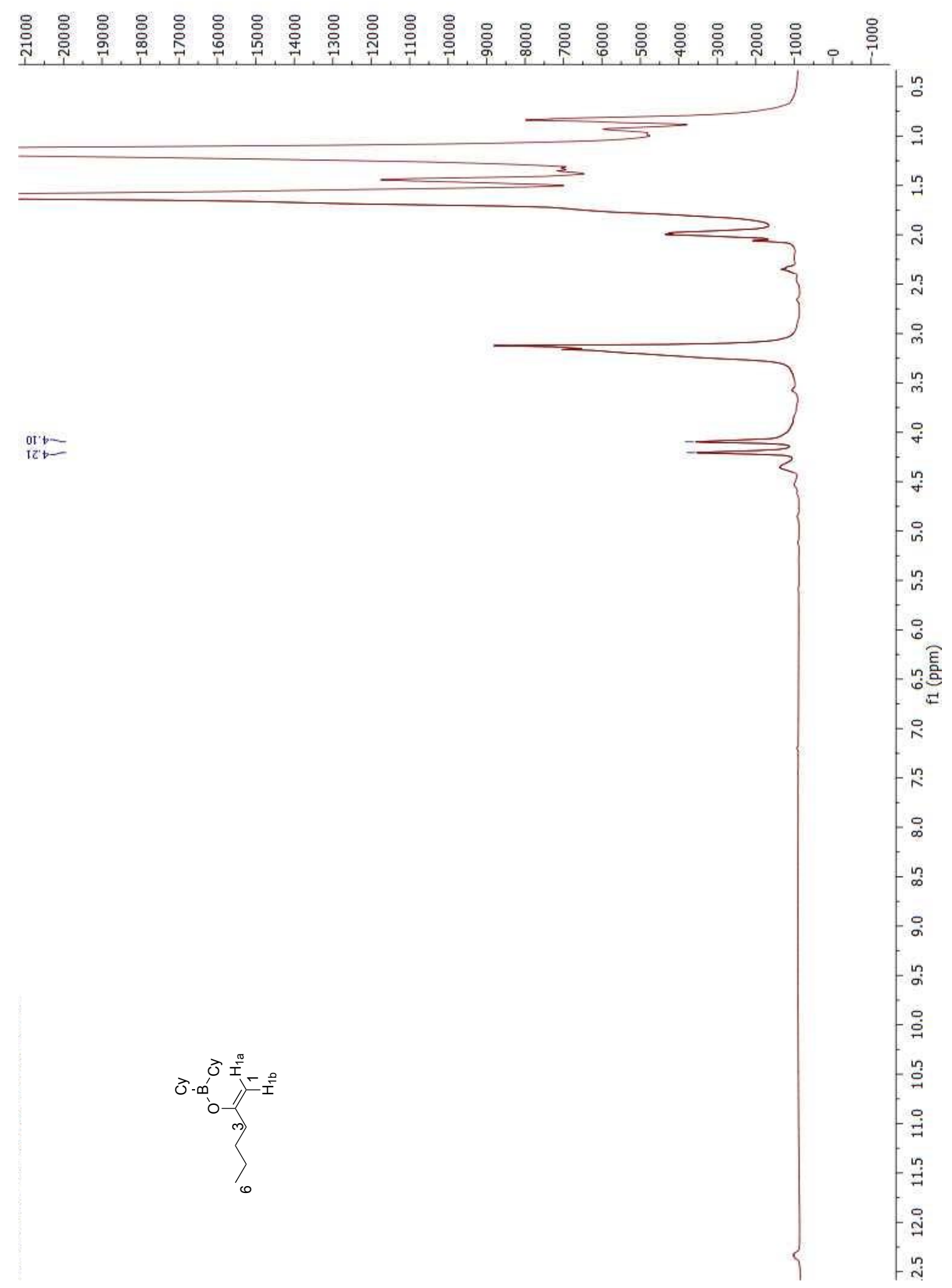

Figure 3.10: Crude ${ }^{1} \mathrm{H}$ NMR Spectrum of $\mathbf{4 1}$ (Generated at $-20{ }^{\circ} \mathrm{C}$ ) 


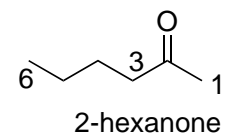

$\mathrm{H}_{1} 2.12$ (s, 3H)

$\mathrm{H}_{3} 2.42(\mathrm{t}, \mathrm{J}=8.2 \mathrm{~Hz}, 2 \mathrm{H})$

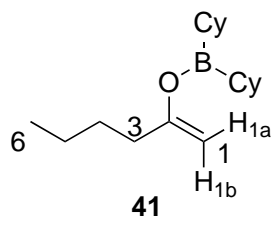

$\mathrm{H}_{1 \mathrm{a}} 4.10(\mathrm{~s}, 1 \mathrm{H})$

$\mathrm{H}_{1 \mathrm{~b}} 4.21(\mathrm{~s}, 1 \mathrm{H})$

$\mathrm{H}_{3} 2.00$ (app. s, 2H)

$\mathrm{H}_{4}-\mathrm{H}_{6}$ 1.9-0.8 (m, 7H)

Figure 3.11: Selective ${ }^{1} \mathrm{H}$ NMR Spectral Data of 2-Hexanone and $\mathbf{4 1}$

Methacrolein 27a was then added to the NMR tube at $0{ }^{\circ} \mathrm{C}$ and the ${ }^{1} \mathrm{H}$ NMR experiments were run at RT after 10 minutes. Approximately 50\% conversion of the boron enolate 41 to the boron-complexed aldol product 42 was detected in the ${ }^{1} \mathrm{H}$ NMR spectrum after the first 10 minutes but the rate of the reaction decreased quickly and only about $15 \%$ conversion happened in the following 20 minutes. Surprisingly, only very weak nOe signals were observed for product 42 even after a long period of scanning $(1 \mathrm{~h})$. This observation may be due to the quantum tumbling effect but the discussion of this effect is beyond the scope of this thesis. Fortunately, 1D ROESY experiment is commonly used to solve this problem, thus a 1D ROESY spectrum of 42 was run. The key ${ }^{1} \mathrm{H}$ chemical shifts and rOe correlations of $\mathbf{4 2}$ are shown in Figure 3.12. There is only one chiral center in $\mathbf{4 2}\left(\mathrm{C}_{3}\right)$, so it was expected that the $\mathrm{C}_{3}$ substituent prefers the equatorial position of the six-membered ring, such as in Chair $\mathbf{A}$ and Boat $\mathbf{A}$ conformers (Figure 3.13). $\mathrm{H}_{3}$ showed a strong rOe correlation with the protons of the cyclohexyl group, suggesting a 1,3-diaxial relationship. Therefore, this indicates that the Chair $\mathbf{A}$ conformer is one of the conformers of 42 in the solution. However, both $\mathrm{H}_{4 a}$ and $\mathrm{H}_{4 b}$ also showed rOe correlations with cyclohexyl groups. Although the axial cyclohexyl group might be large enough to show a rOe interaction with the equatorial $\mathrm{H}_{4 b}$ in the Chair $\mathbf{A}$ conformer, axial $\mathrm{H}_{4 a}$ was not expected to correlate with the equatorial cyclohexyl group. Furthermore, according to Goodman's calculation as shown in Figure 1.6 (page 23), the boat and the chair TSs of the boron-mediated aldol reaction of acetone with acetaldehyde had similar stabilities, thus indicating that both conformers of the aldol product may co-exist or interconvert in solution. Since 42 resembles the aldol product used in Goodman's calculation, $\mathbf{4 2}$ should also follow a similar trend. Boat $\mathbf{A}$ is the most likely conformer responsible for the rOe correlation between $\mathrm{H}_{4 a}$ and the cyclohexyl group. Chair $\mathbf{B}$ and Boat $\mathbf{B}$ conformers can also explain these rOe correlations but significant population of them is deemed unlikely because $R_{1}$ is in the axial position in these conformers and the large vicinal coupling constant of $\mathrm{H}_{4 a}$ in the ${ }^{1} \mathrm{H}$ NMR spectrum (Figure 3.12) does not match with the orientation of $\mathrm{H}_{4 a}$ in these conformers. Interestingly, based on the ${ }^{1} \mathrm{H}$ NMR spectrum, only one set of proton signals was observed, therefore we assume the rate of interconversion between Chair $\mathbf{A}$ and Boat $\mathbf{A}$ conformers is faster than the relaxation time of the excited proton. Thus, both conformers were contributing to the rOe correlation data. 


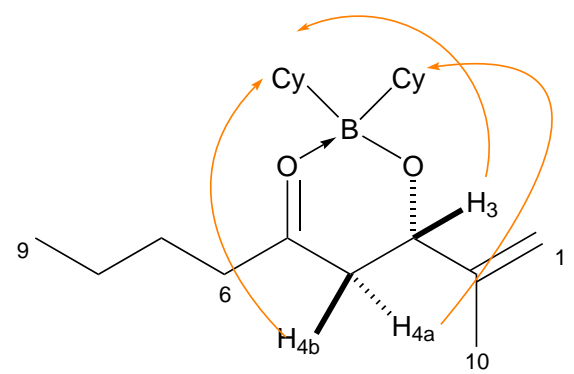

42

$\mathrm{H}_{3} 4.96$ (app. d, J = 6.46 Hz, $1 \mathrm{H}$ )

$\mathrm{H}_{4 \mathrm{a}} 2.71(\mathrm{dd}, \mathrm{J}=15.1,9.5 \mathrm{~Hz}, 1 \mathrm{H})$

$\mathrm{H}_{4 \mathrm{~b}} 2.40(\mathrm{dd}, \mathrm{J}=15.3,2.9 \mathrm{~Hz}, 1 \mathrm{H})$

Key:

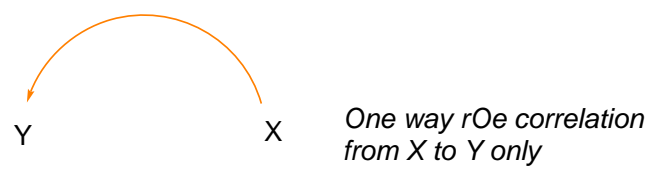

Figure 3.12: Key rOe Correlations of $\mathbf{4 2}$

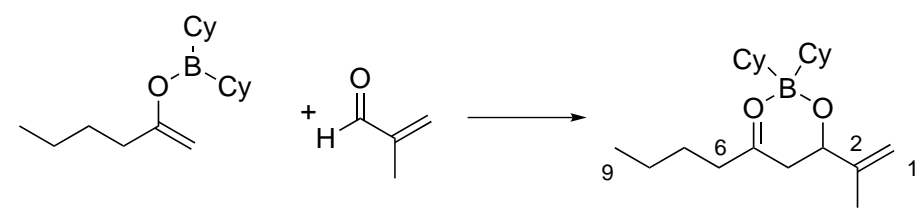

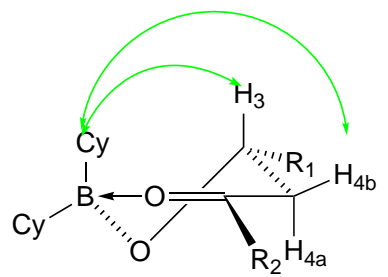

Chair A conformer

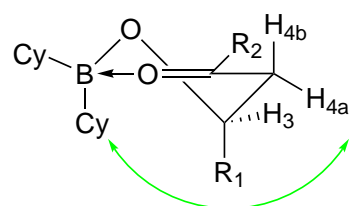

Chair B conformer

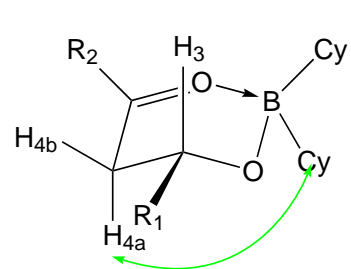

Boat A conformer

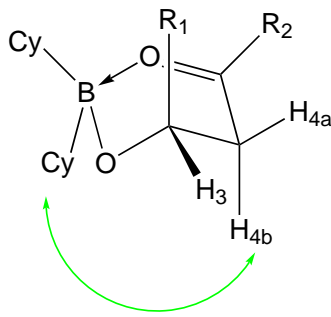

Boat B conformer

Key:

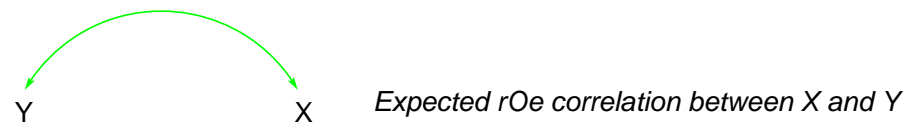

$R_{2}=\left(\mathrm{CH}_{2}\right)_{3} \mathrm{CH}_{3}$

Figure 3.13: Possible Conformers for $\mathbf{4 2}$ 
The larger vicinal coupling constant of $\mathrm{H}_{4 a}$ in $\mathbf{4 2}$ is due to its anti configuration to $\mathrm{H}_{3}$ and can only be observed when $\mathrm{H}_{3}$ and $\mathrm{R}_{1}$ are sitting at axial and equatorial positions, respectively, as in Chair $\mathbf{A}$ and Boat $\mathbf{A}$ conformers (Figure 3.13). Therefore by looking at the vicinal coupling constants of their $\mathrm{H}_{4 a}$ and $\mathrm{H}_{4 b}$ and the rOe correlation patterns, the conformations of the aldol products of $\mathbf{2}$ and $\mathbf{3}$ in solution may be assigned.

\subsubsection{NMR Studies of Aldol Reactions of 2 and 3 NMR Investigations of Boron Enolates of 2 and 3}

Unlike the enolization of 2-hexanone, diborylation of $\mathbf{2}$ is plausible since the presence of a $\beta$-oxygen could stabilize the carbon-bound boron-substituent. A modification of Abiko's mechanism for explaining the 1,5-anti stereoinduction is shown in Scheme 3.24. ${ }^{7}$ A mono-O-bound boron enolate 46 a could be formed first, followed by a rearrangement to afford a mono-C-bound ketone $46 \mathrm{~b}$. The $\beta$-oxygen can "lock" the boron reagent of $46 \mathrm{~b}$ by chelation to form $46 \mathrm{c}$. Thus the carbonyl oxygen is available again for a second borylation and enolized to form the diborylated enolate $\mathbf{4 7 a}$ and/or $\mathbf{4 7} \mathbf{b}$. $\mathbf{4 7 a}$ and $\mathbf{4 7} \mathbf{b}$ structurally resemble $(E)$ - and $(Z)$-internal enolates, thus potential Zimmerman-Traxler TSs can be proposed for the following aldol reaction. The carbon-bound boron-substituent of $\mathbf{4 7} \mathbf{a}$ is stabilized by the $\beta$-alkoxy substituent, thus the bicyclic TSs of $\mathbf{4 7 a}, \mathbf{A}$ and $\mathbf{B}$, are expected to be favored over $\mathbf{C}, \mathbf{D}$ and $\mathbf{E}$ of $\mathbf{4 7} \mathbf{b}$. In TS $\mathbf{B}$, the $\mathrm{R}$ group of $\mathbf{4 7 a}$ points towards the six-membered chair and interacts unfavorably with the axial substituent of the oxygen bound boron reagent and the formyl hydrogen of the aldehyde. In contrast, the $\beta$-hydrogen of $\mathbf{4 7 a}$ points towards the six-membered chair in TS $\mathbf{A}$, which has much less steric interference and leads to a 1,5-anti aldol product. To test the validity of this mechanism, an NMR investigation of the enolization of $\mathbf{2}$ was carried out. 


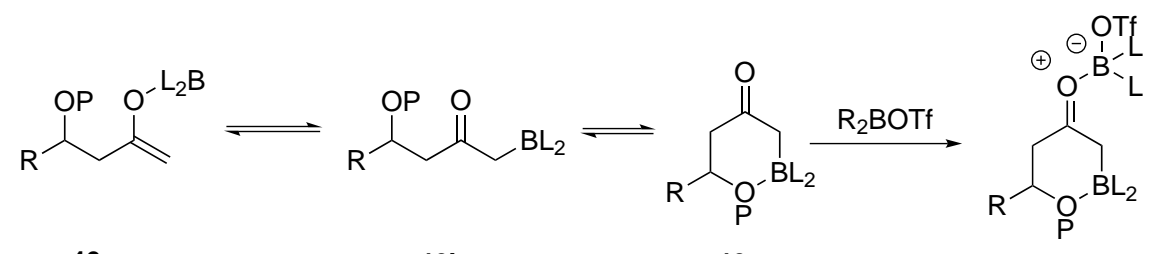

$46 a$

$46 b$

$$
\text { 46c }
$$

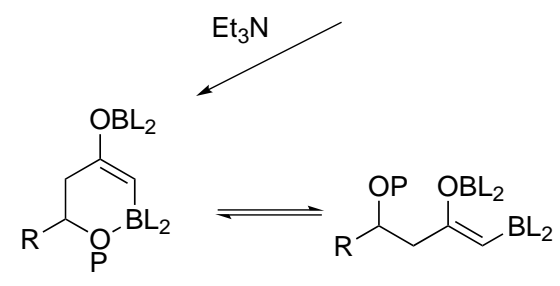

47a

47b

1,5-anti product from

TS A (major product)

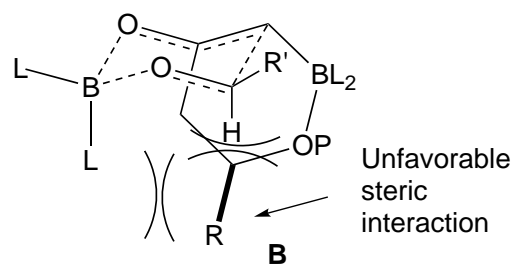

$\mathrm{P}=$ Protecting group

A

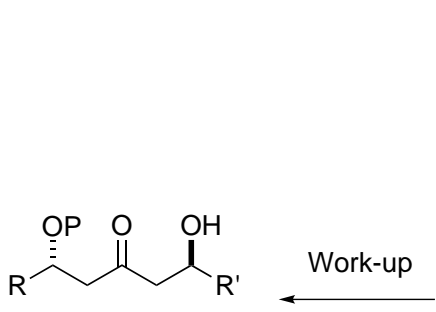

$$
\downarrow+\mathrm{R}^{\prime} \mathrm{CHO}
$$<smiles>C[12CH2]C=[18O]</smiles>
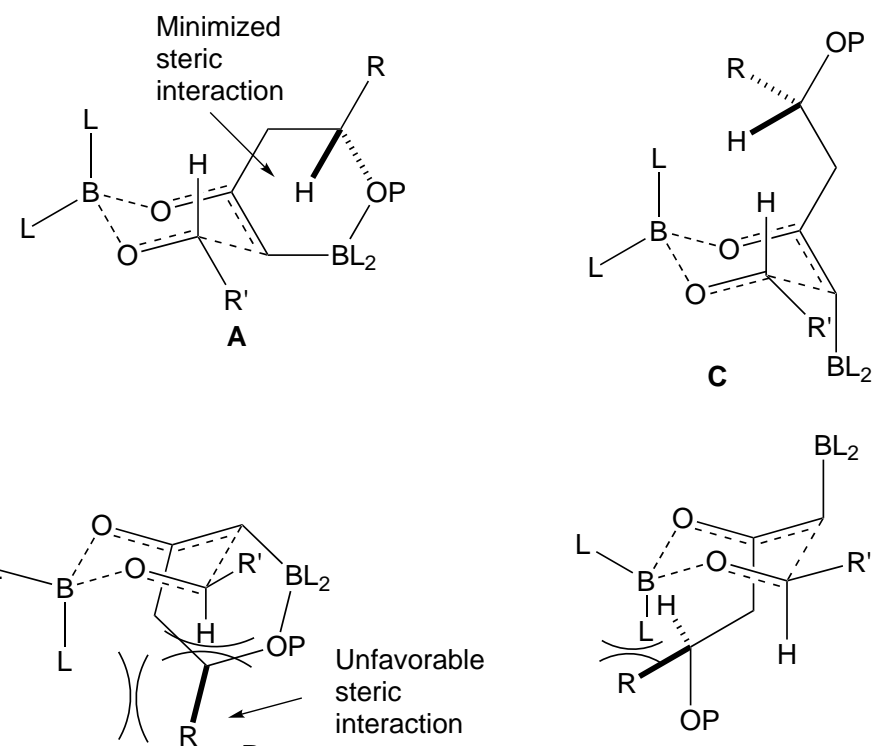

D

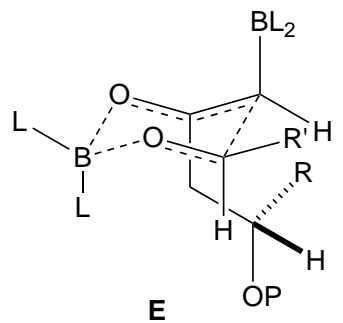

Scheme 3.24: Proposed Mechanism of Aldol Reactions of Diborylated Enolates Derived from $\beta$-Oxygenated Methyl Alkyl Ketones

The enolization of cyclic silyl acetal 2 was undertaken with 3.0 eq. $\mathrm{Cy}_{2} \mathrm{BCl}$ and 3.0 eq. TMP at $0{ }^{\circ} \mathrm{C}$. After 10 minutes, ${ }^{1} \mathrm{H}$ NMR spectra of the resulting boron-containing intermediate were obtained at $-20{ }^{\circ} \mathrm{C}$ and then at RT. Both showed a very similar pattern. The crude ${ }^{1} \mathrm{H}$ NMR spectrum obtained at $-20^{\circ} \mathrm{C}$ is shown in Figure 3.15 (page 79). The broad singlet at $8.75 \mathrm{ppm}$ is the amine proton of TMP and the rest of the protons of TMP are below $2.0 \mathrm{ppm}$, covered by 
a complex multiplet corresponding to the protons of $\mathrm{Cy}_{2} \mathrm{BCl}$. The key proton signals of $\mathbf{4 8}$ are summarized and shown in Figure 3.14.
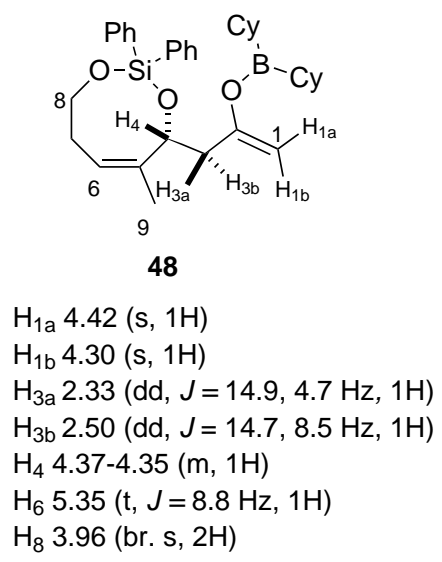

Figure 3.14: Key ${ }^{1} \mathrm{H}$ Spectral Data of $\mathbf{4 8}$

As described by Abiko, ${ }^{7}$ the ratio of $(E)$ - and $(Z)$-diborylated enolates changes significantly at different temperatures (Scheme 1.17 on page 32). However, as shown in Figure 3.15, the two singlets at 4.42 and $4.30 \mathrm{ppm}$ had ca. 1:1 ratio of integration at both $-20{ }^{\circ} \mathrm{C}$ and $\mathrm{RT}$, thus they are not thought to belong to diborylated enolates, presumably instead correponding to $\mathrm{H}_{1 a}$ and $\mathrm{H}_{1 b}$ of $\mathbf{4 8}$, respectively (according to the calculation guide reported by Silverstein et al. ${ }^{58}$ ). Thus, only the oxygen bound mono-borylated terminal enolate 48 was detected in this enolization (Figure 3.14) and so a modified Abiko's mechanism is not relevant to the 1,5-antistereoinduction of 2 .

Considering Hoberg's model, ${ }^{4}$ the $\pi$-stacking between the phenyl ring and enolate should happen immediately after enolization. In response to the anisotropic effect from the phenyl ring, the enolate protons, such as $\mathrm{H}_{1 a}$ and $\mathrm{H}_{1 b}$ of $\mathbf{4 8}$, should shift upfield from those of $\mathbf{4 1}$, the boron enolate of 2 -hexanone. Conversely, the presence of the $\beta$-diphenylsiloxy group may cause downfield-shifting effects by inductively withdrawing the electron density from the protons. As evidence of this, a comparison of the ${ }^{1} \mathrm{H}$ spectral data of $49 \mathrm{a}$ and $49 \mathrm{~b}$ shows a $+0.15 \mathrm{ppm}$ (downfield) shift in the terminal alkyl protons' chemical shift $\left(\mathrm{H}_{4}\right.$ at $\delta$-carbon) as a result of $\beta$-diphenylsiloxy group of $\mathbf{4 9 b}$ (Figure 3.16). ${ }^{59,60} \mathrm{~A}$ similar dowfield-shifting effect is observed for the terminal alkene protons at the $\delta$-carbon to the diphenyl silyloxy group $(+0.07-+0.09 \mathrm{ppm})$ of 3 compared to those of 1-pentene 49c. Bringing these together, the inductively deshielding effect of a $\beta$-diphenylsiloxy group to the protons at the $\delta$-carbon is in the range of $+0.07-+0.15$ ppm which is not as strong as the upfield-shift caused by the $\pi$-stacking. There are two good examples for the large upfield-shifts of the alkene protons caused by the anisotropic effect from the phenyl ring during $\pi$-stacking, reported by Corey ${ }^{61}$ and Riche ${ }^{62,63}$ and shown in Table 3.5 (page 80 ) and 3.6 (page 80). The $\pi$-stacking between the phenyl ring and the 2-butenate in $\mathbf{5 0 b}$ (Entry 2 in Table 3.5) causes an upfield-shift of approximately $-0.5 \mathrm{ppm}$ for both alkene protons 
relative to 50a. Interestingly, the protons of the methyl group of $\mathbf{5 0 b}$ which are one carbon further removed from the center of the $\pi$-stacking, also experienced small upfield-shifting effects (-0.06 ppm). Thus this indicates the "shielding" cone of the phenyl ring in this $\pi$-stacking covers a large area. A similar trend is observed with 8-phenylmenthyl ester 50e where shifts from -0.25 to $-0.54 \mathrm{ppm}$ upfield compared to methyl ester 50c were observed. Again, depending on the location of the proton in the alkene, the magnitude of the upfield-shifting effect varied. Interestingly, Riche also discovered that a conformationally restricted six-membered ring is optimal for a full $\pi$-stacking to operate (Entries 2 and 3 in Table 3.6) and that the ${ }^{1} \mathrm{H}$ NMR chemical shifts of the corresponding alkene protons are good indicators of $\pi$-stacking. Therefore, the anisotropic effect of the phenyl ring on the chemical shifts of the alkene protons are expected to be more dominant than the inductive electron-withdrawing effect from the $\beta$-diphenylsiloxy group if they exist in 48. Although the strength of the anisotropic effect from the phenyl ring depends on the relative positioning of the enolate protons and the phenyl ring, it is very unlikely that neither enolate proton experience strong upfield-shifting effects because they are right next to the center of the $\pi$-stacking and at $120^{\circ}$ to each other. Thus, at least one proton of the enolate would experience a strong anisotropic effect from the phenyl ring. 


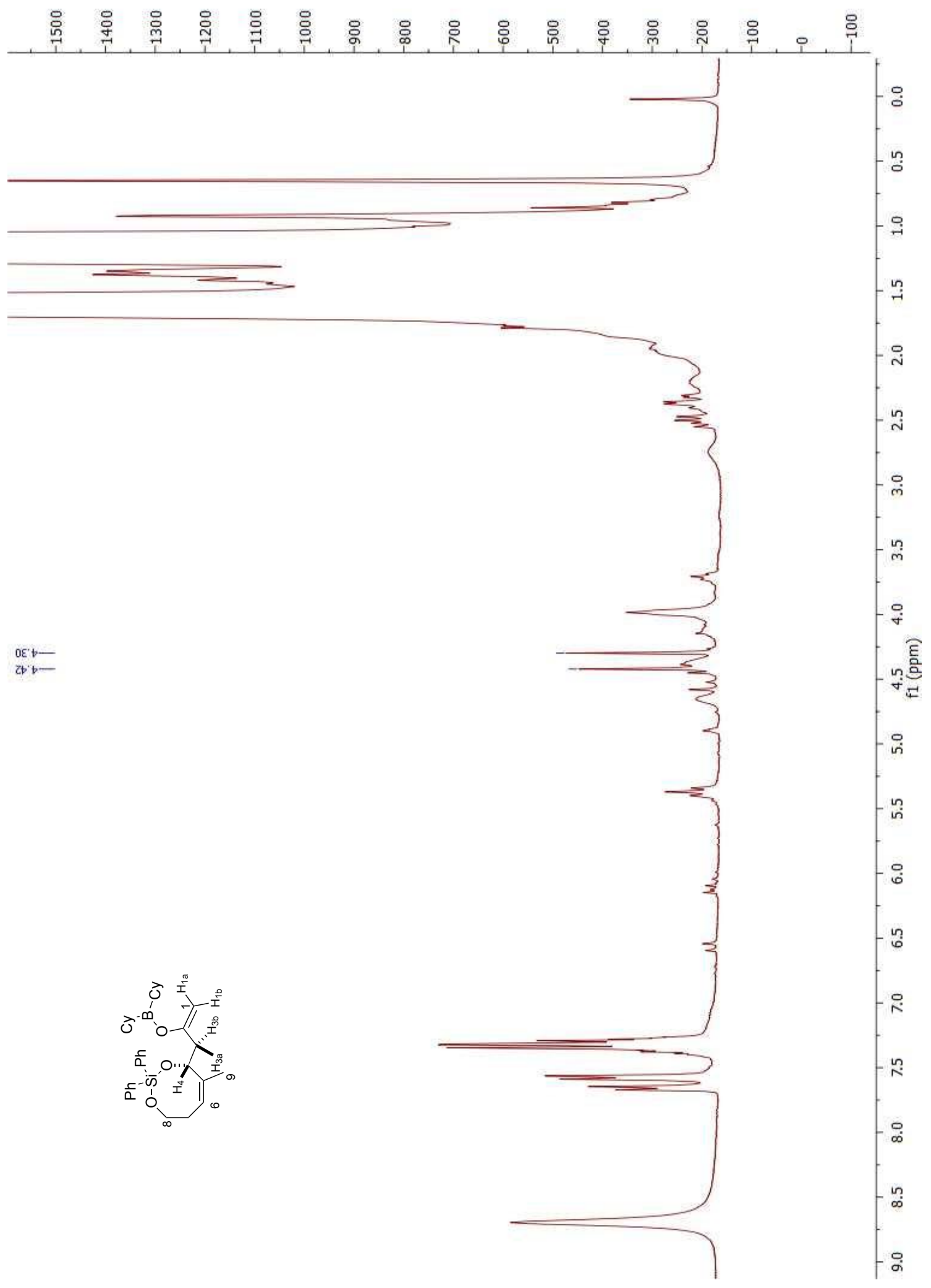

Figure 3.15: Crude ${ }^{1} \mathrm{H}$ NMR Spectrum of 48 (Generated at $-20{ }^{\circ} \mathrm{C}$ ) 


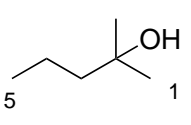

$49 a$

$\mathrm{H}_{1} 1.20(\mathrm{~s}, 6 \mathrm{H})$

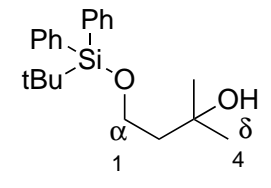

$49 b$<smiles>CC=CCCC</smiles>

$49 c$<smiles>C=C(C)C(CC(=O)I)O[Si](OCC=CC)(c1ccccc1)c1ccccc1</smiles>

3

$\mathrm{H}_{1 \mathrm{a}} 4.96-4.93(\mathrm{~m}, 1 \mathrm{H}) \quad \mathrm{H}_{7 \mathrm{a}} 5.04-5.02(\mathrm{~m}, 1 \mathrm{H})$

$\mathrm{H}_{1 \mathrm{~b}} 5.03-4.96(\mathrm{~m}, 1 \mathrm{H}) \quad \mathrm{H}_{7 \mathrm{~b}} 5.09-5.05(\mathrm{~m}, 1 \mathrm{H})$

Figure 3.16: Downfield-Shifting Effects from the $\beta$-Diphenylsiloxy Groups $\left({ }^{1} \mathrm{H}\right.$ NMR Spectral Data Obtained in $\mathrm{CDCl}_{3}$ )

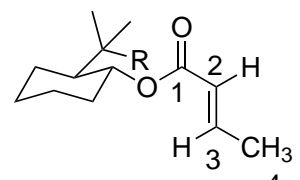

\begin{tabular}{cccccc}
\hline Entry & Substrate & $\mathrm{R}$ & $\mathrm{H}_{2}(\mathrm{ppm})$ & $\mathrm{H}_{3}(\mathrm{ppm})$ & $\mathrm{H}_{4}(\mathrm{ppm})$ \\
\hline 1 & $\mathbf{5 0 a}$ & $\mathrm{H}$ & 5.83 & 6.96 & 1.78 \\
2 & $\mathbf{5 0 b}$ & $\mathrm{Ph}$ & 5.32 & 6.44 & 1.72 \\
\hline
\end{tabular}

Table 3.5: Upfield-Shifting Effect from the Anisotropic Effect of the Phenyl Ring

\begin{tabular}{cccccc}
\multicolumn{6}{c}{} \\
\multicolumn{7}{c}{} \\
\hline Entry & Substrate & $\mathrm{R}$ & $\mathrm{H}_{2}(\mathrm{ppm})$ & $\mathrm{H}_{3 a}(\mathrm{ppm})$ & $\mathrm{H}_{3 b}(\mathrm{ppm})$ \\
\hline 1 & $\mathbf{5 0 c}$ & methyl & 6.13 & 6.40 & 5.82 \\
2 & $\mathbf{5 0 d}$ & 3 -phenylpropyl & 6.10 & 6.39 & 5.77 \\
3 & $\mathbf{5 0 e}$ & $(+)-8$-phenylmenthyl & 5.59 & 6.01 & 5.57 \\
\hline
\end{tabular}

Table 3.6: Different Upfield-Shifting Effects From Flexible to Conformationally Restricted Phenyl Groups for Acryloyl Esters

If there is $\pi$-stacking between the phenyl group and the enolate of $\mathbf{4 8}, \mathrm{H}_{1 a}$ and $\mathrm{H}_{1 b}$ would still shift upfield by about -0.10 to $-0.39 \mathrm{ppm}$ compared to $\mathrm{H}_{1 a}$ and $\mathrm{H}_{1 b}$ of $\mathbf{4 1}$, taking the deshielding effect from $\beta$-diphenylsiloxy group into consideration. However, if no $\pi$-stacking occurs, the deshielding effect from the $\beta$-oxygen would become dominant, and +0.07 to +0.15 ppm downfield-shift would be expected. After comparing ${ }^{1} \mathrm{H}$ spectra of $\mathbf{4 1}$ and $\mathbf{4 8}$, downfield shifts of ca. +0.20 ppm for $\mathrm{H}_{1 a}$ and $\mathrm{H}_{1 b}$ of $\mathbf{4 8}$ were observed (indicated in Figure 3.17), which indicates that the enolate protons are not subject to an anisotropic effect, which suggests that no significant $\pi$ - $\pi$ interaction between the phenyl ring and the enol in $\mathbf{4 8}$. 


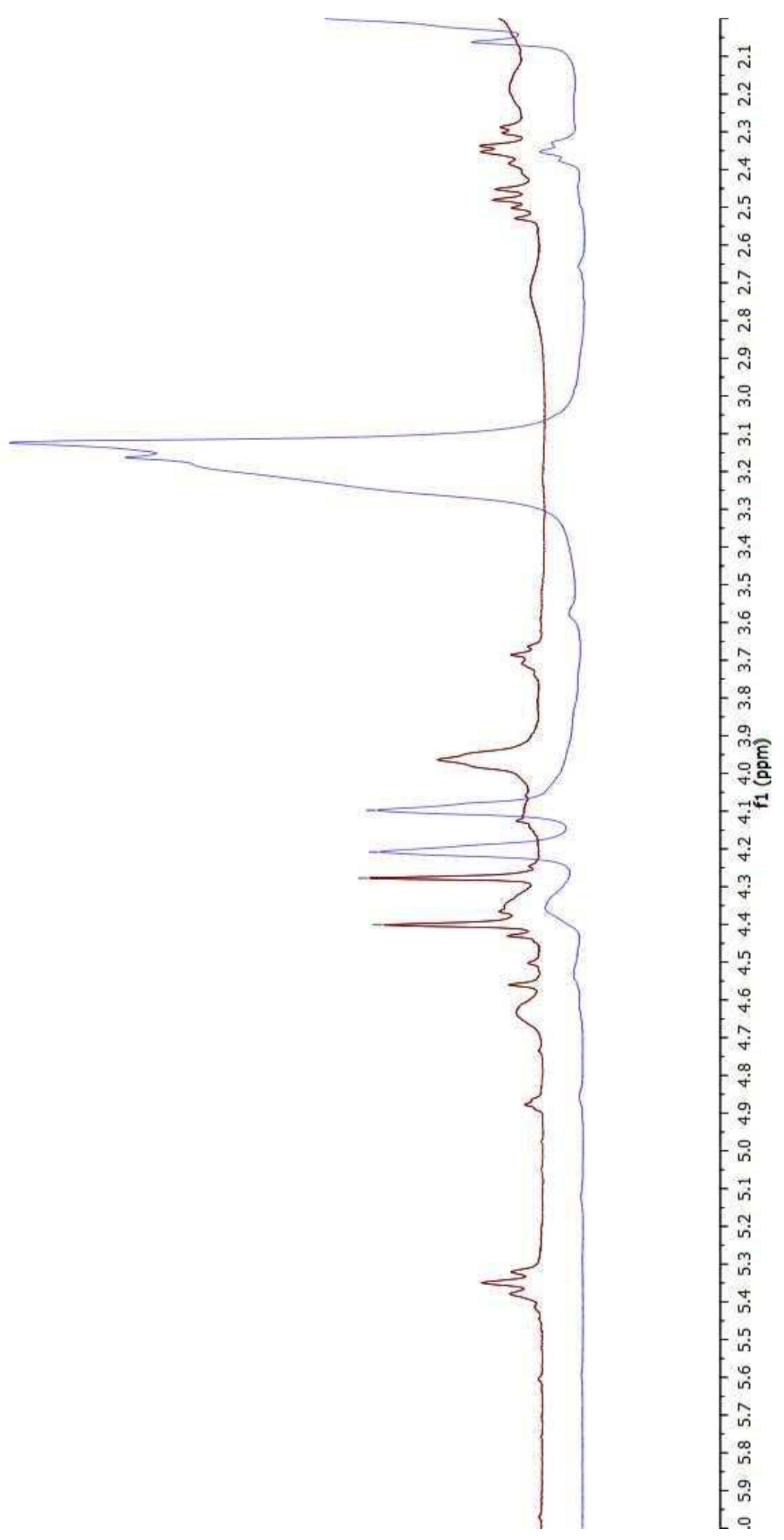

Figure 3.17: Stacked ${ }^{1} \mathrm{H}$ NMR Spectra of $\mathbf{4 1}$ (Blue) and $\mathbf{4 8}$ (Red) 


\section{NMR Investigations of Aldol Product Chelating Boron Complexes and Goodman's Model}

In contrast to Hoberg's model, Goodman's model for explaining the stereochemical outcome from 1,5-anti aldol reactions is based on the TS. However, since it is impossible to trap a TS on the timescale of ${ }^{1} \mathrm{H}$ NMR, we can only extract the relevant information from the conformations of 43a and 43b (Scheme 3.25) and thereby infer a model for the TS. The addition of $\mathbf{2 7 a}$ to 48 was done at $0{ }^{\circ} \mathrm{C}$ (Scheme 3.25). The ${ }^{1} \mathrm{H}$ NMR experiments were run at $\mathrm{RT}$ and then $-20{ }^{\circ} \mathrm{C}$ with 500 and $300 \mathrm{MHz}$ instruments, respectively, immediately after the addition of methacrolein; COSY (with 300 and $500 \mathrm{MHz}$ instruments) and 1D ROESY (with $300 \mathrm{MHz}$ instrument) experiments were run at RT one day after the addition. As expected, the proton signals of $\mathbf{4 3} \mathbf{b}$ built up at a similar rate to the decrease of those of the boron enolate 48. However, 43a was not detected in the ${ }^{1} \mathrm{H}$ NMR spectra. This presents two technical challenges to the use of NMR in this investigation:

- Firstly, because only $43 \mathrm{~b}$ was detected in the ${ }^{1} \mathrm{H}$ NMR spectrum, this implies that the conversion from $\mathbf{4 3 a}$ to $\mathbf{4 3} \mathrm{b}$ is an exothermic process. According to Hammond's postulate, the TS of an exothermic reaction resembles the reactant, thus $\mathbf{4 3 a}$ is the better substrate for investigating the conformation of the TS but only $43 \mathrm{~b}$ is available.

- Secondly, one of the reasons that the boat TS becomes dominating over chair ones in Goodman's calculation is the presence of a proposed hydrogen bond between the $\beta$-oxygen and the formyl hydrogen of the aldehyde (Section 1.4.2 on page 26). The electron-withdrawing carbonyl group causes the acidity and therefore the hydrogen bonding ability of the formyl hydrogen. However, in the aldol product $43 \mathrm{~b}$, an $\mathrm{sp}^{3}$-hybridized $\mathrm{C}_{3}-\mathrm{H}_{3}$ bond is present instead, in which the proton is far less acidic; indeed, the $\alpha$-protons $\mathrm{H}_{4}$ next to the ketone are expected to be more acidic than $\mathrm{H}_{3}$. Therefore Goodman's proposed hydrogen bond is likely to be lost in $\mathbf{4 3 b}$.

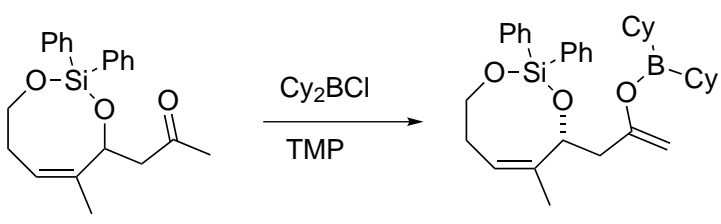

2
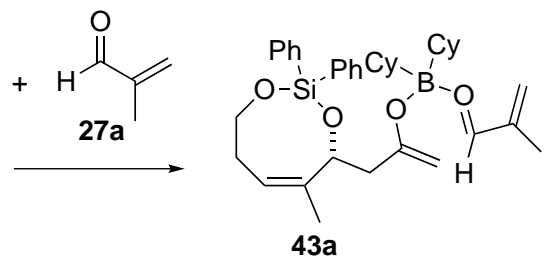

48

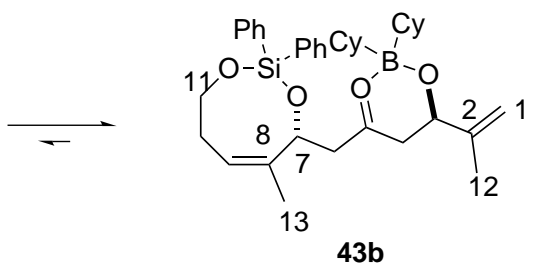

Scheme 3.25: Aldol Reaction of $\mathbf{2}$ with $\mathbf{2 7 a}$ in the NMR Study 
Nevertheless, COSY and 1D ROESY experiments of $\mathbf{4 3 b}$ were also run at RT and the key rOe correlation is shown in Figure 3.18 and detailed ${ }^{1} \mathrm{H}$ spectral data are shown in Table 3.7. Within the six-membered ring of $\mathbf{4 3 b}, \mathrm{C}_{3}$ is the only chiral center, thus it was assumed that the non-hydrogen substituent of $\mathrm{C}_{3}$ is in an equatorial position ( $\mathrm{R}$ group in Figure 3.18 ) in the following discussion. Using the COSY spectrum, we found out that one $\mathrm{H}_{6}$ overlaps with one $\mathrm{H}_{4}$ at $2.55 \mathrm{ppm}$ to form two merging dd signals. Both dd show small vicinal coupling constants (2.9-3.0 Hz), implying the dihedral angles between these protons and the corresponding vicinal protons are small and syn relationships are present. These protons are assigned to $\mathrm{H}_{6 a}$ and $\mathrm{H}_{4 b}$ of $43 \mathrm{~b}$ in Figure 3.18. It was found that $\mathrm{H}_{6 b}$ and $\mathrm{H}_{4 a}$ also overlap at 2.81ppm (based on the COSY spectrum) but the coupling constant of $\mathrm{H}_{4 a}$ was very hard to interpret. With this information in hand, the rOe correlations of $43 \mathrm{~b}$ became more clear. Although there were significant TOCSY signals found between 1.2 and 1.6ppm in the 1D ROESY spectra, with careful analysis it is clear that only the multiplet containing $\mathrm{H}_{4 a}$ and $\mathrm{H}_{6 b}$ showed an rOe correlation with the proton(s) of the cyclohexyl group at $1.0 \mathrm{ppm}$, but selective irradiation of the individual signals of $\mathrm{H}_{4 a}$ and $\mathrm{H}_{6 b}$ was not successful. Based on the positions of these two protons, this correlation is more likely attributed to $\mathrm{H}_{4 a}$ and the cyclohexyl group but no absolute conclusion can be made at this stage. No 1,3-rOe-correlation between the cyclohexyl group and $\mathrm{H}_{3}$ was detected in $\mathbf{4 3 \mathbf { b }}$, which contradicts the expectation shown in chair conformer in Figure 3.18, thus suggesting that the six-membered chair might not be the most prevalent conformer. However, the absence of a nOe or rOe correlating signal may not be significant. For example, a lack of correlation between two close protons could occur due to an insufficient average time for them to be close in space. Therefore, we decided to run the $1 \mathrm{D}$ ROESY experiment at $-20^{\circ} \mathrm{C}$. If the chair conformer of $\mathbf{4 3 b}$ was the more stable one, it should become dominant at low temperature and the average time for $\mathrm{H}_{3}$ and a cyclohexyl group to be close in space should also be longer. Although the resolution of the $300 \mathrm{MHz}{ }^{1} \mathrm{H}$ NMR spectrum at $-20{ }^{\circ} \mathrm{C}$ was not as good as that from the $500 \mathrm{MHz}$ instrument at $\mathrm{RT}$, the positions of the protons in the spectrum were roughly constant. Interestingly, the rOe correlation pattern of $43 \mathrm{~b}$ at $-20{ }^{\circ} \mathrm{C}$ was very similar to the one at $\mathrm{RT}$ and only the multiplet of $\mathrm{H}_{4 a}$ and $\mathrm{H}_{6 b}$ showed a correlation with the proton(s) of a cyclohexyl group at 1.0ppm. This result again suggested the chair conformer is not the most stable one for $\mathbf{4 3 b}$ (Figure 3.18). 


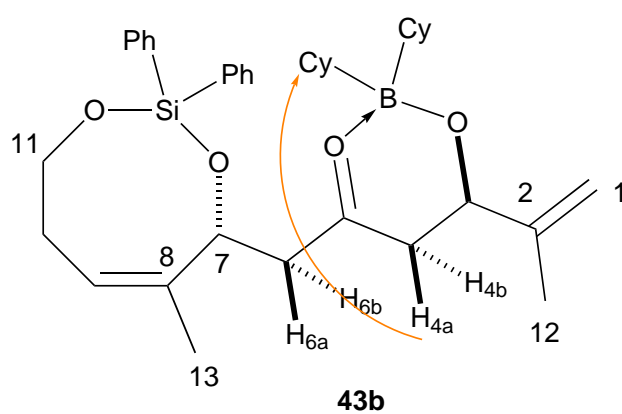

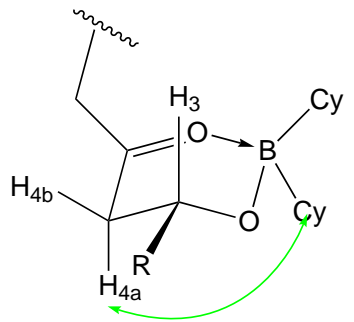

Boat conformer

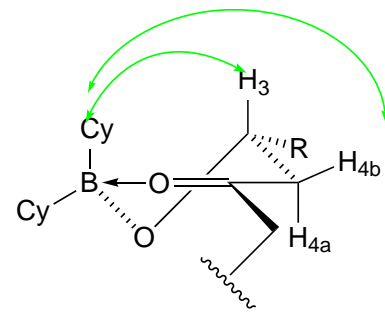

Chair conformer

Key:
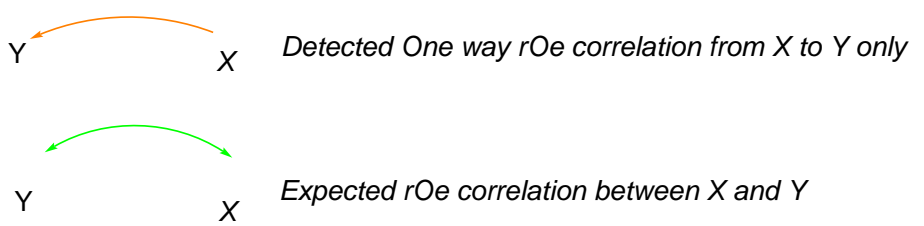

$R=\sqrt[5]{5}^{5}$

Figure 3.18: Key rOe Correlation of $\mathbf{4 3 b}$ and Expectated rOe Correlations in Different Conformers

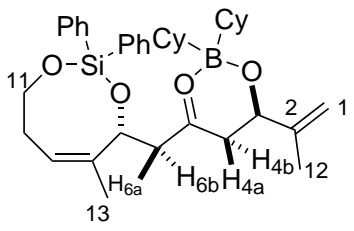

43b

\begin{tabular}{cll}
\hline Position & $\delta^{1} \mathrm{H}(\mathrm{ppm})$ & Key rOe correlation \\
\hline 3 & $4.83-4.80(\mathrm{~m}, 1 \mathrm{H})$ & \\
$4 \mathrm{a}$ & $2.86-2.78(\mathrm{~m}, 1 \mathrm{H})$ & Cyclohexyl proton(s) \\
$4 \mathrm{~b}$ & $2.60(\mathrm{dd}, J=15.9,2.9 \mathrm{~Hz}$, & \\
& $1 \mathrm{H})$ or $2.52(\mathrm{dd}, \mathrm{J}=15.1,3.2$ & \\
& $\mathrm{Hz}, 1 \mathrm{H})$ \\
$6 \mathrm{a}$ & $2.60(\mathrm{dd}, J=15.9,2.9 \mathrm{~Hz}$, & \\
& $1 \mathrm{H})$ or $2.52(\mathrm{dd}, \mathrm{J}=15.1,3.2$ & \\
& $\mathrm{Hz}, 1 \mathrm{H})$ \\
$6 \mathrm{~b}$ & $2.86-2.78(\mathrm{~m}, 1 \mathrm{H})$ \\
7 & $4.98(\mathrm{~d}, J=9.6 \mathrm{~Hz}, 1 \mathrm{H})$ \\
$10 \mathrm{a}$ & $2.86-2.78(\mathrm{~m}, 1 \mathrm{H})$ \\
\hline
\end{tabular}

Table 3.7: Key ${ }^{1} \mathrm{H}$ Spectral Data and rOe Correlation of $\mathbf{4 3 b}$ 
To understand more about the conformation of the boron-complexed aldol product and how it relates to the TSs leading to the dramatic difference in the stereoinduction between $\mathbf{2}$ and 3, NMR studies of the aldol reaction between acyclic silyl acetal $\mathbf{3}$ and methacrolein $\mathbf{2 7 a}$ using $\mathrm{Cy}_{2} \mathrm{BCl}$ and TMP were also carried out (Scheme 3.26). As shown in Scheme 3.19 (page 59), there was little selectivity for this aldol reaction, which gave a ca. 1:1 anti:syn ratio. Therefore, the resulting ${ }^{1} \mathrm{H}$ NMR spectrum of the reaction mixture contained two sets of proton signals in similar intensity corresponding to the two diastereomers. Fortunately, after running a COSY experiment with the reaction mixture and comparing the ${ }^{1} \mathrm{H}$ NMR spectrum with the starting ketone 3 , it was possible to reveal the positions of $\mathrm{H}_{3}, \mathrm{H}_{4}, \mathrm{H}_{6}, \mathrm{H}_{7}, \mathrm{H}_{13}$ and some other protons of boron-complexed products 44 (Table 3.8). Most of the protons and their corresponding ones in the other diastereomer overlap in the spectrum. Unless otherwise stated, the protons mentioned in the following content belong to both diastereomers. $\mathrm{H}_{6 a}$ and $\mathrm{H}_{6 b}$ were well separated in the spectrum and did not overlap with any other proton signals of 44; in comparison, $\mathrm{H}_{4 a}$ and $\mathrm{H}_{4 b}$ were more upfield than those of $\mathbf{4 2}$ and $\mathbf{4 3} \mathrm{b}$ and also well separated from each other but partially overlapping with $\mathrm{H}_{12}$. These signals can be still useful for the 1D ROESY conformational study of $\mathbf{4 4}$ since $\mathrm{H}_{12}$ is likely to be far away from the six-membered ring and not expected to correlate with the cyclohexyl substituent of the boron. Interestingly, $\mathrm{H}_{7}$ was embedded in the region of alkene protons at $4.71 \mathrm{ppm}$, while $\mathrm{H}_{3}$ was upfield at $4.51 \mathrm{ppm}$.

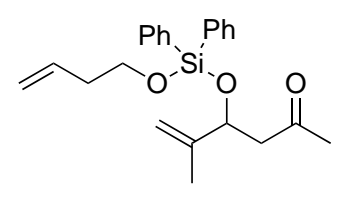

3

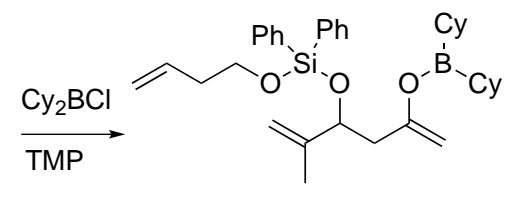

51

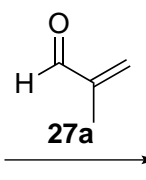

$27 a$

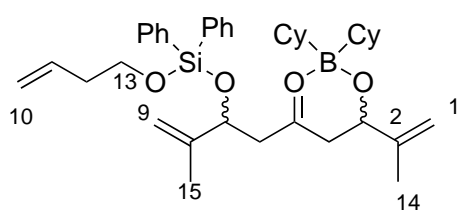

44

Scheme 3.26: Aldol Reaction of $\mathbf{3}$ with $\mathbf{2 7 a}$ in the NMR study

After revealing the positions of the protons in the spectrum, 1D ROESY was run at RT; the key ${ }^{1} \mathrm{H}$ NMR spectral data and rOe correlations are shown in Table 3.8 and Figure 3.19. Analogously to $43 \mathbf{b}$, there was no rOe correlation between $\mathrm{H}_{3}$ and the cyclohexyl group and a correlation between $\mathrm{H}_{4 a}$ (anti to $\mathrm{H}_{3}$ ) and the cyclohexyl group was present. This result again suggests a boat conformer is dominant in this system and this is demonstrated more convincingly than with the $1 \mathrm{D}$ ROESY result of $\mathbf{4 3} \mathbf{b}$ since both of $\mathrm{H}_{6}$ do not overlap with $\mathrm{H}_{4 a}$ or $\mathrm{H}_{4 b}$ in the ${ }^{1} \mathrm{H}$ spectrum of $\mathbf{4 4}$. For the boat conformer to completely overwhelm the chair one in the 1D ROESY experiment, there could be either one or more unfavorable interactions in the chair alone, which we did not see, or, more likely, an extra stabilizing force for the boat conformer. Goodman's hydrogen bond is the most plausible stabilizing force for the boat conformer here. If the boat conformers of $\mathbf{4 4}$ really structurally resemble the Goodman TS, $\mathrm{H}_{7}$ and $\mathrm{H}_{13}$ and certain other protons should also show up distinct rOe correlations with the cyclohexyl group as shown in Scheme 3.27 (page 87). Indeed, there appeared to be an rOe correlation between $\mathrm{H}_{7}$ and the cyclohexyl group (Figure 3.19 and Scheme 3.27) although no absolute conclusion can be made since the NMR peak for $\mathrm{H}_{7}$ was embedded in the alkene protons. $\mathrm{H}_{13}$ also showed 


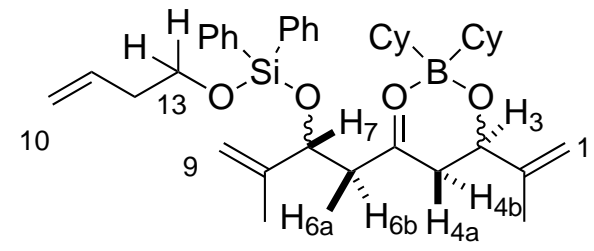

44

\begin{tabular}{|c|c|c|}
\hline Position & $\delta^{1} \mathrm{H}(\mathrm{ppm})$ & Key rOe correlation \\
\hline 3 & $4.51(\mathrm{dd}, J=6.8,3.8 \mathrm{~Hz}, 1 \mathrm{H})$ & \\
\hline $4 a$ & $\begin{array}{l}2.37 \text { (app. ddd, } J=14.1,7.1,1.8 \\
\mathrm{~Hz}, 1 \mathrm{H})\end{array}$ & Cyclohexyl protons \\
\hline $4 b$ & $2.29-2.21(\mathrm{~m}, 1 \mathrm{H})$ & \\
\hline $6 \mathrm{a}$ & $2.48(\mathrm{dd}, J=15.1,4.9 \mathrm{~Hz}, 1 \mathrm{H})$ & \\
\hline $6 \mathrm{~b}$ & $2.72(\mathrm{dd}, J=14.9,7.7 \mathrm{~Hz}, 1 \mathrm{H})$ & \\
\hline 7 & $4.72-4.69(\mathrm{~m}, 1 \mathrm{H})$ & Cyclohexyl protons \\
\hline $11 \mathrm{a}$ & $2.29-2.21(\mathrm{~m}, 1 \mathrm{H})$ & \\
\hline $11 b$ & $2.29-2.21(\mathrm{~m}, 1 \mathrm{H})$ & \\
\hline 13 & $3.70(\mathrm{t}, J=6.6 \mathrm{~Hz}, 2 \mathrm{H})$ & Cyclohexyl protons \\
\hline
\end{tabular}

Table 3.8: Key ${ }^{1} \mathrm{H}$ Spectral Data and rOe Correlations of $\mathbf{4 4}$

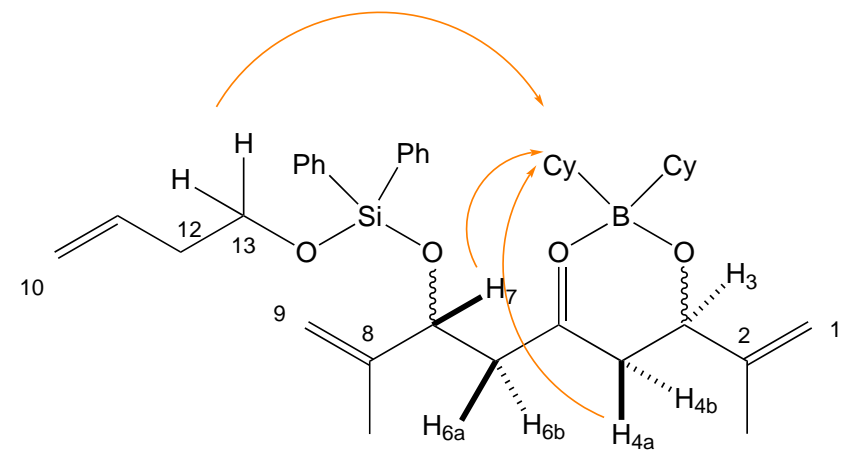

Key:

44

The ${ }^{1} \mathrm{H}$ spectrum of $\mathbf{4 4}$ contains two diastereomers relating to the relative stereochemstry of two oxymethine centers $\mathrm{C}_{3}-\mathrm{O}_{3}$ and $\mathrm{C}_{7}-\mathrm{O}_{7}$.

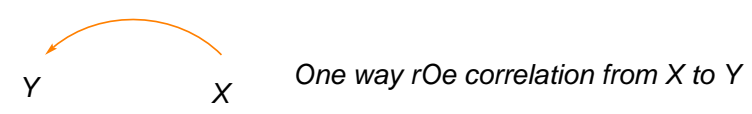

Figure 3.19: Key rOe Correlations of $\mathbf{4 4}$ 
an rOe correlation with the cyclohexyl group (Figure 3.19). These correlations are expected for Goodman's anti-boat conformer. These results suggest that Goodman's boat(s) might even be adopted by the aldol product 44. As stated previously, we also need to be clear about whether an alternative hydrogen bond occurs between the $\beta$-oxygen on $\mathrm{C}_{7}$ and one of the more acidic $\alpha$-protons $\mathrm{H}_{4}$.

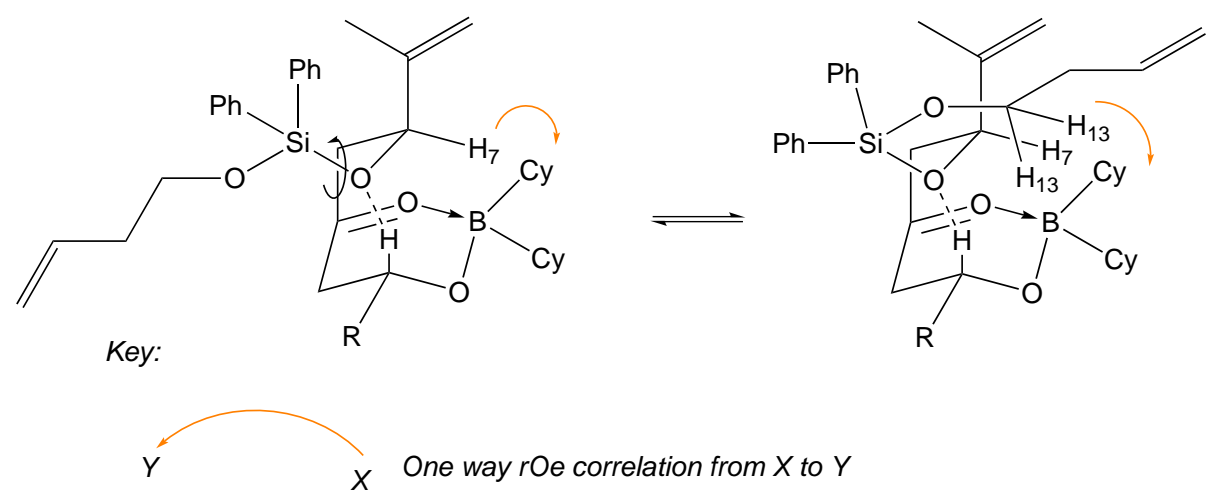

Scheme 3.27: A Goodman's anti-Boat Conformer Responsible for the rOe Correlations of $\mathrm{H}_{7}$ and $\mathrm{H}_{13}$ of Boron-Complexed Aldol Product 44

As shown in Scheme 3.28, where there is an $\mathrm{O}_{7^{-}}-\mathrm{H}_{4}$ hydrogen bond, 44 would become a bicyclic system $\mathbf{4 4 a}$, which has two six-membered rings, labelled $\mathbf{A}$ and $\mathbf{B}$. The validity of the hydrogen bond between $\mathrm{O}_{7}$ and $\mathrm{H}_{4}$ was judged based on the ability of the resulting stable conformation of $\mathbf{4 4 a}$ to satisfy all of the ROESY data. The relative stereochemistry mentioned in the following discussion and in Figures 3.20 and 3.21 is based on the relative stereochemistry of the two protected hydroxy groups at $\mathrm{C}_{3}$ and $\mathrm{C}_{7}$ of $\mathbf{4 4}$ (anti or syn). One can presume that the anti-chair-chair conformer (Figure 3.20) can form favorably because both ring $\mathbf{A}$ and ring $\mathbf{B}$ are in chair conformations with all of the bulky substituents in equatorial positions and the bicyclic moiety is a trans-decalin system. syn-44a is also able to form a chair-chair conformer with all of the bulky substituents in equatorial positions but the bicyclic moiety is the less stable cis-decalin system and it may convert to a syn-chair-boat conformer which also has all of the bulky substituents in equatorial positions (Figure 3.20). We realize that $\mathrm{H}_{7}$ and $\mathrm{H}_{13}$ in all of these stable conformers mentioned above are far away from the cyclohexyl group and the rOe correlations of $\mathbf{4 4}$ of these protons could therefore not be from these conformers. As shown in Figure 3.21, the rOe correlation from $\mathrm{H}_{7}$ to the cyclohexyl group can be achieved by the moderately sterically unfavorable syn-boat-chair and anti-boat-chair conformers of $44 \mathbf{a}$, however, $\mathrm{H}_{13}$ is still not close to the cyclohexyl group until 44a is twisted into the highly sterically unfavorable syn-boat-boat and anti-boat-boat conformers. These two boat-boat conformers are achieved at a high energy cost due to the eclipse between the diphenylsilyl group and the axial cyclohexyl substituent of the boron and so are unlikely to be responsible for the rOe correlations. Therefore, the rOe correlations of $\mathrm{H}_{7}$ and $\mathrm{H}_{13}$ are unlikely to be caused by a putative $\mathrm{O}_{7^{-}-\mathrm{H}_{4}}$ hydrogen bond. 


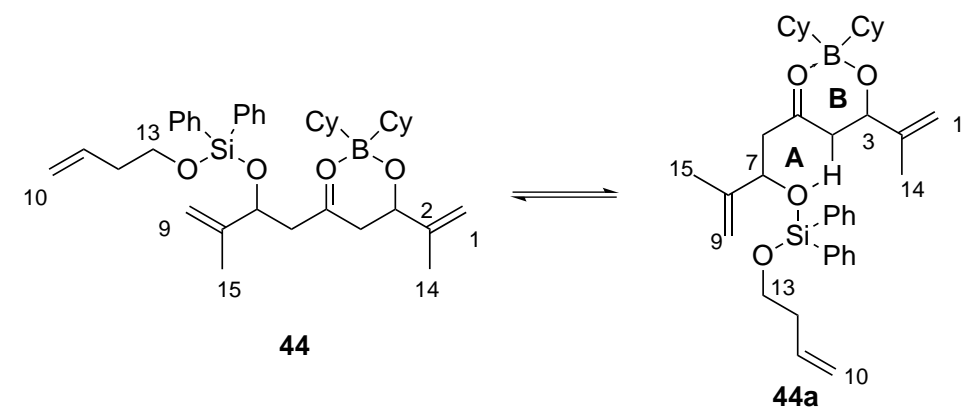

Scheme 3.28: An Unlikely Hydrogen bond between $\mathrm{O}_{7}$ and $\mathrm{H}_{4}$ in 44

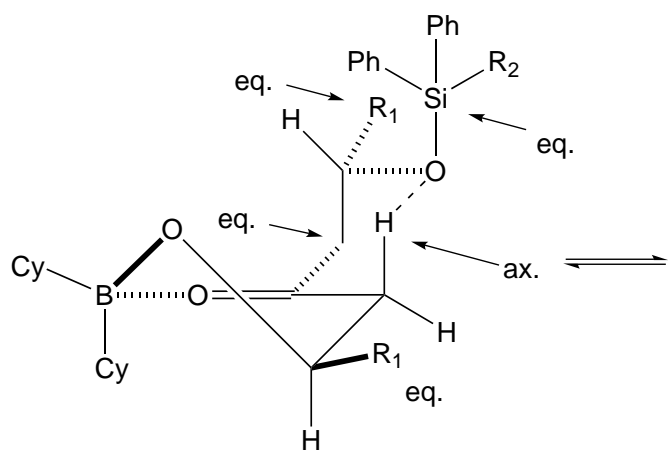

syn-chair-chair conformer

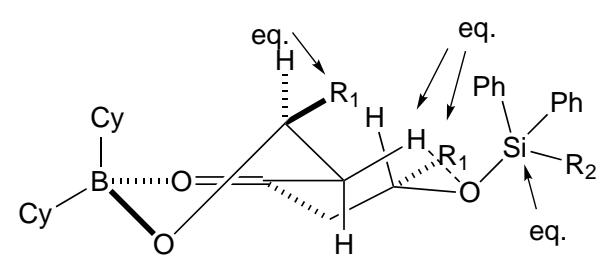

anti-chair-chair conformer

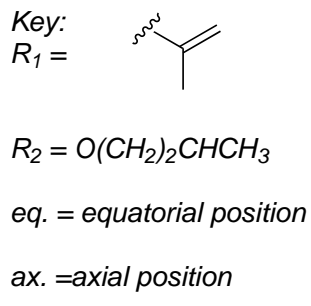

Figure 3.20: Expected Stable Conformers of 44a 


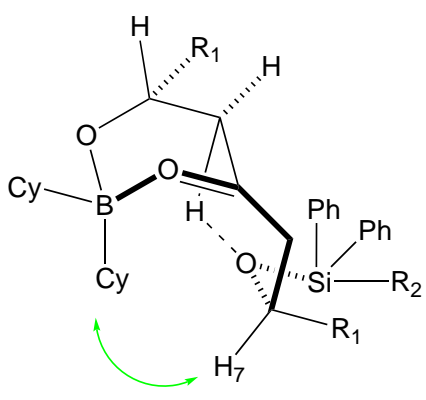

syn-boat-chair conformer

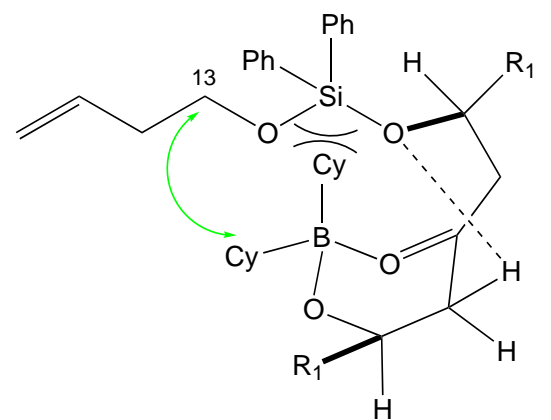

syn-boat-boat conformer

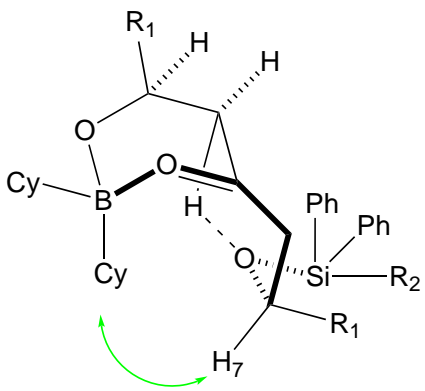

anti-boat-chair conformer

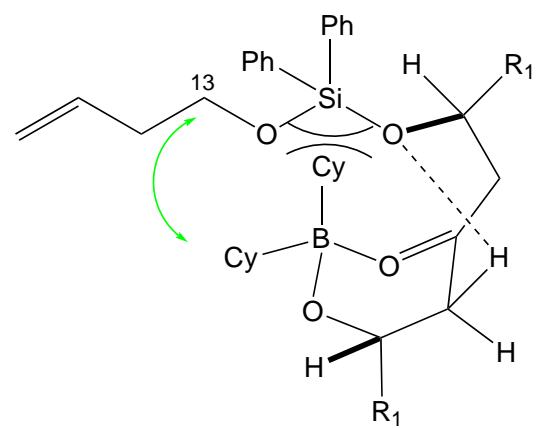

anti-boat-boat conformer

Key:

$R_{1}=s^{s^{5}}$

$\mathrm{R}_{2}=\mathrm{O}\left(\mathrm{CH}_{2}\right)_{2} \mathrm{CHCH}_{3}$

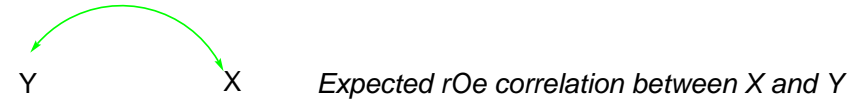

Figure 3.21: Unstable Conformers of $44 \mathbf{a}$ 
Bringing this all together, it is apparent that the boron-complexed aldol products of $\mathbf{2}$ and $\mathbf{3}$ (43b and 44, respectively) appear to retain a non-classical hydrogen bond that is analogous to that proposed by Goodman et al. in their model of the TS for the 1,5-anti aldol reaction, ${ }^{6}$ as the conformer that contains this interaction is the only reasonable conformer that can explain all the observed rOe correlations. Since the boat conformers of $\mathbf{4 3 b}$ and 44 were likely to be stabilized with a formal hydrogen bond, we expect that the hydrogen bond between the $\beta$-oxygen of the ketone and formyl hydrogen of the aldehyde would be even more stronger in the TS and so it can be tentatively deduced that Goodman's model is consistent with the results seen in this study.

\subsubsection{Proposed Mechanism for 1,5-anti Stereoinduction of 4a in Aldol Re- action}

After summarizing data from current and previous experiments, it appears that boat TSs modified from Goodman's model are consistent with the high stereoinduction of $\mathbf{2}$ in the aldol reaction. Apart from assuming that Goodman's proposed hydrogen bond exists in the TS, it is also assumed that the most stable conformer of the eight-membered ring of $\mathbf{2}$ in the TS of the aldol reaction is Crown $\mathbf{B}$ (Figure 3.7 on page 66). As shown in Figures 3.22 and 3.23, it is also noted that, apart from the two chiral centers at $\mathrm{C}_{3}$ and $\mathrm{C}_{7}$, a new chiral center is generated at $\mathrm{O}_{7}$ in the TS after the formation of Goodman's hydrogen bond; in other words, $\mathrm{O}_{7}$ is a prochiral center and its two lone pairs of electrons are diastereotopic to each other. This prochiral center plays an essential role in our proposed TS but was not focused on in Hoberg's and Goodman's models. Furthermore, based on the orientation of the $\mathrm{O}_{7^{-}}--\mathrm{H}_{3}$ hydrogen bond, boat-in and boat-out (hydrogen bond pointing into the ring and pointing out of the ring) conformers can be generated, in combination with the identity of the new chiral center $\mathrm{O}_{7}((R)$ - and $(S)$-). These are named as $(R)$-boat-in, $(R)$-boat-out, $(S)$-boat-in and $(S)$-boat-out and anti and syn prefixes are added in front of the labels to denote the 1,5-induction produced in each.

As shown in Figure 3.22, the two phenyl rings attached to the silicon of $\mathbf{2}$ can donate significant electron density to stabilize the empty $d$-orbital of the silicon such that the electronwithdrawing effect of the $d$-orbital largely decreases and the $\beta$-oxygen is now basic enough to form a proper Goodman's hydrogen bond in the TS. If the eight-membered ring adopts the Crown $\mathbf{B}$ conformer, it will have significant steric interference with the six-membered ring in all of the syn-boat conformers as illustrated in Figure 3.22. syn-(S)-boat-out and syn-(R)-boat-in conformers are especially unfavorable due to the strong steric repulsions between the phenyl rings and the equatorial $\mathrm{H}_{4}$ or the cyclohexyl group, respectively. The major interference in syn- $(R)$-boat-out is caused by the repulsions between the lone pair of electrons of $\mathrm{O}_{5}$ and the allylic methyl proton $\mathrm{H}_{13}$. Although the lone pair electrons of $\mathrm{O}_{7}$ may also repel $\mathrm{H}_{4}$, due to the relatively long hydrogen bond length, this repulsion may be very small. In the syn- $(S)$-boat-in conformer, $\mathrm{H}_{13}$ again mediates crucial steric interactions, causing a steric repulsion to $\mathrm{O}_{5}$ and 
cyclohexyl group. Additionally the lone pair of electrons of $\mathrm{O}_{7}$ is pointing into ring, thereby causing an unfavorable electron repulsion to the cyclohexyl group. Overall, the allylic methyl protons $\left(\mathrm{H}_{13}\right)$ are found to play an important role in destabilizing syn-boat conformers, such as syn- $(S)$-boat-in, syn- $(R)$-boat-out and syn- $(R)$-boat-in.

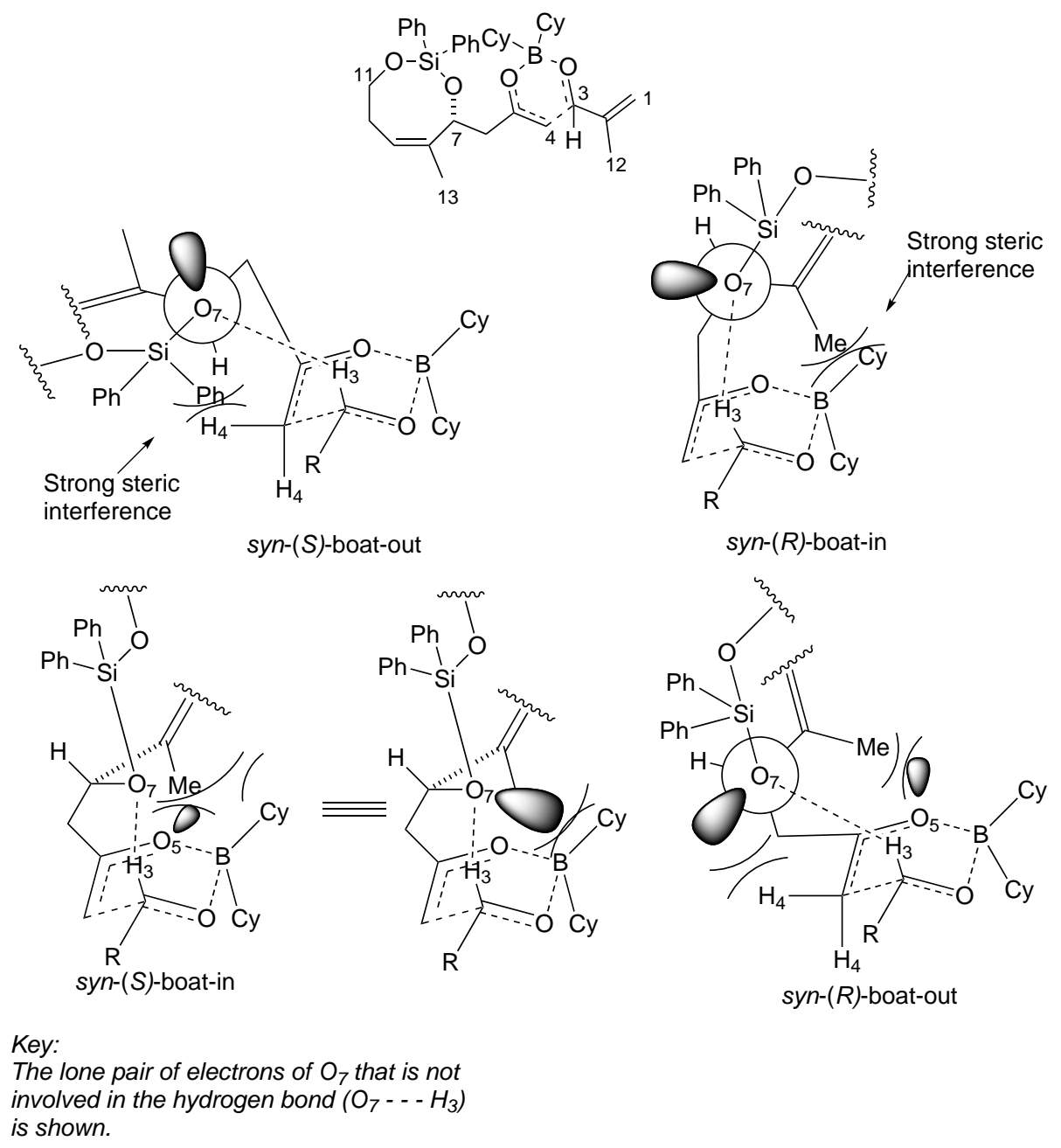

Figure 3.22: Modified Goodman's Syn-Boat Conformers for TS

In comparison, the steric factors that can interfere with the six-membered ring in anti-boats are less than those in the syn-boats (Figure 3.23). Furthermore, $\mathrm{H}_{13}$ is always lying outside of the six membered ring and not causing any unfavorable interference based on the four conformers in Figure 3.23. However, there are still two highly unfavorable conformers, anti- $(S)$-boat-in and anti- $(R)$-boat-out conformers. These are disfavored because of the strong steric repulsion between the phenyl ring and the cyclohexyl group and $\mathrm{H}_{4}$, respectively. In anti- $(R)$-boat-in, $\mathrm{O}_{7}$ may experience a moderate repulsion from the lone pair electron of $\mathrm{O}_{3}$ and the cyclohexyl substituent. In contrast to the other anti-boats, the only unfavorable steric interaction between $\mathrm{O}_{7}$ and equatorial $\mathrm{H}_{4}$ in the anti- $(S)$-boat-out may be very small because the long hydrogen bond length can increase the separation between $\mathrm{O}_{7}$ and $\mathrm{H}_{4}$. Therefore, the anti- $(S)$-boat-out 
conformer is a plausible TS for this aldol reaction as it has significantly less steric interference compared to the other anti-boats and syn-boats.
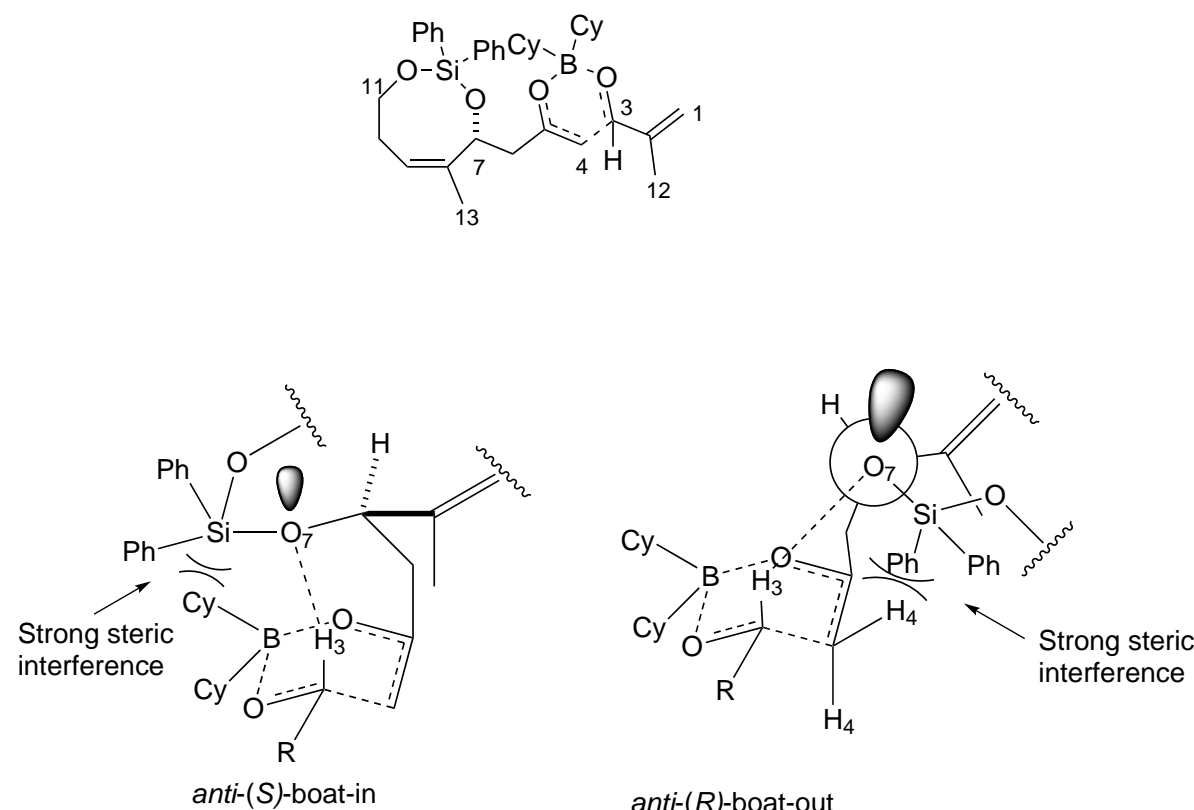

anti-(R)-boat-out
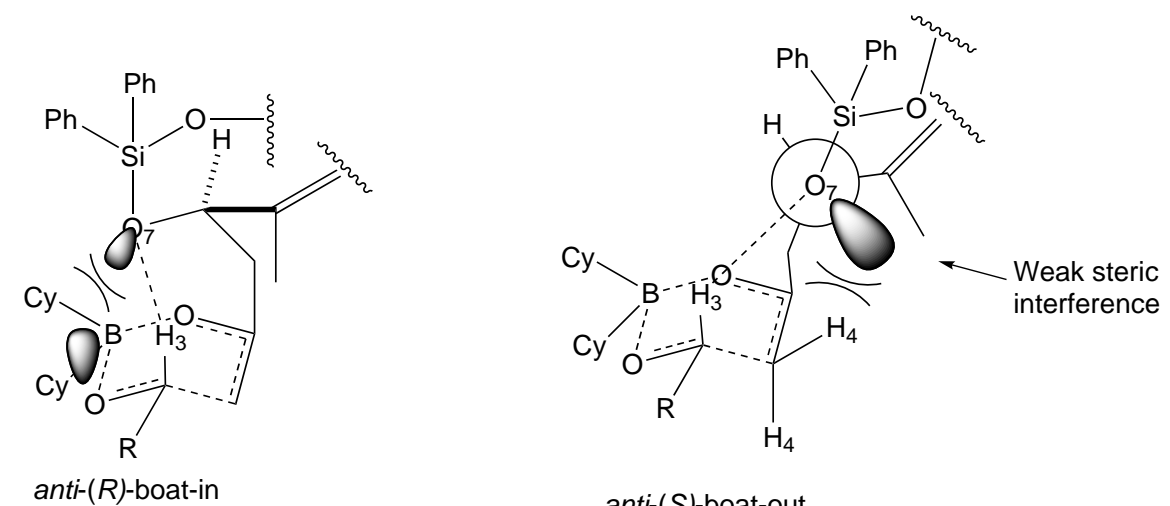

anti-(S)-boat-out

\begin{abstract}
Key:
The lone pair of electrons of $\mathrm{O}_{7}$ that is not involved in the hydrogen bond $\left(\mathrm{O}_{7}---\mathrm{H}_{3}\right)$
\end{abstract} is shown.

Figure 3.23: Modified Goodman's Anti-Boat Conformers for TS

This anti-( $S)$-boat-out model is able to explain the conflict between Goodman's calculation and Hoberg's results as described in Section 3.4.4 on page 62. The Goodman's hydrogen bond in the anti-(S)-boat-out conformer can orient the eight-membered diphenylsilyl acetal out of the six-membered ring, thus the steric interaction between the bulky diphenylsilyl acetal and the ring is minimized. The anti- $(S)$-boat-out conformer can also be adopted by diisopropylsilyl acetal protected alkyl methyl ketone (4 on page 26 ). However, as argued by Turner, ${ }^{2}$ because the lack of electron donating substituent attached to the silicon, the formyl hydrogen bond cannot be stabilized, thus the anti- $(S)$-boat-out conformer is not a predominant TS in the aldol 
reaction and a poor diastereoselectivity is resulted. In contrast to Goodman's TS, in which the terminal alkyl chain ( $\beta$-R group) played an important role in the stereoinduction (Figure 1.9 on page 28 ), the lone pairs electrons of the $\beta$-oxygen, the phenyl substituents, the allylic methyl group and the eight-membered ring of $\mathbf{2}$ are more important for its stereoinduction. The poor 1,5-anti stereoinduction of $\mathbf{3}$ can be due to the freely rotating diphenylsilyl acetal around the $\mathrm{Si}-\mathrm{O}$ bonds affecting the formation of a stable 1,5-anti $\mathrm{TS}$ and this intereference can be enhanced in the presence of a Goodman's hydrogen bond.

\subsubsection{Summary: Section 3.5}

In summary, the conformations of $\mathbf{2}$ and $\mathbf{3}$ were investigated with 1D NOESY experiments. The eight-membered diphenylsilyl acetal of $\mathbf{2}$ was found to adopt a Crown-B conformer (Figure 3.7 on page 66) which is also supported by the computational calculation. As expected, the diphenylsilyl acetal of acyclic 3 is freely rotating around both of the $\mathrm{Si}-\mathrm{O}$ bonds which could interfere with the approach of the aldehyde and boron reagent, leading to the lower yield compared to $\mathbf{2}$. The terminal boron enolates and aldol products of 2-hexanone, $\mathbf{2}$ and $\mathbf{3}$ were also investigated with modern NMR experiments. It was found that either DABCO or TMP can be used as the base for the boron-mediated aldol reaction and are beneficial for the ${ }^{1} \mathrm{H}$ NMR investigation by not blocking the 2.0-3.0 ppm region in the spectrum. There was no diborylated enolate detected in the ${ }^{1} \mathrm{H}$ NMR spectra of the three boron enolates, therefore the modified Abiko's mechanism for the 1,5-anti-stereoinduction of $\mathbf{2}$ was ruled out. Strong upfield-shifting effects caused by the anisotropic effect generally observed in the $\pi$-stacking are not apparent for $\mathrm{H}_{1 a}$ and $\mathrm{H}_{1 b}$ of the boron enolate, 48 , and the boat conformation of the boron-complexed aldol product, $43 \mathrm{~b}$, was deduced based on the rOe correlation data, both of which do not match Hoberg's $\pi$-stacking model. Therefore, Hoberg's model was also ruled out. The boat conformation of $\mathbf{4 4}$ was also found with the 1D ROESY experiment. Furthermore, the rOe correlations between $\mathrm{H}_{7}$ and $\mathrm{H}_{13}$ and the protons of the cyclohexyl group suggest that $\mathbf{4 4}$ exist in conformations consistent with Goodman's models. Therefore, the boron-mediated aldol reactions of $\mathbf{2}$ and $\mathbf{3}$ are likely to go through TSs similar to those of Goodman's model. A mechanism based on a modified Goodman's TS is proposed to explain the 1,5-anti-stereoinduction of $\mathbf{2}$. 


\subsection{Model Studies of $\mathrm{C}_{11}-\mathrm{C}_{12}$ Bond Connection in Syntheses of Peloruside A Analogues}

A series of boron-mediated aldol reactions of $\mathbf{2}$ with various aldehydes (Figure 3.24) have been conducted as model studies for connecting the $\mathrm{C}_{11}-\mathrm{C}_{12}$ bond of peloruside A (Table 3.9) as part of this work. However, cyclic $\mathbf{2}$ was not able to couple with aldehydes that are bulkier than methacrolein and similar results were found with acyclic 3 (Entries 1-8). Looking back at these unsuccessful results and comparing them with Turner's results, ${ }^{2}$ the quality of $\mathrm{Cy}_{2} \mathrm{BCl}$ was again suspected. This might account for the majority of the failed aldol reactions in our and Turner's studies. Increasing equivalents of $\mathrm{Cy}_{2} \mathrm{BCl}, \mathrm{Et}_{3} \mathrm{~N}$ and aldehyde were tried. For example, the aldol reaction of $\mathbf{2}$ with 6.0 eq. $\mathbf{2 7 f}$ (Figure 3.24) using 6.0 eq. $\mathrm{Cy}_{2} \mathrm{BCl}, 6.8$ eq. $\mathrm{Et}_{3} \mathrm{~N}$ provided a significant amount of the aldol product, as indicated by TLC and from crude ${ }^{1} \mathrm{H}$ NMR spectrum (Entry 9). However, due to the excess amounts of reagents in the reaction mixture, the product purification with flash chromatography was unsuccessful. In addition to the variable quality of boron reagent, the steric bulk of the ketones and the aldehydes could also be responsible for the failed aldol reactions since boron-mediated aldol reactions of $\mathbf{3}$ with the less bulky aldehyde, methacrolein (27a), using different equivalents of $\mathrm{Cy}_{2} \mathrm{BCl} / \mathrm{Et}_{3} \mathrm{~N}$ afforded a similar yield (26\%) (Entries 10 and 11). However, neither boron- nor lithium-mediated aldol reaction of $\mathbf{3}$ with $\mathbf{2 7 d}$ (Entries 12 and 13) was successful. As described in Section 3.4.3 (page 59 ), a titanium-mediated aldol reaction of acyclic $\mathbf{3}$ with $\mathbf{2 7} \mathbf{d}$ was achieved with a quantitative yield. Therefore, it was deemed worth while attempting the aldol reaction of cyclic ( $r a c)-2$ with a bulky aldehyde using $\mathrm{TiCl}_{4}$ as the mediating agent. Surprisingly, ( $\left.r a c\right)-\mathbf{2}$ entirely decomposed in the presence of $\mathrm{TiCl}_{4}$ and the crude ${ }^{1} \mathrm{H}$ NMR looked sufficiently complicated that it was assumed more than one by-product had formed (Entry 14). A similar observation was described by Turner; the $\mathrm{TiCl}_{4}$ mediated aldol reaction of $(S)$-6a did not provide any aldol product and the corresponding crude ${ }^{1} \mathrm{H}$ NMR was also very complicated. ${ }^{2}$ The dramatically different reactivity between $\mathbf{3}$ and $\mathbf{2}$ to $\mathrm{TiCl}_{4}$ might be due to the flexiblility of $\mathbf{3}$ in solution preventing the titanium inserting into the $\mathrm{Si}-\mathrm{O}$ bond.

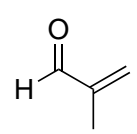

$27 a$

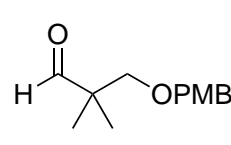

$27 e$<smiles>O=Cc1cccc([N+](=O)[O-])c1</smiles>

$27 f$

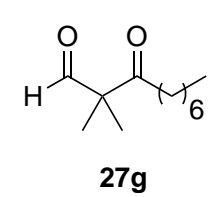

$27 \mathrm{~g}$

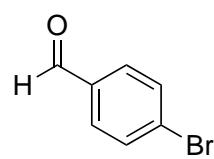

27d

Figure 3.24: Aldehydes Described in this Section

In conclusion, although 2 can direct high 1,5-anti stereoinduction in aldol reactions, its intrinsic steric bulk limits their reactivities towards sterically hindered or conjugated aldehydes. Increasing equivalents of $\mathrm{Cy}_{2} \mathrm{BCl}$ and aldehyde seemed to improve the yield, presumably due to the increase of "intact" $\mathrm{Cy}_{2} \mathrm{BCl}$ in the reaction mixture. $\mathrm{TiCl}_{4}$ is a stronger mediating agent than $\mathrm{Cy}_{2} \mathrm{BCl}$ and LDA and has been successfully used to couple ( $\left.r a c\right)-\mathbf{3}$ and $\mathbf{2 7} \mathbf{d}$; however, it was not compatible with ( $r a c)-2$, leading to a degradation. 


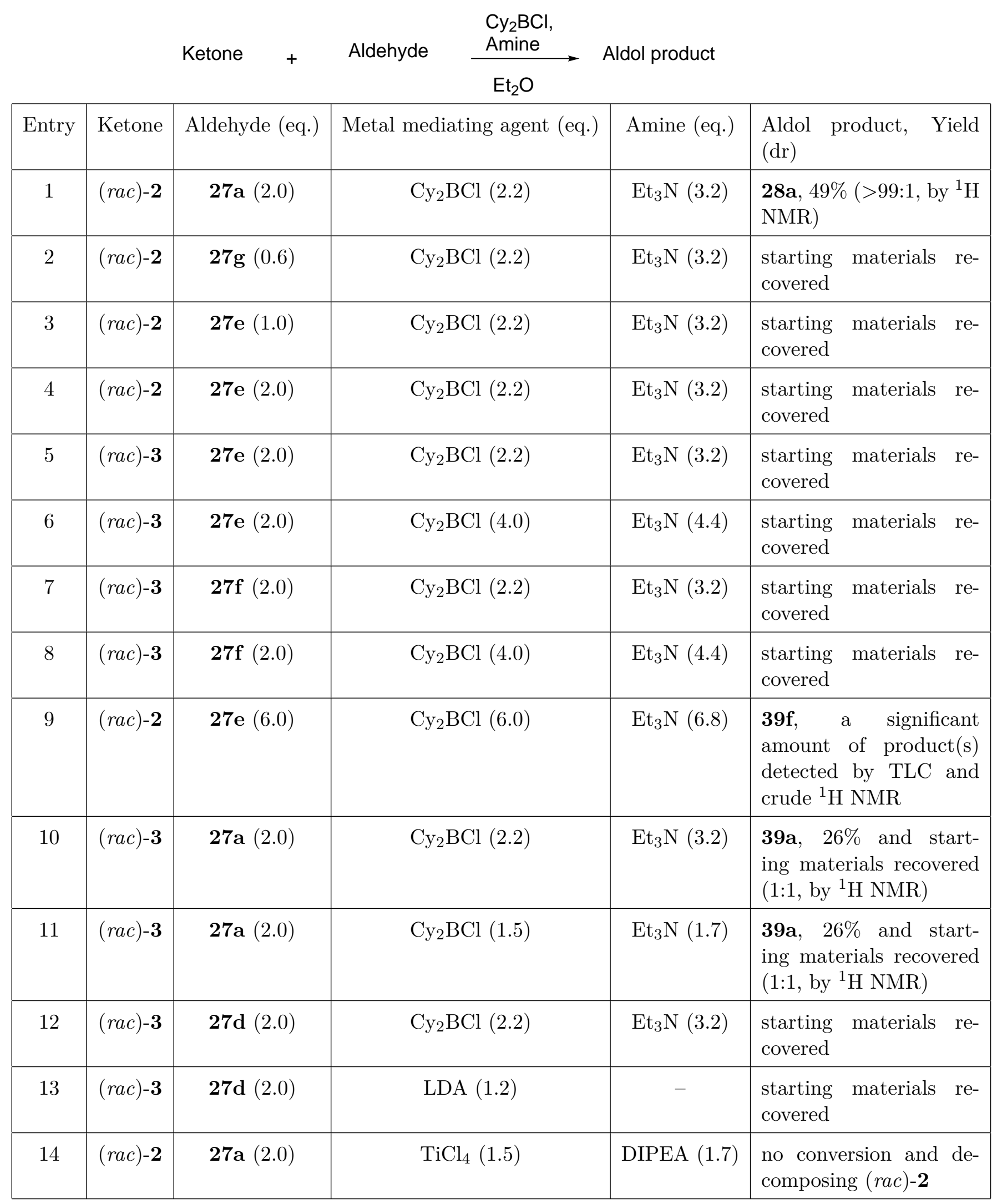

Table 3.9: Aldol Reactions of $\mathbf{2}$ and $\mathbf{3}$ with Various Aldehydes Investigated in the Model Studies 


\section{Chapter 4}

\section{Future Work}

\subsection{Future Work for Synthesis of 2}

Future studies will include improvements to the RCMs leading to $\mathbf{2}$ and $\mathbf{6 a}$. One method is a relay ring closing metathesis (RRCM) which has been successfully used in synthesizing mediumsized rings in many natural product syntheses, including a recent total synthesis of peloruside A by Hoye's group. ${ }^{64}$ The RRCM of silyl-tethered triene 52a provided a great improvement in yield by introduction of the teminal alkene "tail" (Reaction (1) in Scheme 4.1). The RRCM proccess involves cross-metathesis of the catalyst with the terminal alkene "tail" followed by a favorable RCM to form 3-methyl-1-cyclopentene and 1,1-disubstituted ruthenium carbene 52b. This undergoes the key RCM with the monosubstituted terminal alkene. Furthermore, volatile 3-methyl-1-cyclopentene is generated in this process which leads to a favorable entropic change for the reaction. Following this pathway, 52c and 52d could be potential candidates for a future RRCM route to desired $\mathbf{2}$ and $\mathbf{6 a}$ in high yield (Reactions (2) and (3), Scheme 4.1). Instead of performing RRCM with the terminal alkene "tail", using a conformationally restricted protecting group would bring the two alkenes closer and allow for a more effective RCM, such as 52e in Reaction (1), Scheme 4.2. Using an alternative protecting group for the pre-metathesis substrate, such as $\mathbf{5 2 f}$ in Reaction (2), Scheme 4.2, to form a larger sized ring would avoid the unfavorable medium-sized ring barrier and lead to a better yield. Since one of the major difficulties for the RCM leading to $\mathbf{2}$ and $\mathbf{6 a}$ is the unfavorable 1,1-disubstituted alkene, Rainier RCM could be an alternative strategy (Reaction (1) in Scheme 4.3). ${ }^{65}$ The benefit of Rainier RCM is the use of excess non-expensive $\mathrm{TiCl}_{4}$ and the generation of titanium oxide as a by-product in an irreversible process. Therefore, $\mathbf{5 2} \mathbf{g}$, a modified substrate, can be used to synthesize $\mathbf{2}$ and $\mathbf{6 a} .{ }^{65}$ A similar RCM to Rainier metathesis had been used by Takeda et al. ${ }^{66}$ starting with a thioacetal protected carbonyl group and 1,1-disubstituted alkene, affording a good yield (ca. 70\%). Therefore, substrate $\mathbf{5 2 h}$ may also be a good candidate for the RCM (Reaction (2), Scheme 4.3). 


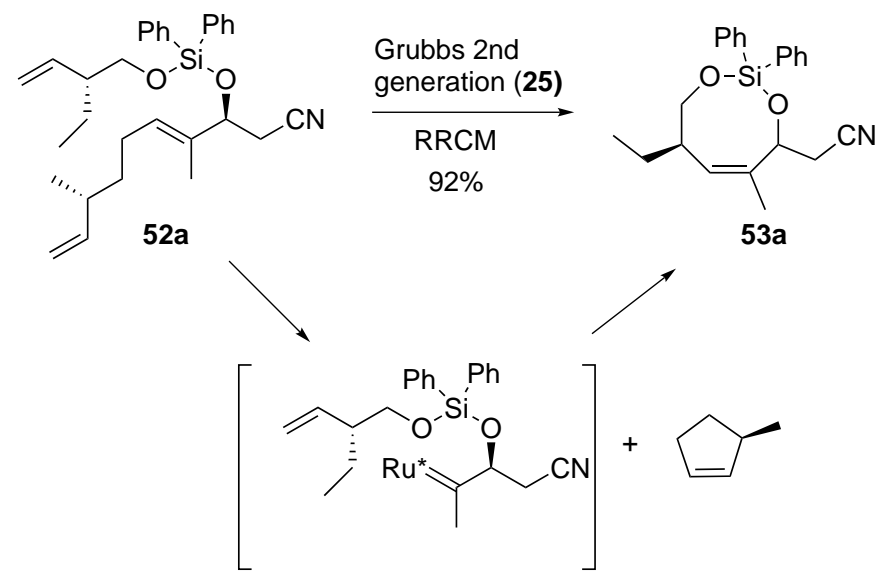

(1)

$R u^{*}=$ Ruthenium complex

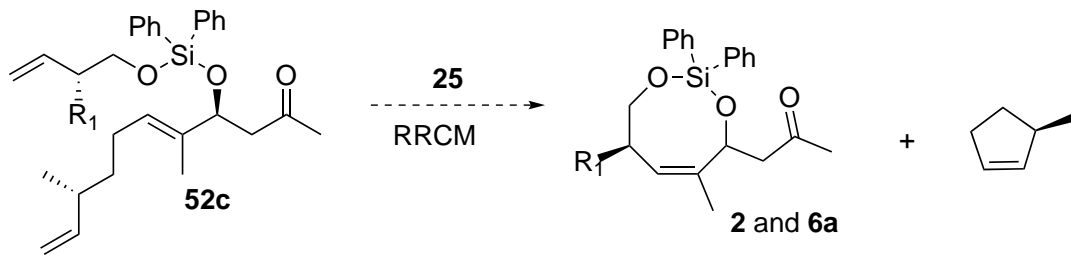

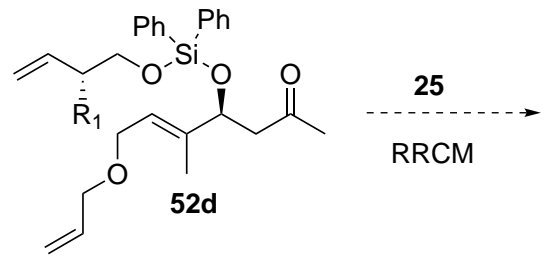<smiles>[R7]C1C=C([18CH])C(CC(C)=O)O[Si](c2ccccc2)(c2ccccc2)OC1</smiles>

$\mathrm{R}_{1}=\mathrm{H}$ or $\mathrm{CH}_{2} \mathrm{CH}_{3}$

Scheme 4.1: Hoye's RRCM ${ }^{64}$ (Reaction (1)) and Proposed RRCM (Reaction (2) and (3))<smiles>[R6]C1C=C(C)C([R6])Oc2ccccc2OC1</smiles>

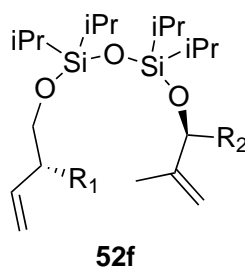
25

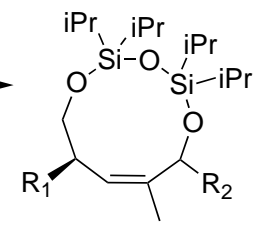

Scheme 4.2: Alternative Tethers for Metathesis Reactions 

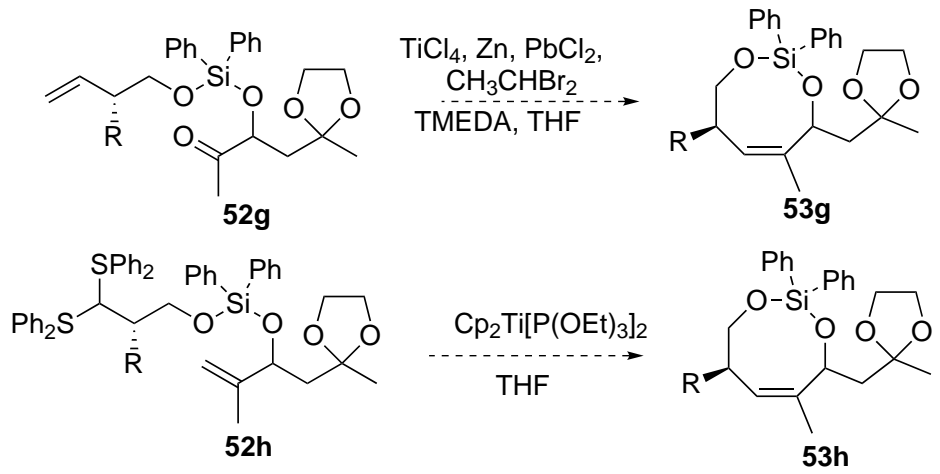

$\mathrm{R}=\mathrm{H}$ or $\mathrm{CH}_{2} \mathrm{CH}_{3}$

Scheme 4.3: Proposed Rainier and Takeda RCM in the Syntheses of $4 \mathrm{a}$ and $4 \mathrm{~b}^{65,66}$

\subsection{Future Work for Investigating the Underlying Stereoinduc- ing Mechanism of 2}

As described in Section 3.6, Hoberg's $\pi$-stacking and Abiko's doubly borylated models for explaining the 1,5-anti stereoinduction of $\mathbf{2}$ have been ruled out and boat-like conformations in the boron-complexed aldol products (43b) were deduced. However, there is still no direct evidence to support the aldol reaction of $\mathbf{2}$ going through a Goodman's 1,5-anti TS. Therefore, future study will include the aldol reaction of $\mathbf{5 4}$ with methacrolein (Reaction (1), Scheme 4.4). Since the Goodman's hydrogen bond is the essential factor for the stereoinduction, substituting the $\beta$-oxygen with a $\mathrm{CH}_{2}$ should largely diminish the stereoselectivity of the reaction if a Goodman's TS is formed, providing a more direct evidence. However, the lack of a $\beta$-oxygen means Kishi's NMR databse can not be applied to the determination of the relative stereochemistry of the aldol product, making this challenging. Alternatively, the validity of Goodman's model can be indirectly proven using different metal mediating agents (Reaction (2), Scheme 4.4). ${ }^{67-69}$ Because the small size of boron in the six-membered TS is essential for the formation of a Goodman's hydrogen bond, the aldol reaction of $\mathbf{2}$ mediated by another metal larger than boron is not expected to provide a highly stereoselective 1,5-anti-aldol product. The aldol reaction of 26a with methacrolein is also worth trying since the allylic methyl group was proposed to play a role in the stereoinduction of $\mathbf{2}$ in our model (Reaction (3), Scheme 4.4).

It has been shown that the eight-membered ring of $\mathbf{2}$ is essential for the excellent stereochemical outcome. To understand whether different ring sizes of diphenylsilyl acetals can affect the stereoselectivity to different degrees, the boron-mediated aldol reactions of $\mathbf{5 5}$ can be attempted (Reaction (4), Scheme 4.4). Furthermore, the potential application of diphenylsilyl acetal in 1,5-anti aldol reactions will also be understood through these aldol reactions.

Although the boron-mediated aldol reactions of $\mathbf{2}$ with $\mathbf{2 7 f}$ provided a low yield (38\%) according to Turner's result, ${ }^{2}$ and $\mathrm{TiCl}_{4}$ was incompatible with $\mathbf{2}$, there are still a number of metal mediating agents available for aldol coupling, such as stannous triflate ${ }^{67}$ (Reaction (5), 
Scheme 4.4). ${ }^{67}$ Tin has a larger size than titanium, thus the corresponding six-membered TS is expected to be much less compact, which might afford a higher yield.

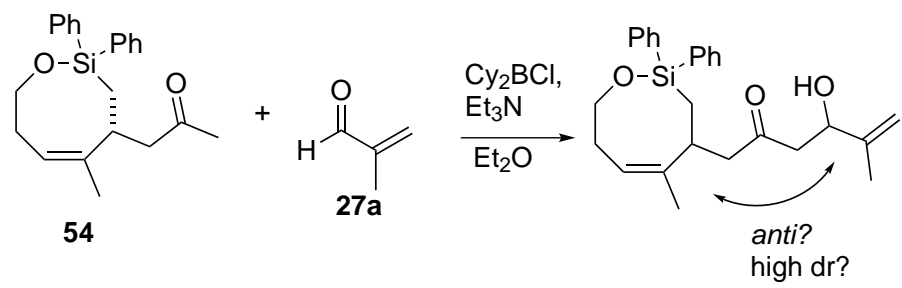<smiles>CC(=O)C[C@H]1O[Si](c2ccccc2)(c2ccccc2)OCCC=C1C</smiles>

2<smiles>C=C([18O])C=O</smiles>

27a<smiles>C=C([Te])C(=O)[OH2+]</smiles>

26a

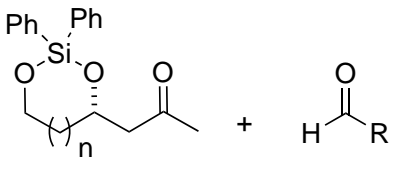

55 $\mathrm{n}=0,1,2 \ldots$<smiles>CC(=O)CC1O[Si](c2ccccc2)(c2ccccc2)OCCC=C1C</smiles><smiles>CCC(C)(C)C=O</smiles><smiles>C=C(C)C(O)CC(=O)CC1O[Si](c2ccccc2)(c2ccccc2)OCC(OCC)C=C1C</smiles>
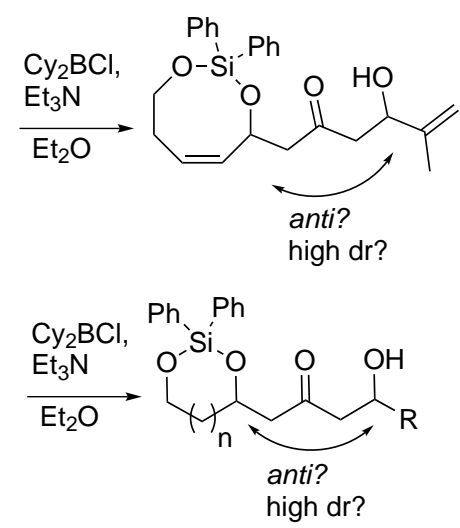

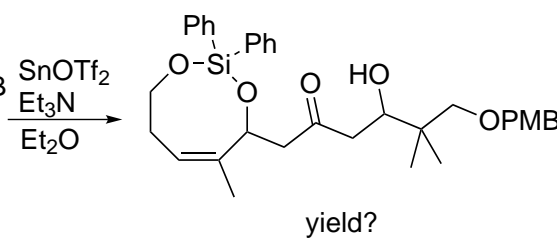

"M" = LHMDS, KHMDS, SnOTf/Et ${ }_{3} \mathrm{~N}, \mathrm{Me}_{2} \mathrm{AICl} / \mathrm{Et}_{3} \mathrm{~N}$ or $\mathrm{AIMe}_{3}$

Scheme 4.4: Proposed Aldol Reactions for Future Work 


\section{Chapter 5}

\section{Experimental Procedures}

\subsection{General}

Unless otherwise stated, the following conditions apply. All reagents were of commercial quality and distilled prior to use if necessary. The solvents were dried using standard procedures. All of the reactions were carried out under argon and standard syringe techniques were applied for transferring reagents to the reaction flask. Sodium hydride $(\mathrm{NaH})$ was obtained as a $60 \%$ suspension in mineral oil and was washed three times with distilled dry hexane before use. Reaction progress was monitored with aluminium backed or plastic backed TLC plates pre-coated with silica UV254 and visualized by $254 \mathrm{~nm}$ UV radiation and/or anisaldehyde dip or potassium permanganate dip. Silica gel 60 (220-240 mesh) was used in flash chromatography with the solvent system indicated in the method. All of the solvents used in flash chromatography were generally HPLC grade although normal grade hexane was occasionally used. ${ }^{1} \mathrm{H}$ and ${ }^{13} \mathrm{C}$ NMR spectra were recorded on either a Varian Unity Inova $300\left(300 \mathrm{MHz}\right.$ for ${ }^{1} \mathrm{H}$ and $75 \mathrm{MHz}$ for $\left.{ }^{13} \mathrm{C}\right)$, a Varian Unity Inova $500\left(500 \mathrm{MHz}\right.$ for ${ }^{1} \mathrm{H}$ and $125 \mathrm{MHz}$ for $\left.{ }^{13} \mathrm{C}\right)$, or a Varian DirectDrive $600\left(600 \mathrm{MHz}\right.$ for ${ }^{1} \mathrm{H}$ and $150 \mathrm{MHz}$ for $\left.{ }^{13} \mathrm{C}\right)$ spectrometer and referenced to solvent peaks $\left({ }^{1} \mathrm{H}\right.$ : residual $\mathrm{CHCl}_{3}$ or $\mathrm{CHD}_{2} \mathrm{OD},{ }^{13} \mathrm{C}: \mathrm{CDCl}_{3}$ or $\left.\mathrm{CD}_{3} \mathrm{OD}\right)$. Infrared spectra were obtained on a Bruker Tensor 27 FTIR spectrometer. Accurate masses were recorded with a Waters Q-TOF Premier $^{T M}$ Tandem Mass Spectrometer. The structure of each compound is presented with its method of preparation.

\subsection{Experiments}

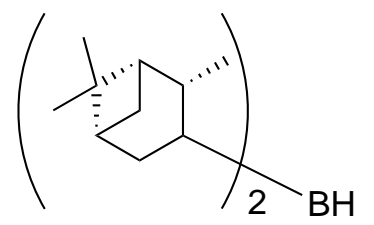

(+)-Diisopinocampheylborane: (-)- $\alpha$-Pinene (15.9 mL, $100 \mathrm{mmol})$ was added to THF $(15 \mathrm{~mL})$ in a flame-dried round bottom flask, followed by addition of dimethylsulfide borane complex $(4.17 \mathrm{~mL}, 41.7 \mathrm{mmol})$. The mixture was stirred and placed in a $-18{ }^{\circ} \mathrm{C}$ freezer overnight. The white suspension was warmed to RT and solvent was removed with a syringe. The solid was washed twice with $\mathrm{Et}_{2} \mathrm{O}(3 \mathrm{x} 10 \mathrm{~mL})$, the solvent was removed under 
reduced pressure and returned to atmospheric pressure by flushing with argon. It was then used directly in the next step.

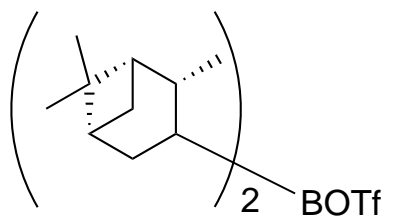

(+)-B-Trifluoromethanesulfonate diisopinocampheylborane $((+)-\mathbf{1 7 a})$ : Dried hexane $(9.2 \mathrm{~mL})$ was added to $(+)$ - $B$-diisopinocampheylborane $(11.9 \mathrm{~g}, 41.7 \mathrm{mmol})$ and cooled to $0{ }^{\circ} \mathrm{C}$. Triflic acid $(3.3$ $\mathrm{mL}$ ) was added dropwise and the mixture was warmed to RT until all of the solid reacted, giving two layers. The upper layer was a pale yellow color, the bottom one had orange semi-solid. The upper yellow layer was used directly in the following aldol reaction by using a syringe to measure the required volume $(60 \%$ conversion assumed based on Paterson's report, ${ }^{32}$ concentration $\left.=1.9 \mathrm{M}\right)$.

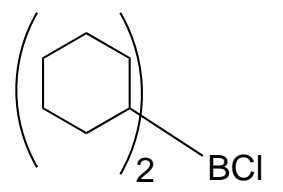

(+)-B-Chlorodiisopinocampheylborane (Reider's Method): To diglyme $(30 \mathrm{~mL})$ was added sodium borohydride $(38 \mathrm{mg}, 1.0 \mathrm{mmol})$. Cyclohexene $(0.41 \mathrm{~mL}, 330 \mathrm{mg}, 4.0 \mathrm{mmol})$ was added after the mixture was cooled to $-10{ }^{\circ} \mathrm{C}$. A solution of boron trichloride $(0.09 \mathrm{~mL}, 120 \mathrm{mg}, 1.0 \mathrm{mmol})$ was added slowly. The resulting mixture was stirred at $0{ }^{\circ} \mathrm{C}$ for $1 \mathrm{~h}$ and then returned to RT for $1 \mathrm{~h}$. The resulting solution was used directly in the boron-mediated aldol reactions of acetone with methacrolein. Comparing the yields of these aldol reactions with those of the literature, ${ }^{32}$ it was calculated that $\mathrm{Cy}_{2} \mathrm{BCl}$ was prepared in $17-25 \%$ yield.<smiles>C=C(C)[C@H](O)CC(C)=O</smiles>

(S)-4-Hydroxy-5-methylhex-5-en-2-one ((S)-18): $\mathrm{Et}_{3} \mathrm{~N}(3.4 \mathrm{~mL}, 2.4$ $\mathrm{g}, 24 \mathrm{mmol})$ was added to a solution of $(+)-(\mathrm{Ipc})_{2} \mathrm{BOTf}(5.5 \mathrm{~mL}, 1.9 \mathrm{M}$ solution in hexane) in $\mathrm{CH}_{2} \mathrm{Cl}_{2}(45 \mathrm{~mL})$ at $-78{ }^{\circ} \mathrm{C}$ followed, dropwise, by distilled acetone $(0.57 \mathrm{~mL}, 0.45 \mathrm{~g}, 7.7 \mathrm{mmol})$. The mixture was stirred for $1 \mathrm{~h} 45 \mathrm{~min}$, then distilled methacrolein $(1.3 \mathrm{~mL}, 15 \mathrm{mmol})$ was added dropwise and the reaction was run overnight (the dry ice in the $-78{ }^{\circ} \mathrm{C}$ cold bath evaporated off overnight). The reaction was quenched with pH 7 sodium phosphate buffer $(10 \mathrm{~mL})$. The solution was transferred to a separating funnel, and extracted with $\mathrm{CH}_{2} \mathrm{Cl}_{2}(2 \times 10 \mathrm{~mL})$, the organic phases were combined and the solvent was evaporated under reduced pressure. The residue was dissolved in $\mathrm{MeOH}(10 \mathrm{~mL})$, treated with $\mathrm{pH} 7$ sodium phosphate buffer solution $(10 \mathrm{~mL})$ and $30 \% \mathrm{H}_{2} \mathrm{O}_{2}$ solution $(7.5 \mathrm{~mL})$, and stirred for $2 \mathrm{~h}$. Water $(15 \mathrm{~mL})$ was added to the reaction mixture, which was transferred to a separating funnel. The aqueous layer was extracted with $\mathrm{CH}_{2} \mathrm{Cl}_{2}(3 \times 15 \mathrm{~mL})$. The organic fractions were combined, washed with sat. aq. brine $(15 \mathrm{~mL})$, dried with $\mathrm{MgSO}_{4}$, filtered and the solvent removed under reduced pressure. The pale yellow oil was purified by gradient flash chromatography (10:1 to 2:1 hexane:EtOAc) to yield $(S)-\mathbf{2}(0.44 \mathrm{~g}, 44 \%) .{ }^{1} \mathrm{H}$ NMR $(500 \mathrm{MHz}$, $\left.\mathrm{CDCl}_{3}\right) \delta 5.01(\mathrm{~s}, 1 \mathrm{H}), 4.86(\mathrm{~s}, 1 \mathrm{H}), 4.53-4.50(\mathrm{~m}, 1 \mathrm{H}), 2.91(\mathrm{~d}, J=2.5 \mathrm{~Hz}, 1 \mathrm{H}, \mathrm{OH}), 2.67(\mathrm{~d}$, $J=6.2 \mathrm{~Hz}, 2 \mathrm{H}), 2.20(\mathrm{~s}, 3 \mathrm{H}), 1.74(\mathrm{~s}, 3 \mathrm{H})$. Spectral data matched Turner's thesis. ${ }^{2}$ 


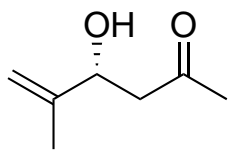

$(\boldsymbol{R})$-4-Hydroxy-5-methylhex-5-en-2-one $((\boldsymbol{R})-18): L$-proline $(120 \mathrm{mg}$, $1.0 \mathrm{mmol})$ was added to $50 \mathrm{~mL}$ of DMSO/acetone $(4: 1 \mathrm{v} / \mathrm{v})$ and the solution was stirred for $15 \mathrm{~min}$. The $L$-proline was only slightly dissolved. Methacrolein $(0.28 \mathrm{~mL}, 0.24 \mathrm{~g}, 3.4 \mathrm{mmol})$ was added and the reaction mixture was stirred overnight. The pale yellow mixture was transferred to the separating funnel and sat. aq. $\mathrm{NH}_{4} \mathrm{Cl}$ solution was added followed by extraction with ethyl acetate. The organic fraction was dried $\left(\mathrm{MgSO}_{4}\right)$, filtered and the solvent removed under reduced pressure. The pale yellow oil was passed through a pad of silica gel to remove the DMSO residue before purifying by fractional distillation under high vacuum $\left(58-60{ }^{\circ} \mathrm{C}, 2\right.$ mbar). A colorless oil was obtained $(0.35 \mathrm{~g}, 30 \%)$. ${ }^{1} \mathrm{H}$ NMR $(500 \mathrm{MHz}$, $\left.\mathrm{CDCl}_{3}\right) \delta 5.02(\mathrm{~s}, 1 \mathrm{H}), 4.87(\mathrm{~s}, 1 \mathrm{H}), 4.53-4.50(\mathrm{~m}, 1 \mathrm{H}), 2.91(\mathrm{~d}, J=2.5 \mathrm{~Hz}, 1 \mathrm{H}, \mathrm{OH}), 2.68(\mathrm{~d}$, $J=6.2 \mathrm{~Hz}, 2 \mathrm{H}), 2.21(\mathrm{~s}, 3 \mathrm{H}), 1.74(\mathrm{~s}, 3 \mathrm{H})$. Spectral data matched those reported in literature. ${ }^{4,43}$

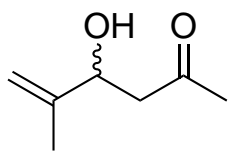

(rac)-4-Hydroxy-5-methylhex-5-en-2-one ((rac)-18): A 2 M solution of BuLi $(13.3 \mathrm{~mL}, 15.5 \mathrm{mmol})$ in cyclohexane was added to $\mathrm{iPr}_{2} \mathrm{NH}(2.20 \mathrm{~mL}$, $1.60 \mathrm{~g}, 15.5 \mathrm{mmol})$ in $\mathrm{THF}(50 \mathrm{~mL})$ at $-78{ }^{\circ} \mathrm{C}$. After $30 \mathrm{~min}$, acetone $(0.75$ $\mathrm{g}, 1.0 \mathrm{~mL}, 13.0 \mathrm{mmol}$ ) was added slowly and the reaction was run for $50 \mathrm{~min}$ at $-78{ }^{\circ} \mathrm{C}$, then freshly distilled methacrolein $(1.16 \mathrm{~mL}, 0.990 \mathrm{~g}, 14.2 \mathrm{mmol})$ was added dropwise. The reaction was stirred for $30 \mathrm{~min}$ and then quenched with sat. aq. $\mathrm{NH}_{4} \mathrm{Cl}$ solution. The mixture was warmed to $\mathrm{RT}$ and the aqueous layer was extracted with $\mathrm{CH}_{2} \mathrm{Cl}_{2}(3 \times 30 \mathrm{~mL})$. The organic fractions were combined and washed with sat. brine $(2 \times 20 \mathrm{~mL})$ and water $(3 \times 30 \mathrm{~mL})$, dried with $\mathrm{MgSO}_{4}$ and the solvent was removed with reduced pressure. The resulting yellow oil was distilled under high vacuum to give a pale yellow oil $(1.08 \mathrm{~g}, 65 \%) .{ }^{1} \mathrm{H}$ NMR (500 MHz, $\left.\mathrm{CDCl}_{3}\right) \delta 5.02(\mathrm{~s}, 1 \mathrm{H}), 4.87(\mathrm{~s}, 1 \mathrm{H}), 4.53-4.50(\mathrm{~m}, 1 \mathrm{H}), 2.91(\mathrm{~d}, J=2.5 \mathrm{~Hz}, 1 \mathrm{H}, \mathrm{OH}), 2.68(\mathrm{~d}, J$ $=6.1 \mathrm{~Hz}, 2 \mathrm{H}), 2.21(\mathrm{~s}, 3 \mathrm{H}), 1.74(\mathrm{~s}, 3 \mathrm{H})$. Spectral data matched Stocker's and Turner's theses. ${ }^{2,5}$

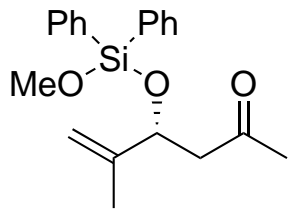

(R)-4-(Methoxydiphenylsilyloxy)-5-methyl-5-hexen-2-one $((R)$ 19a): Dichlorodiphenylsilane $(0.30 \mathrm{~g}, 1.2 \mathrm{mmol})$ was added to $\mathrm{CH}_{2} \mathrm{Cl}_{2}$ (5 $\mathrm{mL})$ at $0{ }^{\circ} \mathrm{C}$, followed by the addition of $\mathrm{Et}_{3} \mathrm{~N}(0.15 \mathrm{~mL}, 0.11 \mathrm{~g}, 1.2 \mathrm{mmol})$. After this, $(R)-\mathbf{1 8}(0.14 \mathrm{~g}, 1.1 \mathrm{mmol})$ was added and the mixture was warmed to reflux for $48 \mathrm{~h}$. The reaction mixture was cooled to $0{ }^{\circ} \mathrm{C}$ again, $\mathrm{Et}_{3} \mathrm{~N}(0.15 \mathrm{~mL}$, $0.11 \mathrm{~g}, 1.2 \mathrm{mmol})$ was added followed by the addition of methanol $(0.080 \mathrm{~mL}, 0.064 \mathrm{~g}, 2.0 \mathrm{mmol})$ and $\mathrm{CH}_{2} \mathrm{Cl}_{2}(5 \mathrm{~mL})$. The mixture was warmed to $\mathrm{RT}$ and stirred overnight. The reaction was quenched with sat. $\mathrm{NaHCO}_{3}(10 \mathrm{~mL})$. The organic phase was separated and the aqueous phase was extracted with $\mathrm{CH}_{2} \mathrm{Cl}_{2}(3 \times 10 \mathrm{~mL})$. The organic layers were combined and washed with sat. brine $(3 \times 10 \mathrm{~mL})$, dried with $\mathrm{MgSO}_{4}$, filtered and the solvent removed under reduced pressure. Flash chromatography (12:1 hexane:EtOAc) provided $(R)-\mathbf{1 9 a}(0.25 \mathrm{~g}, 30 \%)$ as a colorless oil. ${ }^{1} \mathrm{H}$ NMR $\left(500 \mathrm{MHz}, \mathrm{CDCl}_{3}\right) \delta$ 7.66-7.62 (m, 4H), 7.46-7.33 (m, 6H), $4.92(\mathrm{~s}, 1 \mathrm{H}), 4.80-4.78(\mathrm{~m}$, $2 \mathrm{H}), 3.57(\mathrm{~s}, 3 \mathrm{H}), 2.82(\mathrm{dd}, J=14.9,7.8 \mathrm{~Hz}, 1 \mathrm{H}), 2.57(\mathrm{dd}, J=14.9,4.8 \mathrm{~Hz}, 1 \mathrm{H}), 2.10(\mathrm{~s}, 3 \mathrm{H})$, $1.73(\mathrm{~s}, 3 \mathrm{H}) .{ }^{13} \mathrm{C} \mathrm{NMR}\left(125 \mathrm{MHz}, \mathrm{CDCl}_{3}\right) \delta 206.5,145.6,135.0,130.4,130.3,127.8,127.7,112.4$, 73.2, 51.2, 50.3, 30.9, 17.3. IR (neat) 3071, 3050, 2924, 2850, 1714, 1429, 1358, 1263, 1163, 1070, 1007, 900. HRMS (ESI) calcd. for $\mathrm{C}_{20} \mathrm{H}_{24} \mathrm{NaO}_{3} \mathrm{Si}\left(\mathrm{M}+\mathrm{Na}^{+}\right) 363.1392$, found 363.1394 . 


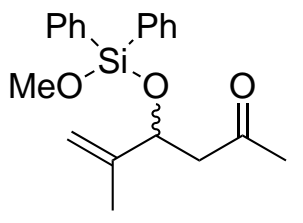

(rac)-4-(Methoxydiphenylsilyloxy)-5-methyl-5-hexen-2-one (( rac)19a): Method one: Following the same procedure as used for $(R)-\mathbf{1 9 a}$, (rac)-19a was prepared except (rac)-18 (0.14 g, $1.1 \mathrm{mmol})$. (rac)-19a was isolated as a clear oil $(0.25 \mathrm{~g}, 30 \%) .{ }^{1} \mathrm{H} \mathrm{NMR}\left(500 \mathrm{MHz}, \mathrm{CDCl}_{3}\right) \delta 7.66-7.62$ (complex m, 4H), 7.46-7.33 (complex m, 6H), $4.92(\mathrm{~s}, 1 \mathrm{H}), 4.80-4.78$ (m, 2H), 3.57 (s, 3H), 2.82 $(\mathrm{dd}, J=14.9,7.8 \mathrm{~Hz}, 1 \mathrm{H}), 2.57(\mathrm{dd}, J=14.9,4.8 \mathrm{~Hz}, 1 \mathrm{H}), 2.10$ (s, 3H), 1.73 (s, 3H). Spectral data matched $(R)$-19a.

Method two: Dichlorodiphenylsilane $(0.30 \mathrm{~g}, 1.2 \mathrm{mmol})$ was added to $\mathrm{CH}_{2} \mathrm{Cl}_{2}(5 \mathrm{~mL})$ at $0{ }^{\circ} \mathrm{C}$ followed by the addition of $\mathrm{Et}_{3} \mathrm{~N}(0.17 \mathrm{~mL}, 0.12 \mathrm{~g}, 1.2 \mathrm{mmol})$ and $\mathrm{AgNO}_{3}(0.34 \mathrm{~g}, 2.0 \mathrm{mmol})$. After this, (rac)-18 (0.14 g, $1.1 \mathrm{mmol})$ was added and the mixture was run at RT overnight. The reaction mixture was cooled to $0{ }^{\circ} \mathrm{C}$ again, $\mathrm{Et}_{3} \mathrm{~N}(0.17 \mathrm{~mL}, 0.12 \mathrm{~g}, 1.2 \mathrm{mmol})$ was added followed by the addition of methanol $(0.080 \mathrm{~mL}, 0.064 \mathrm{~g}, 2.0 \mathrm{mmol})$ and $\mathrm{CH}_{2} \mathrm{Cl}_{2}(5 \mathrm{~mL})$. The mixture was warmed to RT and stirred overnight. The reaction was quenched with sat. aq. $\mathrm{NaHCO}_{3}$ $(10 \mathrm{~mL})$. The organic phase was separated and the aqueous phase was extracted with $\mathrm{CH}_{2} \mathrm{Cl}_{2}$ $(3 \times 10 \mathrm{~mL})$. The organic layers were combined and washed with sat. brine $(3 \times 10 \mathrm{~mL})$, dried with $\mathrm{MgSO}_{4}$, filtered and the solvent removed with reduced pressure. Flash chromatography (12:1 hexane:EtOAc) provided ( rac)-19b. No ( rac)-19a was detected.

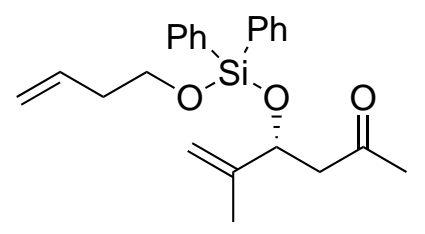

(R)-4-(But-3'-en-1'-oxydiphenylsilyloxy)-5-methyl-5-hexen2-one (( $R$ )-3): Dichlorodiphenylsilane $(0.30 \mathrm{~g}, 1.2 \mathrm{mmol})$ was added to $\mathrm{CH}_{2} \mathrm{Cl}_{2}(5 \mathrm{~mL})$ at $0{ }^{\circ} \mathrm{C}$ followed by $\mathrm{Et}_{3} \mathrm{~N}(0.17 \mathrm{~mL}, 0.12 \mathrm{~g}, 1.2$ mmol). After this, 3-buten-1-ol $(0.12 \mathrm{~mL}, 0.10 \mathrm{~g} 1.3 \mathrm{mmol})$ was added and the mixture was warmed to reflux for $48 \mathrm{~h}$. The reaction mixture was cooled to $0{ }^{\circ} \mathrm{C}$ again, $\mathrm{NEt}_{3}(0.17 \mathrm{~mL}, 0.12 \mathrm{~g}, 1.2 \mathrm{mmol})$ was added followed by the addition of $(R)-\mathbf{1 8}(0.14 \mathrm{~g}, 1.1 \mathrm{mmol})$ and $\mathrm{CH}_{2} \mathrm{Cl}_{2}(5 \mathrm{~mL})$. The mixture was warmed to RT and stirred overnight. The reaction was quenched with sat. aq. $\mathrm{NaHCO}_{3}(10 \mathrm{~mL})$. The organic phase was separated and the aqueous phase was extracted with $\mathrm{CH}_{2} \mathrm{Cl}_{2}(3 \times 10 \mathrm{~mL})$. The organic layers were combined and washed with sat. aq. brine $(3 \times 10 \mathrm{~mL})$, dried with $\mathrm{MgSO}_{4}$, filtered and the solvent removed under reduced pressure. Flash chromatography (10:1 hexane:EtOAc) provided diene $(R)-\mathbf{3}(0.25 \mathrm{~g}, 65 \%)$ as a colorless oil. ${ }^{1} \mathrm{H}$ NMR $\left(500 \mathrm{MHz}, \mathrm{CDCl}_{3}\right) \delta 7.65-7.62$ $(\mathrm{m}, 4 \mathrm{H}), 7.43-7.33(\mathrm{~m}, 6 \mathrm{H}), 5.82(\mathrm{ddt}, J=17.1,10.2,6.8 \mathrm{~Hz}, 1 \mathrm{H}), 5.09-5.05(\mathrm{~m}, 1 \mathrm{H}), 5.04-5.02$ $(\mathrm{m}, 1 \mathrm{H}), 4.90(\mathrm{~s}, 1 \mathrm{H}) 4.79-4.77(\mathrm{~m}, 2 \mathrm{H}), 3.77(\mathrm{t}, J=6.9 \mathrm{~Hz}, 2 \mathrm{H}), 2.80(\mathrm{dd}, J=14.9,7.7 \mathrm{~Hz}$, $1 \mathrm{H}), 2.55(\mathrm{dd}, J=14.9,5.0 \mathrm{~Hz}, 1 \mathrm{H}), 2.32$ (app. qt, $J=6.8,1.2 \mathrm{~Hz}, 2 \mathrm{H}), 2.07$ (s, 3H), 1.71 (s, $3 \mathrm{H})$. Spectral data matched those in the literature. ${ }^{4}$ 


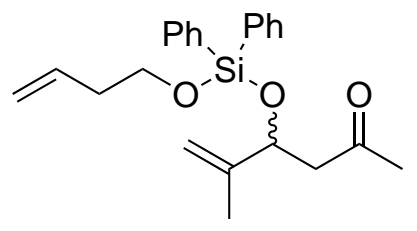

(rac)-4-(But-3'-en-1'-oxydiphenylsilyloxy)-5-methyl-5-hexen-

2-one $((\mathbf{r a c})-\mathbf{3})$ : Following the same procedure as $(R)-\mathbf{3}$, the synthesis of $(\mathrm{rac}) \mathbf{- 3}$ was prepared from $(\mathrm{rac}) \mathbf{- 1 8}(0.14 \mathrm{~g}, 1.1 \mathrm{mmol})$.

Flash chromatography (10:1 hexane:EtOAc) provided ( $r a c)-\mathbf{3}(0.25$ g, $65 \%)$ as a colorless oil. ${ }^{1} \mathrm{H}$ NMR $\left(500 \mathrm{MHz}, \mathrm{CDCl}_{3}\right) \delta 7.65-7.62$ $(\mathrm{m}, 4 \mathrm{H}), 7.43-7.33(\mathrm{~m}, 6 \mathrm{H}), 5.82(\mathrm{ddt}, J=17.1,10.2,6.8 \mathrm{~Hz}, 1 \mathrm{H}), 5.09-5.05(\mathrm{~m}, 1 \mathrm{H}), 5.04-5.02$ $(\mathrm{m}, 1 \mathrm{H}), 4.90(\mathrm{~s}, 1 \mathrm{H}), 4.79-4.77(\mathrm{~m}, 2 \mathrm{H}), 3.77(\mathrm{t}, J=6.9 \mathrm{~Hz}, 2 \mathrm{H}), 2.80(\mathrm{dd}, J=14.9,7.7 \mathrm{~Hz}$, $1 \mathrm{H}), 2.55(\mathrm{dd}, J=14.9,5.0 \mathrm{~Hz}, 1 \mathrm{H}), 2.32$ (app. qt, $J=6.8,1.2 \mathrm{~Hz}, 2 \mathrm{H}), 2.07$ (s, 3H), 1.71 (s, $3 \mathrm{H}) .{ }^{1} \mathrm{H}$ spectral data match those of $(R)-3$.<smiles>C=C(C)[C@H](CC(C)=O)O[Si](c1ccccc1)(c1ccccc1)c1ccccc1</smiles>

(rac)-4-( tert-Buyldiphenylsiloxy)-5-methyl-5-hexen-2-one (( rac)5): To a solution of $(R)-\mathbf{1 8}(0.13 \mathrm{~g}, 1.0 \mathrm{mmol})$ in DMF $(1.5 \mathrm{~mL})$ was added tert-Butyldiphenylsilyl chloride $(0.54 \mathrm{~g}, 2.0 \mathrm{mmol})$ and imidazole $(0.14 \mathrm{~g}$, $2.0 \mathrm{mmol}$ ). The reaction was run overnight before being quenched with sat. $\mathrm{NH}_{4} \mathrm{Cl}$ and then extracted with diethyl ether. The organic fractions were washed with brine and dried with $\mathrm{MgSO}_{4}$. The solvent was removed under reduce pressure and the crude oil was purified by flash chromatography (20:1 hexane:EtOAc) to afford a colorless oil $(0.20 \mathrm{~g}, 54 \%)$. ${ }^{1} \mathrm{H}$ NMR $\left(500 \mathrm{MHz}, \mathrm{CDCl}_{3}\right) \delta$ 7.68-7.64 (m, 4H), 7.46-7.35 (m, 6H), $4.79(\mathrm{~s}, 1 \mathrm{H}), 4.72(\mathrm{~s}, 1 \mathrm{H})$, $4.60(\mathrm{t}, J=6.4 \mathrm{~Hz}, 1 \mathrm{H}), 2.62(\mathrm{dd}, J=14.7,5.9 \mathrm{~Hz}, 1 \mathrm{H}), 2.53(\mathrm{dd}, J=14.7,6.4 \mathrm{~Hz}, 1 \mathrm{H}), 2.10$ $(\mathrm{s}, 3 \mathrm{H}), 1.68(\mathrm{~s}, 3 \mathrm{H}), 1.06(\mathrm{~s}, 9 \mathrm{H})$. Spectral data matched those reported in the literature. ${ }^{4}$

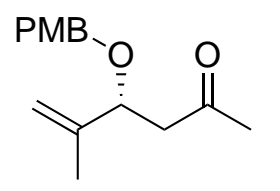

(R)-4-(4'-Methoxybenzyloxy)-5-methyl-hex-5-en-2-one $((R)-20)$ : To a solution of ( rac $) \mathbf{- 1 8}(32 \mathrm{mg}, 0.26 \mathrm{mmol})$ in $\mathrm{CH}_{2} \mathrm{Cl}_{2}(0.5 \mathrm{~mL})$ was added 4-methoxybenzyltrichloroacetimidate $(0.54 \mathrm{mmol})$ in $\mathrm{CH}_{2} \mathrm{Cl}_{2}(2 \mathrm{~mL})$. A catalytic amount of triflic acid or $( \pm)$-CSA was then added and the resulting mixture was stirred overnight at RT followed by quenching with sat. $\mathrm{NaHCO}_{3}(10 \mathrm{~mL})$ and then extracted with $\mathrm{CH}_{2} \mathrm{Cl}_{2}(3 \times 10 \mathrm{~mL})$. The organic fraction was dried with $\mathrm{MgSO}_{4}$ and the solvent was removed under reduced pressure. The resulting oil was dissolved in hexane and the white solid filtered off. The crude oil was purified with flash chromatography (5:1 hexane:EtOAc) provided (rac)-20 (44 mg, $60 \%$ ) as a colorless oil. ${ }^{1} \mathrm{H} \mathrm{NMR}\left(500 \mathrm{MHz}, \mathrm{CDCl}_{3}\right) \delta$ 7.23-7.22 (m, $2 \mathrm{H}), 6.88-6.86(\mathrm{~m}, 2 \mathrm{H}), 5.04(\mathrm{~s}, 1 \mathrm{H}), 4.99(\mathrm{~s}, 1 \mathrm{H}), 4.41(\mathrm{~d}, J=11.2 \mathrm{~Hz}, 1 \mathrm{H}), 4.26(\mathrm{dd}, J=9.0$, $3.9 \mathrm{~Hz}, 1 \mathrm{H}), 4.22(\mathrm{~d}, J=11.0 \mathrm{~Hz}, 1 \mathrm{H}), 3.80(\mathrm{~s}, 3 \mathrm{H}), 2.84(\mathrm{dd}, J=15.4,9.3 \mathrm{~Hz}, 1 \mathrm{H}), 2.45(\mathrm{dd}, J$ $=15.4,3.7 \mathrm{~Hz}, 1 \mathrm{H}), 2.15(\mathrm{~s}, 3 \mathrm{H}), 1.73(\mathrm{~s}, 3 \mathrm{H})$. Spectral data matched those reported by Turner. ${ }^{2}$

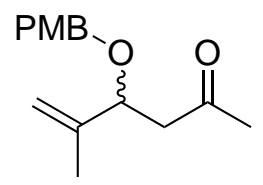

(rac)-4-(4'-Methoxybenzyloxy)-5-methyl-hex-5-en-2-one ((rac)-20): The same procedures as used for $(R)-\mathbf{2 0}$ were applied to the synthesis of $(\mathrm{rac})$ $\mathbf{2 0}$ with starting material ( $r a c)-\mathbf{1 8}(30 \mathrm{mg}, 0.26 \mathrm{mmol})$. This provided ( $\mathrm{rac}) \mathbf{- 2 0}$ in $60 \%$ (40 mg) yield. ${ }^{1} \mathrm{H} \mathrm{NMR}\left(500 \mathrm{MHz}, \mathrm{CDCl}_{3}\right) \delta 7.23-7.22(\mathrm{~m}, 2 \mathrm{H}), 6.88-$ $6.86(\mathrm{~m}, 2 \mathrm{H}), 5.04(\mathrm{~s}, 1 \mathrm{H}), 4.99(\mathrm{~s}, 1 \mathrm{H}), 4.41(\mathrm{~d}, J=11.2 \mathrm{~Hz}, 1 \mathrm{H}), 4.26(\mathrm{dd}, J=9.0,3.9 \mathrm{~Hz}$, $1 \mathrm{H}), 4.22(\mathrm{~d}, J=11.0 \mathrm{~Hz}, 1 \mathrm{H}), 3.80(\mathrm{~s}, 3 \mathrm{H}), 2.84(\mathrm{dd}, J=15.4,9.3 \mathrm{~Hz}, 1 \mathrm{H}), 2.45(\mathrm{dd}, J=$ 
15.4, 3.7 Hz, 1H), $2.15(\mathrm{~s}, 3 \mathrm{H}), 1.73(\mathrm{~s}, 3 \mathrm{H}) .{ }^{1} \mathrm{H}$ spectral data matched those reported for $(R)-\mathbf{2 0}$.

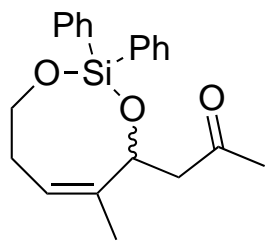

(rac)-2,2-Diphenyl-5-methyl-4-(2'-oxopropyl)-7,8-dihydro-4H-1,3dioxa-2-silocine (rac)-(2): To a solution of diene ( rac $)-\mathbf{3}(0.34 \mathrm{~g}, 0.90$ $\mathrm{mmol})$ in $\mathrm{CH}_{2} \mathrm{Cl}_{2}(100 \mathrm{~mL})$ was added Grubbs 2nd generation catalyst $\mathbf{2 5}$ $(0.090 \mathrm{~g}, 0.10 \mathrm{mmol})$ in one portion. The resulting solution was stirred overnight at RT and the solvent was then removed under reduced pressure.

The dark residue was filtered through a pad of silica gel (10:1 hexane:EtOAc) and then purified with two sequential flash chromatographic separations (12:1 hexane:EtOAc) to provide the title compound as a white crystalline solid (0.13 g, 40\%). ${ }^{1} \mathrm{H} \mathrm{NMR}\left(500 \mathrm{MHz}, \mathrm{CDCl}_{3}\right) \delta$ 7.65-7.58 $(\mathrm{m}, 4 \mathrm{H}), 7.44-7.33(\mathrm{~m}, 6 \mathrm{H}), 5.42(\mathrm{t}, J=9.2 \mathrm{~Hz}, 1 \mathrm{H}), 4.88(\mathrm{dd}, J=9.8,3.6 \mathrm{~Hz}, 1 \mathrm{H}), 4.06$ (dt, $J$ $=11.0,4.6 \mathrm{~Hz}, 1 \mathrm{H}), 4.01(\mathrm{td}, J=10.1,3.2 \mathrm{~Hz}, 1 \mathrm{H}), 2.95-2.88(\mathrm{~m}, 1 \mathrm{H}), 2.93(\mathrm{dd}, J=14.9,9.8$ $\mathrm{Hz}, 1 \mathrm{H}), 2.60(\mathrm{dd}, J=15.3,3.5 \mathrm{~Hz}, 1 \mathrm{H}), 2.28-2.22(\mathrm{~m}, 1 \mathrm{H}), 2.23(\mathrm{~s}, 3 \mathrm{H}), 1.58(\mathrm{~s}, 3 \mathrm{H})$. Spectral data matched those reported by Stocker and Turner. ${ }^{2,5}$

Representative procedure for boron-mediated 1,5-anti-aldol reactions : To a solution of the ketone (1.0 eq.) in $\mathrm{Et}_{2} \mathrm{O}\left(10 \mathrm{~mL}\right.$ per mmol of ketone) at $-78{ }^{\circ} \mathrm{C}$ was added $\mathrm{Et}_{3} \mathrm{~N}$ (3.2 eq.) followed by $\mathrm{Cy}_{2} \mathrm{BCl}$ (2.2 eq.). The resulting mixture was stirred at $-78{ }^{\circ} \mathrm{C}$ for $10 \mathrm{~min}$ and then warmed to $0{ }^{\circ} \mathrm{C}$ for $30 \mathrm{~min}$ and then cooled back to $-78{ }^{\circ} \mathrm{C}$. The aldehyde $(2.0$ eq. $)$ in $\mathrm{Et}_{2} \mathrm{O}$ (1 mL per mmol of aldehyde) was added dropwise. The reaction was stirred at $-78{ }^{\circ} \mathrm{C}$ for $1 \mathrm{~h}$ and then put into $-18{ }^{\circ} \mathrm{C}$ freezer overnight. The reaction was then quenched with $\mathrm{MeOH}$ (10 $\mathrm{mL}$ per mmol of ketone) and $\mathrm{pH} 7$ sodium phosphate buffer (10 $\mathrm{mL}$ per mmol of ketone) solution and stirred for $1 \mathrm{~h}$. The solution was extracted with $\mathrm{CH}_{2} \mathrm{Cl}_{2}(3 \times 10 \mathrm{~mL}$ per mmol of ketone) and the organic fraction was washed with sat. brine (10 $\mathrm{mL}$ per mmol of ketone) and dried with $\mathrm{MgSO}_{4}$. The solvent was removed under reduced pressure. Flash chromatography or preparative TLC was used to purify the product.

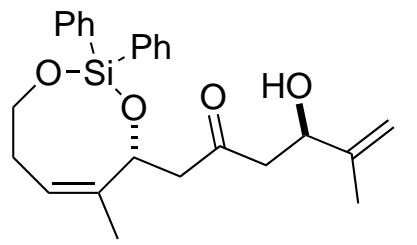

$\left[\left(4 R^{*}, 4^{\prime} R^{*}\right),(5 Z)\right]-2,2-D i p h e n y l-5-m e t h y l-4-\left(4^{\prime}-h y d r o x y-5 '-\right.$ methyl-2'-oxohex-5'-enyl)-7,8-dihydro- $4 H$-1,3-dioxa-2-silocine

(28a): An aldol reaction of $\mathbf{2}(30 \mathrm{mg}, 0.085 \mathrm{mmol})$ with methacrolein (0.014 mL, $12 \mathrm{mg}, 0.17 \mathrm{mmol})$ was carried out. Flash chromatography (10:1 hexane:EtOAc) provided (18 mg, 49\%, dr >99:1 anti:syn by ${ }^{1} \mathrm{H}$ NMR) as a colorless oil. ${ }^{1} \mathrm{H} \mathrm{NMR}\left(500 \mathrm{MHz}, \mathrm{CDCl}_{3}\right) \delta$ 7.64-7.57 (m, 4H), 7.44-7.33 (m, 6H), 5.44 $(\mathrm{t}, J=8.8 \mathrm{~Hz}, 1 \mathrm{H}), 4.99(\mathrm{~s}, 1 \mathrm{H}), 4.90(\mathrm{dd}, J=9.6,3.3 \mathrm{~Hz}, 1 \mathrm{H}), 4.85(\mathrm{~s}, 1 \mathrm{H}), 4.52(\mathrm{~d}, J=9.3 \mathrm{~Hz}$, $1 \mathrm{H}), 4.07-4.00(\mathrm{~m}, 2 \mathrm{H}), 3.01(\mathrm{~d}, J=2.2 \mathrm{~Hz}, 1 \mathrm{H}, \mathrm{OH}), 2.96(\mathrm{dd}, J=14.8,9.7 \mathrm{~Hz}, 1 \mathrm{H}), 2.93-2.85$ $(\mathrm{m}, 1 \mathrm{H}), 2.81(\mathrm{dd}, J=17.6,2,7 \mathrm{~Hz}, 1 \mathrm{H}), 2.74(\mathrm{dd}, J=17.7,9.3 \mathrm{~Hz}, 1 \mathrm{H}), 2.61(\mathrm{dd}, J=14.8$, $3.5 \mathrm{~Hz}, 1 \mathrm{H}), 2.28-2.24(\mathrm{~m}, 1 \mathrm{H}), 1.70(\mathrm{~s}, 3 \mathrm{H}), 1.59(\mathrm{~s}, 3 \mathrm{H}) .{ }^{13} \mathrm{C} \mathrm{NMR}\left(125 \mathrm{MHz}, \mathrm{CDCl}_{3}\right) \delta 210.0$, 145.6, 140.4, 134.5, 134.4, 133.27, 133.07, 130.2, 130.1, 127.9, 127.8, 125.0, 111.0, 71.3, 70.8, 64.3, 49.2, 49.1, 30.6, 21.1, 18.3. IR (neat) 3459, 3070, 2922, 1710, 1430, 1377, 1270, 1185, 1116, 972, 930, 717, 700. HRMS (ESI) calcd. for $\mathrm{C}_{25} \mathrm{H}_{30} \mathrm{NaO}_{4} \mathrm{Si}\left(\mathrm{M}+\mathrm{Na}^{+}\right) 445.1811$, found 445.1808 . 


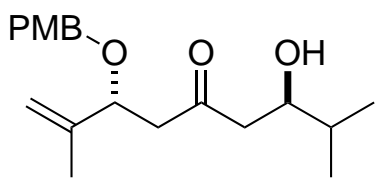

$\left(3 R^{*}, 7 R^{*}\right)$-7-Hydroxy-3-(4'-methoxybenzyloxy)-2,8-dimethylnonene-5-one (32b): An aldol reaction of (rac)-20 (30 mg, 0.12 mmol) with isobutyraldehyde $(0.02 \mathrm{~mL}, 17 \mathrm{mg}, 0.24 \mathrm{mmol})$ was carried out. Three sequential flash chromatographic separations (3:1 hexane:EtOAc) were used to provide $\mathbf{3 2 b}(9.0 \mathrm{mg}, 24 \%,>99: 1$ (the relative stereochemistry of the major product was assumed to be anti but not determined as the following two-step chemical transformation was not successful) by ${ }^{1} \mathrm{H} \mathrm{NMR}$ ) as a colorless oil. ${ }^{1} \mathrm{H}$ NMR (500 $\mathrm{MHz}, \mathrm{CDCl}_{3}$ ) $\delta 7.20(\mathrm{~d}, J=8.5 \mathrm{~Hz}, 2 \mathrm{H}), 6.86(\mathrm{~d}, J=8.5 \mathrm{~Hz}, 2 \mathrm{H}), 5.04(\mathrm{~s}, 1 \mathrm{H}), 5.00(\mathrm{~s}, 1 \mathrm{H}), 4.40(\mathrm{~d}, J=11.3$ $\mathrm{Hz}, 1 \mathrm{H}), 4.27(\mathrm{dd}, J=9.8,3.7 \mathrm{~Hz}, 1 \mathrm{H}), 4.20(\mathrm{~d}, J=11.1 \mathrm{~Hz}, 1 \mathrm{H}), 3.82-3.77(\mathrm{~m}, 1 \mathrm{H}), 3.79(\mathrm{~s}$, $3 \mathrm{H}), 2.87(\mathrm{dd}, J=15.3 \mathrm{~Hz}, 10.0,1 \mathrm{H}), 2.63(\mathrm{dd}, J=17.5,2.0 \mathrm{~Hz}, 1 \mathrm{H}), 2.46(\mathrm{dd}, J=17.5,9.8$ $\mathrm{Hz}, 1 \mathrm{H}), 2.42(\mathrm{dd}, J=15.4,3.8 \mathrm{~Hz}, 1 \mathrm{H}), 1.73(\mathrm{~s}, 3 \mathrm{H}), 1.68-1.61(\mathrm{~m}, 1 \mathrm{H}), 0.90(\mathrm{~d}, J=6.41 \mathrm{~Hz}$, $3 \mathrm{H}), 0.88(\mathrm{~d}, J=6.41 \mathrm{~Hz}, 3 \mathrm{H})$.

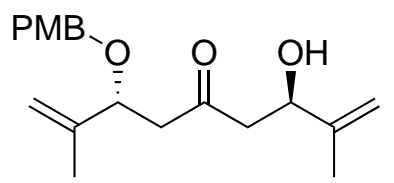

(3R,7R)-7-Hydroxy-3-(4'-methoxybenzyloxy)-2,8-dimethylnon-1,8-diene-5-one (32a): An aldol reaction of $(R)-20$ (50 mg, $0.20 \mathrm{mmol})$ with methacrolein $(0.033 \mathrm{~mL}, 28 \mathrm{mg}, 0.40 \mathrm{mmol})$ was carried out. Preparative TLC (3:1 hexane:EtOAc) provide 32a (30 mg, $47 \%$, dr > 99:1 anti:syn by ${ }^{1} \mathrm{H}$ NMR) as a colorless oil. ${ }^{1} \mathrm{H}$ NMR $\left(500 \mathrm{MHz}, \mathrm{CDCl}_{3}\right) \delta 7.21$ (d, $J=6.8 \mathrm{~Hz}, 2 \mathrm{H}) 6.87(\mathrm{~d}, J=8.8 \mathrm{~Hz}, 2 \mathrm{H}), 5.05(\mathrm{~s}, 1 \mathrm{H}), 5.02(\mathrm{~s}, 1 \mathrm{H}), 4.99(\mathrm{~s}, 1 \mathrm{H}), 4.86(\mathrm{~s}, 1 \mathrm{H})$, $4.51(\mathrm{~d}, J=9.8 \mathrm{~Hz}, 1 \mathrm{H}), 4.41(\mathrm{~d}, J=11.2 \mathrm{~Hz}, 1 \mathrm{H}), 4.29(\mathrm{dd}, J=9.3,2.4 \mathrm{~Hz}, 1 \mathrm{H}), 4.21(\mathrm{~d}$, $J=9.8 \mathrm{~Hz}, 1 \mathrm{H}), 3.81(\mathrm{~s}, 3 \mathrm{H}), 3.04(\mathrm{~s}, 1 \mathrm{H}), 2.88(\mathrm{ddd}, J=15.3,9.6,1.3 \mathrm{~Hz}, 1 \mathrm{H}), 2.74-2.70(\mathrm{~m}$, $1 \mathrm{H}), 2.62(\mathrm{dd}, J=17.6,9.5,1.5 \mathrm{~Hz}, 1 \mathrm{H}), 2.45(\mathrm{ddd}, J=15.1,3.5,1.5 \mathrm{~Hz}, 1 \mathrm{H}) 1.74(\mathrm{~s}, 3 \mathrm{H}), 1.71$ $(\mathrm{s}, 3 \mathrm{H}) .{ }^{13} \mathrm{C} \mathrm{NMR}\left(125 \mathrm{MHz}, \mathrm{CDCl}_{3}\right) \delta 209.6,159.2,147.6,143.2,130.0,129.6,114.3,113.8$, 79.1, 70.9, 70.1, 55.3, 49.0, 48.0, 18.4, 16.9. IR (neat) 3446, 2922, 2851, 1715, 1651, 1613, 1514, 1455, 1375, 1302, 1248, 1077, 1035, 903, 821. $[\alpha]_{D}^{22}+153.0$ (c 1.5, $\mathrm{CHCl}_{3}$ ). HRMS (ESI) calcd. for $\mathrm{C}_{19} \mathrm{H}_{26} \mathrm{NaO}_{4}\left(\mathrm{M}+\mathrm{Na}^{+}\right) 341.1729$, found 341.1722 .

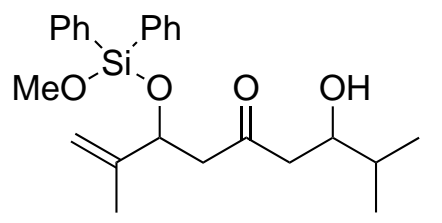

\section{3-(Methoxydiphenylsilyloxy)-7-hydroxy-2,8-dimethyl-} nonene-5-one (38b): An aldol reaction of ( $\mathrm{rac}$ )-19a (30 $\mathrm{mg}, 0.089$ $\mathrm{mmol})$ with isobutyraldehyde $(0.016 \mathrm{~mL}, 13 \mathrm{mg}, 0.18 \mathrm{mmol})$ was carried out. The crude ${ }^{1} \mathrm{H}$ NMR spectral data showed the presenece of $\mathbf{1 4 b}$. However, after two sequential episodes of flash chromatography, no product was isolated.

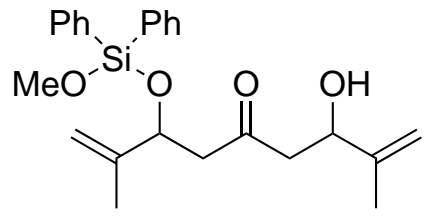

\section{3-(Methoxydiphenylsilyloxy)-7-hydroxy-2,8-dimethyl-} non-1,8-diene-5-one (38a): An aldol reaction of ( $r a c)-19 a(30$ $\mathrm{mg}, 0.089 \mathrm{mmol})$ with methacrolein $(0.015 \mathrm{~mL}, 13 \mathrm{mg}, 0.18 \mathrm{mmol})$ was carried out. The crude ${ }^{1} \mathrm{H}$ NMR spectral data showed the presence of 38a. After two sequential episodes of flash chromatography, no product was isolated. 

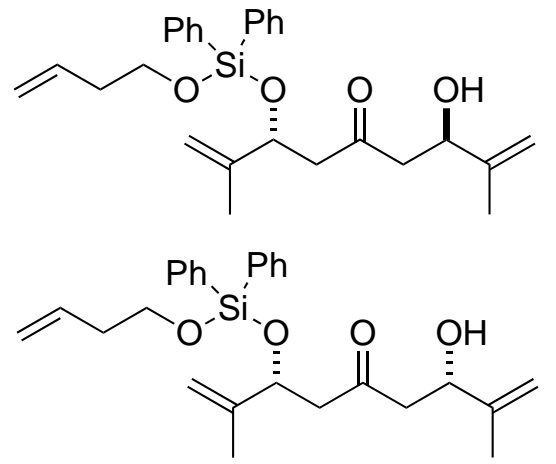

$\left(3 R^{*}, 7 R^{*}\right)$-3-(But-3-en-1-oxydiphenylsilyl-oxy)-7hydroxy-2,8-dimethyl-non-1,8-diene-5-one (anti-39a) and $\left(3 R^{*}, 7 S^{*}\right)-3$-(But-3-en-1-oxydiphenylsilyl-oxy)-7hydroxy-2,8-dimethyl-non-1,8-diene-5-one (syn-39a): An aldol reaction of ( $\mathrm{rac})-\mathbf{3}$ (100 $\mathrm{mg}, 0.26 \mathrm{mmol})$ with methacrolein $(0.04 \mathrm{~mL}, 36 \mathrm{mg}, 0.52 \mathrm{mmol})$ was carried out. Flash chromatography (10:1 hexane:EtOAc) provided 39a (30 mg, $26 \%, \mathrm{dr}=1: 1$ anti:syn by $\left.{ }^{1} \mathrm{H} \mathrm{NMR}\right)$ as a colorless oil. ${ }^{1} \mathrm{H}$ NMR $\left(500 \mathrm{MHz}, \mathrm{CDCl}_{3}\right) \delta$ 7.65-7.61(m, 4H), 7.46-7.41(m, $2 \mathrm{H}), 7.40-7.35(\mathrm{~m}, 4 \mathrm{H}), 5.86-5.77(\mathrm{~m}, 1 \mathrm{H}), 5.09-5.02(\mathrm{~m}, 2 \mathrm{H}), 4.96(\mathrm{~s}, 1 \mathrm{H}), 4.93(\mathrm{~s}, 1 \mathrm{H}), 4.84$ $(\mathrm{s}, 1 \mathrm{H}), 4.81-4.78(\mathrm{~m}, 2 \mathrm{H}), 4.42-4.39(\mathrm{~m}, 1 \mathrm{H}), 3.78$ (app. td, $J=6.8,1.0 \mathrm{~Hz}, 2 \mathrm{H}), 2.95-2.90$ $(\mathrm{m}, 1 \mathrm{H}), 2.87-2.81(\mathrm{~m}, 1 \mathrm{H}), 2.61-2.55(\mathrm{~m}, 3 \mathrm{H}), 2.35-2.31(\mathrm{~m}, 2 \mathrm{H}), 1.72(\mathrm{~s}, 3 \mathrm{H}), 1.69(\mathrm{~s}, 3 \mathrm{H}) .{ }^{13} \mathrm{C}$ NMR $\left(125 \mathrm{MHz}, \mathrm{CDCl}_{3}\right) \delta 209.2,209.1,145.6,145.3,135.0,132.6,132.5,132.4,130.37,130.35$, 130.32, 130.30, 127.8, 127.72, 127.71, 116.6, 112.4, 112.36, 111.11, 111.08, 73.1, 73.0, 70.9, 70.7, $62.8,50.2,50.0,49.0,48.7,36.8,18.34,18.28,17.4$. IR (neat) 3072, 2921, 1709, 1429, 1374, 1262, 1115, 1079, 904, 740, 718, 700. HRMS (ESI) calcd. for $\mathrm{C}_{27} \mathrm{H}_{34} \mathrm{O}_{4} \mathrm{NaSi},\left(\mathrm{M}+\mathrm{Na}^{+}\right) 473.2124$, found 473.2117 .

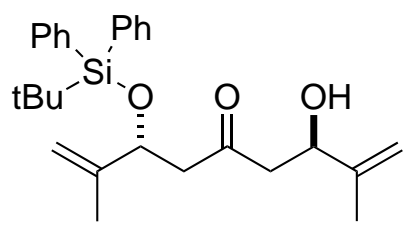

$\left(3 R^{*}, 7 R^{*}\right)-3-($ tert-Butyldiphenylsilyloxy)-7-hydroxy-2,8dimethyl-non-1,8-diene-5-one (anti-40a) and $\left(3 R^{*}, 7 S^{*}\right)-3-($ tertButyldiphenylsilyloxy)-7-hydroxy-2,8-dimethyl-non-1,8-diene5-one (syn-40a): An aldol reaction of ( $\mathrm{rac})-\mathbf{5}$ (56 $\mathrm{mg}, 0.15 \mathrm{mmol})$ with methacrolein $(0.02 \mathrm{~mL}, 21 \mathrm{mg}, 0.30 \mathrm{mmol})$ was carried out.<smiles>C=C(C)[C@H](CC(=O)C[C@@H](O)C(=C)C)O[Si](c1ccccc1)(c1ccccc1)c1ccccc1</smiles>
Flash chromatography (10:1 hexane:EtOAc) provided 40a (33 mg, $51 \%, \mathrm{dr}=1.5: 1$ anti:syn by $\left.{ }^{1} \mathrm{H} \mathrm{NMR}\right)$ as a colorless oil. ${ }^{1} \mathrm{H}$ NMR $\left(500 \mathrm{MHz}, \mathrm{CDCl}_{3}\right.$, major $=0.67 \mathrm{H}$, minor $\left.=0.33 \mathrm{H}\right) \delta 7.68-7.64(\mathrm{~m}$, $4 \mathrm{H}), 7.46-7.42(\mathrm{~m}, 2 \mathrm{H}), 7.40-7.35(\mathrm{~m}, 4 \mathrm{H}), 4.94(\mathrm{~s}, 0.33 \mathrm{H}), 4.92(\mathrm{~s}$, $0.67 \mathrm{H}), 4.82-4.69($ complex m, $3 \mathrm{H}+0.67 \mathrm{H}), 4.62(\mathrm{t}, J=6.2 \mathrm{~Hz}, 0.33 \mathrm{H}), 4.59(\mathrm{t}, J=6 \mathrm{~Hz}$, $0.67 \mathrm{H}), 4.35-4.33(\mathrm{~m}, 0.33 \mathrm{H}), 2.88$ (br. $1 \mathrm{H}, \mathrm{OH}), 2.67-2.41(\mathrm{~m}, 4 \mathrm{H}), 1.70-1.67(\mathrm{~m}, 6 \mathrm{H}), 1.06(\mathrm{~s}$, $9 \mathrm{H}) .{ }^{13} \mathrm{C} \mathrm{NMR}\left(125 \mathrm{MHz}, \mathrm{CDCl}_{3}\right) \delta 209.2,145.5,136.0,135.9,133.84,133.78,133.26,133.17$, $129.80,129.75,129.72,127.60,127.57,127.40,127.45,112.43,112.41,111.07,111.05,73.54$, $73.48,70.70,70.68,50.31,50.27,48.79,48.52,29.7,26.97,19.32,19.30,18.33,18.27,17.29$, 17.26. IR (neat) 3488, 3073, 2929, 2857, 1709, 1651, 1590, 1428, 1391, 1373, 1250, 1110, 904, 822, 734, 702. HRMS (ESI) calcd. for $\mathrm{C}_{27} \mathrm{H}_{36} \mathrm{NaO}_{3} \mathrm{Si}\left(\mathrm{M}+\mathrm{Na}^{+}\right)$459.2331, found 459.2325.

Representative procedure for the Evans-Chapman-Carreira reduction of aldol adducts: To a solution of MeCN (4 mL per mmol of ketone) and AcOH (12 mL per mmol of ketone) was added $\mathrm{Me}_{4} \mathrm{NBH}(\mathrm{OAc})_{3}$ (8 eq.) and the resulting solution was stirred at RT for 30 min. The reaction mixture was then cooled to $-10{ }^{\circ} \mathrm{C}$ and a solution of the ketone (1 eq.) in MeCN ( $8 \mathrm{~mL}$ per mmol of ketone) was added dropwise to the reaction mixture. The reaction was stirred at the same temperature for $2 \mathrm{~h}$ and then maintained at $4{ }^{\circ} \mathrm{C}$ overnight before be- 
ing quenched with sat. sodium potassium tartrate solution ( $25 \mathrm{~mL}$ per mmol of ketone) and partitioned with hexane:EtOAc (1:1) (50 $\mathrm{mL}$ per mmol of ketone). The resulting mixture was washed with sat. $\mathrm{NaHCO}_{3}(25 \mathrm{~mL}$ per mmol of ketone) and the aqueous phase was extracted with EtOAc ( $3 \times 50 \mathrm{~mL}$ per mmol of ketone). The organic fractions were combined, dried with $\mathrm{MgSO}_{4}$ and the solvent was removed with reduced pressure. Flash chromatography was used to purify the compound.

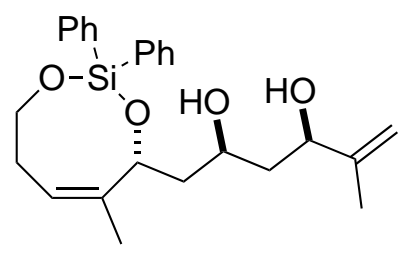

$\left[\left(4 R^{*}, 2^{\prime} S^{*}, 4^{\prime} R^{*}\right),(5 Z)\right]-2,2-D i p h e n y l-5-m e t h y l-4-\left(2^{\prime}, 4 '-\right.$ dihydroxy-5'-methyl-hex-5'-enyl)-7,8-dihydro-4H-1,3-dioxa-2silocine (syn-31) and $\left[\left(4 R^{*}, 2^{\prime} R^{*}, 4^{\prime} R^{*}\right),(5 Z)\right]-2,2-D i p h e n y l-5$ methyl-4-(2',4'- dihydroxy-5'-methyl-hex-5'-enyl)-7,8-dihydro4H-1,3-dioxa-2-silocine (anti-31): An Evans-Chapman-Carreira<smiles>C=C(C)C(O)C[C@H](O)C[C@H]1O[Si](c2ccccc2)(c2ccccc2)OCCC=C1C</smiles>
reduction of ketone $\mathbf{2 8 \mathbf { a }}(12 \mathrm{mg}, 0.028 \mathrm{mmol})$ was carried out. Flash chromatography (5:1 hexane:EtOAc) provided 31 (5.0 mg, 43\%, dr $=3: 1$ syn:anti by ${ }^{1} \mathrm{H}$ NMR $)$ as a colorless oil. ${ }^{1} \mathrm{H}$ NMR $(500 \mathrm{MHz}$, $\mathrm{CDCl}_{3}$, major $=0.75 \mathrm{H}$, minor $\left.=0.25 \mathrm{H}\right) \delta 7.65-7.58(\mathrm{~m}, 4 \mathrm{H}), 7.46-$ $7.34(\mathrm{~m}, 6 \mathrm{H}), 5.48-5.54(\mathrm{~m}, 0.25 \mathrm{H}) 5.39(\mathrm{t}, J=8.9 \mathrm{~Hz}, 0.75 \mathrm{H}), 5.06$ $(\mathrm{s}, 0.75 \mathrm{H}), 5.01(\mathrm{~s}, 0.25 \mathrm{H}), 4.90(\mathrm{~s}, 0.25 \mathrm{H}), 4.87(\mathrm{~s}, 0.75 \mathrm{H}), 4.69-4.64(\mathrm{~m}, 0.25 \mathrm{H}) 4.58($ app. d, J $=10.5 \mathrm{~Hz}, 0.75 \mathrm{H}), 4.39-4.35(\mathrm{~m}, 1 \mathrm{H}), 4.26-4.22(\mathrm{~m}, 1 \mathrm{H}), 4.16-4.13(\mathrm{~m}, 1 \mathrm{H}) 4.07(\mathrm{~s}, 0.75 \mathrm{H}, \mathrm{OH})$ 4.05-4.03 (m, 1H), 3.16 (br. s, $1 \mathrm{H}, \mathrm{OH}), 2.97-2.94(\mathrm{~m}, 1 \mathrm{H}), 2.27-2.24(\mathrm{~m}, 1 \mathrm{H}), 2.05$ (dt, $J=$ $14.3,10.3 \mathrm{~Hz}, 1 \mathrm{H}) 1.76-1.57(\mathrm{~m}, 3 \mathrm{H}), 1.73(\mathrm{~s}, 3 \mathrm{H}), 1.53(\mathrm{~s}, 3 \mathrm{H}) \cdot{ }^{13} \mathrm{C} \mathrm{NMR}\left(125 \mathrm{MHz}, \mathrm{CDCl}_{3}\right) \delta$ 147.4, 141.3, 134.4, 134.3, 133.0, 132.9, 130.3, 128.0, 127.9, 124.7, 110.3, 72.5, 70.4, 70.1, 64.3, 41.6, 41.1, 30.7, 29.7, 21.3, 18.6. IR (neat) 3404, 3070, 2920, 2875, 1429, 1378, 1261, 1169, 1116, 900, 717, 700. HRMS (ESI) calcd. for $\mathrm{C}_{25} \mathrm{H}_{23} \mathrm{NaO}_{4} \mathrm{Si}\left(\mathrm{M}+\mathrm{Na}^{+}\right)$447.1968, found 447.1967.

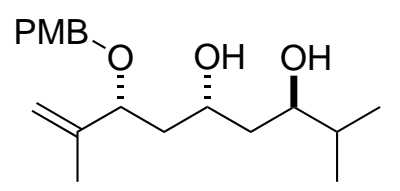

$\left(3 R^{*}, 5 R^{*}, 7 R^{*}\right)-5,7-D i h y d r o x y-3-\left(4^{\prime}-m e t h o x y b e n z y l o x y\right)-2,8-$ dimethyl-nonene (36): An Evans-Chapman-Carreira reduction of ketone 32b (10 mg, $0.031 \mathrm{mmol}$ ) was carried out. Flash chromatography (3:1 hexane:EtOAc) provided $\mathbf{3 6}(10 \mathrm{mg},>99 \%$, dr $>99: 1$ by ${ }^{1} \mathrm{H}$ NMR $) .{ }^{1} \mathrm{H}$ NMR $\left(500 \mathrm{MHz}, \mathrm{CDCl}_{3}\right) \delta 7.23(\mathrm{~d}, J=8.6 \mathrm{~Hz}, 2 \mathrm{H}), 6.88(\mathrm{~d}, J=8.8 \mathrm{~Hz}, 2 \mathrm{H})$, $5.00(\mathrm{~s}, 2 \mathrm{H}), 4.48(\mathrm{~d}, J=11.3 \mathrm{~Hz}, 1 \mathrm{H}), 4.21(\mathrm{~d}, J=11.3 \mathrm{~Hz}, 1 \mathrm{H}), 4.17-4.13(\mathrm{~m}, 1 \mathrm{H}), 4.11$ (br. $\mathrm{s}, 1 \mathrm{H}), 4.06(\mathrm{dd}, J=10.3,2.9 \mathrm{~Hz}, 1 \mathrm{H}), 3.81(\mathrm{~s}, 3 \mathrm{H}), 3.67-3.64(\mathrm{~m}, 1 \mathrm{H}), 1.75(\mathrm{~s}, 3 \mathrm{H}), 1.69-1.58$ $(\mathrm{m}, 3 \mathrm{H}), 1.54-1.50(\mathrm{~m}, 2 \mathrm{H}), 0.93(\mathrm{~d}, J=6.8,3 \mathrm{H}), 0.89(\mathrm{~d}, J=6.8,3 \mathrm{H})$.

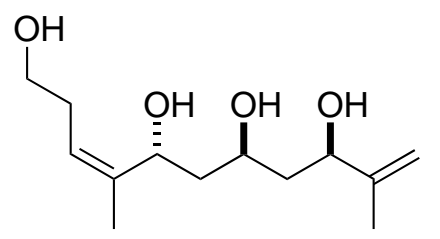

$\left[\left(3 R^{*}\right),\left(5 R^{*}\right),\left(7 R^{*}\right)\right]-2,8-$ Dimethyl-3,5,7,11,-tetrahydroxyundeca-1,8-diene (29): To a solution of $\mathbf{3 1}$ (10 $\mathrm{mg}, 0.024 \mathrm{mmol})$ in THF $(4 \mathrm{~mL})$ was added $\mathrm{Bu}_{4} \mathrm{NF}$ as a $2 \mathrm{M}$ solution in THF $(0.024$ $\mathrm{mL}, 0.048 \mathrm{mmol})$ at $0{ }^{\circ} \mathrm{C}$. The reaction was monitored by TLC. After TLC showed completion of reaction, the solvent was removed under reduced pressure. The resulting red oil was loaded on a HP20 column with cyclic loading (diluting the eluent within equal quantity of water and then loaded on the HP20 column again 
and the process was repeated three times) and then the column was washed with $\mathrm{H}_{2} \mathrm{O}(10 \mathrm{~mL})$, $\mathrm{MeOH}: \mathrm{H}_{2} \mathrm{O}$ 1:1 $(10 \mathrm{~mL})$ and $\mathrm{MeOH}(10 \mathrm{~mL})$. The crude product eluted in the $\mathrm{MeOH}$ fraction and then purified with flash chromatography $\left(12: 1 \mathrm{CH}_{2} \mathrm{Cl}_{2}: \mathrm{MeOH}\right)$. The resulting product was further purified with preparative TLC $\left(5: 1 \mathrm{CH}_{2} \mathrm{Cl}_{2}: \mathrm{MeOH}\right)$ to afford a pale yellow oil $(2 \mathrm{mg}$, $34 \%) .{ }^{1} \mathrm{H}$ NMR $\left(600 \mathrm{MHz}, \mathrm{CD}_{3} \mathrm{OD}\right) \delta 5.30(\mathrm{t}, J=7.1 \mathrm{~Hz}, 1 \mathrm{H}), 5.00(\mathrm{~s}, 1 \mathrm{H}) 4.82-4.80(\mathrm{~m}, 2 \mathrm{H})$, $4.23(\mathrm{dd}, J=7.8,4.8 \mathrm{~Hz}, 1 \mathrm{H}), 3.79$ (app. ddd, $J=12.5,8.4,4.2 \mathrm{~Hz}, 1 \mathrm{H}$ ), 3.65 (br. s, $1 \mathrm{H}$, $\mathrm{OH}), 3.60-3.50(\mathrm{~m}, 4 \mathrm{H}, 2 \mathrm{H}+2 \mathrm{OH}), 2.34$ (app. q, $J=6.6 \mathrm{~Hz}, 2 \mathrm{H}), 1.72-1.71(\mathrm{~m}, 1 \mathrm{H}) 1.70(\mathrm{~s}$, $3 \mathrm{H}) 1.69(\mathrm{~s}, 3 \mathrm{H}), 1.68-1.60(\mathrm{~m}, 3 \mathrm{H}) .{ }^{13} \mathrm{C} \mathrm{NMR}\left(600 \mathrm{MHz}, \mathrm{CD}_{3} \mathrm{OD}\right) \delta 149.5,139.6,125.4,110.7$, 73.0, 68.4, 67.5, 62.7, 44.3, 43.0, 33.1, 18.1, 17.8. IR (neat) 3342, 2923, 2854, 1721, 1567, 1454, 1377, 1053, 898. HRMS (ESI) calcd. for $\mathrm{C}_{13} \mathrm{H}_{24} \mathrm{NaO}_{4}\left(\mathrm{M}+\mathrm{Na}^{+}\right)$267.1572, found 267.1573.

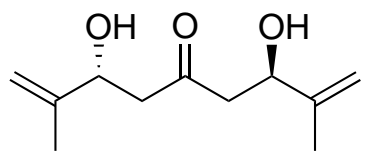

(3R,7R)-3,7-Dihydroxy-non-1,8-diene-5-one (33a): To a stirring solution of $\mathbf{3 2 a}(25 \mathrm{mg}, 0.079 \mathrm{mmol})$ in $\mathrm{CH}_{2} \mathrm{Cl}_{2}(3.5 \mathrm{~mL})$ was added $\mathrm{pH} 7$ buffer solution and DDQ in $1 \mathrm{M}$ solution of $\mathrm{CH}_{2} \mathrm{Cl}_{2}(0.32 \mathrm{~mL}, 0.32$ $\mathrm{mmol}$ ) in four portions, each separated by $15 \mathrm{~min}$ and TLC was used to monitor the reaction before adding the next portion of DDQ solution. After the addition of DDQ, the reaction was allowed to proceed for $2 \mathrm{~h}$ and then diluted with hexane/EtOAc 1:1 (40 mL), washed with $\mathrm{NaHCO}_{3}(2 \times 20 \mathrm{~mL})$ and brine $(20 \mathrm{~mL})$. The organic layer was dried with $\mathrm{MgSO}_{4}$. Flash chromatography (1:1 hexane:EtOAc) was used to purify 33a to provide a pale yellow oil (11 mg, $69 \%) .{ }^{1} \mathrm{H}$ NMR $\left(500 \mathrm{MHz}, \mathrm{CDCl}_{3}\right) \delta 5.03(\mathrm{~s}, 2 \mathrm{H}), 4.88(\mathrm{~s}, 2 \mathrm{H}), 4.55(\mathrm{~d}, J=8.5 \mathrm{~Hz}, 2 \mathrm{H}), 2.71$ $(\mathrm{m}, 4 \mathrm{H}), 1.75(\mathrm{~s}, 6 \mathrm{H}) .{ }^{13} \mathrm{C} \mathrm{NMR}\left(125 \mathrm{MHz}, \mathrm{CDCl}_{3}\right) \delta 209.4,148.5,110.5,71.9,50.2,18.4$. IR (neat) $3418,2922,2852,1707,1541,1445,1373,1302,1064,1033,897,808 .[\alpha]_{D}^{23}+15.4$ (c 0.54 , $\left.\mathrm{CHCl}_{3}\right)$. HRMS (ESI) calcd. for $\mathrm{C}_{11} \mathrm{H}_{18} \mathrm{NaO}_{3},\left(\mathrm{M}+\mathrm{Na}^{+}\right) 221.1154$, found 221.1153.

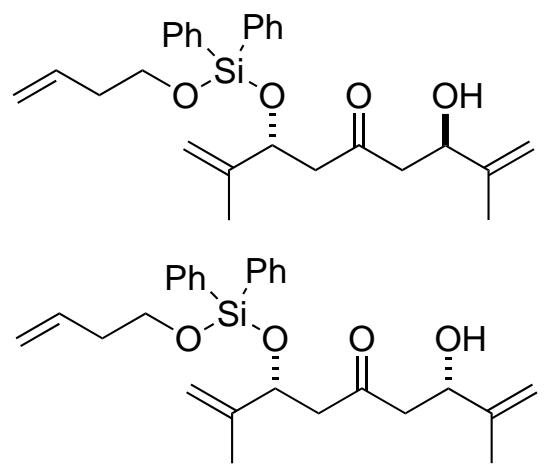

$\left(3 R^{*}, 7 R^{*}\right)$-3-(But-3-en-1-oxydiphenylsilyloxy)-7hydroxy-2,8-dimethyl-non-1,8-diene-5-one (anti-39a) and $\left(3 R^{*}, 7 S^{*}\right)$-3-(But-3-en-1-oxydiphenylsilyloxy)-7hydroxy-2,8-dimethyl-non-1,8-diene-5-one (syn-39a): A $2 \mathrm{M}$ solution of $\mathrm{BuLi}(0.16 \mathrm{~mL}, 0.31 \mathrm{mmol})$ in cyclohexane was added to $i \operatorname{Pr}_{2} \mathrm{NH}(0.043 \mathrm{~mL}, 30 \mathrm{mg}, 0.13 \mathrm{mmol})$ in THF $(1.2 \mathrm{~mL})$ at $-78{ }^{\circ} \mathrm{C}$. After $30 \mathrm{~min},(\mathrm{rac})-\mathbf{3 a}(100 \mathrm{mg}, 0.26$ $\mathrm{mmol}$ ) was added slowly and the reaction was run for $50 \mathrm{~min}$ at $-78{ }^{\circ} \mathrm{C}$, then freshly distilled methacrolein $(0.040 \mathrm{~mL}, 36$ $\mathrm{mg}, 0.52 \mathrm{mmol}$ ) was added dropwise. The reaction was stirred for $30 \mathrm{~min}$ and then quenched with sat. $\mathrm{NH}_{4} \mathrm{Cl}$ solution. The mixture was warmed to $\mathrm{RT}$ and the aqueous layer was extracted with $\mathrm{CH}_{2} \mathrm{Cl}_{2}(3 \times 5 \mathrm{~mL})$. The organic fractions were combined and washed with sat. brine $(10 \mathrm{~mL})$, dried with $\mathrm{MgSO}_{4}$ and the solvent was removed with reduced pressure. The resulting yellow oil was purified with flash chromatography (10:1 hexane:EtOAc) to give a pale yellow oil (30mg, 26\%, dr = 1:1 anti:syn by $\left.{ }^{1} \mathrm{H} \mathrm{NMR}\right) .{ }^{1} \mathrm{H} \mathrm{NMR}\left(500 \mathrm{MHz}, \mathrm{CDCl}_{3}\right) \delta 7.65-7.61(\mathrm{~m}$, $4 \mathrm{H})$, 7.46-7.41(m, 2H), 7.40-7.35(m, 4H), 5.86-5.77 (m, 1H), 5.09-5.02 (m, 2H), $4.96(\mathrm{~s}, 1 \mathrm{H})$, $4.93(\mathrm{~s}, 1 \mathrm{H}), 4.84(\mathrm{~s}, 1 \mathrm{H}), 4.80(\mathrm{~d}, 2 \mathrm{H}), 4.42-4.39(\mathrm{~m}, 1 \mathrm{H}), 3.78$ (app. td, $J=6.8,1 \mathrm{~Hz}, 2 \mathrm{H})$, 
2.95-2.90 (m, 1H), 2.87-2.81 (m, 1H), 2.61-2.55 (m, 3H), 2.35-2.31 (m, 2H), $1.72(\mathrm{~s}, 3 \mathrm{H}), 1.69(\mathrm{~s}$, $3 \mathrm{H})$. Spectral data matched those of anti- and syn-39a obtained by the boron-mediated aldol reaction of $(\mathrm{rac})$-4e with methacrolein described above.

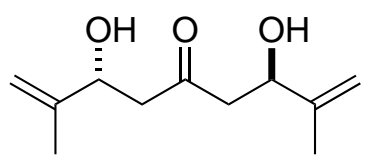

$(3 R, 7 R)$-3,7-Dihydroxy-non-1,8-diene-5-one (33a from deprotection of $40 \mathrm{a})$ : To a solution of $40 \mathrm{a}(15 \mathrm{mg}, 0.034 \mathrm{mmol})$ in THF $(0.2 \mathrm{~mL})$ was added a $2 \mathrm{M}$ solution of TBAF in THF $(0.07 \mathrm{~mL}$, $0.14 \mathrm{mmol}$ ) at $0{ }^{\circ} \mathrm{C}$. The reaction was monitored by TLC. After TLC showed a completion of reaction in $4 \mathrm{~h}$, the solvent was removed under reduced pressure. The resulting red oil was loaded on a HP20 column with cyclic loading (diluting the eluent with equal quantity of water and then loaded on the HP20 column againg and the process was repeated three times) and then the column was washed with $\mathrm{H}_{2} \mathrm{O}(10 \mathrm{~mL}), \mathrm{MeOH}: \mathrm{H}_{2} \mathrm{O}$ 1:1 $(10 \mathrm{~mL}), \mathrm{MeOH}(10 \mathrm{~mL})$. The crude product came out in the $\mathrm{MeOH}$ fraction and the major component matched the one synthesized from deprotection of 32a (crude yield was quantitative but no more purification was done).

Representative procedure for titanium tetrachloride mediated aldol reaction: To a solution of ketone (1 eq.) in $\mathrm{CH}_{2} \mathrm{Cl}_{2}(0.25 \mathrm{M})$ at $-10{ }^{\circ} \mathrm{C}$ was added $\mathrm{TiCl}_{4}$ (1.5 eq.) followed by $\mathrm{iPr}_{2} \mathrm{NEt}$ (1.8 eq.) and the reaction mixture was stirred at the same temperature for $1 \mathrm{~h}$. The solution was then cooled to $-78{ }^{\circ} \mathrm{C}$ and aldehyde (2.0 eq.) or aldehyde in a solution of $\mathrm{CH}_{2} \mathrm{Cl}_{2}(1$ $\mathrm{mL}$ per mmol of aldehyde) was added. The reaction was run at $-78^{\circ} \mathrm{C}$ for $1 \mathrm{~h}$ and then warmed to $0{ }^{\circ} \mathrm{C}$ before quenching with $\mathrm{pH}=7$ phosphate buffer solution (10 $\mathrm{mL}$ per mmol of ketone). After $1 \mathrm{~h}$, the solution was extracted with $\mathrm{CH}_{2} \mathrm{Cl}_{2}(3 \times 10 \mathrm{~mL}$ per mmol of ketone) and the organic fraction was dried with $\mathrm{MgSO}_{4}$. The solvent was removed with reduced pressure and then flash chromatography was used to purify the aldol product.
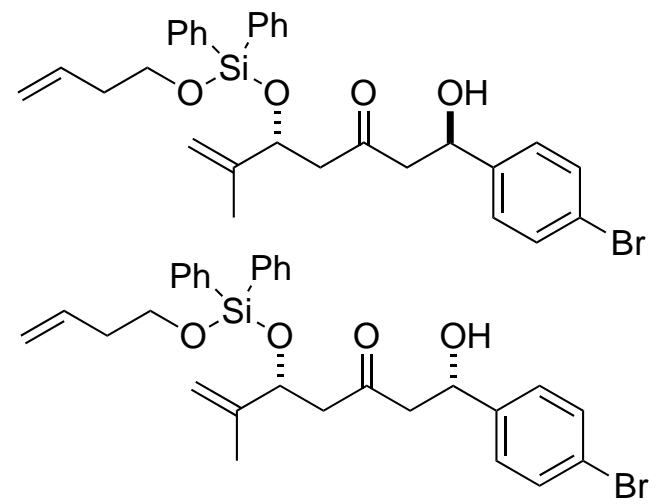

$\left(4 R^{*}\right),\left(7 R^{*}\right)-3-(B u t-3 '-e n-1 '-o x y d i p h e n y l s i l y l-$ oxy)-7-hydroxy-7-(4'-bromobenzyl)-2-methylheptene-5-one (anti-39d) and $\left(4 R^{*}\right),\left(7 S^{*}\right)-3$-(But3'-en-1'-oxydiphenylsilyloxy)-7-hydroxy-7-(4'bromobenzyl)-2-methylheptene-5-one (syn-39d): A $\mathrm{TiCl}_{4}$ mediated aldol reaction of ( rac)-3 (50 mg, $0.13 \mathrm{mmol}$ ) and 4-bromobenzyl aldehyde (48 $\mathrm{mg}, 0.26$ mmol) was carried out. Flash chromatography (10:1 hexane:EtOAc) provided 39d (74 mg, >99\%, dr =

1.3:1 anti:syn by $\left.{ }^{1} \mathrm{H} \mathrm{NMR}\right)$ as a pale yellow oil. ${ }^{1} \mathrm{H} \mathrm{NMR}\left(500 \mathrm{MHz}, \mathrm{CDCl}_{3}\right.$, major $=0.57 \mathrm{H}$, minor $=0.43 \mathrm{H}) \delta 7.59-7.04(\mathrm{~m}, 14 \mathrm{H}), 5.77-5.68(\mathrm{~m}, 1 \mathrm{H}), 5.00(\mathrm{~m}, 1 \mathrm{H}), 4.97-4.96(\mathrm{~m}, 1 \mathrm{H}), 4.94-$ $4.18(\mathrm{~m}, 1 \mathrm{H}), 4.85(\mathrm{~s}, 1 \mathrm{H}), 4.73(\mathrm{~s}, 1 \mathrm{H}), 4.70-4.68(\mathrm{~m}, 1 \mathrm{H}), 3.65(\mathrm{t}, J=6.4 \mathrm{~Hz}, 2 \mathrm{H}), 2.82-2.76$ $(\mathrm{m}, 1 \mathrm{H}), 2.75-2.70(\mathrm{~m}, 2 \mathrm{H}), 2.50-2.43(\mathrm{~m}, 1 \mathrm{H}), 2.25-2.21(\mathrm{~m}, 4 \mathrm{H}), 1.63(\mathrm{~s}, 6 \mathrm{H}) .{ }^{13} \mathrm{C}$ NMR $(125$ $\left.\mathrm{MHz}, \mathrm{CDCl}_{3}\right) \delta 209.0,145.3,141.6,135.0,134.3,131.7,131.6,131.5,130.32,127.9,127.8,127.4$, $116.6,73.2,73.1,69.5,69.0,62.8,52.0,50.0,48.4,36.9,32.0,29.7,18.0,17.4$. 

(104 mg, $1 \mathrm{mmol}$ ) at $0{ }^{\circ} \mathrm{C}$. The mixture was further stirred for $45 \mathrm{~min}$ at $0{ }^{\circ} \mathrm{C}$ followed by the addition of 4-methoxybenzyl chloride dropwise. The reaction was warmed to RT and stirred overnight. The solution was then quenched with $\mathrm{MeOH}(5 \mathrm{~mL})$ and ice water $(5 \mathrm{~mL})$ and then extracted with $\mathrm{Et}_{2} \mathrm{O}(3 \times 10 \mathrm{~mL})$. The organic fractions were combined and washed with $\mathrm{NaHCO}_{3}(3 \times 5 \mathrm{~mL})$, dried with $\mathrm{MgSO}_{4}$ and removed under reduced pressure. The yellow oil was purified with flash chromatography (10:1, hexane:EtOAc) to afford 56 (120 mg, 54\%) as a colorless oil. ${ }^{1} \mathrm{H}$ NMR $\left(500 \mathrm{MHz}, \mathrm{CDCl}_{3}\right) \delta$ 7.27-7.23 (m, 2H), 6.89-6.88 (m, 2H), $4.44(\mathrm{~s}, 2 \mathrm{H}), 3.81$ $(\mathrm{s}, 3 \mathrm{H}), 3.45(\mathrm{~s}, 2 \mathrm{H}), 3.30(\mathrm{~s}, 2 \mathrm{H}), 0.92(\mathrm{~s}, 6 \mathrm{H})$. Spectral data matched those reported by Turner. ${ }^{2}$<smiles>CCOC(=O)C(C)(C)C(=O)NC</smiles>

Ethyl 2,2-dimethyl-3-oxo-decanoate (57): To a solution of diisopropylamine $(0.29 \mathrm{~mL}, 0.200 \mathrm{~g}, 2.0 \mathrm{~mol})$ in THF $(3.5 \mathrm{~mL})$ was added a $2 \mathrm{M}$ solution of BuLi in cyclohexane $(1 \mathrm{~mL}, 2 \mathrm{mmol})$ at $-78{ }^{\circ} \mathrm{C}$. The resulting mixture was stirred for $15 \mathrm{~min}$ and then ethyl 2-methylpropanoate $(0.23 \mathrm{~g}, 0.27 \mathrm{~mL}, 2.0 \mathrm{mmol})$ was added dropwise and stirred for $45 \mathrm{~min}$ at the same temperature. Octanoyl chloride $(0.38$ $\mathrm{mL}, 0.360 \mathrm{~g}, 2.2 \mathrm{mmol}$ ) was added and the resulting solution was stirred for $40 \mathrm{~min}$. The reaction was quenched with sat. aq. $\mathrm{NH}_{4} \mathrm{Cl}(10 \mathrm{~mL})$ and extracted with $\mathrm{Et}_{2} \mathrm{O}(3 \mathrm{x} 10 \mathrm{~mL})$ then dried with $\mathrm{MgSO}_{4}$. The solvent was removed under reduced pressure. The crude oil was purified with flash chromatography (20:1 hexane:EtOAc) to provide a colorless oil $\mathbf{5 7}$ (0.109 g, 22\%). ${ }^{1} \mathrm{H} \mathrm{NMR}\left(500 \mathrm{MHz}, \mathrm{CDCl}_{3}\right) \delta 4.18(\mathrm{q}, J=7.1 \mathrm{~Hz}, 2 \mathrm{H}), 2.44(\mathrm{t}, J=7.3 \mathrm{~Hz}, 2 \mathrm{H}), 1.36(\mathrm{~s}, 6 \mathrm{H})$, $1.30-1.25(\mathrm{~m}, 13 \mathrm{H}), 0.88(\mathrm{t}, J=6.9 \mathrm{~Hz}, 3 \mathrm{H}) .{ }^{13} \mathrm{C} \mathrm{NMR}\left(125 \mathrm{MHz}, \mathrm{CDCl}_{3}\right) \delta 208.2,173.8,61.2$, 55.6, 38.0, 31.7, 29.11, 29.08, 23.9, 22.6, 21.9, 14.1, 14.0. IR (neat) 2927, 2856, 1713, 1467, 1385, $1366,1262,1147,1122,1065,1028,859,807$. HRMS (ESI) calcd. for $\mathrm{C}_{14} \mathrm{H}_{26} \mathrm{NaO}_{3}\left(\mathrm{M}+\mathrm{Na}^{+}\right)$ 265.1780 , found 265.1785 .<smiles>CCC(O)C(C)(C)CO</smiles>

2,2-Dimethyldecan-1,3-diol (58): To a suspension of $\mathrm{LiAlH}_{4}(44 \mathrm{mg}$, $1.15 \mathrm{mmol})$ in $\mathrm{Et}_{2} \mathrm{O}(2 \mathrm{~mL})$ was added $\mathbf{5 7}(90 \mathrm{mg}, 0.37 \mathrm{mmol})$ at $0{ }^{\circ} \mathrm{C}$. The reaction mixture was stirred overnight at $\mathrm{RT}$ and then quenched with sat. sodium potassium tatrate solution $(10 \mathrm{~mL})$ and extracted with $\mathrm{Et}_{2} \mathrm{O}(3 \times 10 \mathrm{~mL})$. The organic fractions were combined and washed with brine $(10 \mathrm{~mL})$ and dried with $\mathrm{MgSO}_{4}$. The oil was purified with flash chromatography to provide a colorless oil (43 mg, 58\%). ${ }^{1} \mathrm{H} \mathrm{NMR}(500 \mathrm{MHz}$, $\left.\mathrm{CDCl}_{3}\right) \delta 3.59-3.56(\mathrm{~m}, 1 \mathrm{H}), 3.49-3.46(\mathrm{~m}, 2 \mathrm{H}), 1.57-1.48(\mathrm{~m}, 2 \mathrm{H}), 1.37-1.29(\mathrm{~m}, 10 \mathrm{H}), 0.89(\mathrm{~m}$, $9 \mathrm{H}) .{ }^{13} \mathrm{C} \mathrm{NMR}\left(125 \mathrm{MHz}, \mathrm{CDCl}_{3}\right) \delta 79.7,72.5,38.4,31.8,29.6,29.3,26.7,22.7,22.5,18.8,14.1$. IR (neat) 3337, 2955, 2923, 2855, 1466, 1380, 1325, 1121, 1072, 1039, 965. HRMS (ESI) calcd. for $\mathrm{C}_{12} \mathrm{H}_{26} \mathrm{NaO}_{2}\left(\mathrm{M}+\mathrm{Na}^{+}\right)$225.1831, found 225.1834.

Representative procedure for Swern oxidation of alcohols: To a solution of oxalyl chloride (1.2 eq.) in $\mathrm{CH}_{2} \mathrm{Cl}_{2}$ (3 $\mathrm{mL}$ per mmol of ketone) was added DMSO (2 eq.) dropwise at $-78{ }^{\circ} \mathrm{C}$ and the reaction mixture was stirred for $30 \mathrm{~min}$ before the addition of alcohol (1 eq.). The resulting solution was stirred for a further 30 min then $\mathrm{Et}_{3} \mathrm{~N}$ (4 eq.) was added dropwise. After 
stirring for a further $1 \mathrm{~h} 15 \mathrm{~min}$ at $-78{ }^{\circ} \mathrm{C}$, the reaction was quenched with sat. aq. $\mathrm{NaHCO}_{3}$ (30 $\mathrm{mL}$ per mmol of ketone) and warmed to RT. The solution was extracted with $\mathrm{CH}_{2} \mathrm{Cl}_{2}(3 \mathrm{x}$ $30 \mathrm{~mL}$ per mmol of ketone) and then the organic fractions were combined and washed with sat. aq. $\mathrm{NaHSO}_{3}$ (30 mL per mmol of ketone), sat. brine (20 mL per mmol of ketone), then dried with $\mathrm{MgSO}_{4}$. The solvent was removed under reduced pressure and flash chromatography was used to purify the product.

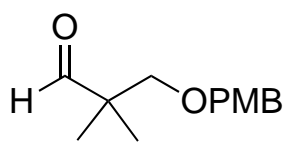

2,2-Dimethyl-3-benzyloxypropanal (27f): A Swern oxidation of 23 was carried out. The crude oil (50 mg, $0.22 \mathrm{mmol}$ ) was purified with flash chromatography (10:1 hexane:EtOAc) to provide a colorless oil $\mathbf{2 7 f}$ (26 $\mathrm{mg}$, $58 \%) .{ }^{1} \mathrm{H} \operatorname{NMR}\left(500 \mathrm{MHz}, \mathrm{CDCl}_{3}\right) \delta 9.55(\mathrm{~s}, 1 \mathrm{H}), 7.23(\mathrm{~d}, J=8.23 \mathrm{~Hz}, 2 \mathrm{H}), 6.88(\mathrm{~d}, J=$ $7.6 \mathrm{~Hz}, 2 \mathrm{H}), 4.44(\mathrm{~s}, 2 \mathrm{H}), 3.81(\mathrm{~s}, 3 \mathrm{H}), 3.42(\mathrm{~s}, 2 \mathrm{H}), 1.08(\mathrm{~s}, 6 \mathrm{H})$. Spectral data matched those reported by Turner. ${ }^{2}$<smiles>CC(=O)C(C)(C)C=O</smiles>

2,2-Dimethyl-3-oxy-decanal (27h): A modified Swern oxidation of $\mathbf{5 8}$ (40 mg, $0.2 \mathrm{mmol}$ ) was carried out with 3 eq. oxalyl chloride, 4 eq. DMSO and 8 eq. $\mathrm{Et}_{3} \mathrm{~N}$ and the rest of procedure was unchanged. The resulting crude oil was purified with flash chromatography (20:1 hexane:EtOAc) to provide $\mathbf{2 7 h}(7 \mathrm{mg}, 18 \%)$ which was volatile under high vacuum (0-1 mbar). ${ }^{1} \mathrm{H} \mathrm{NMR}\left(300 \mathrm{MHz}, \mathrm{CDCl}_{3}\right) \delta 9.62(\mathrm{~s}, 1 \mathrm{H})$, $2.45(\mathrm{t}, J=7.2 \mathrm{~Hz}, 2 \mathrm{H}), 1.58-1.54(\mathrm{~m}, 4 \mathrm{H}), 1.33$ (s, 6H), 1.26 (app. br. s, $6 \mathrm{H}), 0.90-0.86(\mathrm{~m}, 3 \mathrm{H})$. 


\section{References}

1. West, L. M.; Nothcote, P. T.; Battershill, C. N. J. Org. Chem. 2000, 65, 445-449.

2. Turner, E. Design and Synthesis of Hybrid Peloruside A - Laulimalide Analogues; PhD Thesis, Victoria University of Wellington, 2007.

3. Evans, D. A.; Welch, D. S.; Speed, A. W. H.; Moniz, G. A.; Reichelt, A. et al. J. Am. Chem. Soc. 2009, 131, 3840-3841.

4. Stocker, B. L.; Teesdale-Spittle, P.; Hoberg, J. O. Eur. J. Org. Chem. 2004, 330-336.

5. Stocker, B. Studies Towards the Synthesis of Peloruside A; PhD Thesis, Victoria University of Wellington, 2004.

6. Paton, R. S.; Goodman, J. M. J. Org. Chem. 2008, 73, 1253-1263.

7. Abiko, A.; Inoue, T.; Masamune, S. J. Am. Chem. Soc. 2002, 124, 10759-10764.

8. Balunas, M.; Kinghorn, A. Life Sci. 2005, 78, 431-441.

9. Hertweck, C. Angew. Chem., Int. Ed. 2009, 48, 4688-4716.

10. Liao, X.; Wu, Y.; Branbander, D. Angew. Chem., Int. Ed. 2003, 42, 1648-1652.

11. Hood, K. A.; Backstrőm, B. T.; West, L. M.; Nothcote, P. T.; Berridge, M. V. et al. AntiCancer Drug Des. 2001, 16, 155-166.

12. Gaitanos, T. N.; Buey, R. M.; Diaz, F.; Nothcote, P. T.; Burridge, M. V. et al. Cancer Res. 2004, 64, 5063.

13. Geary, L. M.; Hultin, P. G. Tetrahedron: Asym. 2009, 20, 131-173.

14. Arya, P.; Qin, H. Tetrahedron 2000, 56, 917-947.

15. Evans, D. A.; Dart, M. J.; Duffty, J. L.; Yang, M. G. J. Am. Chem. Soc. 1996, 118, 43224343.

16. Evans, D. A.; Cee, V. J.; Siska, S. J. J. Am. Chem. Soc. 2006, 128, 9433-9441.

17. Goodman, J. M.; Paton, R. S. Chem. Comm. 2007, 21, 2124-2126. 
18. Cram, D. J.; Elhafez, F. A. A. J. Am. Chem. Soc. 1952, 74, 5828-5835.

19. Chérest, M.; Felkin, H.; Prudent, N. Tetrahedron lett. 1968, 9, 2199-2204.

20. Anh, N. T.; Eisenstein, O.; Lefour, J.-M.; Dâu, M.-E. T. H. J. Am. Chem. Soc. 1973, 95, $6146-6147$.

21. Cornforth, J. W.; Cornforth, R. H.; Mathew, K. K. J. Chem. Soc. 1959, 112-127.

22. Evans, D. A.; Siska, S. J.; Cee, V. J. Angew. Chem., Int. Ed. 2003, 42, 1761-1765.

23. Mengel, A.; Reiser, O. Chem. Rev. 1999, 99, 1191-1224.

24. Samuels, W. D.; Nelson, D. A.; Hallen, R. T. Tetrahedron Lett. 1986, 27, 3091 - 3094.

25. Taniguchi, M.; Fujii, H.; Oshima, K.; Utimoto, K. Tetrahedron 1993, 49, 11169 - 11182.

26. Shibata, I.; Yoshida, T.; Kawakami, T.; Baba, A.; Matsuda, H. J. Org. Chem. 1992, 57, 4049-4051.

27. Fujita, M.; Hiyama, T. J. Org. Chem. 1988, 53, 5415-5421.

28. Chen, X.; Hortelano, E. R.; Eliel, E. L.; Frye, S. V. J. Am. Chem. Soc. 1990, 112, 6130-6131.

29. Reetz, M.; Kesseler, K.; Jung, A. Tetrahedron Lett. 1984, 25, 729 - 732.

30. Evans, D. A.; Duffy, J. L.; Dart, M. J. Tetrahedron Lett. 1994, 35, 8537 - 8540.

31. Arikan, F.; Li, J.; Menche, D. Org. Lett. 2008, 10, 3521-3524.

32. Paterson, I.; Goodman, J. M.; Lister, M. A.; Schumann, R. C.; McClure, C. K. et al. Tetrahedron 1990, 46, 4663-4684.

33. Gennari, C.; Moresca, D.; Vulpetti, A.; Pain, G. Tetrahedron Lett. 1994, 35, 4623 - 4626.

34. Gennari, C.; Hewkin, C. T.; Molinari, F.; Bernardi, A.; Comotti, A. et al. J. Org. Chem. 1992, 57, 5173-5177.

35. Diaz, L. C.; Aguilar, A. M. Chem. Soc. Rev. 2008, 37, 451-469.

36. Blanchette, M. A.; Malamas, M. S.; Nantz, M. H.; Roberts, J. C.; Somfai, P. et al. J. Org. Chem. 1989, 54, 2817-2825.

37. Evans, D. A.; Cóté, B.; Coleman, P. J.; Connell, B. T. J. Am. Chem. Soc. 2003, 125, 10893-10898.

38. Evans, D. A.; Coleman, P. J.; Cóté, B. J. Org. Chem. 1997, 62, 788-789.

39. Paterson, I.; Gibson, K. R.; Oballa, R. M. Tetrahedron Lett. 1996, 37, 8585-8588.

40. Inoue, T.; Liu, J.-F.; Buske, D. C.; Abiko, A. J. Org. Chem. 2002, 67, 5250-5256. 
41. Abiko, A.; Inoue, T.; Furuno, H.; Schwalbe, H.; Fieres, C. et al. Journal of the American Chemical Society 2001, 123, 4605-4606.

42. Zhao, M.; King, A. O.; Larsen, R. D.; Verhoeven, T. R.; Reider, P. J. et al. Tetrahedron Lett. 1997, 38, $2641-2644$.

43. List, B.; Lerner, R. A.; Barbas III, C. F. J. Am. Chem. Soc 2000, 122, 2395-2396.

44. Tho, F. Towards the Synthesis of Hybrid Peloruside A and Laulimalide Analogues; MBmedSc Thesis, Victoria University of Wellington, 2010.

45. List, B.; Hoang, L.; Martin, H. J. Proc. Natl. Acad. Sci. U. S. A. 2004, 101, 5839-5842.

46. Dias, L. C.; de Marchi, A. A.; Ferreira, M. A. B.; Aguilar, A. M. J. Org. Chem. 2008, 73, 6299-6311.

47. Delgado, M.; Martin, J. D. J. Org. Chem. 1999, 64, 4798-4816.

48. Casey, E. M.; Teesdale-Spittle, P.; Harvey, J. E. Tetrahedron Lett. 2008, 49, 7021-7023.

49. Cho, J. H.; Kim, B. M. Org. Lett. 2003, 5, 531-533.

50. Maynard, H. D.; Grubbs, R. H. Tetrahedron Lett. 1999, 40, $4137-4140$.

51. Paquette, L. A.; Schloss, J. D.; Efremov, I.; Fabris, F.; Gallou, F. et al. Org. Lett. 2000, 2, $1259-1261$.

52. Ahn, Y. M.; Yang, K.; Georg, G. I. Org. Lett. 2001, 3, 1411-1413.

53. Kobayashi, Y.; Tan, C.-H.; Kishi, Y. Helv. Chem. Acta 2000, 83, 2562-2571.

54. Evans, D. A.; Chapman, K. T.; Carreira, E. M. J. Am. Chem. Soc. 1988, 110, 3560-3578.

55. Schetter, B.; Mahrwald, R. Angew. Chem., Int. Ed. 2006, 45, 7506-7525.

56. Ghosh, A. K.; Fidanze, S.; Onishi, M.; Hussain, K. A. Tetrahedron Lett. 1997, 38, 7171 7174 .

57. Matsui, R.; Seto, K.; Fujita, K.; Suzuki, T.; Nakazaki, A. et al. Angew. Chem., Int. Ed. 2010, 49, 10068-10073.

58. Silverstein, R. M.; Webster, F. X.; Kiemle, D. J. Spectrometric Identification of Organic Compounds, 7th ed.; Wiley, 2005.

59. Kuroda, K.; Hayashi, Y.; Mukaiyama, T. Tetrahedron 2007, 63, 6358-6364.

60. Mitchell, T. A.; Bode, J. W. J. Am. Chem. Soc. 2009, 131, 18057-18059.

61. Corey, E. J.; Ensley, H. E. J. Am. Chem. Soc. 1975, 97, 6908-6909.

62. Mezrhab, B.; Dumas, F.; d'Angelo, J.; Riche, C. J. Org. Chem. 1994, 59, 500-503. 
63. Capim, S. L.; Santana, S. R.; Oliveira, B. G. d.; Rocha, G. B.; Vasconcellos, M. J. Braz. Chem. Soc. 2010, 21, $1718-1726$.

64. Hoye, T. R.; Jeon, J.; Kopel, L. C.; Ryba, T. D.; Tennakoon, M. A. et al. Angew. Chem., Int. Ed. 2010, 49, 6151-6155.

65. Lyer, K.; Rainier, J. D. J. Am. Chem. Soc. 2007, 129, 12604-12605.

66. Fujiwara, T.; Kato, Y.; Takeda, T. Tetrahedron Lett. 2000, 56, 4859-4869.

67. Mukaiyama, T.; Stevens, R. W.; lwasawa, N. Chem. Lett. 1982, 11, 353-356.

68. Evans, D. A.; Allison, B. D.; Yang, M. G. Tetrahedron Lett. 1999, 40, 4457-4460.

69. Ertas, M.; Seebach, D. Helv. Chim. Acta 1985, 68, 961-968. 